Supplementary Information for:

\title{
Mechanism and Scope of Nickel-Catalyzed Decarbonylative Borylation of Carboxylic Acid Fluorides
}

\author{
Christian A. Malapit, James R. Bour, Simon R. Laursen, and Melanie S. Sanford* \\ Department of Chemistry, University of Michigan \\ 930 North University Avenue, Ann Arbor, Ml 48109 United States \\ *Email: mssanfor@umich.edu
}

\section{Table of Contents}

I. General information $\quad$ S2

II. Materials and methods $\quad$ S2

III. Stoichiometric studies on oxidative addition and decarbonylation

of aroyl fluorides $\quad$ S3

IV. Synthesis of aryl nickel halide complexes

V. Transmetalation and reductive elimination studies of complex B with aryl boronic $\quad$ S7 acids and esters

VI. Transmetalation and reductive elimination studies of complex B with diboron S8 reagents

VII. Studies on attempts to observe nickel boronate intermediates $\mathbf{C} \quad \mathrm{S} 10$

VIII. Reaction of complexes B2 and B3 with 4-fluorophenyl boronic acid 1 and $\mathrm{B}_{2}(\mathrm{neo})_{2} \quad \mathrm{~S} 12$

IX. Optimization of Ni-catalyzed decarbonylative borylation S13

X. Catalytic reactions at low temperatures and resting state analysis $\quad \mathrm{S} 18$

XI. Ni-catalyzed decarbonylative borylation via in situ formation of acid fluoride $\quad$ S20

XII. Selectivity studies $\quad$ S21

XIII. Synthesis of carboxylic acid fluorides $\quad$ S23

XIV. Scope of Ni-catalyzed decarbonylative borylation S26

XV. References $\mathrm{S} 43$

XVI. Copies of ${ }^{1} \mathrm{H},{ }^{13} \mathrm{C},{ }^{19} \mathrm{~F}$ and ${ }^{31} \mathrm{P}$ NMR Spectra S44-146 


\section{General information}

All NMR experiments were recorded using Varian MR400 (400.52 $\mathrm{MHz}$ for ${ }^{1} \mathrm{H}, 100.71 \mathrm{MHz}$ for ${ }^{13} \mathrm{C}, 376.87 \mathrm{MHz}$ for $\left.{ }^{19} \mathrm{~F}\right)$, Varian vnmrs $500\left(500.01 \mathrm{MHz}\right.$ for ${ }^{1} \mathrm{H}, 125.75 \mathrm{MHz}$ for ${ }^{13} \mathrm{C}, 470.56 \mathrm{MHz}$ for $19 \mathrm{~F}$ ), or Varian nmrs $700\left(699.76 \mathrm{MHz}\right.$ for ${ }^{1} \mathrm{H}, 175.95 \mathrm{MHz}$ for $\left.{ }^{13} \mathrm{C}\right)$ spectrometers. Chemical shifts $(\delta)$ are reported in ppm, and coupling constants $(J)$ are reported in $\mathrm{Hz}$. The 7.26 resonance of residual $\mathrm{CHCl}_{3}$ for proton spectra and the $77.23 \mathrm{ppm}$ resonance of $\mathrm{CDCl}_{3}$ for carbon spectra were used as internal references. High-resolution mass spectrometry data (HRMS) were obtained on a Micromass AutoSpec Ultima Magnetic Sector instrument. GCMS analyses were performed on a Shimadzu GCMS-QP2010 gas chromatograph mass spectrometer. Melting points were determined with a Mel-Temp 3.0 (Laboratory Devices, Inc.) and are uncorrected. Chromatographic purifications were performed using 40-63 micron flash silica gel or a CombiFlash Torrent $^{\circledR}$ system using RediSep ${ }^{\circledR} \mathrm{Rf}$ columns packed with silica gel. X-ray crystallographic data were obtained on a Rigaku AFC10K Saturn 944+ CCD-based X-ray diffractometer.

\section{Materials and methods}

All commercially available reagents were used as received unless otherwise stated. $\mathrm{Ni}(\mathrm{cod})_{2}$ and $\mathrm{PC}_{3}$ were purchased from Sigma Aldrich and stored in a glovebox. Benzoyl fluoride 1 (SynQuest) and 4-trifluoromethylbenzoyl fluoride 15-COF (SynQuest) were purchased from commercial sources and used as received. Tetramethylfluoroformamidiniun hexafluorophosphate (TFFH, Chem Impex), proton sponge (Sigma), CsF (Alfa Aesar), and (o-tolyl)( $\left.\mathrm{PCy}{ }_{3}\right)_{2} \mathrm{Ni}^{\prime \prime}(\mathrm{Cl})$ (Sigma) were purchased from commercial sources and stored under an inert atmosphere. Diboron reagents, and aryl boronic acids and esters, were purchased from commercial sources (Sigma, Alfa Aesar, Matrix Scientific, Frontier Scientific, Synquest) and used as received. Deuterated solvents were purchased from Cambridge Isotope Laboratories, Inc. 


\section{Stoichiometric studies on oxidative addition and decarbonylation of aroyl fluorides}

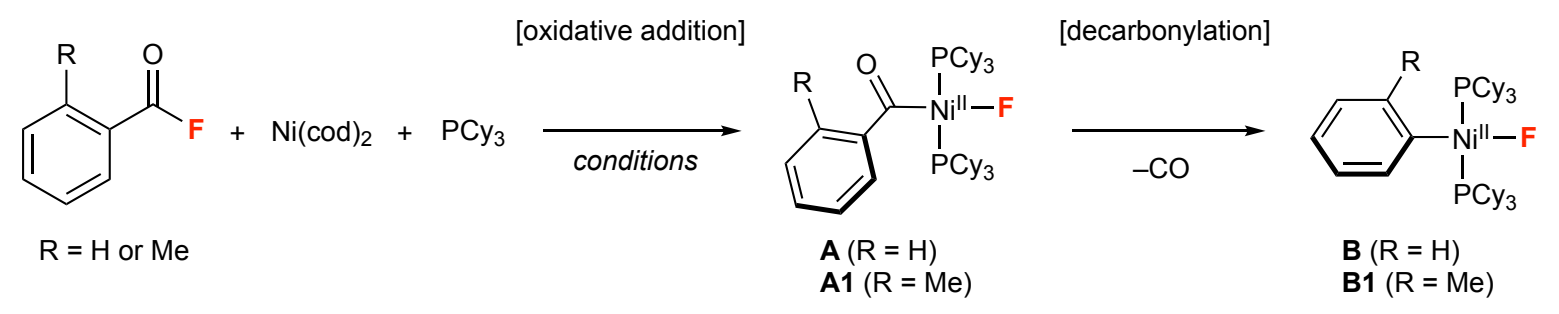

\begin{tabular}{|c|c|c|c|}
\hline Substrate & conditions & $t_{1 / 2} \mathbf{A}$ or $\mathbf{A} \mathbf{1}$ & $t_{1 / 2} \mathbf{B}$ or $\mathbf{B} 1$ \\
\hline & THF, rt & $<5 \min$ & $170 \min$ \\
\hline & $\mathrm{THF}, 45^{\circ} \mathrm{C}$ & $<5 \min$ & $30 \mathrm{~min}$ \\
\hline & toluene, $45^{\circ} \mathrm{C}$ & $<5 \min$ & $24 \min$ \\
\hline & THF, rt & $<5 \min$ & $90 \mathrm{~min}$ \\
\hline
\end{tabular}

Table S1. Stoichiometric studies on the oxidative addition and decarbonylation of acid fluorides.

In a glovebox, a solution of $\mathrm{Ni}(\mathrm{cod})_{2}\left(0.04 \mathrm{mmol}, 2.0\right.$ equiv) and $\mathrm{PCy}_{3}(0.08 \mathrm{mmol}, 4.0$ equiv) in THF $(0.25 \mathrm{~mL}$, from a stock solution) was stirred in a $4 \mathrm{~mL}$ vial at room temperature for $10 \mathrm{~min}$. In a separate $4 \mathrm{~mL}$ vial, a solution of benzoyl fluoride $(0.02 \mathrm{mmol}, 1.0$ equiv) and 2fluoromesitylene $(0.02 \mathrm{mmol}, 1.0$ equiv, internal standard $)$ in THF $(0.25 \mathrm{~mL}$, from a stock solution) was stirred for $5 \mathrm{~min}$. Both vials were cooled to $-35^{\circ} \mathrm{C}$ by placing inside the glovebox freezer for 20-30 min. The vials were removed from the freezer, the solutions were combined, and then the resulting solution was transferred to a J. Young NMR tube. The tube was sealed and removed from the glove box. Starting at $5 \mathrm{~min}$ from the time of mixing, the reaction mixture was analyzed by ${ }^{19} \mathrm{~F}$ NMR spectroscopy.

The starting benzoyl fluoride 1 was completely consumed within $10 \mathrm{~min}$, with concomitant formation of the oxidative addition product $(\mathrm{PhCO}) \mathrm{Ni}(\mathrm{F})\left(\mathrm{PC}_{3}\right)_{2} 2\left(>95 \%{ }^{19} \mathrm{~F}\right.$ NMR yield, $\left.10 \mathrm{~min}\right)$ : ${ }^{19} \mathrm{~F}$ NMR $-327.9(\mathrm{t}, J=46 \mathrm{~Hz})$. Complex 2 then underwent decarbonylation to form $(\mathrm{Ph}) \mathrm{Ni}(\mathrm{F})(\mathrm{PCy})_{2} 3\left(50 \%{ }^{19} \mathrm{~F}\right.$ NMR yield after $\left.170 \mathrm{~min}\right):{ }^{19} \mathrm{~F}$ NMR $-363.7(\mathrm{t}, J=42 \mathrm{~Hz})$. During these stoichiometric studies, $\mathrm{Ni}(\mathrm{CO})_{2}\left(\mathrm{PCy}_{3}\right)_{2}$ was observed as a byproduct, as confirmed by ${ }^{31} \mathrm{P}$ $\operatorname{NMR}(40.6 \mathrm{ppm})$ and FTIR analysis (CO stretches at 1980 and $\left.1915 \mathrm{~cm}^{-1}\right)^{1}$

Under similar conditions, the reactions were performed at $45^{\circ} \mathrm{C}$, toluene as solvent and o-toloyl fluoride as the substrate. The results are summarized in Table $\mathrm{S} 1$. 


\section{Synthesis of aryl nickel halide complexes}
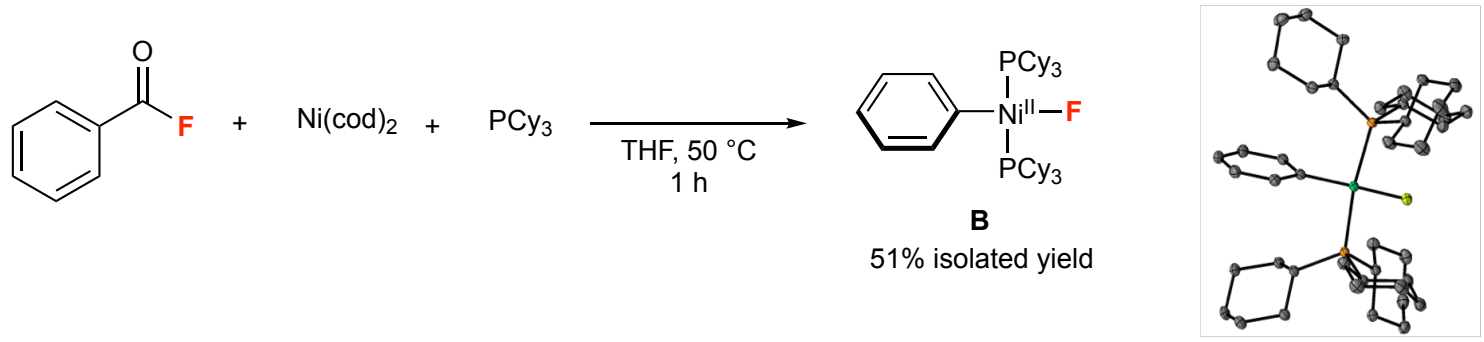

X-ray structure of $\mathbf{B}^{1}$

Synthesis of $\mathbf{B}\left[\left(\mathbf{P C y}_{3}\right)_{2} \mathbf{N i}(\mathbf{F})(\mathbf{P h})\right]$. Complex B was prepared according to our previous report. ${ }^{2}$ In a glovebox, a $100 \mathrm{~mL}$ flask was charged with $\mathrm{Ni}(\mathrm{cod})_{2}\left(550 \mathrm{mg}, 2.0 \mathrm{mmol}, 1\right.$ equiv), $\mathrm{PCy}_{3}(1.20$ g, $4.3 \mathrm{mmol}, 2.15$ equiv), THF (30 mL), and a magnetic stir bar. The solution was stirred at room temperature for $25 \mathrm{~min}$ until all of the $\mathrm{Ni}(\mathrm{cod})_{2}$ dissolved. A rubber septum was added to the flask, and the solution was placed in a $-35^{\circ} \mathrm{C}$ freezer for $20 \mathrm{~min}$. While the Ni solution cooled, a $4 \mathrm{~mL}$ vial was charged with benzoyl fluoride (298 mg, $2.4 \mathrm{mmol}, 1.20$ equiv) and THF (1 mL). This solution was added in one portion to the rapidly stirring solution of $\mathrm{Ni}(\operatorname{cod})_{2} / \mathrm{PC} y_{3}$. Upon mixing, the solution rapidly changed from a dark orange to a bright orange. After stirring for $5 \mathrm{~min}$, an additional $30 \mathrm{~mL}$ of THF was added. The flask was then sealed with a rubber septum, removed from the glovebox, and placed in a preheated $\left(50^{\circ} \mathrm{C}\right)$ oil bath. While still in the oil bath, the solution was sparged with a gentle stream of $\mathrm{N}_{2}$ for $1 \mathrm{~h}$. The flask was removed from the oil bath, allowed to cool to room temperature, and brought back into the glovebox. The volatiles were removed under reduced pressure until the volume was approximately $15 \mathrm{~mL}$, at which point $\mathrm{Et} \mathrm{t}_{2} \mathrm{O}(30 \mathrm{~mL})$ was added to precipitate a yellow solid. The yellow precipitate was collected on a frit, washed with $\mathrm{Et}_{2} \mathrm{O}(2 \times 10 \mathrm{~mL})$ and pentanes $(10 \mathrm{~mL})$, and dried under vacuum to yield $\mathbf{B}$ as a bright yellow powder (730 mg, 51\% yield). ${ }^{1} \mathrm{H}$ NMR $\left(700 \mathrm{MHz}, \mathrm{C}_{6} \mathrm{D}_{6}\right) \delta 7.72(\mathrm{~d}, J=7.3 \mathrm{~Hz}, 2 \mathrm{H}), 6.94(\mathrm{t}, J=7.3$ $\mathrm{Hz}, 2 \mathrm{H}$ ), $6.78(\mathrm{t}, J=7.3 \mathrm{~Hz}, 1 \mathrm{H}), 2.21-2.09$ (br, 12H), 1.89-1.60 (multiple peaks, 36H), 1.29-1.09 (multiple peaks, $18 \mathrm{H}) ;{ }^{13} \mathrm{C}$ NMR $\left(176 \mathrm{MHz}, \mathrm{C}_{6} \mathrm{D}_{6}\right) \delta 150.22(\mathrm{~m}), 140.52,125.41,120.74,32.98(\mathrm{t}$, $J=8.2 \mathrm{~Hz}), 30.44,28.49(\mathrm{t}, J=5.1 \mathrm{~Hz}), 27.40 ;{ }^{19} \mathrm{~F}$ NMR $\left(470 \mathrm{MHz}, \mathrm{C}_{6} \mathrm{D}_{6}\right) \delta-363.2(\mathrm{t}, J=42.7$ $\mathrm{Hz}) ;{ }^{31} \mathrm{P}$ NMR $\left(283 \mathrm{MHz}, \mathrm{C}_{6} \mathrm{D}_{6}\right) \delta 15.77(\mathrm{~d}, \mathrm{~J}=42.0 \mathrm{~Hz}$ ). Complex B was stored under air and found stable for 7 days. No significant changes or decomposition was observed by ${ }^{19} \mathrm{~F}$ and ${ }^{31} \mathrm{P}$ NMR spectroscopy.

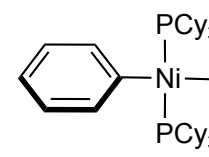

B

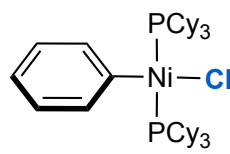

B2

Synthesis of $\mathbf{B 2}\left[\left(\mathrm{PCy}_{3}\right)_{2} \mathbf{N i}(\mathbf{C l})(\mathbf{P h})\right.$. $^{2}$ In a glovebox, a $20 \mathrm{~mL}$ vial was charged with $\mathbf{B}$ (75 mg, $0.10 \mathrm{mmol}, 1$ equiv) and THF (2 mL). To this solution was added TMSCl as a freshly prepared 
stock solution (0.1 M in THF, $1.25 \mathrm{~mL}, 1.2$ equiv). The vial was capped, gently shaken, and allowed to stand at room temperature. Over the course of $2 \mathrm{~h}$ the solution changed color from bright yellow to orange. After $2 \mathrm{~h}$, the volatiles were removed under reduced pressure, and the resulting yellow solid was triturated with $\mathrm{Et}_{2} \mathrm{O}(3 \mathrm{~mL})$ and then dried under vacuum. Complex $\mathbf{B 2}$ was obtained as a yellow powder $(55 \mathrm{mg}, 72 \%$ yield $) .{ }^{2}{ }^{1} \mathrm{H} \mathrm{NMR}\left(700 \mathrm{MHz}, \mathrm{C}_{6} \mathrm{D}_{6}\right) \delta 7.74(\mathrm{~d}, \mathrm{~J}=$ $7.4 \mathrm{~Hz}, 2 \mathrm{H}$ ), $6.92(\mathrm{t}, J=7.4 \mathrm{~Hz}, 2 \mathrm{H}), 6.78(\mathrm{t}, J=7.3 \mathrm{~Hz}, 1 \mathrm{H}$ ), 2.16-2.02 (multiple peaks, $18 \mathrm{H}$ ), 1.87-1.63 (multiple peaks, 30H), 1.25-1.11 (multiple peaks, $18 \mathrm{H}) ;{ }^{13} \mathrm{C}$ NMR $\left(176 \mathrm{MHz}, \mathrm{C}_{6} \mathrm{D}_{6}\right) \delta$ 150.09 (t, $J=32.6 \mathrm{~Hz}), 139.71$ (t, $J=3.1 \mathrm{~Hz}$ ), 125.30 (t, $J=2.4 \mathrm{~Hz}), 120.70$ (t, $J=2.3 \mathrm{~Hz}$ ), 33.77 $(\mathrm{t}, J=8.6 \mathrm{~Hz}), 29.99,27.81(\mathrm{t}, J=4.8 \mathrm{~Hz}), 26.65 ;{ }^{31} \mathrm{P}$ NMR $\left(283 \mathrm{MHz}, \mathrm{C}_{6} \mathrm{D}_{6}\right) \delta 12.20$; Elemental analysis: calculated for $\mathrm{C}_{42} \mathrm{H}_{71} \mathrm{P}_{2} \mathrm{CINi}, \mathrm{C}$ : $68.90 \mathrm{H}$ : 9.78 ; found $\mathrm{C}: 68.77 \mathrm{H}: 9.68$.

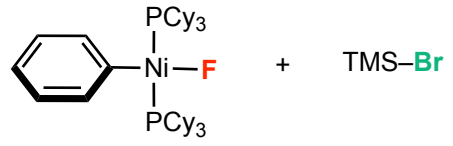

B

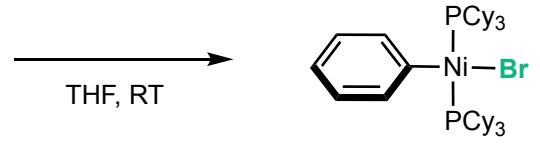

B3

Synthesis of $\mathbf{B} 3\left[\left(\mathrm{PC}_{3}\right)_{2} \mathbf{N i}(\mathrm{Br})(\mathbf{P h})\right]^{2}$ In a glovebox, a $20 \mathrm{~mL}$ vial was charged with $\mathbf{B}$ (75 mg, $0.10 \mathrm{mmol}, 1$ equiv) and THF (2 mL). To this solution was added $\mathrm{TMSBr}$ as a freshly prepared stock solution $(0.1 \mathrm{M}$ in THF, $1.25 \mathrm{~mL}, 1.2$ equiv). The vial was capped, gently shaken, and allowed to stand at room temperature. Over the course of $10 \mathrm{~min}$ the solution changed color from bright yellow to a dark orange. After $10 \mathrm{~min}$, the volatiles were removed under reduced pressure, and the resulting yellow solid was triturated with $\mathrm{Et}_{2} \mathrm{O}(3 \mathrm{~mL})$ and dried under vacuum. Complex B3 was obtained as a yellow powder (52 mg, 65\% yield). ${ }^{2} \mathrm{H}$ NMR (700 MHz, $\left.\mathrm{C}_{6} \mathrm{D}_{6}\right) \delta 7.74$ (d, J $=7.4 \mathrm{~Hz}, 2 \mathrm{H}), 6.92(\mathrm{t}, J=7.4 \mathrm{~Hz}, 2 \mathrm{H}), 6.76(\mathrm{t}, J=7.3 \mathrm{~Hz}, 1 \mathrm{H}), 2.17-2.12$ (multiple peaks, $18 \mathrm{H}$ ), 1.82-1.61 (multiple peaks, 30H), 1.23-1.10 (multiple peaks, $18 \mathrm{H}) ;{ }^{13} \mathrm{C}$ NMR $\left(176 \mathrm{MHz}, \mathrm{C}_{6} \mathrm{D}_{6}\right) \delta$ $150.71(\mathrm{t}, J=34.8 \mathrm{~Hz}), 139.60,125.30(\mathrm{~d}, J=2.8 \mathrm{~Hz}), 120.92,34.48(\mathrm{t}, J=8.6 \mathrm{~Hz}), 30.13,27.80$ $(\mathrm{t}, J=4.8 \mathrm{~Hz}), 26.61 ;{ }^{31} \mathrm{P}$ NMR $\left(202 \mathrm{MHz}, \mathrm{C}_{6} \mathrm{D}_{6}\right) \delta 11.42$. The isolated complex contains traces of $\mathrm{O}=\mathrm{PCy}_{3}$ based on the ${ }^{31} \mathrm{P}$ NMR spectrum. Elemental analysis: calculated for $\mathrm{C}_{42} \mathrm{H}_{71} \mathrm{P}_{2} \mathrm{BrNi}, \mathrm{C}$ : $64.96 \mathrm{H}: 9.22$; found $\mathrm{C}: 64.68 \mathrm{H}: 9.45$.

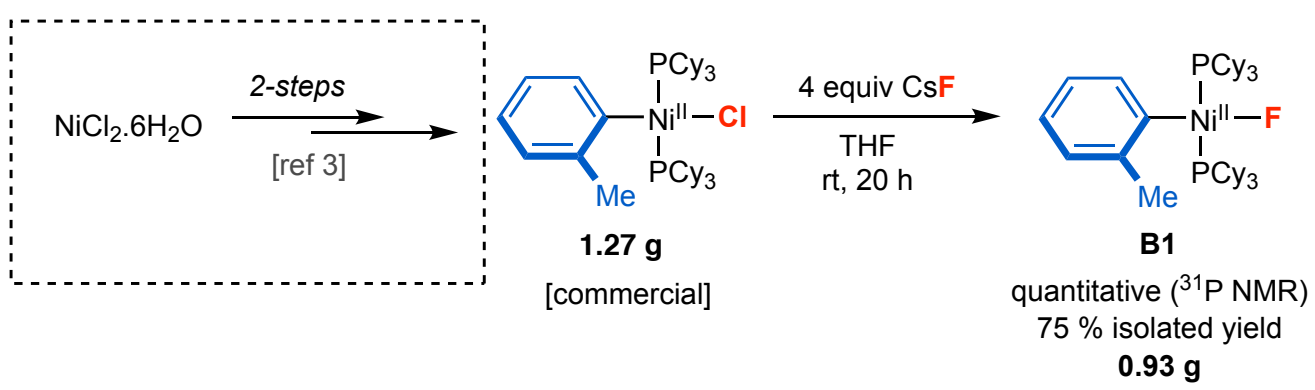

Gram-Scale Synthesis of B1 [(PCy $)_{2} \mathbf{N i}(\mathbf{F})($ o-tol)]. In a glovebox, a $100 \mathrm{~mL}$ flask was charged with $\left[\left(\mathrm{PCy}_{3}\right)_{2} \mathrm{Ni}(\mathrm{Cl})(\mathrm{o} \text {-tol })\right]^{3}(1.27 \mathrm{~g}, 1.7 \mathrm{mmol}, 1$ equiv), $\mathrm{CsF}(1.03 \mathrm{~g}, 6.8 \mathrm{mmol}, 4$ equiv), THF (40 $\mathrm{mL}$ ), and a magnetic stir bar. We note that the $\mathrm{Ni-Cl}$ complex is also commercially available (CAS: 
123839-28-1). The resulting suspension was stirred vigorously at room temperature for $20 \mathrm{~h}$ or until all of the $\left[\left(\mathrm{PC}_{3}\right)_{2} \mathrm{Ni}(\mathrm{Cl})(\mathrm{o}-\mathrm{tol})\right]$ was consumed as monitored by ${ }^{31} \mathrm{P}$ NMR spectroscopy. (Note: The use of TMAF as fluoride source (in place of CsF) was also found to be effective but required longer reaction time to obtain full conversion. The use of KF did not provide observable conversion after $24 \mathrm{~h}$ ). Stirring was stopped, which allowed the insoluble salts ( $\mathrm{CsF} / \mathrm{CsCl})$ to settle at the bottom of the flask. The solution containing the Ni complex was decanted away from these salts and filtered through filter paper. The salt residue was washed with THF $(2 \times 10 \mathrm{~mL})$, and these washes were filtered through filter paper. The filtrate was concentrated under vacuum, and the resulting solid was dissolved in benzene. This solution was filtered through a plug of cotton. The filtrate was again concentrated under vacuum to provide B1 as a bright yellow powder: $(0.93 \mathrm{~g}$, $75 \%$ yield). ${ }^{1} \mathrm{H}$ NMR $\left(700 \mathrm{MHz}, \mathrm{C}_{6} \mathrm{D}_{6}\right) \delta 7.84$ (d, $J=7.3 \mathrm{~Hz}, 1 \mathrm{H}$ ), 6.82 (multiple peaks, $3 \mathrm{H}$ ), 3.40 (s, 3H), $2.31(\mathrm{~m}, 6 \mathrm{H}), 2.07(\mathrm{~m}, 6 \mathrm{H}), 1.94-1.64$ (multiple peaks, 34H), 1.40-1.10 (multiple peaks, 20H); ${ }^{13} \mathrm{C}$ NMR $\left(176 \mathrm{MHz}, \mathrm{C}_{6} \mathrm{D}_{6}\right) \delta 146.60(\mathrm{~m}), 143.52,139.91,126.03,122.07,121.06,33.02(\mathrm{t}$, $J=7.9 \mathrm{~Hz}), 30.29,29.27,27.88(\mathrm{t}, J=4.8 \mathrm{~Hz}), 26.60 ;{ }^{19} \mathrm{~F} \mathrm{NMR}\left(470 \mathrm{MHz}, \mathrm{C}_{6} \mathrm{D}_{6}\right) \delta-367.6(\mathrm{t}, J=$ $42.5 \mathrm{~Hz}) ;{ }^{31} \mathrm{P}$ NMR $\left(202 \mathrm{MHz}, \mathrm{C}_{6} \mathrm{D}_{6}\right) \delta 14.12(\mathrm{~d}, \mathrm{~J}=44.0 \mathrm{~Hz})$. Complex B1 was stored under air and found to be stable for 7 days. No significant changes or decomposition was observed by ${ }^{19} \mathrm{~F}$ and ${ }^{31} \mathrm{P}$ NMR spectroscopy over this time. 


\section{Transmetalation and reductive elimination studies of complex B with aryl boronic acids and boronate esters}

In a glovebox, $(\mathrm{Ph}) \mathrm{Ni}\left(\mathrm{PCy}_{3}\right)_{2}(\mathrm{~F}) \mathrm{B}(0.01 \mathrm{mmol}, 1.0$ equiv) was transferred into a $4 \mathrm{~mL}$ vial from a stock solution in toluene. 4-Fluorophenylboronic acid (0.012 mmol, 1.2 equiv) and 2fluoromesitylene $(0.01 \mathrm{mmol}, 1.0$ equiv) were added from stock solutions in toluene resulting in a total volume of $0.6 \mathrm{~mL}$. The reaction mixture was stirred at room temperature for $15-30 \mathrm{~s}$ and then immediately transferred to a J. Young NMR tube. The reaction was monitored by ${ }^{19} \mathrm{~F}$ NMR spectroscopy. A similar procedure was performed using various aryl boronate esters. Reactions of non fluorine-containing aryl boronate esters were performed in $4 \mathrm{~mL}$ vials, stirred at the appropriate temperature, and then analyzed by GC/GCMS after a given time. The results are summarized in Figure S1.

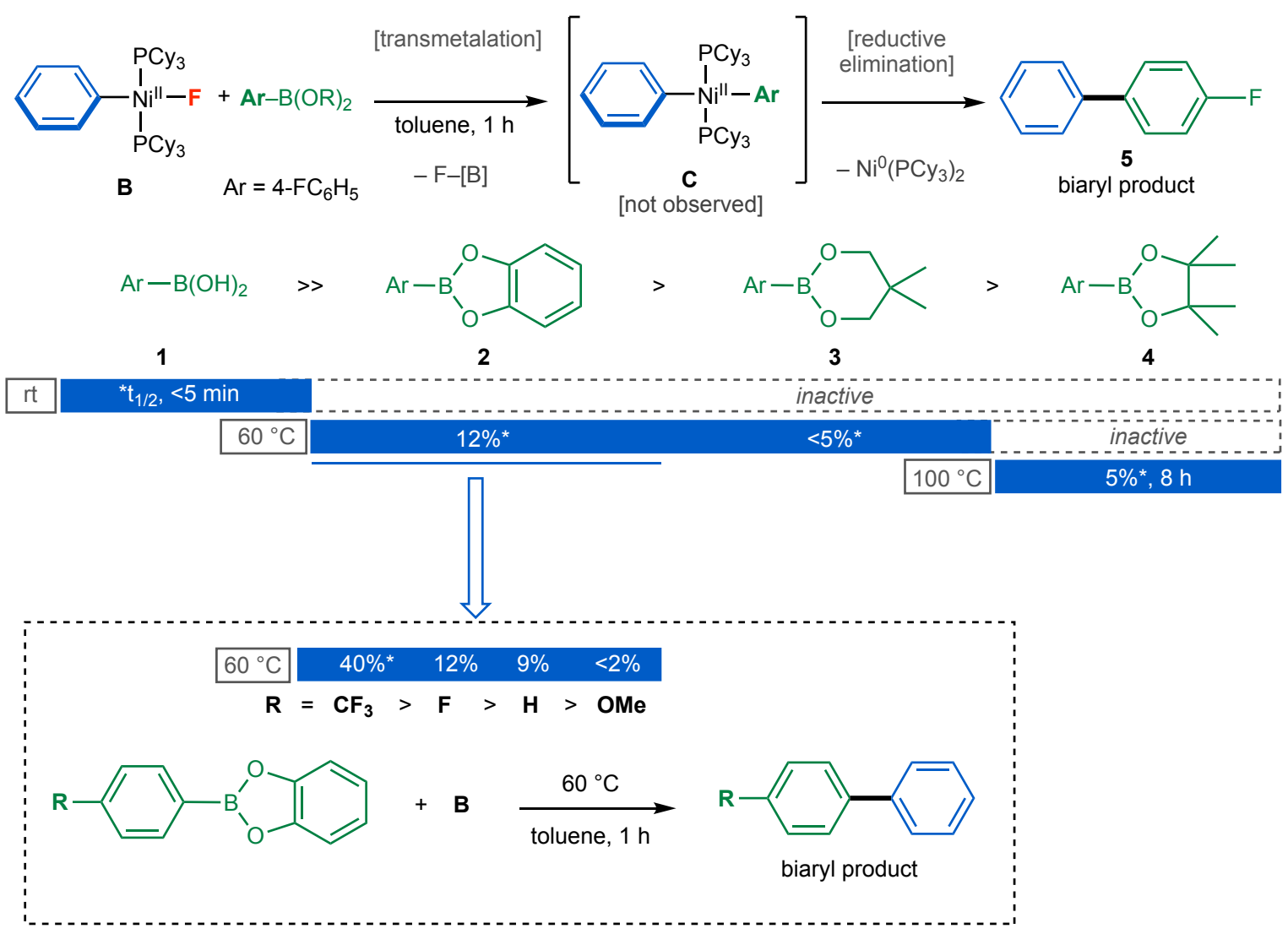

Figure S1. Transmetalation and reductive elimination of nickel complex $\mathbf{B}$ with aryl boronic acids and boronate esters 


\section{Transmetalation and reductive elimination studies of complex B with diboron reagents}

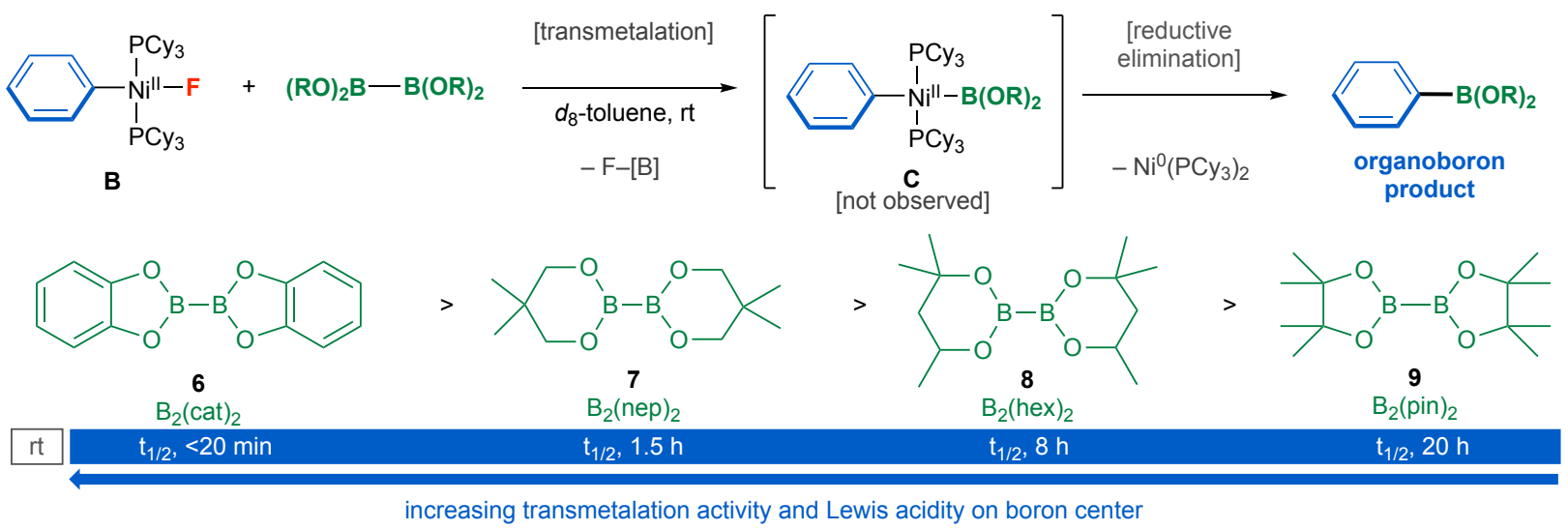

Figure S2. Reaction of nickel complex $\mathbf{B}$ with diboron reagents via transmetalation and reductive elimination.

In a glovebox, $(\mathrm{Ph}) \mathrm{Ni}\left(\mathrm{PCy}_{3}\right)_{2}(\mathrm{~F}) \mathbf{B}(0.01 \mathrm{mmol}, 1.0$ equiv $)$ was transferred into a $4 \mathrm{~mL}$ vial from a stock solution in $d_{8}$-toluene. $B_{2}$ (neo $)_{2} 7$ (0.012 mmol, 1.2 equiv) and 1,3,5-trimethoxybenzene ( $0.01 \mathrm{mmol}, 1.0$ equiv) were added from stock solutions in $d_{8}$-toluene resulting in a total volume of $0.6 \mathrm{~mL}$. The reaction mixture was stirred at room temperature for 15-30 s and immediately transferred to a J. Young NMR tube. The reaction was monitored by ${ }^{1} \mathrm{H}$ NMR spectroscopy. A similar procedure was performed using various diboron reagents $(\mathbf{6}, \mathbf{8}$, and 9 ) at room temperature and monitored by ${ }^{1} \mathrm{H}$ NMR spectroscopy. Results are summarized in Figures $\mathrm{S} 2$ and S3A and S3B. In addition, the reaction of complex B1 with $\mathrm{B}_{2}(\text { pin })_{2}(\mathbf{9})$ gave $11 \%$ conversion after $20 \mathrm{~h}$ at room temperature. The lower reactivity of complex B1 over B is likely due to steric hinderance from the ortho-methyl group, which slows transmetalation and reductive elimination.

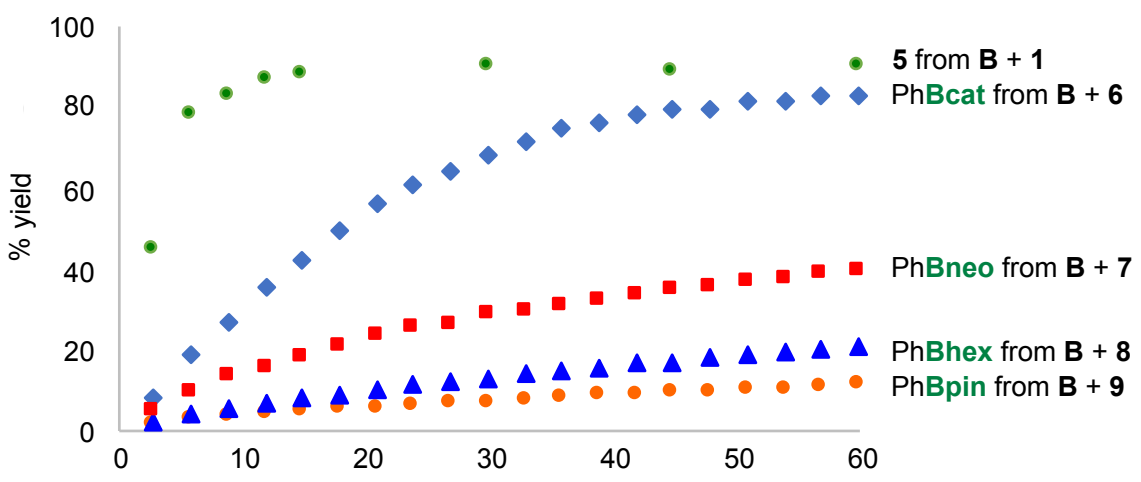

Figure S3A. Product formation from the transmetalation and reductive elimination of nickel complex B with 4-fluorophenyl boronic acid 1 and diboron reagents 6 to 9 . 


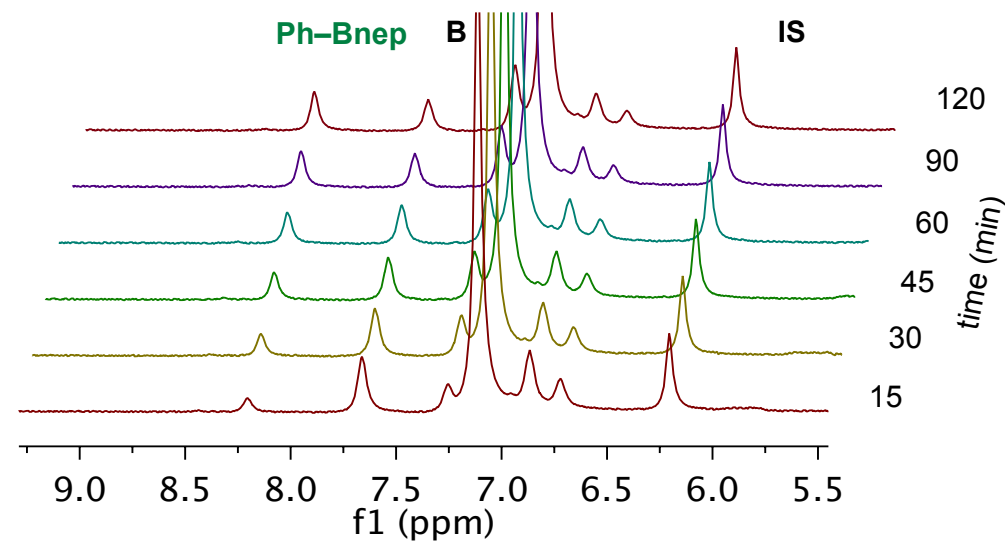

Figure S3B. Representative partial ${ }^{1} \mathrm{H}$ NMR spectra of the reaction of $\mathbf{B}$ and $\mathbf{7}$ to form $\mathrm{Ph}-\mathrm{Bnep}$. 
VII. Attempts at observing phenyl Ni-boronate intermediate C.

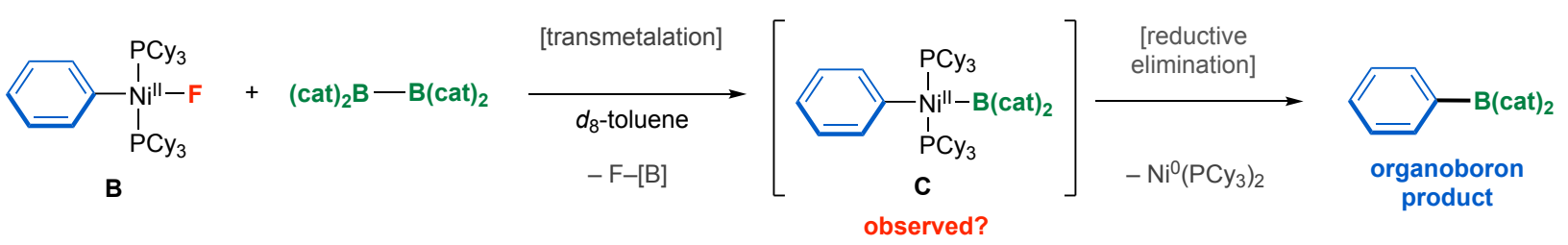

In a glovebox, a 2:1 admix of $(\mathrm{Ph}) \mathrm{Ni}\left(\mathrm{PC} y_{3}\right)_{2}(\mathrm{~F}) \mathrm{B}$ and $(\mathrm{PCy})_{2} \mathrm{Ni}(\mathrm{CO})_{2}$ (effective molecular weight: $705 \mathrm{~g} / \mathrm{mol}$ ) was transferred into a screw-capped NMR tube from a $d_{8}$-toluene stock solution resulting to $(\mathrm{Ph}) \mathrm{Ni}\left(\mathrm{PCy}_{3}\right)_{2}(\mathrm{~F}) \mathrm{B}\left(0.01 \mathrm{mmol}, 1.0\right.$ equiv) and $\left(\mathrm{PCy}_{3}\right)_{2} \mathrm{Ni}(\mathrm{CO})_{2}$ (as internal standard, $0.0033 \mathrm{mmol}, 0.33$ equiv). Additional $d_{8}$-toluene was added to the NMR tune to obtain $\sim 0.4 \mathrm{~mL}$ solution. In a 4-mL vial, $\mathrm{B}_{2}(\mathrm{cat}){ }_{2} 6(0.012 \mathrm{mmol}, 1.2$ equiv) and 4-fluorotoluene $(0.01 \mathrm{mmol}, 1.0$ equiv) were mixed from stock solutions in $d_{8}$-toluene resulting in a total volume of $0.2 \mathrm{~mL}$. Both solutions were taken out of the glovebox and cooled to $-75^{\circ} \mathrm{C}$. The solution containing $\mathrm{B}_{2}(\mathrm{cat})_{2} 6$ and 4-fluorotoluene was rapidly added to the solution in the NMR tube at $-75^{\circ} \mathrm{C}$. The reaction mixture was monitored by ${ }^{19} \mathrm{~F}$ and ${ }^{31} \mathrm{P}$ NMR spectroscopy at variable temperatures starting at $65^{\circ} \mathrm{C}$. A similar procedure was performed using various diboron reagents (7 and 9 ). Results are summarized in Figures S4A and S4B.

As seen in Figure S4A ( ${ }^{31} \mathrm{P}$ NMR), at $-65{ }^{\circ} \mathrm{C}$, formation of broad peak at $16 \mathrm{ppm}$ was observed. We believe that there are multiple overlapping peaks at this stage as indicated by ${ }^{19} \mathrm{~F}$ NMR (Fig. $\mathrm{S} 4 \mathrm{~B})$ at the same temperature and time. Allowing the reaction to proceed at $-65{ }^{\circ} \mathrm{C}$ over a prolonged period of time (up to $10 \mathrm{~h}$ ) did not lead to significant changes in the spectrum. Warming the reactions to $-35^{\circ} \mathrm{C}, 0{ }^{\circ} \mathrm{C}$ and $15^{\circ} \mathrm{C}$ did not show significant formation of a single or major peak on both ${ }^{31} \mathrm{P}$ and ${ }^{19} \mathrm{~F}$ NMR. At $15^{\circ} \mathrm{C}$, we note complete consumption of the starting Ni-F complex $\mathrm{B}$ based on ${ }^{31} \mathrm{P}$ and ${ }^{19} \mathrm{~F}$ NMR. Analysis of the reaction by $\mathrm{GC}$ at this time point showed $85 \%$ yield of PhBcat. Additionally, the use of $B_{2}(\text { nep })_{2}$ and $B_{2}(\text { pin })_{2}$ instead of $B_{2}$ (cat) $)_{2}$ led to no reactivity at low temperatures. Importantly, no intermediates were observed at room temperature. 


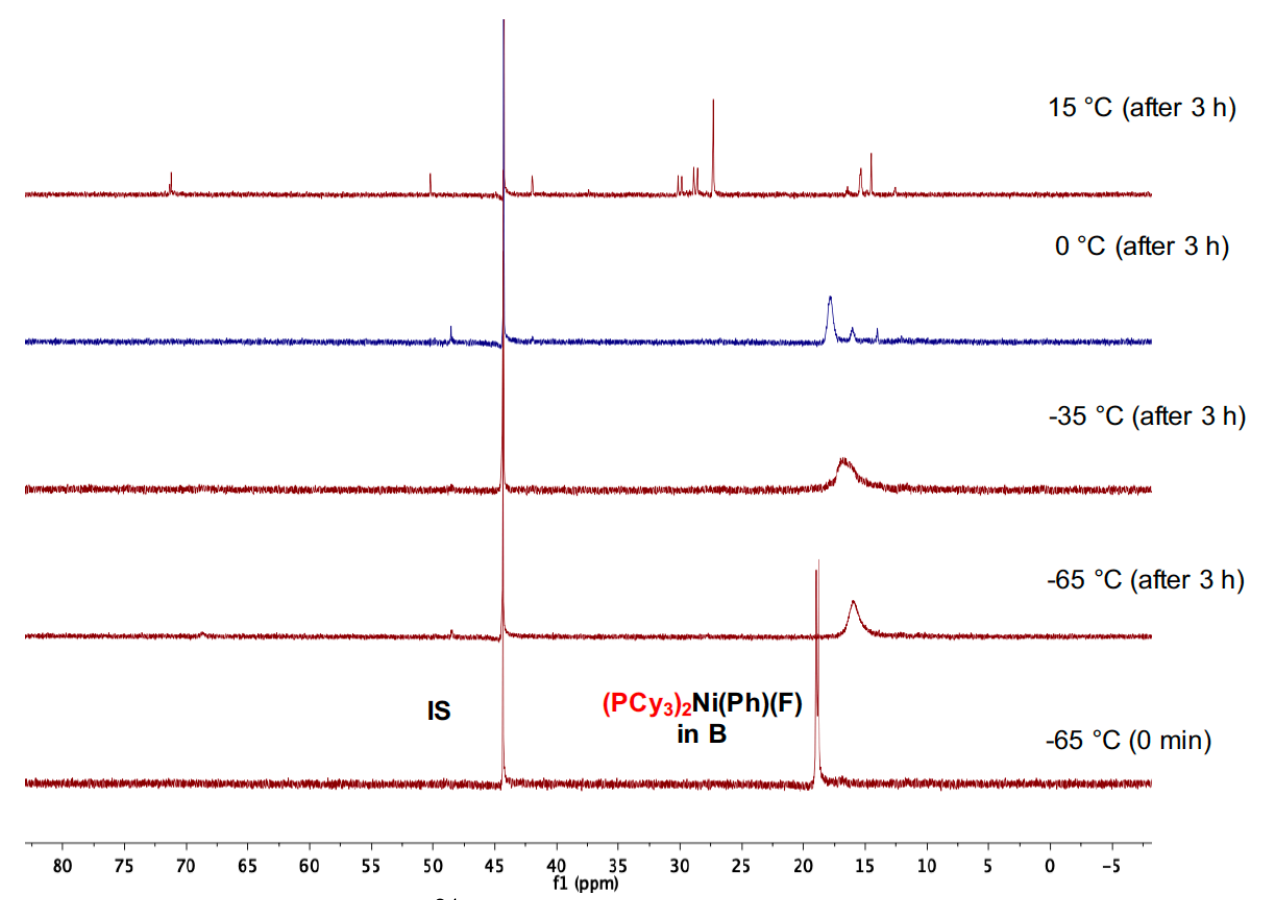

Figure S4A. Representative partial ${ }^{31} \mathrm{P} N \mathrm{NMR}$ spectra of the reaction of $\mathrm{B}$ and $\mathrm{B}_{2}(\mathrm{cat})_{2}$ to potentially observed intermediate $\mathbf{C}\left(\mathrm{PCy}_{3}\right)_{2} \mathrm{Ni}(\mathrm{Ph})$ (Bcat).

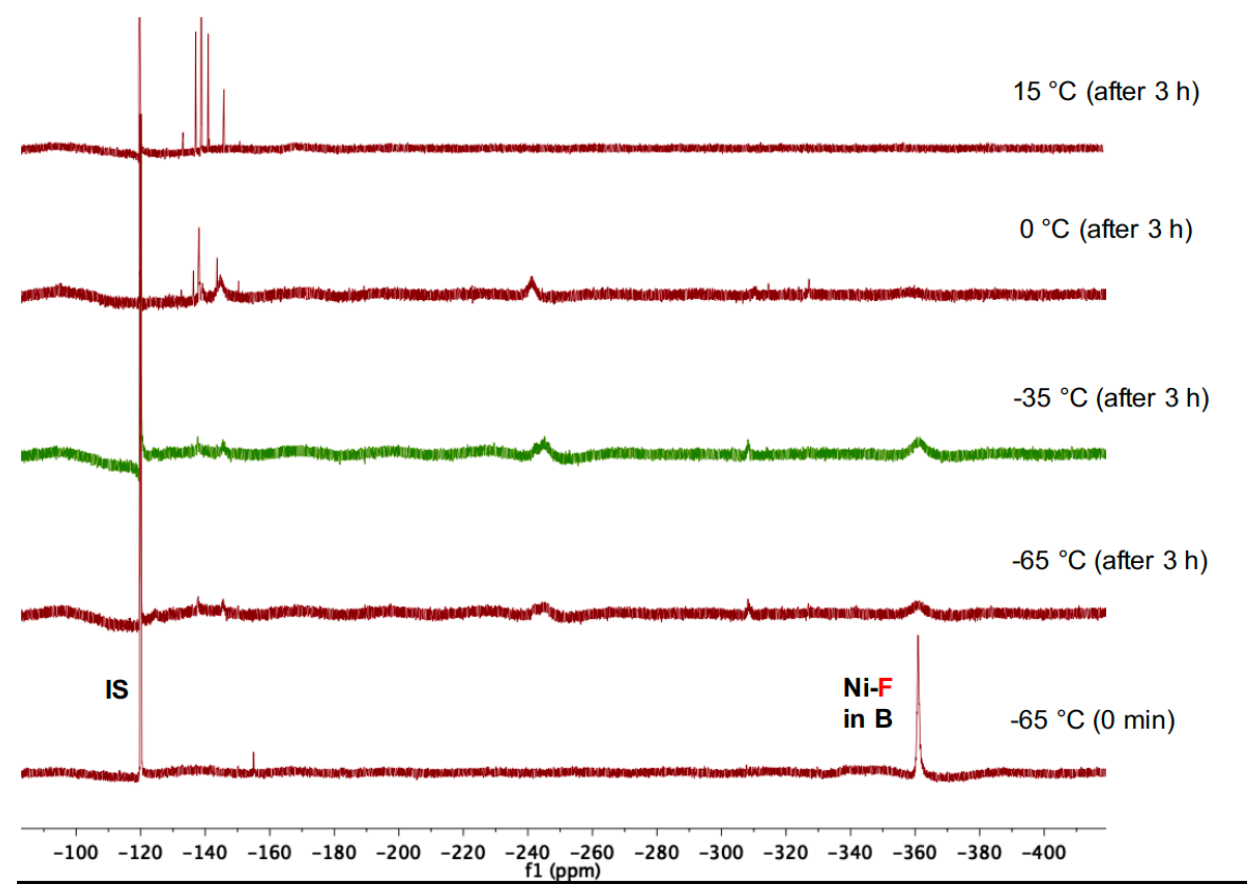

Figure S4B. Representative partial ${ }^{19} \mathrm{~F}$ NMR spectra of the reaction of $\mathrm{B}$ and $\mathrm{B}_{2}(\mathrm{cat})_{2}$ to potentially observed intermediate $\mathbf{C}\left(\mathrm{PCy}_{3}\right)_{2} \mathrm{Ni}(\mathrm{Ph})$ (Bcat). 


\section{Reaction of complexes B2 and B3 with 4-fluorophenyl boronic acid 1 and $B_{2}(\text { neo })_{2} 7$.}

In a glovebox, $(\mathrm{Ph}) \mathrm{Ni}\left(\mathrm{PC}_{3}\right)_{2}(\mathrm{Cl}) \mathbf{B 2}(7.1 \mathrm{mg}, 0.01 \mathrm{mmol}, 1.0$ equiv) was weighed into a $4 \mathrm{~mL}$ vial. 4-Fluorophenyl boronic acid 1 ( $0.012 \mathrm{mmol}, 1.2$ equiv) and 1,3,5-trimethoxybenzene ( $0.01 \mathrm{mmol}$, 1.0 equiv) were added from stock solutions in $d_{8}$-toluene resulting in a total volume of $0.6 \mathrm{~mL}$. The reaction mixture was stirred at room temperature for 15-30 s and immediately transferred to a J. Young NMR tube. The reaction was monitored by ${ }^{1} \mathrm{H}$ and ${ }^{31} \mathrm{P}$ NMR spectroscopy, as well as by GC/GCMS. A similar procedure was performed using $(\mathrm{Ph}) \mathrm{Ni}(\mathrm{PCy})_{2}(\mathrm{Br}) \mathbf{B} 3$ as well as $\mathrm{B}_{2}(\mathrm{neo})_{2} 7$ as a coupling partner at various temperatures. Results are summarized in Figure S5. Complexes B2 and B3 gave no observable reactivity with 1 and 7 at room temperature or $60^{\circ} \mathrm{C}$ in $1 \mathrm{~h}$. When the reactions were performed at $100{ }^{\circ} \mathrm{C}$, complexes B2 and B3 underwent complete decomposition to form $\left(\mathrm{PC}_{3}\right)_{2} \mathrm{Ni}(\mathrm{X})_{2}(\mathrm{X}=\mathrm{Br}$ or $\mathrm{Cl})$ and biphenyl.

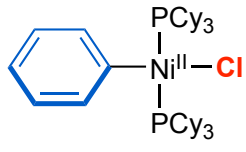

B2

or

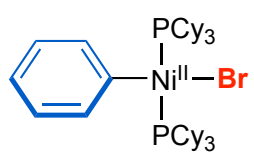

B3
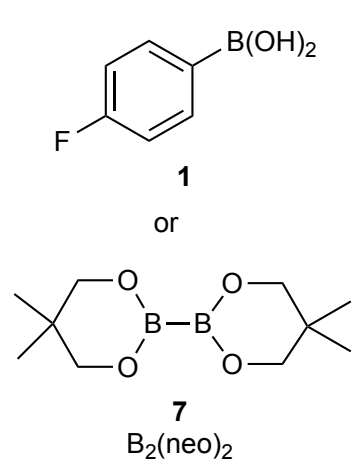

no reaction

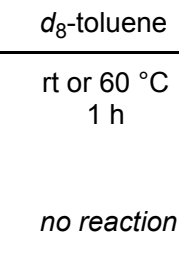

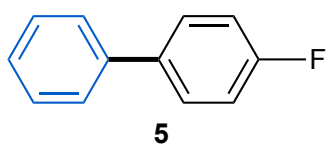

or

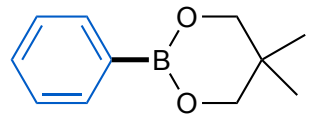

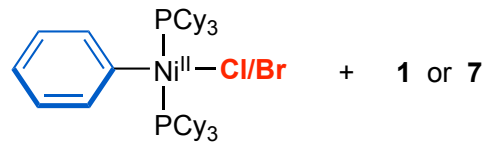

B2 or B3

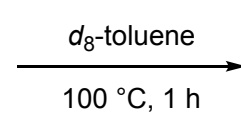

$100 \%$ conversion
$\left(\mathrm{PCy}_{3}\right)_{2} \mathrm{NiX}_{2}+$ $\mathrm{X}=\mathrm{Cl}$ or $\mathrm{Br}$<smiles>c1ccc(-c2ccccc2)cc1</smiles>

Figure S5. Reaction of nickel complex B2 and B3 with 1 and 7 


\section{Optimization of Ni-catalyzed decarbonylative borylation}

General procedure: In a nitrogen-filled glovebox, acid fluoride 10 (15.4 mg, $0.1 \mathrm{mmol}, 1.0$ equiv) was weighed into a $10 \mathrm{~mL}$ vial equipped with a $10 \mu \mathrm{m}$ magnetic stir bar (see Figure S6, set up C). A pre-mixed solution of $\mathrm{Ni}(\mathrm{cod})_{2}(0.01 \mathrm{mmol}, 0.1$ equiv) and ligand $(0.02 \mathrm{mmol}, 0.2$ equiv) in toluene $(0.2 \mathrm{~mL})$ and a stock THF solution $(0.1 \mathrm{~mL})$ of neopentylbenzene as internal standard ( $0.1 \mathrm{mmol}, 1$ equiv) were added. The diboron reagent $(0.2 \mathrm{mmol}, 2$ equiv) was added, and the vial was capped and removed from the glovebox. The reaction mixture was stirred at $115^{\circ} \mathrm{C}$ for $20 \mathrm{~h}$. The reaction mixture was cooled to room temperature, diluted with $\mathrm{DCM}$, filtered through a short plug of silica, and analyzed by GC.

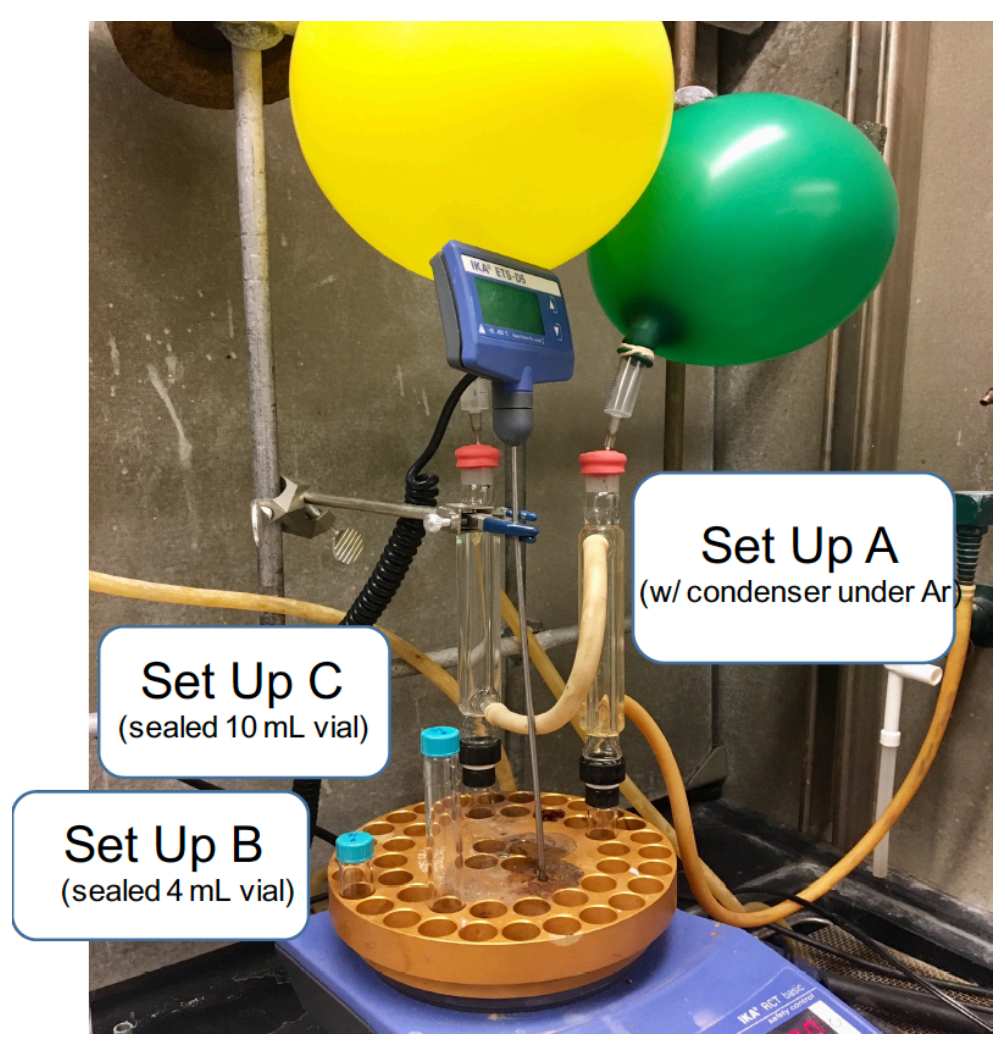

Figure S6. Reaction set up. Set up A: a $4 \mathrm{~mL}$ vial equipped with a condenser under Ar balloon. This is the set-up used for reactions at 0.3 to $0.5 \mathrm{mmol}$ scale (equivalent to 0.9 to $1.5 \mathrm{~mL}$ of THF as solvent). Set up B: a sealed $4 \mathrm{~mL}$ vial under $\mathrm{N}_{2}$ atmosphere. This set-up typically gives low selectivities for ketone versus biaryl product. Set up C: a sealed $10 \mathrm{~mL}$ vial under $\mathrm{N}_{2}$ atmosphere. This is the set-up used for optimization reactions and reactions at 0.1 to $0.2 \mathrm{mmol}$ scale (equivalent to 0.3 to $0.6 \mathrm{~mL}$ of THF as solvent). 
Table S2. Ligand screen
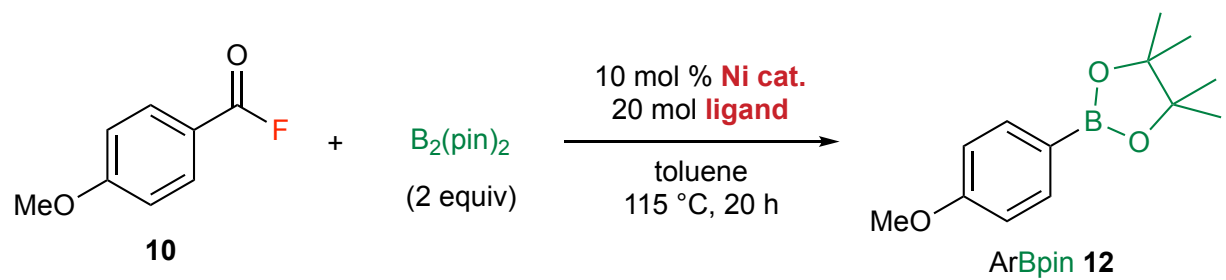

\begin{tabular}{ccc}
\hline entry & ligand & ayield of $\mathbf{1 2}(\%)$ \\
\hline 1 & none & $<2$ \\
2 & $\mathrm{PPh}_{2} \mathrm{Me}$ & 36 \\
3 & $\mathrm{PCy}_{3}$ & 86 \\
4 & $\mathrm{PEt}_{3}$ & 21 \\
5 & $\mathrm{P}^{t} \mathrm{Bu}_{3}$ & 5 \\
6 & $\mathrm{PPh}_{3}$ & 15 \\
7 & $\mathrm{XantPhos}$ & 12 \\
8 & $\mathrm{dppf}$ & 35 \\
9 & dppe & 5 \\
10 & iPr & $<2$ \\
11 & $\mathrm{SiPr}$ & $<2$ \\
12 & SPhos & 62 \\
13 & JohnPhos & 28 \\
14 & RuPhos & $<2$ \\
15 & BrettPhos & $<2$ \\
\hline
\end{tabular}

aYields determined by gas chromatography using neopentylbenzene as internal standard. 
Table S3A. Screen of nickel catalyst and diboron reagents.

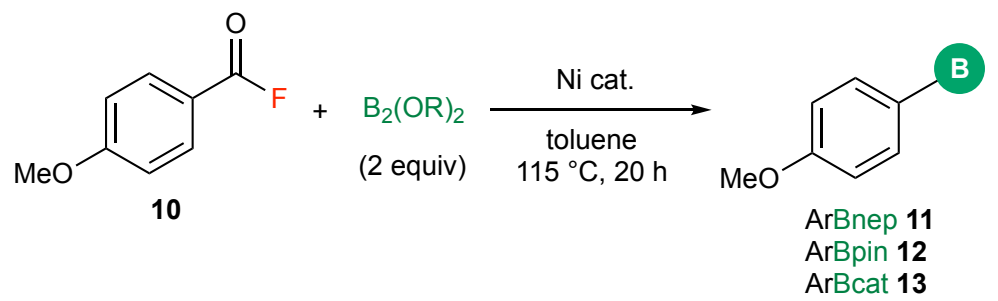

\begin{tabular}{ccc}
\hline entry & catalyst & ${ }^{a}$ yield of $11 / 12 / 13(\%)$ \\
\hline 1 & 10 mol \% Ni(cod) $)_{2}$ & $11,90 \%$ \\
2 & 20 mol\% PCy $_{3}$ & $12,86 \%$ \\
3 & 10 mol $\%$ & $13,83 \%$ \\
\hline 4 & catalyst B & $11,96 \%$ \\
5 & & $12,89 \%$ \\
6 & 10 mol $\%$ & $13,90 \%$ \\
\hline 8 & catalyst B1 & $11,90 \%$ \\
\hline 9 & 10 mol \% Ni(OAc) $)_{2}$ & $12,71 \%$ \\
\hline 10 & 20 mol\% PCy & $11,42 \%$ \\
\hline
\end{tabular}

aYields determined by gas chromatography using neopentylbenzene as internal standard. 
Table S3B. Screen of air-stable nickel pre-catalysts.

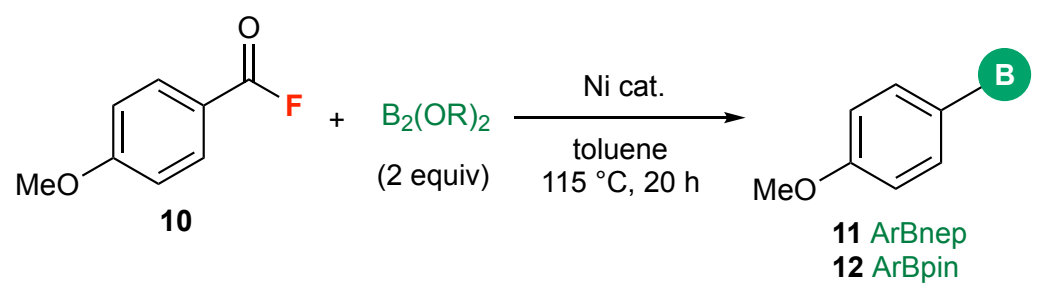

Condition A: use of $\mathrm{Ni}(\operatorname{cod})_{2}(10 \mathrm{~mol} \%)$, ligand $(20 \mathrm{~mol} \%)$

$\mathrm{PCy}_{3}$

ArBnep 11, 9\% (rt)

ArBnep $11,19 \%\left(60^{\circ} \mathrm{C}\right)$
$\mathrm{PCy}_{3}$

ArBnep 11, 90\%

ArBpin 12, 86\%
$\mathrm{PPh}_{2} \mathrm{Me}$

ArBnep 11, 36\%

ArBpin 12, 42\%

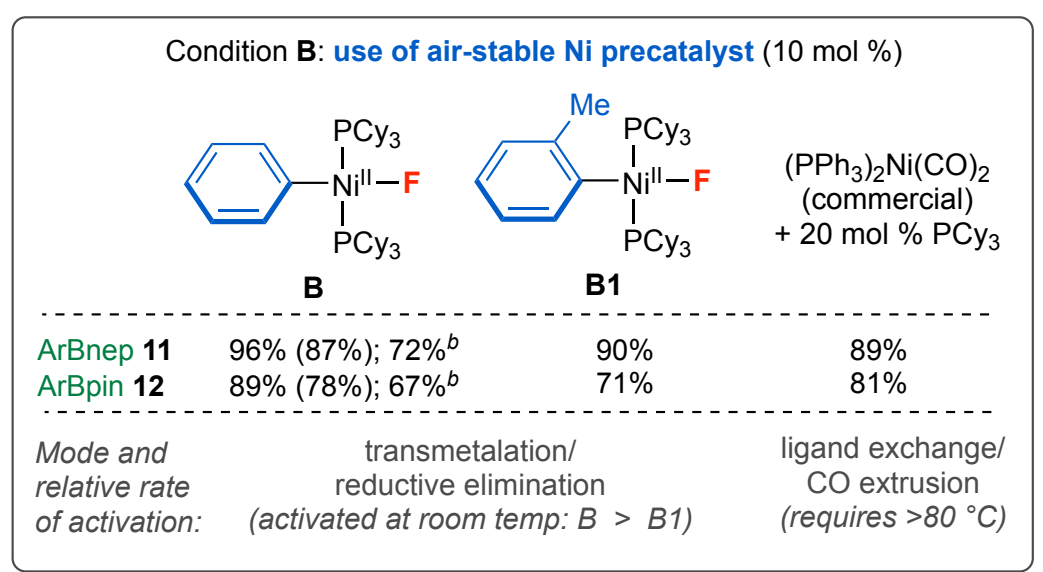

aYields determined by gas chromatography using neopentylbenzene as internal standard. Yields in parentheses are isolated yields. ${ }^{b}$ Yields from reactions performed on bench top. 
Table S4. Optimization of other conditions<smiles>COc1ccc(B2OCC(C)(C)CO2)cc1</smiles>

\begin{tabular}{ccc}
\hline entry & variation from scheme & yield of $\mathbf{1 2}(\%)$ \\
\hline 1 & none & 90 \\
$2^{b}$ & $5 \mathrm{~mol} \mathrm{\%} \mathrm{Ni(cod})_{2}, 10$ mol \% PCy & 70 \\
$3^{b}$ & $80^{\circ} \mathrm{C}$ & 44 \\
$4^{b}$ & $100{ }^{\circ} \mathrm{C}$ & 71 \\
5 & 1.5 equiv of $\mathrm{B}_{2}(\text { nep })_{2}$ & 79 \\
6 & 1.1 equiv of $\mathrm{B}_{2}(\text { nep })_{2}$ & 46 \\
9 & with $\mathrm{Na}_{2} \mathrm{CO}_{3}(2$ equiv) & 26 \\
10 & with $\mathrm{KF}(2$ equiv $)$ & 19 \\
$11^{c}$ & $\mathrm{Pd}(\mathrm{dba})_{2}$ instead of Ni(cod $)_{2}$ & $<2$ \\
\hline
\end{tabular}

aYields determined by gas chromatography using neopentylbenzene as internal standard. ${ }^{b} 10-20 \%$ of unreacted acid fluoride starting material was observed by ${ }^{19} \mathrm{~F}$ NMR spectroscopy. ${ }^{c}$ Mainly unreacted starting material remained. 


\section{Catalytic reactions at low temperatures and resting state analysis}

In a nitrogen-filled glovebox, benzoyl fluoride ( $0.2 \mathrm{mmol}, 1.0$ equiv) was weighed into a $10 \mathrm{~mL}$ vial equipped with a $10 \mu \mathrm{m}$ magnetic stir bar. A pre-mixed solution of $\mathrm{Ni}(\operatorname{cod})_{2}(0.02 \mathrm{mmol}, 0.1$ equiv) and ligand $\left(0.04 \mathrm{mmol}, 0.2\right.$ equiv) in toluene $(0.5 \mathrm{~mL})$ were added. The diboron reagent $\mathrm{B}_{2}(\mathrm{nep})_{2}$ ( $0.4 \mathrm{mmol}, 2$ equiv) was added, and the reaction mixture was stirred at room temperature for 1 min or until homogeneous. The solution was transferred to a J. Young tube and removed from the glovebox. The reaction mixture was analyzed by ${ }^{19} \mathrm{~F}$ and ${ }^{31} \mathrm{P}$ NMR at room temperature or 60 ${ }^{\circ} \mathrm{C}$ over time. Percent conversion was analyzed by ${ }^{19} \mathrm{~F}$ NMR and analysis of resting state was observed by ${ }^{31} \mathrm{P}$ NMR. Results are tabulated in Table S5.

In general, under these conditions, $\mathrm{Ni}(\mathrm{cod})\left(\mathrm{PCy}_{3}\right)_{2}$ was consumed within 30 min with concomitant conversion of benzoyl fluoride as determined by ${ }^{19} \mathrm{~F}$ NMR spectroscopy and GC analysis, respectively. After rapid initial conversion of acid fluoride to borylated products, $(\mathrm{PCy})_{2} \mathrm{Ni}(\mathrm{CO})_{2}$ was observed by ${ }^{31} \mathrm{P}$ NMR spectroscopy as the major detectable nickel-containing species (accounting for $>90 \%$ of the $\mathrm{Ni}$ ). This complex appears to be catalytically inactive at $60{ }^{\circ} \mathrm{C}$, as no additional conversion of starting material was observed after detection of this intermediate. In separate reactions, GC analysis at these time points showed product formations with yields remained constant from $3 \mathrm{~h}$ to $20 \mathrm{~h}$. For competency of a dicarbonyl nickel complex as precatalyst in catalysis, see Table S3B.

Table S5. Monitoring catalytic reactions at low temperatures.

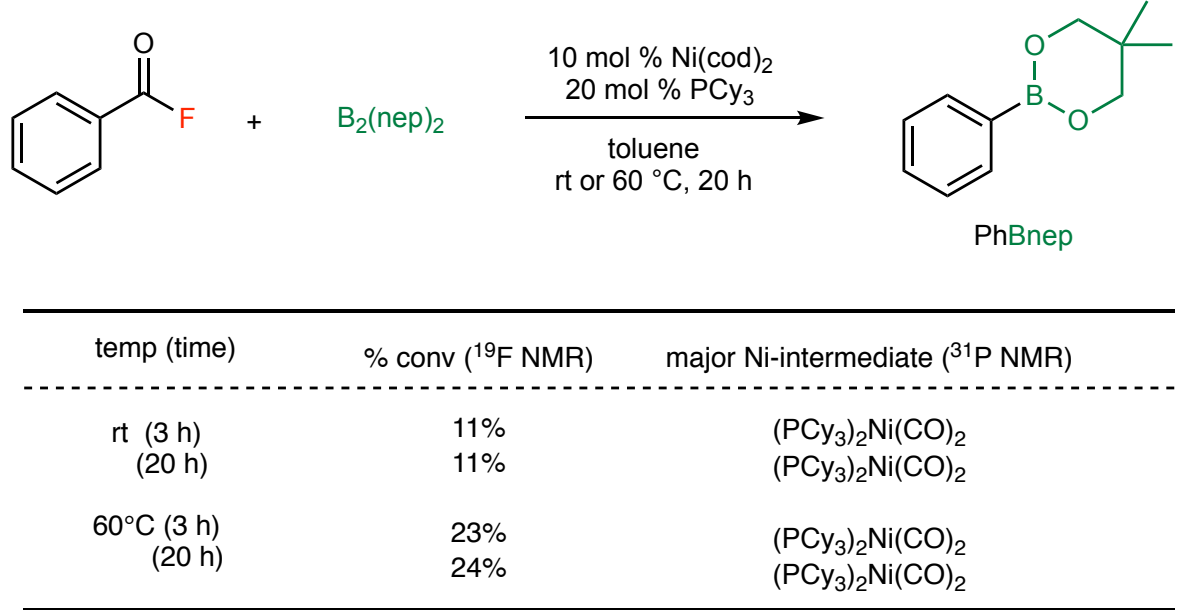

${ }^{a}$ Percent conversions were determined by ${ }^{19} \mathrm{~F}$ NMR using 4-fluorotoluene as internal standard. Intermediates were observed ${ }^{31} \mathrm{P}$ NMR spectroscopy, the major intermediate $\left(\mathrm{PCy}_{3}\right)_{2} \mathrm{Ni}(\mathrm{CO})_{2}$ was estimated to be $>90 \%$ based on total integration of all ${ }^{31} \mathrm{P}$ signals. 


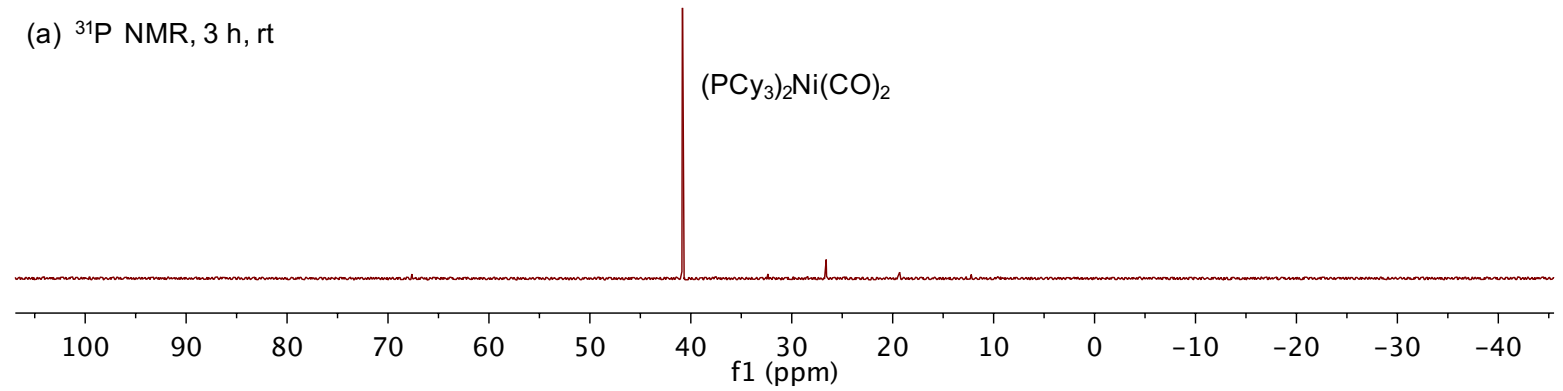

(b) ${ }^{31} \mathrm{P} \mathrm{NMR}, 3 \mathrm{~h}, 60^{\circ} \mathrm{C}$

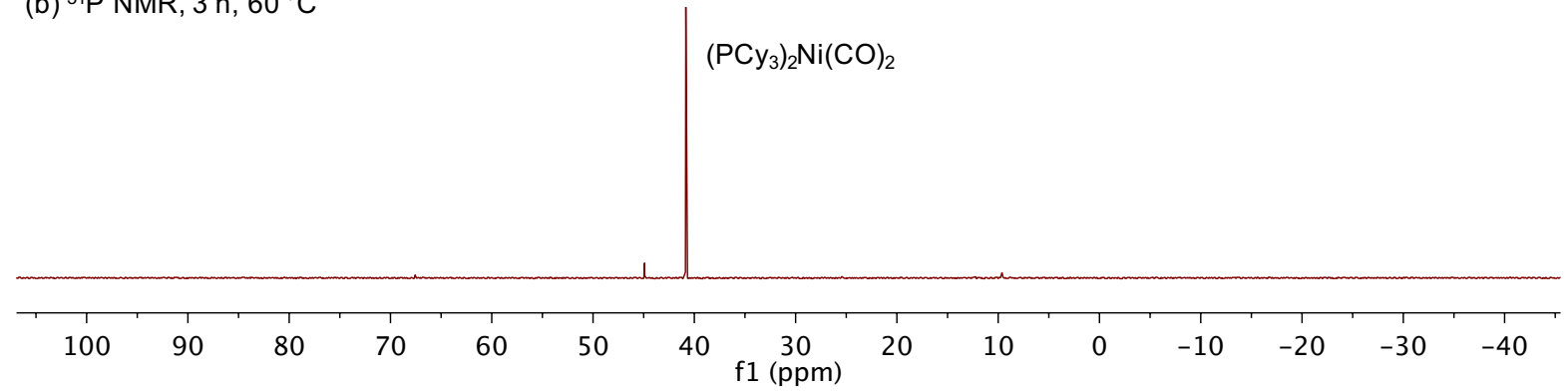

Figure S7. Representative ${ }^{31} \mathrm{P}$ NMR spectra of the reactions at it and $60{ }^{\circ} \mathrm{C}$ after $3 \mathrm{~h}$. No significant changes on ${ }^{31} \mathrm{P}$ NMR and ${ }^{19} \mathrm{~F} \mathrm{NMR}$ (not shown) spectra were observed after $20 \mathrm{~h}$. 


\section{Ni-catalyzed decarbonylative borylation via in situ formation of acid fluoride}

In situ reaction, general procedure: In a nitrogen-filled glovebox, probenecid $(0.1 \mathrm{mmol}, 1$ equiv), tetramethylfluoroformamidinium hexafluorophosphate (TFFH) $(0.1 \mathrm{mmol}, 1$ equiv), and base $(0.1 \mathrm{mmol}, 1$ equiv) were weighed into a $10 \mathrm{~mL}$ tall vial equipped with a $10 \mu \mathrm{m}$ magnetic stir bar. THF $(0.2 \mathrm{~mL})$ was added, and the reaction mixture was stirred at room temperature for 15 min. A pre-mixed solution of $\mathrm{Ni}(\mathrm{cod})_{2}\left(0.01 \mathrm{mmol}, 0.1\right.$ equiv) and $\mathrm{PCy}_{3}(0.02 \mathrm{mmol}, 0.2$ equiv) in THF $(0.1 \mathrm{~mL})$ and a THF stock solution $(0.1 \mathrm{~mL})$ of neopentylbenzene as internal standard $(0.1$ mmol, 1 equiv) were added. The diboron reagent $(0.4 \mathrm{mmol}, 2$ equiv) was added, and the vial was capped and removed from the glovebox. The reaction was stirred at $115^{\circ} \mathrm{C}$ for $20 \mathrm{~h}$. The reaction mixture was cooled to room temperature, and then diluted with dichloromethane and analyzed by GC.

Table S6. Ni-catalyzed decarbonylative borylation via in situ formation of acid fluoride.

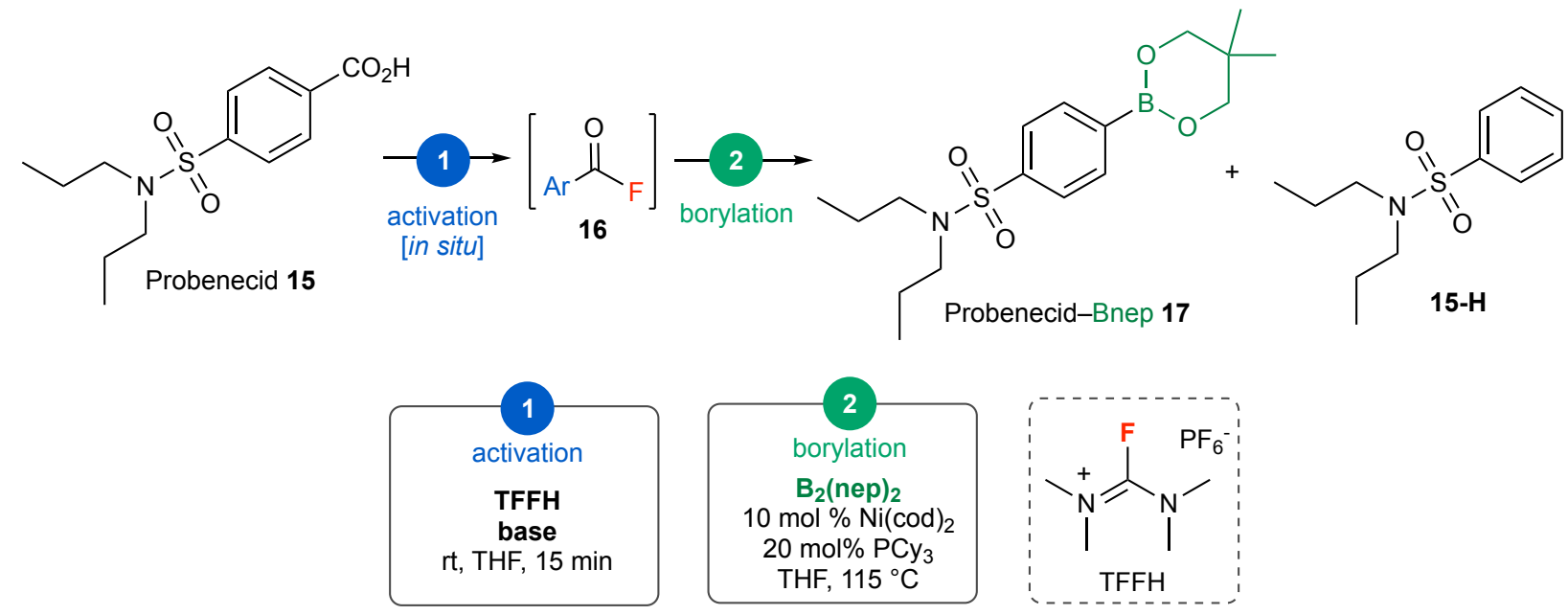

\begin{tabular}{cccc}
\hline entry & base (1.0 equiv) & ${ }^{a}$ yield of $\mathbf{1 7}(\%)$ & ${ }^{a}$ yield of 15-H (\%) \\
\hline 1 & DIPEA & 20 & 65 \\
2 & $\mathrm{Et}_{3} \mathrm{~N}$ & 11 & 70 \\
3 & pyridine & 17 & 70 \\
3 & proton sponge & $81(76)^{c}$ & $<2$ \\
$4^{b}$ & $\mathrm{NaH}$ & 0 & 0 \\
5 & $\mathrm{KH}$ & 12 & 25 \\
$6^{b}$ & $\mathrm{NaO}{ }^{t} \mathrm{Bu}$ & 0 & 0 \\
7 & proton sponge (1.2 equiv) & 78 & 5 \\
\hline
\end{tabular}

${ }^{a} \mathrm{GC}$ yields determined using neopentylbenzene as internal standard. ${ }^{b}$ The in situ formation of acid fluoride was found to be inefficient as observed by ${ }^{19} \mathrm{~F}$ NMR spectroscopic analysis of the reaction mixture after activation step. ${ }^{C}$ Isolated yield from a $0.3 \mathrm{mmol}$ scale. 


\section{Selectivity studies}

In a nitrogen-filled glovebox, acid fluoride 10 or $14(0.1 \mathrm{mmol}, 1$ equiv) was weighed into a $10 \mathrm{~mL}$ vial equipped with a $10 \mu \mathrm{m}$ magnetic stir bar (see Figure S6, set up C). Catalyst B $(0.01 \mathrm{mmol}$, 0.1 equiv) in toluene $(0.2 \mathrm{~mL})$ and a THF stock solution $(0.1 \mathrm{~mL})$ of neopentylbenzene as internal standard ( $0.1 \mathrm{mmol}, 1$ equiv) were added. The diboron reagent $(0.2 \mathrm{mmol}, 2.0$ equiv) was added, and the vial was capped and removed from the glovebox. The reaction mixture was stirred at 115 ${ }^{\circ} \mathrm{C}$ for $20 \mathrm{~h}$. The reaction mixture was cooled to room temperature, diluted with DCM, filtered through a short plug of silica, and analyzed by GC. Using acid fluorides 10, 14, and benzoyl fluoride as substrates the yields and selectivities obtained from the reaction with various diboron reagents were compared. Selectivity results are summarized in Figure S8.
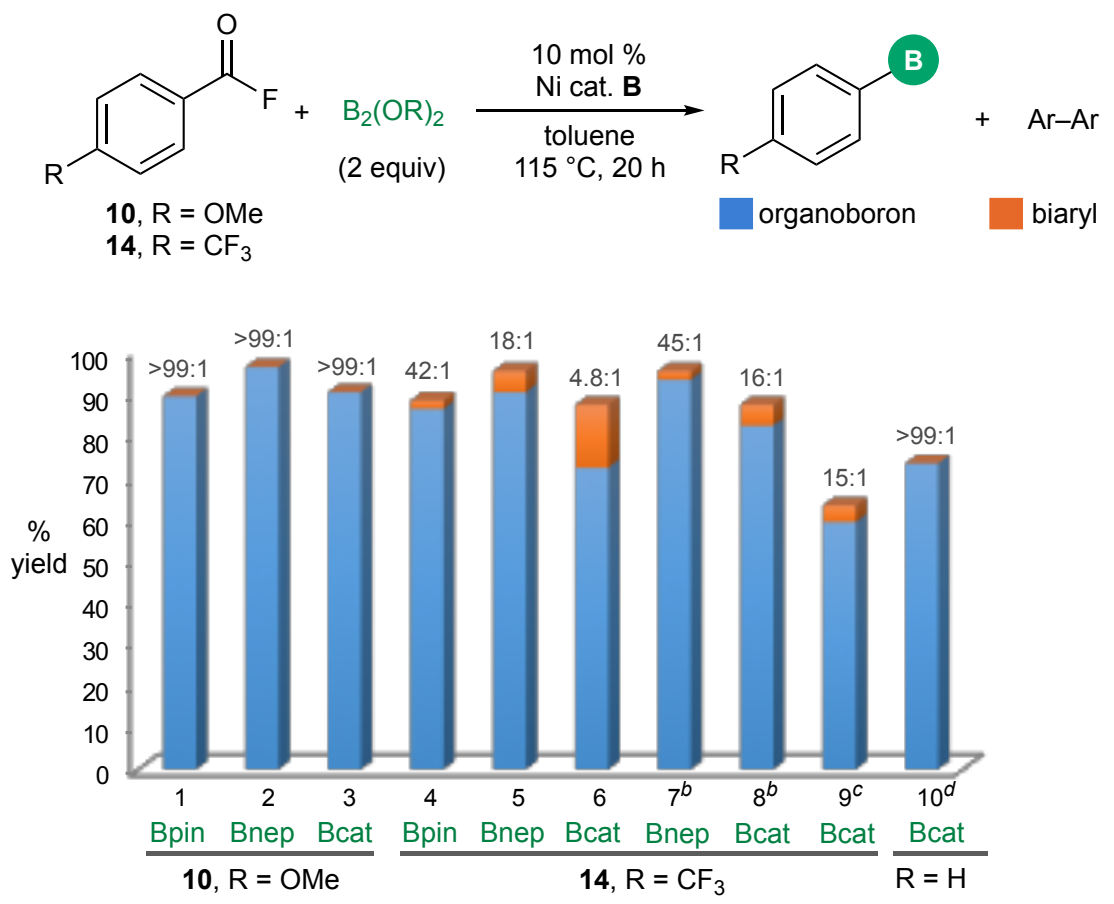

Figure S8. Comparison of selectivities obtained from the reaction of aroyl fluorides 10, 14, and

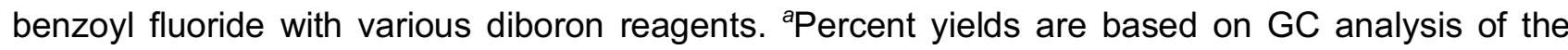
reaction crude mixture. ${ }^{b}$ Reactions were performed using 3 equivalents of diboron reagents. ${ }^{c}$ Reaction was performed at $90{ }^{\circ} \mathrm{C}$. $\left.{ }^{d} 10 \mathrm{~mol} \mathrm{\%} \mathrm{Ni(cod)}\right)_{2}$ and $20 \mathrm{~mol} \% \mathrm{PCy}_{3}$ were used instead of catalyst B 
Decarbonylation method:

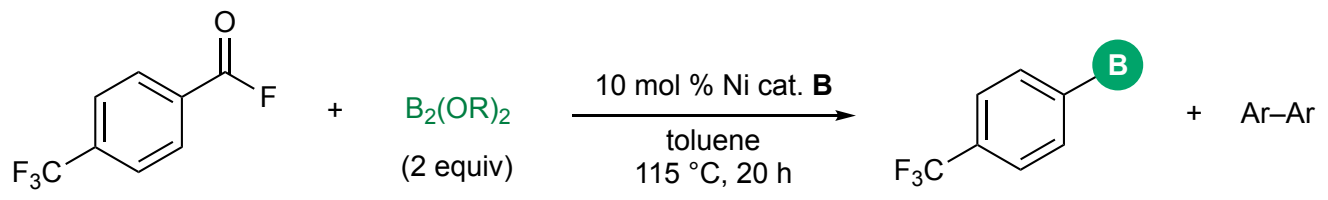

Miyaura borylation conditions:<smiles></smiles>

\begin{tabular}{cccc}
\hline & $\begin{array}{c}\text { Decarbonylation method } \\
\% \text { yield (ArB:Ar-Ar) }\end{array}$ & $\begin{array}{c}\text { Miyaura conditions } \\
\% \text { yield }(\mathrm{ArB}: \mathrm{Ar}-\mathrm{Ar})^{a}\end{array}$ & $\begin{array}{c}\text { Miyaura conditions } \\
{\left[2 \text { equiv of } \mathrm{B}_{2}(\mathrm{OR})_{2}\right]} \\
\% \text { yield }(\mathrm{ArB}: \mathrm{Ar}-\mathrm{Ar})^{a}\end{array}$ \\
\hline $\mathrm{B}_{2}(\mathrm{pin})_{2}$ & $86 \%(42: 1)$ & $90 \%(17: 1)$ & $85 \%(17: 1)$ \\
$\mathrm{B}_{2}(\mathrm{nep})_{2}$ & $90 \%(18: 1)$ & $93 \%(15: 1)$ & $95 \%(18: 1)$ \\
$\mathrm{B}_{2}(\text { cat })_{2}$ & $72 \%(4.8: 1)$ & $0 \%(--)$ & $0 \%(--)$ \\
\hline
\end{tabular}

a\% GC yield (selectivity of ArB:biaryl)

Figure S9. Comparison of selectivities obtained from the decarbonylative borylation of aroyl fluoride 14 versus Pd-catalyzed borylation of 4-bromodenzotrifluoride under Miyaura conditions. ${ }^{4}{ }^{4}$ Yields and selectivities are based on $\mathrm{GC}$ analysis of the reaction crude mixture 


\section{Synthesis of Acid Fluorides}<smiles>O=C(O)c1ccccc1</smiles>

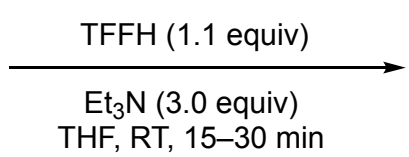<smiles>O=C(F)c1ccccc1</smiles>

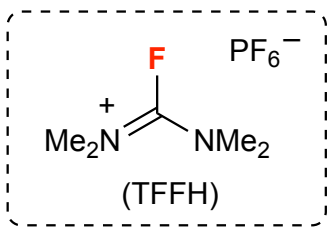

General procedure for the synthesis of acid fluorides from carboxylic acids (Method A): Based on a modified literature procedure,,$^{5,6}$ a $20 \mathrm{~mL}$ vial equipped with a magnetic stir bar was charged with carboxylic acid (2.0 mmol, 1.0 equiv), TFFH (2.2 mmol, 1.1 equiv), and triethylamine $(6.0 \mathrm{mmol}, 3.0$ equiv) in $\mathrm{DCM}(10 \mathrm{~mL})$. The reaction mixture was stirred at room temperature for $1 \mathrm{~h}$. The reaction mixture was diluted with $\mathrm{DCM}(10 \mathrm{~mL})$ and washed with ice-cold water $(10 \mathrm{~mL}$ $x 2$ ). The organic extracts were collected, was dried over $\mathrm{Na}_{2} \mathrm{SO}_{4}$, and concentrated in vacuo. The crude product was purified by flash column chromatography on silica gel using EtOAc in hexanes.<smiles>O=C(Cl)c1ccccc1</smiles>

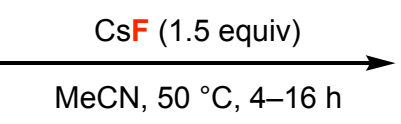<smiles>O=C(F)c1ccccc1</smiles>

General procedure for the synthesis of acid fluorides from acid chlorides (Method B): Based on a modified literature procedure, ${ }^{2,6}$ a $20 \mathrm{~mL}$ vial equipped with a magnetic stir bar was charged with $\mathrm{CsF}$ (3.0 mmol, 1.5 equiv) and the appropriate carboxylic acid chloride $(2.0 \mathrm{mmol}$, 1.0 equiv) in anhydrous $\mathrm{MeCN}(6 \mathrm{~mL})$. The reaction mixture was stirred at $50{ }^{\circ} \mathrm{C}$ until complete conversion (usually 4 to $16 \mathrm{~h}$ ). The resulting suspension was allowed to settle, and the MeCN solution was decanted from the precipitate and then filtered. The precipitate was washed with MeCN (6 mL), and the washes were filtered. The combined MeCN washes were concentrated in vacuo. Based on ${ }^{1} \mathrm{H}$ NMR spectroscopic analysis, the resulting product was typically $\sim 95 \%$ pure and was thus used without further purification. If purification was necessary, silica gel column chromatography was performed using EtOAc in hexanes.

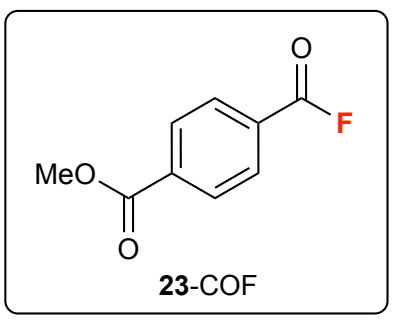


Methyl 4-(fluorocarbonyl)benzoate (23-COF). Following the general procedure (Method B) using methyl 4-(chlorocarbonyl)benzoate, methyl 4-(fluorocarbonyl)benzoate (23-COF) was obtained as a white solid (328 mg, 90\% yield): $\mathrm{mp} 70-71{ }^{\circ} \mathrm{C} ;{ }^{1} \mathrm{H}$ NMR $\left(\mathrm{CDCl}_{3}, 500 \mathrm{MHz}\right) \delta 8.13$ $(\mathrm{d}, J=8.7 \mathrm{~Hz}, 2 \mathrm{H}), 8.07$ (d, $J=8.7 \mathrm{~Hz}, 2 \mathrm{H}), 3.94(\mathrm{~s}, 3 \mathrm{H}) ;{ }^{13} \mathrm{C} \mathrm{NMR}\left(\mathrm{CDCl}_{3}, 126 \mathrm{MHz}\right) \delta 165.70$, $156.62(\mathrm{~d}, J=345.7 \mathrm{~Hz}), 136.15,131.46$ (d, $J=3.8 \mathrm{~Hz}), 130.17,128.69$ (d, $J=61.7 \mathrm{~Hz}), 52.81$; ${ }^{19} \mathrm{~F} \mathrm{NMR}\left(\mathrm{CDCl}_{3}, 471 \mathrm{MHz}\right) \delta$ 19.88; HRMS (El) calcd for $\mathrm{C}_{9} \mathrm{H}_{7} \mathrm{FO}_{3}[\mathrm{M}]^{+} \mathrm{m} / \mathrm{z}$ 182.0379, found 182.0381.

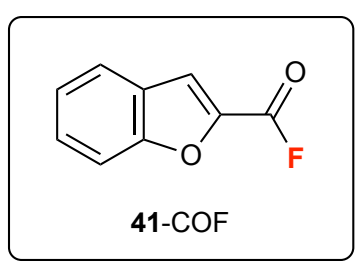

Benzofuran-2-carbonyl fluoride (41-COF). Following the general procedure (Method B) using commercial benzofuran-2-carbonyl chloride, 41-COF was obtained as a light yellow solid (298 mg, $91 \%$ yield): $\mathrm{mp} 77-78{ }^{\circ} \mathrm{C} ;{ }^{1} \mathrm{H}$ NMR $\left(\mathrm{CDCl}_{3}, 500 \mathrm{MHz}\right) \delta 7.76$ (multiple peaks, $2 \mathrm{H}$ ), 7.67-7.60 $(\mathrm{m}, 1 \mathrm{H}), 7.58-7.54(\mathrm{~m}, 1 \mathrm{H}), 7.42-7.35(\mathrm{~m}, 1 \mathrm{H}) ;{ }^{13} \mathrm{C} \mathrm{NMR}\left(\mathrm{CDCl}_{3}, 126 \mathrm{MHz}\right) \delta 157.11(\mathrm{~d}, J=2.4$ $\mathrm{Hz}$ ), 149.61 (d, $J=329.9 \mathrm{~Hz}$ ), 140.04 (d, $J=89.6 \mathrm{~Hz}), 129.71,126.48,124.79,123.74,119.54$, 112.88; ${ }^{19} \mathrm{~F}$ NMR $\left(\mathrm{CDCl}_{3}, 471 \mathrm{MHz}\right) \delta 17.38$; HRMS (EI) calcd for $\mathrm{C}_{9} \mathrm{H}_{5} \mathrm{FO}_{2}[\mathrm{M}]^{+} \mathrm{m} / \mathrm{z} 164.0274$, found 164.0279 .

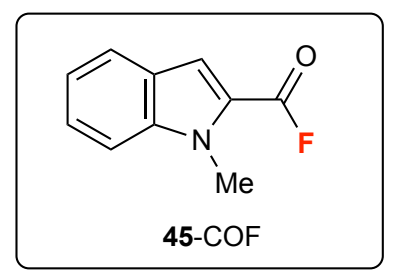

1-Methyl-1H-indole-2-carbonyl fluoride (45-COF). Following the general procedure (Method A) using commercial 1-methyl-1H-indole-2-carboxylic acid, 45-COF was obtained as a white solid (301 mg, 85\% yield): $\mathrm{mp} 77-78{ }^{\circ} \mathrm{C} ;{ }^{1} \mathrm{H}$ NMR $\left(\mathrm{CDCl}_{3}, 700 \mathrm{MHz}\right) \delta 7.73$ (dd, $\left.J=8.1,0.8 \mathrm{~Hz}, 1 \mathrm{H}\right)$, $7.49(\mathrm{~s}, 1 \mathrm{H}), 7.47-7.44(\mathrm{~m}, 1 \mathrm{H}), 7.43-7.40(\mathrm{~m}, 1 \mathrm{H}), 7.23-7.12(\mathrm{~m}, 1 \mathrm{H}), 4.07(\mathrm{~s}, 3 \mathrm{H}) ;{ }^{13} \mathrm{C}$ NMR $\left(\mathrm{CDCl}_{3}, 100 \mathrm{MHz}\right) \delta 152.26(\mathrm{~d}, J=328.5 \mathrm{~Hz}), 141.21(\mathrm{~d}, J=5.0 \mathrm{~Hz}), 127.23,125.77,123.60$, $121.97(\mathrm{~d}, J=81.9 \mathrm{~Hz}), 121.63,115.16(\mathrm{~d}, \mathrm{~J}=2.6 \mathrm{~Hz}), 110.75,31.68 ;{ }^{19} \mathrm{~F} \mathrm{NMR}\left(\mathrm{CDCl}_{3}, 377 \mathrm{MHz}\right)$ $\delta$ 22.20; HRMS (El) calcd for $\mathrm{C}_{10} \mathrm{H}_{8} \mathrm{FNO}[\mathrm{M}]^{+} \mathrm{m} / \mathrm{z} 177.0590$, found 177.0588 . 


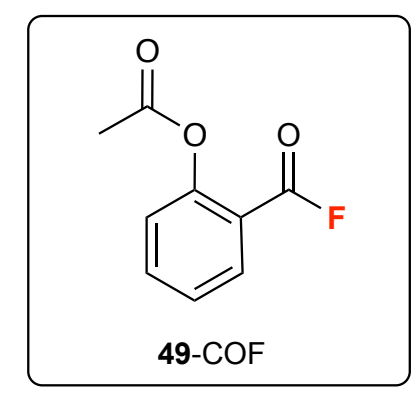

2-(Fluorocarbonyl)phenyl acetate (49-COF). Following the general procedure (Method A) using aspirin, 49-COF was obtained after column chromatography as a colorless oil $(255 \mathrm{mg}, 70 \%$ yield): ${ }^{1} \mathrm{H}$ NMR $\left(\mathrm{CDCl}_{3}, 700 \mathrm{MHz}\right) \delta 8.04(\mathrm{~m}, 1 \mathrm{H}), 7.71(\mathrm{~m}, 1 \mathrm{H}), 7.39(\mathrm{t}, J=7.8 \mathrm{~Hz}, 1 \mathrm{H}), 7.20(\mathrm{~d}$, $J=7.8 \mathrm{~Hz}, 1 \mathrm{H}), 2.37(\mathrm{~s}, 3 \mathrm{H}) ;{ }^{13} \mathrm{C} \mathrm{NMR}\left(\mathrm{CDCl}_{3}, 126 \mathrm{MHz}\right) \delta 169.42,154.21(\mathrm{~d}, J=343.3 \mathrm{~Hz})$, $152.64(\mathrm{~d}, J=5.3 \mathrm{~Hz}$ ), 136.72, 133.21 (d, $J=2.0 \mathrm{~Hz}$ ), 126.67, 124.62 (d, $J=3.5 \mathrm{~Hz}$ ), 118.34 (d, $J=60.3 \mathrm{~Hz}), 21.02 ;{ }^{19} \mathrm{~F} \mathrm{NMR}\left(\mathrm{CDCl}_{3}, 376 \mathrm{MHz}\right) \delta 28.31$; HRMS (EI) calcd for $\mathrm{C}_{9} \mathrm{H}_{7} \mathrm{FNO}_{3}[\mathrm{M}]^{+} \mathrm{m} / \mathrm{z}$ 182.1504, found 182.1505 .

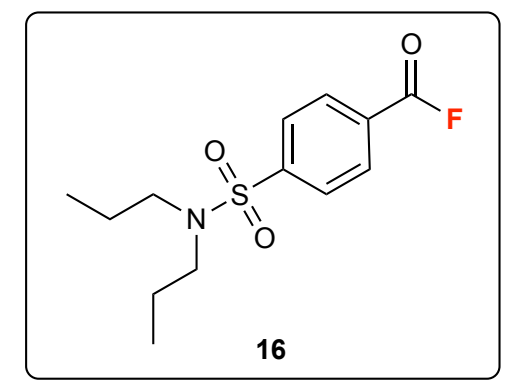

4-(N,N-Dipropylsulfamoyl)benzoyl fluoride (16). Following the general procedure (Method A) using probenecid, 16 was obtained after column chromatography as a white solid (470 $\mathrm{mg}, 82 \%$ yield): mp 61-62 ${ }^{\circ} \mathrm{C} ;{ }^{1} \mathrm{H}$ NMR $\left(\mathrm{CDCl}_{3}, 700 \mathrm{MHz}\right) \delta 8.15(\mathrm{~d}, \mathrm{~J}=8.1 \mathrm{~Hz}, 2 \mathrm{H}), 7.94(\mathrm{~d}, J=8.1 \mathrm{~Hz}$, $2 \mathrm{H}), 3.49-2.65(\mathrm{~m}, 4 \mathrm{H}), 1.53(\mathrm{q}, J=7.5 \mathrm{~Hz}, 4 \mathrm{H}), 0.85(\mathrm{t}, J=7.4 \mathrm{~Hz}, 6 \mathrm{H}) ;{ }^{13} \mathrm{C}$ NMR $\left(\mathrm{CDCl}_{3}, 100\right.$ $\mathrm{MHz}) \delta 156.16(\mathrm{~d}, J=345.8 \mathrm{~Hz}), 146.86,132.18(\mathrm{~d}, J=3.2 \mathrm{~Hz}), 128.23(\mathrm{~d}, J=62.5 \mathrm{~Hz}), 127.68$, $50.08,22.08,11.25 ;{ }^{19} \mathrm{~F} \mathrm{NMR}\left(\mathrm{CDCl}_{3}, 377 \mathrm{MHz}\right) \delta 20.19$; HRMS (El) calcd for $\mathrm{C}_{13} \mathrm{H}_{18} \mathrm{FNO}_{3} \mathrm{~S}[\mathrm{M}]^{+}$ $\mathrm{m} / \mathrm{z} 287.0991$, found 287.0994. 
XIV. Scope of Ni-Catalyzed Decarbonylative Borylation

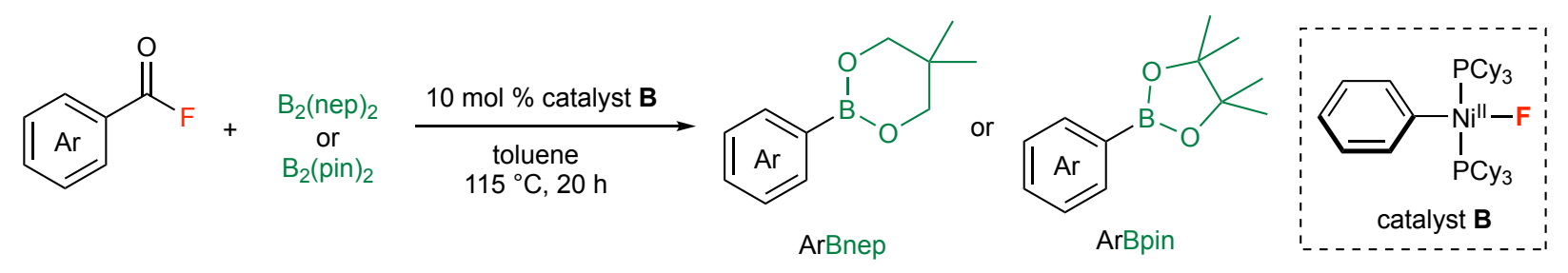

General procedure for decarbonylative borylation reaction (Method $A$, from acid fluoride): In a nitrogen-filled glovebox, catalyst $\mathbf{B}(0.03 \mathrm{mmol}, 0.1$ equiv) was weighed into a $10 \mathrm{~mL}$ tall vial equipped with a $10 \mu \mathrm{m}$ magnetic stir bar. The acid fluoride $(0.3 \mathrm{mmol}, 1$ equiv), diboron reagent ( $0.6 \mathrm{mmol}, 2$ equiv), and toluene $(0.6 \mathrm{~mL})$ were added. The reaction mixture was capped and removed from the glovebox. The reaction mixture was stirred at $115^{\circ} \mathrm{C}$ for $16 \mathrm{~h}$. The reaction was then cooled to room temperature, and $\mathrm{Et}_{2} \mathrm{O}(10 \mathrm{~mL})$ and saturated $\mathrm{NaHCO}_{3}(10 \mathrm{~mL})$ were added. The organic layer was collected, and the aqueous solution was further extracted with $\mathrm{Et}_{2} \mathrm{O}(2 \mathrm{x}$ $10 \mathrm{~mL}$ ). The combined organic extracts were dried over $\mathrm{Na}_{2} \mathrm{SO}_{4}$ and concentrated in vacuo. The crude product was purified by flash column chromatography on silica gel using EtOAc in hexanes.

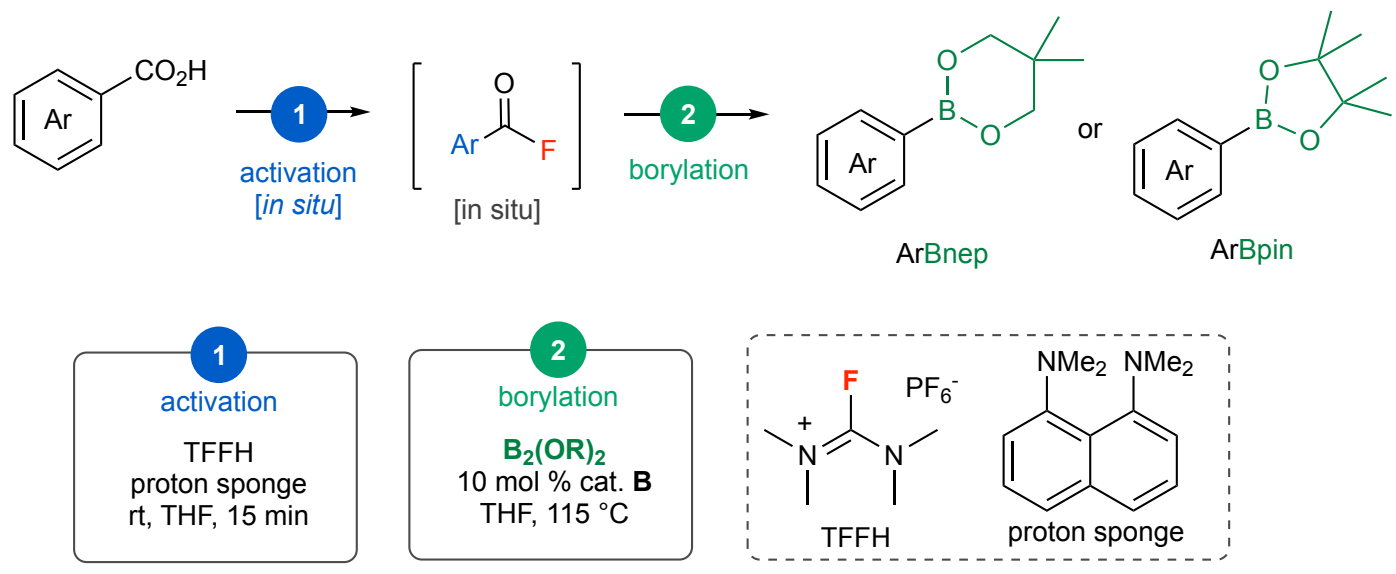

General procedure for decarbonylative borylation reaction (Method $B$, in situ, from carboxylic acid): In a nitrogen-filled glovebox, carboxylic acid ( $0.3 \mathrm{mmol}, 1$ equiv), TFFH (0.3 mmol, 1 equiv), and proton sponge $(0.3 \mathrm{mmol}, 1$ equiv) were weighed into a $10 \mathrm{~mL}$ tall vial equipped with a $10 \mu \mathrm{m}$ magnetic stir bar. THF $(0.5 \mathrm{~mL})$ was added, and the reaction mixture was stirred at room temperature for 15 to $30 \mathrm{~min}$. Catalyst $\mathbf{B}(0.03 \mathrm{mmol}, 0.1$ equiv) and diboron reagent $(0.6 \mathrm{mmol}, 2$ equiv) were added. The vial was capped and removed from the glovebox. The reaction mixture was stirred at $115^{\circ} \mathrm{C}$ for $16 \mathrm{~h}$. The reaction was then cooled to room temperature, and $\mathrm{Et}_{2} \mathrm{O}(10 \mathrm{~mL})$ and saturated $\mathrm{NaHCO}_{3}(10 \mathrm{~mL})$ were added. The organic layer was collected, and the aqueous solution was further extracted with $\mathrm{Et}_{2} \mathrm{O}(2 \times 10 \mathrm{~mL})$. The combined organic extracts were dried over $\mathrm{Na}_{2} \mathrm{SO}_{4}$ and concentrated in vacuo. The crude product was purified by flash column chromatography on silica gel using EtOAc in hexanes. 


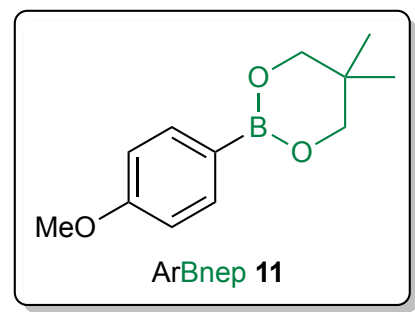

2-(4-Methoxyphenyl)-5,5-dimethyl-1,3,2-dioxaborinane (11). Followed Method A using aroyl fluoride ${ }^{6} 10$ and $B_{2}(\text { nep) })_{2}$. Purification by flash chromatography on silica gel (hexanes/EtOAc, 95:5) afforded 11 as a colorless solid (53 mg, 80\% yield): $\mathrm{mp} 57-58{ }^{\circ} \mathrm{C} ;{ }^{1} \mathrm{H} \mathrm{NMR}\left(500 \mathrm{MHz}, \mathrm{CDCl}_{3}\right)$ $\delta 7.75(\mathrm{~d}, J=8.5 \mathrm{~Hz}, 2 \mathrm{H}), 6.89(\mathrm{~d}, \mathrm{~J}=8.5 \mathrm{~Hz}, 2 \mathrm{H}), 3.82(\mathrm{~s}, 3 \mathrm{H}), 3.75(\mathrm{~s}, 4 \mathrm{H}), 1.02(\mathrm{~s}, 6 \mathrm{H}) ;{ }^{13} \mathrm{C}$ NMR $\left(126 \mathrm{MHz} \mathrm{CDCl}_{3}\right) \delta 161.96,135.72,113.36,72.48,55.27,32.12,22.15$, the carbon attached to boron was not observed due to quadrupolar coupling; ${ }^{11} \mathrm{~B} \mathrm{NMR}\left(\mathrm{CDCl}_{3}, 128 \mathrm{MHz}\right) \delta 26.7$; HRMS (ESI) calcd for $\mathrm{C}_{12} \mathrm{H}_{18} \mathrm{BO}_{3}[\mathrm{M}+\mathrm{H}]^{+} \mathrm{m} / \mathrm{z} 221.1349$, found 221.1350.

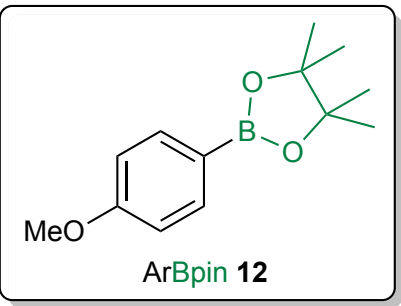

2-(4-Methoxyphenyl)-4,4,5,5-tetramethyl-1,3,2-dioxaborolane (12). Followed Method $A$ using aroyl fluoride ${ }^{7} 10$ and $B_{2}$ (pin) $)_{2}$. Purification by flash chromatography on silica gel (hexanes/EtOAc, 95:5) afforded 12 as a white solid (44 mg, 63\% yield): $\mathrm{mp} 27-28{ }^{\circ} \mathrm{C} ;{ }^{1} \mathrm{H} \mathrm{NMR}\left(\mathrm{CDCl}_{3}, 400 \mathrm{MHz}\right) \delta$ $7.75(\mathrm{~d}, J=8.6 \mathrm{~Hz}, 2 \mathrm{H}), 6.90(\mathrm{~d}, \mathrm{~J}=8.6 \mathrm{~Hz}, 2 \mathrm{H}), 3.83(\mathrm{~s}, 3 \mathrm{H}), 1.33(\mathrm{~s}, 12 \mathrm{H}) ;{ }^{13} \mathrm{C} \mathrm{NMR}\left(\mathrm{CDCl}_{3}\right.$, $126 \mathrm{MHz}) \delta 162.36,136.72,113.52,83.76,55.31,25.08$, the carbon attached to boron was not observed due to quadrupolar coupling; ${ }^{11} \mathrm{~B} \mathrm{NMR}\left(\mathrm{CDCl}_{3}, 128 \mathrm{MHz}\right) \delta 30.7$; HRMS (ESI) calcd for $\mathrm{C}_{13} \mathrm{H}_{20} \mathrm{BO}_{3}[\mathrm{M}+\mathrm{H}]^{+} \mathrm{m} / \mathrm{z} 235.1506$, found 243.1506.

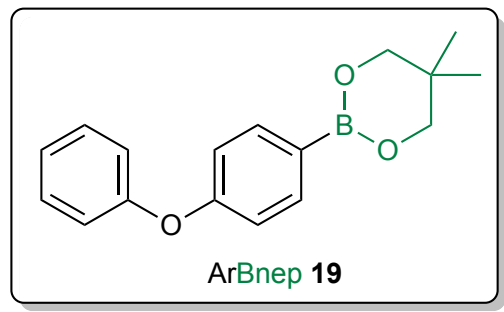

5,5-Dimethyl-2-(4-phenoxyphenyl)-1,3,2-dioxaborinane (19). Followed Method A using 4phenoxybenzoyl fluoride ${ }^{8}$ and $\mathrm{B}_{2}(\mathrm{nep})_{2}$. Purification by flash chromatography on silica gel (hexanes/EtOAc, 95:5) afforded 19 as a white solid (65 mg, 77\% yield): $\mathrm{mp} 61-62{ }^{\circ} \mathrm{C}$; ${ }^{1} \mathrm{H}$ NMR $\left(700 \mathrm{MHz}, \mathrm{CDCl}_{3}\right) \delta 7.79(\mathrm{~d}, J=8.4 \mathrm{~Hz}, 2 \mathrm{H}), 7.35(\mathrm{dd}, J=8.5,7.4 \mathrm{~Hz}, 2 \mathrm{H}), 7.13(\mathrm{~m}, 1 \mathrm{H}), 7.04(\mathrm{~d}$, $J=7.4 \mathrm{~Hz}, 2 \mathrm{H}), 6.99(\mathrm{~d}, J=8.4 \mathrm{~Hz}, 2 \mathrm{H}), 3.77(\mathrm{~s}, 4 \mathrm{H}), 1.04(\mathrm{~s}, 6 \mathrm{H}) ;{ }^{13} \mathrm{C} \mathrm{NMR}\left(176 \mathrm{MHz}, \mathrm{CDCl}_{3}\right) \delta$ 
$159.88,156.99,135.85,129.94,123.66,119.53,117.85,72.50,32.10,22.12$, the carbon attached to boron was not observed due to quadrupolar coupling; ${ }^{11} \mathrm{~B}$ NMR $\left(\mathrm{CDCl}_{3}, 128 \mathrm{MHz}\right) \delta 26.7$; HRMS (ESI) calcd for $\mathrm{C}_{17} \mathrm{H}_{20} \mathrm{BO}_{3}[\mathrm{M}+\mathrm{H}]^{+} \mathrm{m} / \mathrm{z} 283.1506$, found 283.1506 .

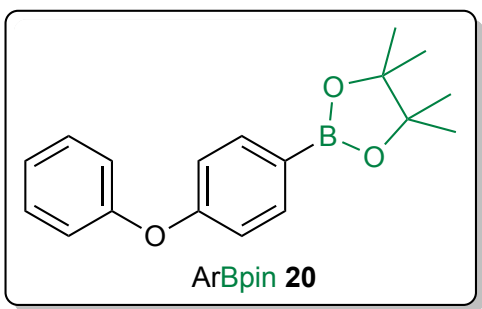

4,4,5,5-Tetramethyl-2-(4-phenoxyphenyl)-1,3,2-dioxaborolane (20). Followed Method A using 4-phenoxybenzoyl fluoride ${ }^{8}$ and $\mathrm{B}_{2}(\text { pin })_{2}$. Purification by flash chromatography on silica gel (hexanes/EtOAc, 95:5) afforded 20 as a colorless oil (58 mg, 65\% yield): ${ }^{1} \mathrm{H}$ NMR $\left(\mathrm{CDCl}_{3}, 500\right.$ $\mathrm{MHz}) \delta 7.78(\mathrm{~d}, J=8.5 \mathrm{~Hz}, 2 \mathrm{H}), 7.36-7.34(\mathrm{~m}, 2 \mathrm{H}), 7.12(\mathrm{t}, J=7.9 \mathrm{~Hz}, 1 \mathrm{H}), 7.03(\mathrm{~d}, J=8.5 \mathrm{~Hz}$, $2 \mathrm{H}), 6.98(\mathrm{~d}, J=8.5 \mathrm{~Hz}, 2 \mathrm{H}), 1.34(\mathrm{~s}, 12 \mathrm{H}) ;{ }^{13} \mathrm{C} \mathrm{NMR}\left(\mathrm{CDCl}_{3}, 176 \mathrm{MHz}\right) \delta 160.39,156.77,136.84$, $130.00,123.86,119.67,117.89,83.95,25.08$, the carbon attached to boron was not observed due to quadrupolar coupling; ${ }^{11} \mathrm{~B}$ NMR $\left(\mathrm{CDCl}_{3}, 128 \mathrm{MHz}\right) \delta 29.6$; HRMS (ESI) calcd for $\mathrm{C}_{18} \mathrm{H}_{22} \mathrm{BO}_{3}$ $[\mathrm{M}+\mathrm{H}]^{+} \mathrm{m} / \mathrm{z} 297.1662$, found 279.1662 .

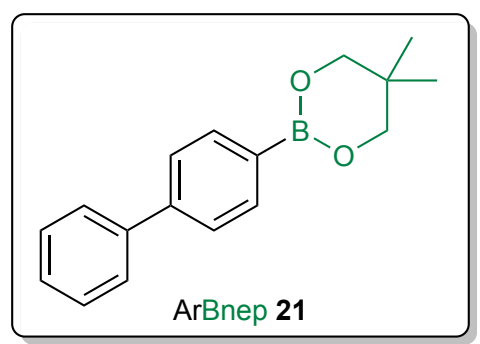

2-([1,1'-Biphenyl]-4-yl)-5,5-dimethyl-1,3,2-dioxaborinane (21). Followed Method B using 4biphenylbenzoic acid and $\mathrm{B}_{2}(\text { nep })_{2}$. Purification by flash chromatography on silica gel (hexanes/EtOAc, 95:5) afforded 21 as a white solid (40 mg, 50\% yield): $\mathrm{mp} 139-141^{\circ} \mathrm{C}$; ${ }^{1} \mathrm{H}$ NMR $\left(700 \mathrm{MHz}, \mathrm{CDCl}_{3}\right) \delta 7.88(\mathrm{~d}, \mathrm{~J}=7.9 \mathrm{~Hz}, 2 \mathrm{H}$ ), 7.67-7.51 (multiple peaks, 4H), $7.42(\mathrm{~m}, 2 \mathrm{H}), 7.36$ $(\mathrm{m}, 1 \mathrm{H}), 3.80(\mathrm{~s}, 4 \mathrm{H}), 1.05(\mathrm{~s}, 6 \mathrm{H}) ;{ }^{13} \mathrm{C}$ NMR $\left(176 \mathrm{MHz}, \mathrm{CDCl}_{3}\right) \delta$ 143.49, 141.42, 134.55, 128.93, $127.60,127.42,126.55,72.56,32.14,22.14$, the carbon attached to boron was not observed due to quadrupolar coupling; ${ }^{11} \mathrm{~B}$ NMR $\left(\mathrm{CDCl}_{3}, 128 \mathrm{MHz}\right) \delta 26.8$; HRMS (ESI) calcd for $\mathrm{C}_{17} \mathrm{H}_{20} \mathrm{BO}_{2}$ $[\mathrm{M}+\mathrm{H}]^{+} \mathrm{m} / \mathrm{z} 267.1556$, found 267.1553 . 


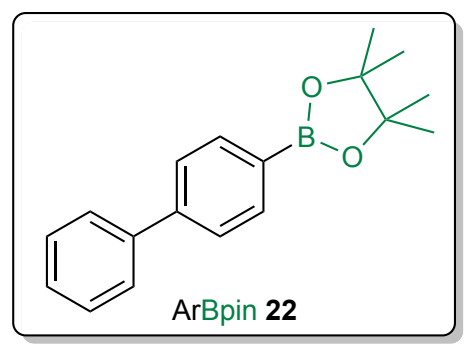

2-([1,1'-Biphenyl]-4-yl)-4,4,5,5-tetramethyl-1,3,2-dioxaborolane (22). Followed Method B using 4-biphenylbenzoic acid and $\mathrm{B}_{2}(\text { pin })_{2}$. Purification by flash chromatography on silica gel (hexanes/EtOAc, 96:4) afforded 22 as a white solid (58 mg, 69\% yield): $\mathrm{mp} \mathrm{111-112}{ }^{\circ} \mathrm{C}$; ${ }^{1} \mathrm{H}$ NMR $\left(\mathrm{CDCl}_{3}, 700 \mathrm{MHz}\right) \delta 7.89$ (d, J=7.5 Hz, 2H), 7.64-7.55 (multiple peaks, 4H), 7.45 (t, $\mathrm{J}=7.5 \mathrm{~Hz}$, $2 \mathrm{H}), 7.35(\mathrm{~m}, 1 \mathrm{H}), 1.37(\mathrm{~s}, 12 \mathrm{H}) ;{ }^{13} \mathrm{C} \mathrm{NMR}\left(\mathrm{CDCl}_{3}, 126 \mathrm{MHz}\right) \delta 144.09,141.22,135.46,128.96$, $127.75,127.43,126.66,84.01,25.10$, the carbon attached to boron was not observed due to quadrupolar coupling; ${ }^{11} \mathrm{~B}$ NMR $\left(\mathrm{CDCl}_{3}, 128 \mathrm{MHz}\right) \delta 30.5$; HRMS (ESI) calcd for $\mathrm{C}_{18} \mathrm{H}_{22} \mathrm{BO}_{2}$ $[\mathrm{M}+\mathrm{H}]^{+} \mathrm{m} / \mathrm{z} 281.1713$, found 281.1712 .

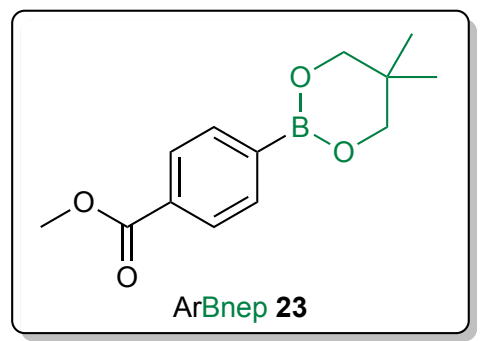

Methyl 4-(5,5-dimethyl-1,3,2-dioxaborinan-2-yl)benzoate (23). Followed Method B using 4(methoxycarbonyl)benzoic acid and $\mathrm{B}_{2}(\mathrm{nep})_{2}$. Purification by flash chromatography on silica gel (hexanes/EtOAc, 90:10) afforded 23 as a white solid (55 mg, 74\% yield): $\mathrm{mp} 112-113{ }^{\circ} \mathrm{C} ;{ }^{1} \mathrm{H}$ NMR $\left(700 \mathrm{MHz}, \mathrm{CDCl}_{3}\right) \delta 8.00(\mathrm{~d}, \mathrm{~J}=8.0 \mathrm{~Hz}, 2 \mathrm{H}), 7.86(\mathrm{~d}, \mathrm{~J}=8.0 \mathrm{~Hz}, 2 \mathrm{H}), 3.91(\mathrm{~s}, 3 \mathrm{H}), 3.78(\mathrm{~s}, 4 \mathrm{H})$, 1.03 (s, 6H); ${ }^{13} \mathrm{C}$ NMR $\left(176 \mathrm{MHz}, \mathrm{CDCl}_{3}\right) \delta 167.53,133.96,132.01,128.70,72.59,52.28,32.10$, 22.10, the carbon attached to boron was not observed due to quadrupolar coupling; ${ }^{11} \mathrm{~B}$ NMR $\left(\mathrm{CDCl}_{3}, 128 \mathrm{MHz}\right) \delta 26.9$; HRMS $(\mathrm{ESI})$ calcd for $\mathrm{C}_{13} \mathrm{H}_{18} \mathrm{BO}_{4}[\mathrm{M}+\mathrm{H}]^{+} \mathrm{m} / \mathrm{z} 249.1298$, found 249.1299.

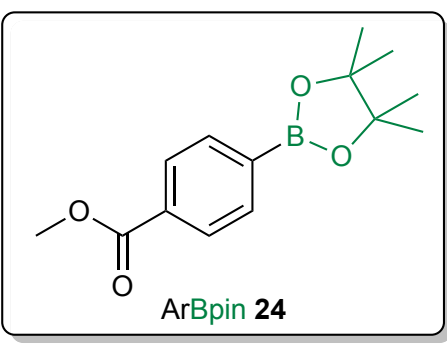

Methyl 4-(4,4,5,5-tetramethyl-1,3,2-dioxaborolan-2-yl)benzoate (24). Followed Method B using 4-(methoxycarbonyl)benzoic acid and $\mathrm{B}_{2}(\text { pin })_{2}$. Purification by flash chromatography on silica gel (hexanes/EtOAc, 90:10) afforded 24 as a white solid (61 mg, 77\% yield): $\mathrm{mp} 77-78{ }^{\circ} \mathrm{C}$; 
${ }^{1} \mathrm{H} \mathrm{NMR}\left(\mathrm{CDCl}_{3}, 700 \mathrm{MHz}\right) \delta 8.02(\mathrm{~d}, J=8.2 \mathrm{~Hz}, 2 \mathrm{H}), 7.87(\mathrm{~d}, J=8.2 \mathrm{~Hz}, 2 \mathrm{H}), 3.92(\mathrm{~s}, 3 \mathrm{H}), 1.35$ (s, 12H); ${ }^{13} \mathrm{C} \mathrm{NMR}\left(\mathrm{CDCl}_{3}, 126 \mathrm{MHz}\right) \delta 167.27,134.83,132.48,128.76,84.34,52.30,25.06$, the carbon attached to boron was not observed due to quadrupolar coupling; ${ }^{11} \mathrm{~B}$ NMR $\left(\mathrm{CDCl}_{3}, 128\right.$ $\mathrm{MHz}) \delta 30.3$; HRMS (ESI) calcd for $\mathrm{C}_{14} \mathrm{H}_{20} \mathrm{BO}_{4}[\mathrm{M}+\mathrm{H}]^{+} \mathrm{m} / \mathrm{z} 263.1455$, found 263.1020.

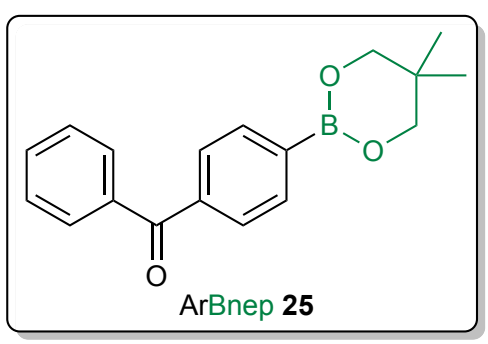

(4-(5,5-Dimethyl-1,3,2-dioxaborinan-2-yl)phenyl)(phenyl)methanone (25). Followed Method $\mathrm{B}$ using 4-benzoylbenzoic acid and $\mathrm{B}_{2}(\mathrm{nep})_{2}$. Purification by flash chromatography on silica gel (hexanes/EtOAc, 95:5) afforded 25 as a white solid (50 mg, 57\% yield): $\mathrm{mp} 138-140{ }^{\circ} \mathrm{C} ;{ }^{1} \mathrm{H}$ NMR $\left(700 \mathrm{MHz}, \mathrm{CDCl}_{3}\right) \delta 7.91(\mathrm{~d}, J=7.3 \mathrm{~Hz}, 2 \mathrm{H}), 7.80(\mathrm{~d}, J=8.1 \mathrm{~Hz}, 2 \mathrm{H}), 7.75(\mathrm{~m}, 2 \mathrm{H}), 7.58(\mathrm{~m}, 1 \mathrm{H})$, $7.48(\mathrm{~m}, 2 \mathrm{H}), 3.80(\mathrm{~s}, 4 \mathrm{H}), 1.05(\mathrm{~s}, 6 \mathrm{H}) ;{ }^{13} \mathrm{C}$ NMR $\left(176 \mathrm{MHz}, \mathrm{CDCl}_{3}\right) \delta 197.29,139.48,137.89$, $133.86,132.61,130.31,129.19,128.45,72.63,32.14,22.12$, the carbon attached to boron was not observed due to quadrupolar coupling; ${ }^{11} \mathrm{~B}$ NMR $\left(\mathrm{CDCl}_{3}, 128 \mathrm{MHz}\right) \delta 26.6$; HRMS (ESI) calcd for $\mathrm{C}_{18} \mathrm{H}_{20} \mathrm{BO}_{3}[\mathrm{M}+\mathrm{H}]^{+} \mathrm{m} / \mathrm{z} 295.1506$, found 295.1508.

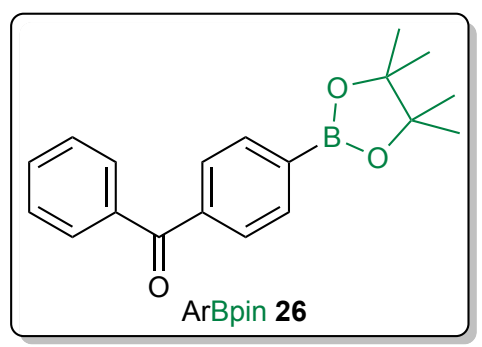

Phenyl(4-(4,4,5,5-tetramethyl-1,3,2-dioxaborolan-2-yl)phenyl)methanone (26). Followed Method $\mathrm{B}$ using 4-benzoylbenzoic acid and $\mathrm{B}_{2}(\text { pin })_{2}$. Purification by flash chromatography on silica gel (hexanes/EtOAc, 95:5) afforded 26 as a white solid (65 mg, 70\% yield): $\mathrm{mp} 110-111^{\circ} \mathrm{C} ;{ }^{1} \mathrm{H}$ NMR $\left(\mathrm{CDCl}_{3}, 700 \mathrm{MHz}\right) \delta 7.91(\mathrm{~m}, 2 \mathrm{H}), 7.81-7.76$ (multiple peaks, $\left.4 \mathrm{H}\right), 7.58(\mathrm{t}, J=7.5 \mathrm{~Hz}, 1 \mathrm{H}$ ), $7.48(\mathrm{~d}, J=8.1 \mathrm{~Hz}, 2 \mathrm{H}), 1.37(\mathrm{~s}, 12 \mathrm{H}) ;{ }^{13} \mathrm{C} \mathrm{NMR}\left(\mathrm{CDCl}_{3}, 126 \mathrm{MHz}\right) \delta 197.13,139.92,137.64$, $134.71,132.69,130.32,128.97,128.42,84.30,25.02$, the carbon attached to boron was not observed due to quadrupolar coupling; ${ }^{11} \mathrm{~B} \mathrm{NMR}\left(\mathrm{CDCl}_{3}, 128 \mathrm{MHz}\right) \delta 30.6$; HRMS (ESI) calcd for $\mathrm{C}_{19} \mathrm{H}_{22} \mathrm{BO}_{3}[\mathrm{M}+\mathrm{H}]^{+} \mathrm{m} / \mathrm{z}$ 309.1662, found 309.1665. 


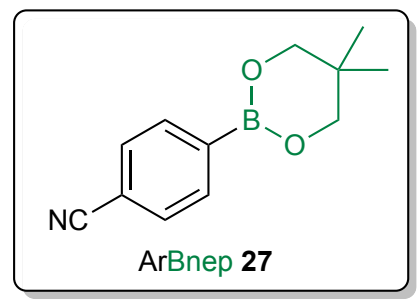

4-(5,5-Dimethyl-1,3,2-dioxaborinan-2-yl)benzonitrile (27). Followed Method A using 4cyanobenzoyl fluoride and $\mathrm{B}_{2}(\text { nep })_{2}$. Purification by flash chromatography on silica gel (hexanes/EtOAc, 95:5) afforded 27 as a white solid (53 mg, 82\% yield): mp 113-115 ${ }^{\circ} \mathrm{C}$; ${ }^{1} \mathrm{H}$ NMR $\left(700 \mathrm{MHz}, \mathrm{CDCl}_{3}\right) \delta 7.87(\mathrm{~d}, J=7.7 \mathrm{~Hz}, 2 \mathrm{H}), 7.62$ (d, J = $\left.7.7 \mathrm{~Hz}, 2 \mathrm{H}\right), 3.78(\mathrm{~s}, 4 \mathrm{H}), 1.03(\mathrm{~s}, 6 \mathrm{H})$; ${ }^{13} \mathrm{C}$ NMR $\left(176 \mathrm{MHz}, \mathrm{CDCl}_{3}\right) \delta 134.45,131.25,119.34,114.16,72.65,32.14,22.07$, the carbon attached to boron was not observed due to quadrupolar coupling; ${ }^{11} \mathrm{~B}$ NMR $\left(\mathrm{CDCl}_{3}, 128 \mathrm{MHz}\right)$ $\delta$ 26.7; HRMS (ESI) calcd for $\mathrm{C}_{12} \mathrm{H}_{15} \mathrm{BNO}_{2}[\mathrm{M}+\mathrm{H}]^{+} \mathrm{m} / \mathrm{z} 216.1196$, found 216.1197.

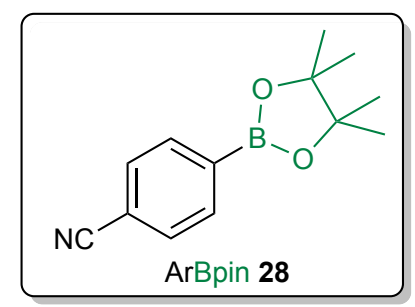

4-(4,4,5,5-Tetramethyl-1,3,2-dioxaborolan-2-yl)benzonitrile (28). Followed Method A using 4cyanobenzoyl fluoride and $\mathrm{B}_{2}$ (pin) $)_{2}$. Purification by flash chromatography on silica gel (hexanes/EtOAc, 95:5) afforded 28 as a white solid (45 mg, 66\% yield): $\mathrm{mp} \mathrm{100-101}{ }^{\circ} \mathrm{C}$; ${ }^{1} \mathrm{H}$ NMR $\left(\mathrm{CDCl}_{3}, 700 \mathrm{MHz}\right) \delta 7.88(\mathrm{~d}, J=8.2 \mathrm{~Hz}, 2 \mathrm{H}), 7.64(\mathrm{~d}, J=8.2 \mathrm{~Hz}, 2 \mathrm{H}), 1.35(\mathrm{~s}, 12 \mathrm{H}) ;{ }^{13} \mathrm{C}$ NMR $\left(\mathrm{CDCl}_{3}, 126 \mathrm{MHz}\right) \delta 135.28,131.30,119.03,114.72,84.68,25.05$, the carbon attached to boron was not observed due to quadrupolar coupling; ${ }^{11} \mathrm{~B}$ NMR $\left(\mathrm{CDCl}_{3}, 128 \mathrm{MHz}\right) \delta 30.4$; HRMS (ESI) calcd for $\mathrm{C}_{13} \mathrm{H}_{17} \mathrm{BNO}_{2}[\mathrm{M}+\mathrm{H}]^{+} \mathrm{m} / \mathrm{z} 230.1352$, found 230.1350 .

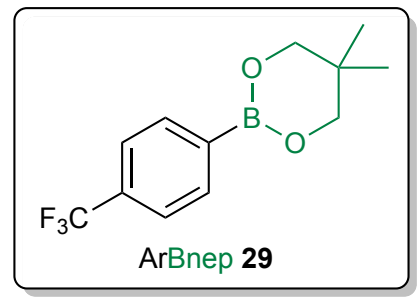

5,5-Dimethyl-2-(4-(trifluoromethyl)phenyl)-1,3,2-dioxaborinane (29). Followed Method A using 4-(trifluoromethyl)benzoyl fluoride and $\mathrm{B}_{2}(\text { nep) })_{2}$. Ni(cod) $)_{2} / \mathrm{PCy}_{3}(10 / 20 \mathrm{~mol} \%$ ) were used as the catalyst system instead of catalyst $\boldsymbol{B}$. Purification by flash chromatography on silica gel (hexanes/EtOAc, 97:3) afforded 29 as a white solid (60 mg, 78\% yield): $\mathrm{mp} 108-110{ }^{\circ} \mathrm{C} ;{ }^{1} \mathrm{H}$ NMR $\left(700 \mathrm{MHz}, \mathrm{CDCl}_{3}\right) \delta 7.91(\mathrm{~d}, J=7.7 \mathrm{~Hz}, 2 \mathrm{H}), 7.60$ (d, J = $\left.7.7 \mathrm{~Hz}, 2 \mathrm{H}\right), 3.79(\mathrm{~s}, 4 \mathrm{H}), 1.03(\mathrm{~s}, 6 \mathrm{H})$; ${ }^{13} \mathrm{C}$ NMR (176 MHz, $\left.\mathrm{CDCl}_{3}\right) \delta$ 134.32, 132.48 (q, $\left.J=31.9 \mathrm{~Hz}\right), 124.52$ (q, J = 272.2 Hz), 124.38 (q, $J=3.7 \mathrm{~Hz}), 72.61,32.12,22.07$, the carbon attached to boron was not observed due to 
quadrupolar coupling; ${ }^{11} \mathrm{~B} \mathrm{NMR}\left(\mathrm{CDCl}_{3}, 128 \mathrm{MHz}\right) \delta 26.9 ;{ }^{19} \mathrm{~F} \mathrm{NMR}\left(\mathrm{CDCl}_{3}, 375 \mathrm{MHz}\right) \delta-62.5(\mathrm{~s}$, $3 F)$; HRMS (ESI) calcd for $\mathrm{C}_{12} \mathrm{H}_{15} \mathrm{BF}_{3} \mathrm{O}_{2}[\mathrm{M}+\mathrm{H}]^{+} \mathrm{m} / \mathrm{z} 259.1117$, found 259.1120.

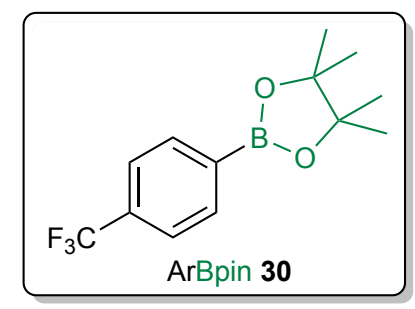

4,4,5,5-Tetramethyl-2-(4-(trifluoromethyl)phenyl)-1,3,2-dioxaborolane (30). Followed Method A using 4-(trifluoromethyl)benzoyl fluoride and $\mathrm{B}_{2}(\mathrm{pin})_{2}$. Ni(cod) $)_{2} / \mathrm{PCy}_{3}(10 / 20 \mathrm{~mol} \%)$ was used as the catalyst instead of $\boldsymbol{B}$. Purification by flash chromatography on silica gel (hexanes/EtOAc, 97:3) afforded 30 as a white solid $\left(57 \mathrm{mg}, 70 \%\right.$ yield): $\mathrm{mp} 73-75^{\circ} \mathrm{C} ;{ }^{1} \mathrm{H} \mathrm{NMR}\left(\mathrm{CDCl}_{3}, 700 \mathrm{MHz}\right) \delta$ $7.91(\mathrm{~d}, J=7.6 \mathrm{~Hz}, 2 \mathrm{H}), 7.62$ (d, $J=7.6 \mathrm{~Hz}, 2 \mathrm{H}), 1.35$ (s, 12H); ${ }^{13} \mathrm{C} \mathrm{NMR}\left(\mathrm{CDCl}_{3}, 126 \mathrm{MHz}\right)$ $\delta 135.02,132.91$ (q, $J=32.0 \mathrm{~Hz}$ ), 125.54 (q, $J=271.0 \mathrm{~Hz}$ ), 124.32 (q, $J=7.0 \mathrm{~Hz}$ ), 84.24, 24.83, the carbon attached to boron was not observed due to quadrupolar coupling; ${ }^{11} \mathrm{~B} \mathrm{NMR}\left(\mathrm{CDCl}_{3}\right.$, $128 \mathrm{MHz}) \delta 30.6 ;{ }^{19} \mathrm{~F} \mathrm{NMR}\left(\mathrm{CDCl}_{3}, 375 \mathrm{MHz}\right) \delta-63.2$ (s, 3F); HRMS (ESI) calcd for $\mathrm{C}_{13} \mathrm{H}_{17} \mathrm{BF}_{3} \mathrm{O}_{2}$ $[\mathrm{M}+\mathrm{H}]^{+} \mathrm{m} / \mathrm{z} 273.1274$, found 273.1275 .

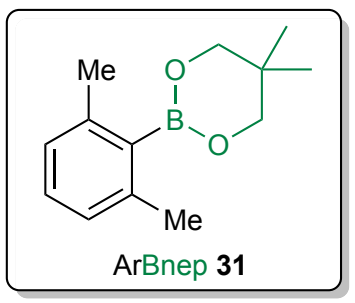

2-(2,6-Dimethylphenyl)-5,5-dimethyl-1,3,2-dioxaborinane (31). Followed Method $B$ using 2,6dimethylbenzoic acid and $\mathrm{B}_{2}(\mathrm{nep})_{2}$. Ni(cod) $)_{2} / \mathrm{PCy}_{3}(10 / 20 \mathrm{~mol} \%)$ were used as the catalyst system instead of catalyst $\boldsymbol{B}$. Purification by flash chromatography on silica gel (hexanes/EtOAc, 98:2) afforded 31 as a white solid (38 mg, 58\% yield): mp 132-134 ${ }^{\circ} \mathrm{C} ;{ }^{1} \mathrm{H}$ NMR $\left(700 \mathrm{MHz}, \mathrm{CDCl}_{3}\right) \delta 7.10$ $(\mathrm{t}, J=7.6 \mathrm{~Hz}, 1 \mathrm{H}), 6.94(\mathrm{~d}, J=7.6 \mathrm{~Hz}, 2 \mathrm{H}), 3.80(\mathrm{~s}, 4 \mathrm{H}), 2.39(\mathrm{~s}, 6 \mathrm{H}), 1.11(\mathrm{~s}, 6 \mathrm{H}) ;{ }^{13} \mathrm{C}$ NMR $(176$ $\left.\mathrm{MHz}_{\mathrm{CDCl}}\right) \delta 140.64,128.65,126.54,72.46,31.86,22.48,22.47$, the carbon attached to boron was not observed due to quadrupolar coupling; ${ }^{11} \mathrm{~B}$ NMR $\left(\mathrm{CDCl}_{3}, 128 \mathrm{MHz}\right) \delta 28.1$; HRMS (ESI) calcd for $\mathrm{C}_{13} \mathrm{H}_{20} \mathrm{BO}_{2}[\mathrm{M}+\mathrm{H}]^{+} \mathrm{m} / \mathrm{z} 219.1556$, found 219.1558 .

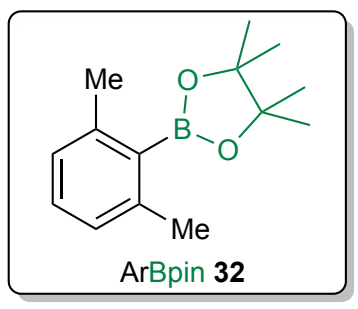

2-(2,6-Dimethylphenyl)-4,4,5,5-tetramethyl-1,3,2-dioxaborolane (32). Followed Method B 
using 2,6-dimethylbenzoic acid and $\mathrm{B}_{2}(\mathrm{pin})_{2}$. Ni(cod) $)_{2} / \mathrm{PCy}_{3}(10 / 20 \mathrm{~mol} \%)$ was used as the catalyst instead of $\boldsymbol{B}$. Purification by flash chromatography on silica gel (hexanes/EtOAc, 98:2) afforded 32 as a white solid (38 mg, $55 \%$ yield): $\mathrm{mp} 100-101{ }^{\circ} \mathrm{C} ;{ }^{1} \mathrm{H} \mathrm{NMR}\left(\mathrm{CDCl}_{3}, 700 \mathrm{MHz}\right) \delta$ $7.14(\mathrm{t}, J=7.6,1 \mathrm{H}), 6.96(\mathrm{~d}, J=7.6 \mathrm{~Hz}, 2 \mathrm{H}), 2.42(\mathrm{~s}, 6 \mathrm{H}), 1.41(\mathrm{~s}, 12 \mathrm{H}) ;{ }^{13} \mathrm{C} \mathrm{NMR}\left(\mathrm{CDCl}_{3}, 126\right.$ $\mathrm{MHz}) \delta 141.94,129.35,126.61,83.83,25.17,22.42$, the carbon attached to boron was not observed due to quadrupolar coupling; ${ }^{11} \mathrm{~B}$ NMR $\left(\mathrm{CDCl}_{3}, 128 \mathrm{MHz}\right) \delta 32.2$; HRMS (ESI) calcd for $\mathrm{C}_{14} \mathrm{H}_{22} \mathrm{BO}_{2}[\mathrm{M}+\mathrm{H}]^{+} \mathrm{m} / \mathrm{z}$ 233.1713, found 233.1713.

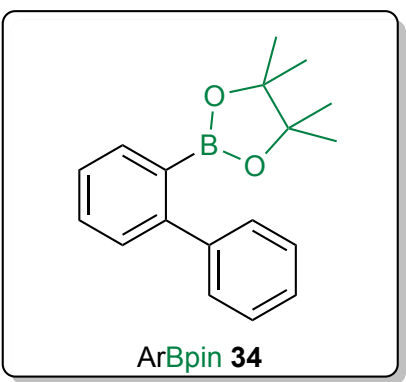

2-([1,1'-Biphenyl]-2-yl)-4,4,5,5-tetramethyl-1,3,2-dioxaborolane (34). Followed Method A using [1,1'-biphenyl]-2-carbonyl fluoride ${ }^{7}$ and $\mathrm{B}_{2}(\text { pin })_{2}$. Purification by flash chromatography on silica gel (hexanes/EtOAc, 98:2) afforded 34 as a white solid (36 mg, $43 \%$ yield): $\mathrm{mp} 80-82{ }^{\circ} \mathrm{C}$; ${ }^{1} \mathrm{H}$ NMR $\left(\mathrm{CDCl}_{3}, 700 \mathrm{MHz}\right) \delta 7.71(\mathrm{~d}, \mathrm{~J}=7.3 \mathrm{~Hz}, 1 \mathrm{H}$ ), 7.30-7.46 (multiple peaks, 8H), 1.20 (s, $12 \mathrm{H}) ;{ }^{13} \mathrm{C} \mathrm{NMR}\left(\mathrm{CDCl}_{3}, 126 \mathrm{MHz}\right) \delta 147.51,143.22,134.45,129.97,129.12,128.92,127.72$, $126.80,125.89,83.74,24.68$, the carbon attached to boron was not observed due to quadrupolar coupling; ${ }^{11} \mathrm{~B}$ NMR $\left(\mathrm{CDCl}_{3}, 128 \mathrm{MHz}\right) \delta 30.2$; HRMS (ESI) calcd for $\mathrm{C}_{14} \mathrm{H}_{22} \mathrm{BO}_{2}[\mathrm{M}+\mathrm{H}]^{+} \mathrm{m} / \mathrm{z}$ 281.1713, found 281.1715 .

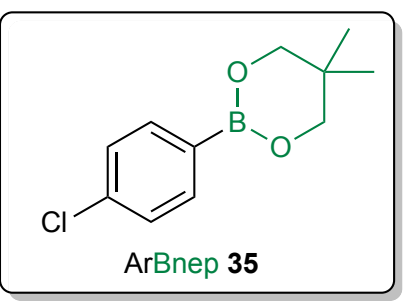

2-(4-Chlorophenyl)-5,5-dimethyl-1,3,2-dioxaborinane (35). Followed Method A using 4chlorobenzoyl fluoride ${ }^{2}$ and $\mathrm{B}_{2}(\mathrm{nep})_{2}$. Purification by flash chromatography on silica gel (hexanes/EtOAc, 95:5) afforded 35 as a white solid (30 mg, $45 \%$ yield): $\mathrm{mp} 72-73{ }^{\circ} \mathrm{C}$; ${ }^{1} \mathrm{H}$ NMR $\left(700 \mathrm{MHz}, \mathrm{CDCl}_{3}\right) \delta 7.72(\mathrm{~d}, J=8.3 \mathrm{~Hz}, 2 \mathrm{H}), 7.32(\mathrm{~d}, J=8.3 \mathrm{~Hz}, 2 \mathrm{H}), 3.76(\mathrm{~s}, 4 \mathrm{H}), 1.02(\mathrm{~s}, 6 \mathrm{H})$; ${ }^{13} \mathrm{C} \mathrm{NMR}\left(176 \mathrm{MHz}, \mathrm{CDCl}_{3}\right) \delta 137.07,135.45,128.03,72.52,32.10,22.10$, the carbon attached to boron was not observed due to quadrupolar coupling; ${ }^{11} \mathrm{~B}$ NMR $\left(\mathrm{CDCl}_{3}, 128 \mathrm{MHz}\right) \delta 26.7$; HRMS (ESI) calcd for $\mathrm{C}_{11} \mathrm{H}_{15} \mathrm{BClO}_{2}[\mathrm{M}+\mathrm{H}]^{+} \mathrm{m} / \mathrm{z} 225.0854$, found 225.0856 . 


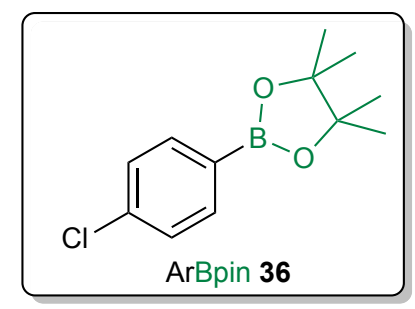

2-(4-Chlorophenyl)-4,4,5,5-tetramethyl-1,3,2-dioxaborolane (36). Followed Method A using 4chlorobenzoyl fluoride ${ }^{2}$ and $\mathrm{B}_{2}$ (pin) $)_{2}$. Purification by flash chromatography on silica gel (hexanes/EtOAc, 95:5) afforded $\mathbf{3 6}$ as a pale yellow liquid (37 mg, 52\% yield): ${ }^{1} \mathrm{H}$ NMR $\left(\mathrm{CDCl}_{3}\right.$, $700 \mathrm{MHz}) \delta 7.73(\mathrm{~d}, \mathrm{~J}=7.4 \mathrm{~Hz}, 2 \mathrm{H}), 7.34(\mathrm{~d}, \mathrm{~J}=7.4 \mathrm{~Hz}, 2 \mathrm{H}), 1.34(\mathrm{~s}, 12 \mathrm{H}) ;{ }^{13} \mathrm{C} \mathrm{NMR}\left(\mathrm{CDCl}_{3}, 126\right.$ $\mathrm{MHz}) \delta 137.74,136.33,128.21,84.22,25.08$, the carbon attached to boron was not observed due to quadrupolar coupling; ${ }^{11} \mathrm{~B}$ NMR $\left(\mathrm{CDCl}_{3}, 128 \mathrm{MHz}\right) \delta 30.7$; HRMS (ESI) calcd for $\mathrm{C}_{12} \mathrm{H}_{17} \mathrm{BCIO}_{2}$ $[\mathrm{M}+\mathrm{H}]^{+} \mathrm{m} / \mathrm{z} 239.1010$, found 239.1012.

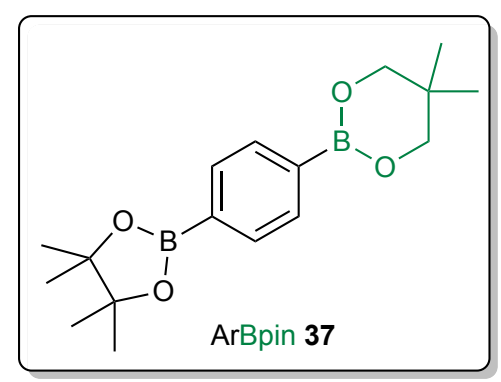

\section{5,5-Dimethyl-2-(4-(4,4,5,5-tetramethyl-1,3,2-dioxaborolan-2-yl)phenyl)-1,3,2-dioxaborinane}

(37). Followed Method B using 4-(4,4,5,5-tetramethyl-1,3,2-dioxaborolan-2-yl)benzoic acid and $\mathrm{B}_{2}$ (pin)2. Purification by flash chromatography on silica gel (hexanes/EtOAc, 90:10) afforded 37

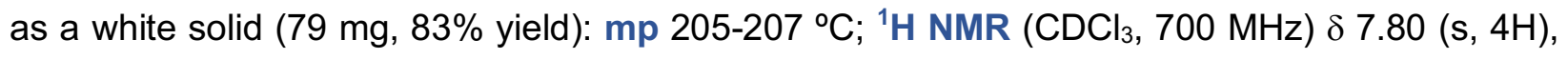

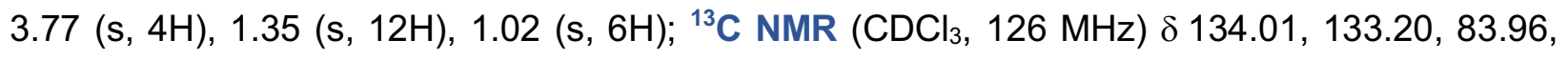
$72.51,32.09,25.08,22.12$, the carbons attached to boron were not observed due to quadrupolar coupling; ${ }^{11} \mathrm{~B}$ NMR $\left(\mathrm{CDCl}_{3}, 128 \mathrm{MHz}\right) \delta 31.0,26.9$; HRMS (ESI) calcd for $\mathrm{C}_{17} \mathrm{H}_{27} \mathrm{~B}_{2} \mathrm{O}_{4}[\mathrm{M}+\mathrm{H}]^{+} \mathrm{m} / \mathrm{z}$ 317.2095, found 317.2098.

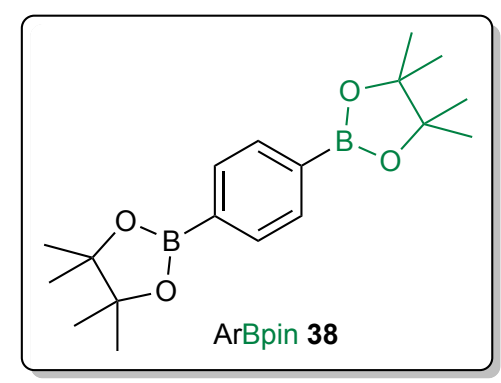

1,4-Bis(4,4,5,5-tetramethyl-1,3,2-dioxaborolan-2-yl)benzene (38). Followed Method B using 4(4,4,5,5-tetramethyl-1,3,2-dioxaborolan-2-yl)benzoic acid and $\mathrm{B}_{2}(\text { pin) })_{2}$. Purification by flash 
chromatography on silica gel (hexanes/EtOAc, 98:2) afforded $\mathbf{3 8}$ as a white solid $(79 \mathrm{mg}, 80 \%$ yield): $\mathrm{mp} 194-196{ }^{\circ} \mathrm{C} ;{ }^{1} \mathrm{H} \mathrm{NMR}\left(\mathrm{CDCl}_{3}, 700 \mathrm{MHz}\right) \delta 7.81$ (s, 4H), 1.35 (s, $\left.24 \mathrm{H}\right) ;{ }^{13} \mathrm{C} \mathrm{NMR}\left(\mathrm{CDCl}_{3}\right.$, $126 \mathrm{MHz}) \delta 134.08,84.04,25.08$, the carbon attached to boron was not observed due to quadrupolar coupling; ${ }^{11} \mathrm{~B}$ NMR $\left(\mathrm{CDCl}_{3}, 128 \mathrm{MHz}\right) \delta 30.7$; HRMS (ESI) calcd for $\mathrm{C}_{18} \mathrm{H}_{29} \mathrm{~B}_{2} \mathrm{O}_{4}$ $[\mathrm{M}+\mathrm{H}]^{+} \mathrm{m} / \mathrm{z} 331.2252$, found 331.2254 .

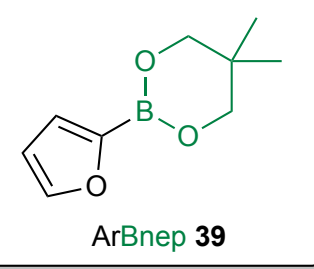

2-(Furan-2-yl)-5,5-dimethyl-1,3,2-dioxaborinane (39). Followed Method B using furan-2carboxylic acid and $\mathrm{B}_{2}(\mathrm{nep})_{2}$. Ni(cod) $)_{2} / \mathrm{PCy}_{3}(10 / 20 \mathrm{~mol} \%)$ were used as the catalyst system instead of catalyst $\boldsymbol{B}$. Purification by flash chromatography on silica gel (hexanes/EtOAc, 95:5) afforded 39 as a white solid ( $41 \mathrm{mg}, 75 \%$ yield): $\mathrm{mp} 86-87^{\circ} \mathrm{C}$; ${ }^{1} \mathrm{H}$ NMR $\left(400 \mathrm{MHz}, \mathrm{CDCl}_{3}\right) \delta 7.62$ (dd, $J=1.7,0.7 \mathrm{~Hz}, 1 \mathrm{H}), 6.98(\mathrm{dd}, J=3.3,0.7 \mathrm{~Hz}, 1 \mathrm{H}), 6.42(\mathrm{dd}, J=3.3,1.7 \mathrm{~Hz}, 1 \mathrm{H}), 3.76(\mathrm{~s}$, $4 \mathrm{H}), 1.03$ (s, 6H); ${ }^{13} \mathrm{C} \mathrm{NMR}\left(126 \mathrm{MHz}, \mathrm{CDCl}_{3}\right) \delta 146.81,121.66,110.37,72.48,32.29,22.07$, the carbon attached to boron was not observed due to quadrupolar coupling; ${ }^{11} \mathrm{~B}$ NMR $\left(\mathrm{CDCl}_{3}, 128\right.$ $\mathrm{MHz}) \delta$ 23.5; HRMS (ESI) calcd for $\mathrm{C}_{9} \mathrm{H}_{14} \mathrm{BO}_{3}[\mathrm{M}+\mathrm{H}]^{+} \mathrm{m} / \mathrm{z}$ 181.1036, found 181.1036.

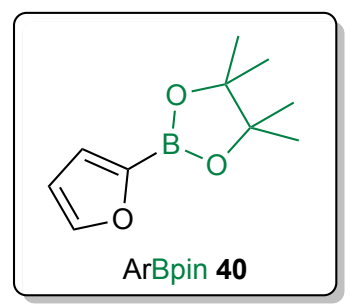

2-(Furan-2-yl)-4,4,5,5-tetramethyl-1,3,2-dioxaborolane (40). Followed Method B using furan-2carboxylic acid and $\mathrm{B}_{2}(\mathrm{pin})_{2}$. Ni(cod) $)_{2} / \mathrm{PCy}_{3}(10 / 20 \mathrm{~mol} \%)$ were used as the catalyst system instead of catalyst $\boldsymbol{B}$. Purification by flash chromatography on silica gel (hexanes/EtOAc, 95:5) afforded 40 as a colorless oil $\left(42 \mathrm{mg}, 72 \%\right.$ yield): ${ }^{1} \mathrm{H}$ NMR $\left(\mathrm{CDCl}_{3}, 700 \mathrm{MHz}\right) \delta 7.63(\mathrm{~d}, J=1.9$ $\mathrm{Hz}, 1 \mathrm{H}), 7.06(\mathrm{~d}, J=2.3 \mathrm{~Hz}, 1 \mathrm{H}), 6.42(\mathrm{~m}, 1 \mathrm{H}), 1.33(\mathrm{~s}, 12 \mathrm{H}) ;{ }^{13} \mathrm{C} \mathrm{NMR}\left(\mathrm{CDCl}_{3}, 176 \mathrm{MHz}\right) \delta 147.48$, $123.38,110.49,84.38,24.93$, the carbon attached to boron was not observed due to quadrupolar coupling; ${ }^{11} \mathrm{~B}$ NMR $\left(\mathrm{CDCl}_{3}, 128 \mathrm{MHz}\right) \delta 27.1$; HRMS (ESI) calcd for $\mathrm{C}_{14} \mathrm{H}_{22} \mathrm{BO}_{2}[\mathrm{M}+\mathrm{H}]^{+} \mathrm{m} / \mathrm{z}$ 195.1192, found 195.1190 .

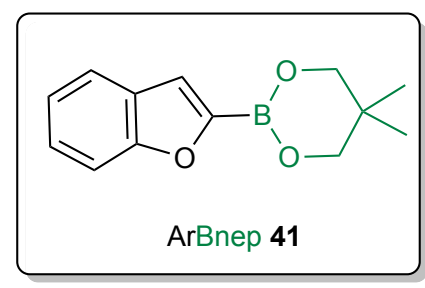


2-(Benzofuran-2-yl)-5,5-dimethyl-1,3,2-dioxaborinane (41). Followed Method A using benzofuran-2-carbonyl fluoride and $\mathrm{B}_{2}(\text { nep })_{2}$. Purification by flash chromatography on silica gel (hexanes/EtOAc, 90:10) afforded 41 as a white solid (54 mg, $78 \%$ yield): $\mathrm{mp} 114-116{ }^{\circ} \mathrm{C} ;{ }^{1} \mathrm{H}$ NMR $\left(700 \mathrm{MHz}, \mathrm{CDCl}_{3}\right) \delta 7.91(\mathrm{~m}, 1 \mathrm{H}), 7.85(\mathrm{~m}, 1 \mathrm{H}), 7.83(\mathrm{~s}, 1 \mathrm{H}), 7.38-7.32$ (multiple peaks, $\left.2 \mathrm{H}\right), 3.81$ (s, 4H), $1.06(\mathrm{~s}, 6 \mathrm{H}) ;{ }^{13} \mathrm{C}$ NMR $\left(176 \mathrm{MHz}, \mathrm{CDCl}_{3}\right) \delta 143.59,140.86,133.00,125.13,124.39$, $124.13,122.71,72.69,32.27,22.10$, the carbon attached to boron was not observed due to quadrupolar coupling; ${ }^{11} \mathrm{~B}$ NMR $\left(\mathrm{CDCl}_{3}, 128 \mathrm{MHz}\right) \delta 25.2$; HRMS (ESI) calcd for $\mathrm{C}_{13} \mathrm{H}_{16} \mathrm{BO}_{3}$ $[\mathrm{M}+\mathrm{H}]^{+} \mathrm{m} / \mathrm{z} 231.1192$, found 231.1192 .

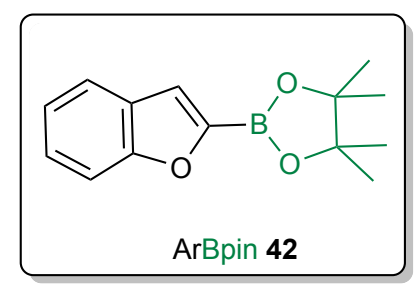

2-(Benzofuran-2-yl)-4,4,5,5-tetramethyl-1,3,2-dioxaborolane (42). Followed Method A using benzofuran-2-carbonyl fluoride and $\mathrm{B}_{2}(\text { pin })_{2}$. Purification by flash chromatography on silica gel (hexanes/EtOAc, 90:10) afforded 42 as a pale yellow solid $\left(40 \mathrm{mg}, 55 \%\right.$ yield): $\mathrm{mp} 83-84{ }^{\circ} \mathrm{C} ;{ }^{1} \mathrm{H}$ NMR $\left(\mathrm{CDCl}_{3}, 700 \mathrm{MHz}\right) \delta 7.63(\mathrm{~d}, J=7.8 \mathrm{~Hz}, 1 \mathrm{H}), 7.57(\mathrm{~d}, J=8.4 \mathrm{~Hz}, 1 \mathrm{H}), 7.40(\mathrm{~d}, J=1.0 \mathrm{~Hz}$, $1 \mathrm{H}), 7.34$ (ddd, $J=8.4,7.1,1.3 \mathrm{~Hz}, 1 \mathrm{H}$ ), 7.23 (ddd, $J=7.8,7.1,1.0 \mathrm{~Hz}, 1 \mathrm{H}), 1.39(\mathrm{~s}, 12 \mathrm{H}) ;{ }^{13} \mathrm{C}$ NMR $\left(\mathrm{CDCl}_{3}, 176 \mathrm{MHz}\right) \delta 157.71,127.68,126.12,122.91,122.07,119.74,112.15,84.87,24.98$, the carbon attached to boron was not observed due to quadrupolar coupling; ${ }^{11} \mathrm{~B} \mathrm{NMR}\left(\mathrm{CDCl}_{3}\right.$, $128 \mathrm{MHz}) \delta$ 28.0; HRMS (ESI) calcd for $\mathrm{C}_{14} \mathrm{H}_{18} \mathrm{BO}_{3}[\mathrm{M}+\mathrm{H}]^{+} \mathrm{m} / \mathrm{z} 245.1349$, found 245.1351 .

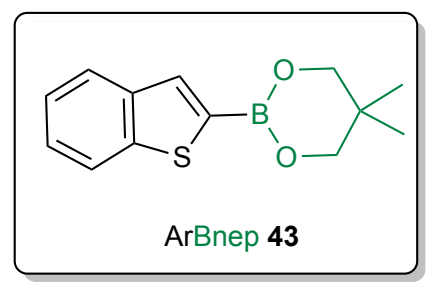

2-(Benzo[b]thiophen-2-yl)-5,5-dimethyl-1,3,2-dioxaborinane (43). Followed Method A using benzo[b]thiophene-2-carbonyl fluoride ${ }^{9}$ and $\mathrm{B}_{2}(\text { nep })_{2}$. Purification by flash chromatography on silica gel (hexanes/EtOAc, 90:10) afforded 43 as a white solid (57 mg, 77\% yield): mp 134-136 ${ }^{\circ} \mathrm{C} ;{ }^{1} \mathrm{H}$ NMR $\left(700 \mathrm{MHz}, \mathrm{CDCl}_{3}\right) \delta 7.62(\mathrm{~d}, J=7.7 \mathrm{~Hz}, 1 \mathrm{H}), 7.56(\mathrm{~d}, J=8.5 \mathrm{~Hz}, 1 \mathrm{H}), 7.37-7.30$ (multiple peaks, 2H), 7.22 (t, J = 7.4 Hz, 1H), $3.83(\mathrm{~s}, 4 \mathrm{H}), 1.06(\mathrm{~s}, 6 \mathrm{H}) ;{ }^{13} \mathrm{C} \mathrm{NMR}\left(\mathrm{CDCl}_{3}, 176\right.$ $\mathrm{MHz}) \delta 157.51,127.96,125.72,122.77,121.94,118.04,112.05,72.70,32.37,22.11$, the carbon attached to boron was not observed due to quadrupolar coupling; ${ }^{11} \mathrm{~B} \mathrm{NMR}\left(\mathrm{CDCl}_{3}, 128 \mathrm{MHz}\right)$ $\delta$ 23.8; HRMS (ESI) calcd for $\mathrm{C}_{13} \mathrm{H}_{16} \mathrm{BO}_{2} \mathrm{~S}[\mathrm{M}+\mathrm{H}]^{+} \mathrm{m} / \mathrm{z} 247.0964$, found 247.0964. 


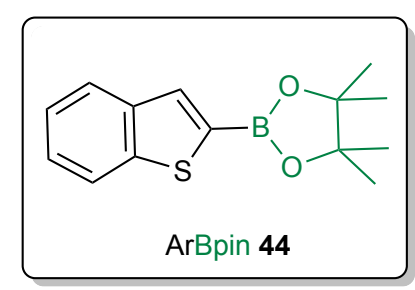

2-(Benzo[b]thiophen-2-yl)-4,4,5,5-tetramethyl-1,3,2-dioxaborolane (44). Followed Method A using benzo[b]thiophene-2-carbonyl fluoride ${ }^{9}$ and $\mathrm{B}_{2}$ (pin) $)_{2}$. Purification by flash chromatography on silica gel (hexanes/EtOAc, 90:10) afforded 44 as a white solid (55 mg, 70\% yield): $\mathrm{mp}$ 73-74 ${ }^{\circ} \mathrm{C}$; ${ }^{1} \mathrm{H}$ NMR $\left(\mathrm{CDCl}_{3}, 700 \mathrm{MHz}\right) \delta$ 7.94-7.90 (multiple peaks, $\left.2 \mathrm{H}\right), 7.87(\mathrm{~d}, \mathrm{~J}=8.0 \mathrm{~Hz}, 1 \mathrm{H}), 7.39$ 7.35 (multiple peaks, 2H), 1.39 (s, 12H); ${ }^{13} \mathrm{C} \mathrm{NMR}\left(\mathrm{CDCl}_{3}, 176 \mathrm{MHz}\right) \delta 143.90,140.62,134.67$, $125.49,124.55,124.29,122.71,84.61,25.00$, the carbon attached to boron was not observed due to quadrupolar coupling; ${ }^{11} \mathrm{~B} \mathrm{NMR}\left(\mathrm{CDCl}_{3}, 128 \mathrm{MHz}\right) \delta 27.9$; HRMS (ESI) calcd for $\mathrm{C}_{14} \mathrm{H}_{18} \mathrm{BO}_{2} \mathrm{~S}[\mathrm{M}+\mathrm{H}]^{+} \mathrm{m} / \mathrm{z} 261.1121$, found 261.1120 .

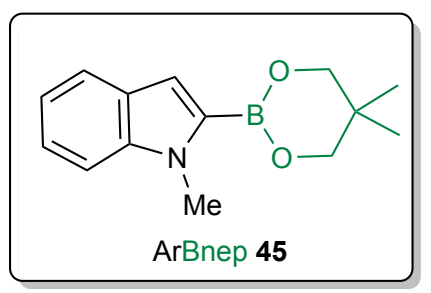

(2-(5,5-Dimethyl-1,3,2-dioxaborinan-2-yl)-1-methyl-1H-indole (45). Followed Method A using 1-methyl-1H-indole-2-carbonyl fluoride and $\mathrm{B}_{2}(\mathrm{nep})_{2}$. Purification by flash chromatography on silica gel (hexanes/EtOAc, 90:10) afforded 45 as a white solid (51 mg, 70\% yield): mp 144-146 ${ }^{\circ} \mathrm{C}$; ${ }^{1} \mathrm{H}$ NMR $\left(400 \mathrm{MHz}, \mathrm{CDCl}_{3}\right) \delta$ 7.66-7.62 (m, 1H), $7.34(\mathrm{~d}, \mathrm{~J}=8.3 \mathrm{~Hz}, 1 \mathrm{H}), 7.26-7.21(\mathrm{~m}, 1 \mathrm{H})$, 7.11-7.04 (multiple peaks, $2 \mathrm{H}), 3.96(\mathrm{~s}, 3 \mathrm{H}), 3.80(\mathrm{~s}, 4 \mathrm{H}), 1.05(\mathrm{~s}, 6 \mathrm{H}) ;{ }^{13} \mathrm{C} \mathrm{NMR}\left(\mathrm{CDCl}_{3}, 126\right.$ $\mathrm{MHz}) \delta 140.42,127.98,122.89,121.59,119.28,113.26,109.77,72.47,32.33,32.16,22.14$, the carbon attached to boron was not observed due to quadrupolar coupling; ${ }^{11} \mathrm{~B}$ NMR $\left(\mathrm{CDCl}_{3}, 128\right.$ $\mathrm{MHz}) \delta$ 23.8; HRMS (ESI) calcd for $\mathrm{C}_{14} \mathrm{H}_{19} \mathrm{BNO}_{2}[\mathrm{M}+\mathrm{H}]^{+} \mathrm{m} / \mathrm{z} 244.1509$, found 244.1511.

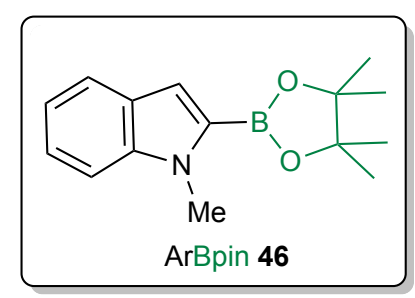

1-Methyl-2-(4,4,5,5-tetramethyl-1,3,2-dioxaborolan-2-yl)-1H-indole (46). Followed Method A using 1-methyl-1H-indole-2-carbonyl fluoride and $\mathrm{B}_{2}(\text { pin) })_{2}$. Purification by flash chromatography on silica gel (hexanes/EtOAc, 90:10) afforded $\mathbf{4 6}$ as a white solid (50 mg, 65\% yield): $\mathrm{mp}$ 98-100 ${ }^{\circ} \mathrm{C} ;{ }^{1} \mathrm{H} \mathrm{NMR}\left(\mathrm{CDCl}_{3}, 700 \mathrm{MHz}\right) \delta 7.67(\mathrm{~d}, J=7.9 \mathrm{~Hz}, 1 \mathrm{H}), 7.37(\mathrm{~m}, 1 \mathrm{H}), 7.29$ (ddd, J = 8.2, 6.9, $1.2 \mathrm{~Hz}, 1 \mathrm{H}), 7.17(\mathrm{~s}, 1 \mathrm{H}), 7.11(\mathrm{ddd}, J=7.9,6.9,1.0 \mathrm{~Hz}, 1 \mathrm{H}), 4.00(\mathrm{~s}, 3 \mathrm{H}), 1.39(\mathrm{~s}, 12 \mathrm{H}) ;{ }^{13} \mathrm{C}$ NMR $\left(\mathrm{CDCl}_{3}, 126 \mathrm{MHz}\right) \delta 140.35,128.06,123.36,121.78,119.48,114.46,109.87,83.88,32.42,25.04$, 
the carbon attached to boron was not observed due to quadrupolar coupling; ${ }^{11} \mathrm{~B} \mathrm{NMR}\left(\mathrm{CDCl}_{3}\right.$, $128 \mathrm{MHz}$ ) $\delta$ 26.6; HRMS (ESI) calcd for $\mathrm{C}_{15} \mathrm{H}_{21} \mathrm{BNO}_{2}[\mathrm{M}+\mathrm{H}]^{+} \mathrm{m} / \mathrm{z} 258.1665$, found 258.1668.

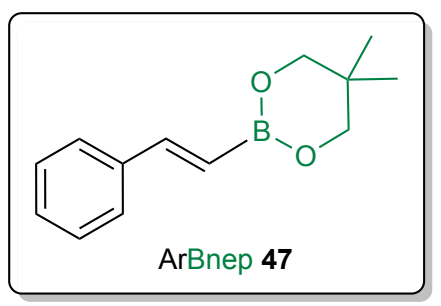

(E)-5,5-dimethyl-2-styryl-1,3,2-dioxaborinane (47). Followed Method A using (E)-cinnamoyl fluoride ${ }^{9}$ and $\mathrm{B}_{2}(\text { nep })_{2}$. Purification by flash chromatography on silica gel (hexanes/EtOAc, 98:2) afforded 47 as a white solid (51 mg, 46\% yield): mp 42-44 ${ }^{\circ} \mathrm{C} ;{ }^{1} \mathrm{H}$ NMR (500 MHz, $\left.\mathrm{CDCl}_{3}\right) \delta 7.50$ (d, J = 7.6 Hz, 2H), 7.36-7.32 (multiple peaks, 3H), $7.28(\mathrm{~m}, 1 \mathrm{H}), 6.12(\mathrm{~d}, J=18.3 \mathrm{~Hz}, 1 \mathrm{H}), 3.71$ $(\mathrm{s}, 4 \mathrm{H}), 1.01(\mathrm{~s}, 6 \mathrm{H}) ;{ }^{13} \mathrm{C} \mathrm{NMR}\left(\mathrm{CDCl}_{3}, 126 \mathrm{MHz}\right) \delta 147.31,137.98,128.71,128.70,127.18,72.39$, $32.05,22.07$, the carbon attached to boron was not observed due to quadrupolar coupling; ${ }^{11} \mathrm{~B}$ NMR $\left(\mathrm{CDCl}_{3}, 128 \mathrm{MHz}\right) \delta 26.8$; HRMS (ESI) calcd for $\mathrm{C}_{13} \mathrm{H}_{18} \mathrm{BO}_{2}[\mathrm{M}+\mathrm{H}]^{+} \mathrm{m} / \mathrm{z} 217.1400$, found 217.1401.

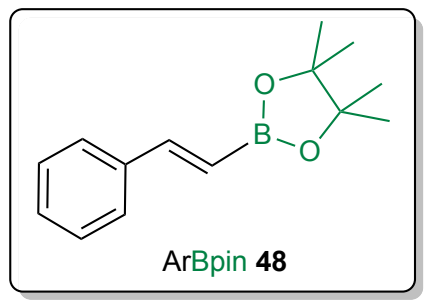

(E)-4,4,5,5-tetramethyl-2-styryl-1,3,2-dioxaborolane (48). Followed Method A using (E)cinnamoyl fluoride ${ }^{9}$ and $\mathrm{B}_{2}$ (pin) $)_{2}$. Purification by flash chromatography on silica gel (hexanes/EtOAc, 98:2) afforded 48 as a pale yellow solid $\left(50 \mathrm{mg}, 32 \%\right.$ yield): $\mathrm{mp} 30-32{ }^{\circ} \mathrm{C} ;{ }^{1} \mathrm{H}$ $\operatorname{NMR}\left(\mathrm{CDCl}_{3}, 500 \mathrm{MHz}\right) \delta 7.49(\mathrm{~d}, J=7.5 \mathrm{~Hz}, 2 \mathrm{H}), 7.41(\mathrm{~d}, J=18.4 \mathrm{~Hz}, 1 \mathrm{H}), 7.35-7.28$ (multiple peaks, $3 \mathrm{H}), 6.18(\mathrm{~d}, \mathrm{~J}=18.4 \mathrm{~Hz}, 1 \mathrm{H}), 1.32(\mathrm{~s}, 12 \mathrm{H}) ;{ }^{13} \mathrm{C} \mathrm{NMR}\left(\mathrm{CDCl}_{3}, 126 \mathrm{MHz}\right) \delta 149.71,137.68$, $129.09,128.77,127.26,83.55,25.03$, the carbon attached to boron was not observed due to quadrupolar coupling; ${ }^{11} \mathrm{~B}$ NMR $\left(\mathrm{CDCl}_{3}, 128 \mathrm{MHz}\right) \delta 30.0$; HRMS (ESI) calcd for $\mathrm{C}_{14} \mathrm{H}_{20} \mathrm{BO}_{2}$ $[\mathrm{M}+\mathrm{H}]^{+} \mathrm{m} / \mathrm{z} 231.1556$, found 231.1556.

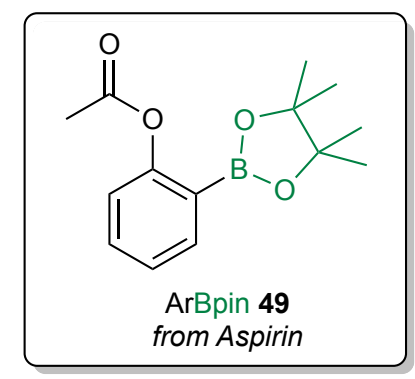

2-(4,4,5,5-Tetramethyl-1,3,2-dioxaborolan-2-yl)phenyl acetate (49). Followed Method A using 
aspirin acid fluoride 49 -COF and $\mathrm{B}_{2}$ (pin) $)_{2}$. Purification by flash chromatography on silica gel (hexanes/EtOAc, 90:10) afforded 49 as a colorless oil (46 mg, 58\% yield): ${ }^{1} \mathrm{H}$ NMR $\left(\mathrm{CDCl}_{3}, 500\right.$ $\mathrm{MHz}) \delta 7.82(\mathrm{dd}, J=7.4,1.8 \mathrm{~Hz}, 1 \mathrm{H}), 7.47(\mathrm{td}, J=7.9,1.8 \mathrm{~Hz}, 1 \mathrm{H}), 7.24(\mathrm{td}, J=7.4,1.0 \mathrm{~Hz}, 1 \mathrm{H})$, 7.01 (dd, $J=7.9,1.0 \mathrm{~Hz}, 1 \mathrm{H}), 2.31$ (s, 3H), 1.32 (s, 12H); ${ }^{13} \mathrm{C} \mathrm{NMR}\left(\mathrm{CDCl}_{3}, 176 \mathrm{MHz}\right) \delta 170.70$, $155.91,136.73,132.81,125.70,121.88,83.92,25.04,21.40$, the carbon attached to boron was not observed due to quadrupolar coupling; ${ }^{11} \mathrm{~B}$ NMR $\left(\mathrm{CDCl}_{3}, 128 \mathrm{MHz}\right) \delta 30.2$; HRMS (ESI) calcd for $\mathrm{C}_{14} \mathrm{H}_{20} \mathrm{BO}_{2}[\mathrm{M}+\mathrm{H}]^{+} \mathrm{m} / \mathrm{z} 263.1455$, found 263.1460.

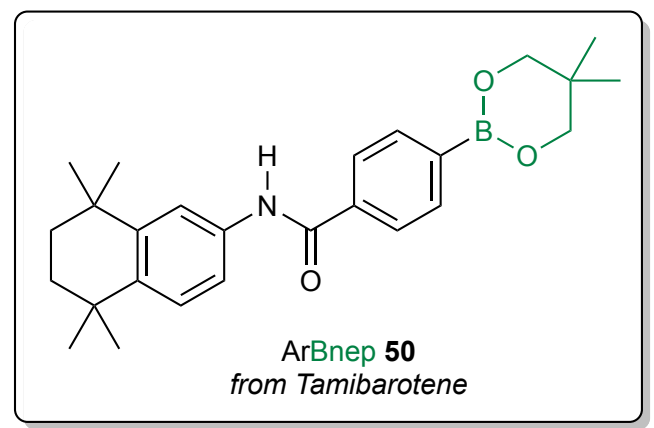

\section{4-(5,5-Dimethyl-1,3,2-dioxaborinan-2-yl)-N-(5,5,8,8-tetramethyl-5,6,7,8-tetrahydronaphtha-} len-2-yl)benzamide (50). Followed Method $B$ using tamibarotene and $B_{2}(n e p)_{2}$. Purification by flash chromatography on silica gel (hexanes/EtOAc, 95:5) afforded $\mathbf{5 0}$ as a white solid (59 mg, $70 \%$ yield): mp 139-141 ${ }^{\circ} \mathrm{C} ;{ }^{1} \mathrm{H}$ NMR $\left(700 \mathrm{MHz}, \mathrm{CDCl}_{3}\right) \delta 8.14(\mathrm{~d}, J=8.0 \mathrm{~Hz}, 2 \mathrm{H}), 7.93$ (d, J = 8.0 $\mathrm{Hz}, 2 \mathrm{H}), 7.81(\mathrm{~s}, 1 \mathrm{H}), 7.54(\mathrm{~s}, 1 \mathrm{H}), 7.44(\mathrm{~d}, J=8.5 \mathrm{~Hz}, 1 \mathrm{H}), 7.32(\mathrm{~d}, J=8.5 \mathrm{~Hz}, 1 \mathrm{H}), 4.22(\mathrm{~s}, 4 \mathrm{H})$, $1.70(\mathrm{~s}, 4 \mathrm{H}), 1.30(\mathrm{~s}, 6 \mathrm{H}), 1.28(\mathrm{~s}, 6 \mathrm{H}), 1.03(\mathrm{~s}, 6 \mathrm{H}) ;{ }^{13} \mathrm{C} \mathrm{NMR}\left(\mathrm{CDCl}_{3}, 176 \mathrm{MHz}\right) \delta 166.40,146.14$, $142.10,139.50,135.18,132.99,130.27,127.56,127.34,118.34,70.36,36.98,35.25,35.21$, $34.68,34.26,32.06,32.03,21.82$, the carbon attached to boron was not observed due to quadrupolar coupling; ${ }^{11} \mathrm{~B}$ NMR $\left(\mathrm{CDCl}_{3}, 128 \mathrm{MHz}\right) \delta 26.9$; HRMS (ESI) calcd for $\mathrm{C}_{26} \mathrm{H}_{35} \mathrm{BNO}_{3}$ $[\mathrm{M}+\mathrm{H}]^{+} \mathrm{m} / \mathrm{z}$ 420.2710, found 420.2715 .

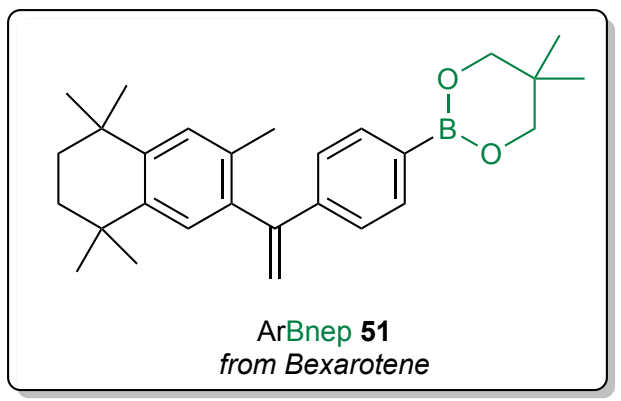

\section{5,5-Dimethyl-2-(4-(1-(3,5,5,8,8-pentamethyl-5,6,7,8-tetrahydronaphthalen-2-yl)vinyl)phen-} yl)-1,3,2-dioxaborinane (51). Followed Method $B$ using bexarotene and $\mathrm{B}_{2}(\mathrm{nep})_{2}$. Purification by flash chromatography on silica gel (hexanes/EtOAc, 95:5) afforded $\mathbf{5 1}$ as a white solid (63 mg, $76 \%$ yield): mp 132-134 ${ }^{\circ} \mathrm{C} ;{ }^{1} \mathrm{H}$ NMR $\left(700 \mathrm{MHz}, \mathrm{CDCl}_{3}\right) \delta 7.73(\mathrm{~d}, \mathrm{~J}=7.0 \mathrm{~Hz}, 2 \mathrm{H}), 7.28$ (d, J = 7.0 $\mathrm{Hz}, 2 \mathrm{H}), 7.14(\mathrm{~s}, 1 \mathrm{H}), 7.07(\mathrm{~s}, 1 \mathrm{H}), 5.77(\mathrm{~s}, 1 \mathrm{H}), 5.23(\mathrm{~s}, 1 \mathrm{H}), 3.77(\mathrm{~s}, 4 \mathrm{H}), 1.97(\mathrm{~s}, 3 \mathrm{H}), 1.70(\mathrm{~s}$, $4 \mathrm{H}), 1.31(\mathrm{~s}, 6 \mathrm{H}), 1.28(\mathrm{~s}, 6 \mathrm{H}), 1.03(\mathrm{~s}, 6 \mathrm{H}) ;{ }^{13} \mathrm{C} \mathrm{NMR}\left(\mathrm{CDCl}_{3}, 176 \mathrm{MHz}\right) \delta 150.18,144.12,143.36$, 
$142.24,138.87,134.03,133.10,128.30,128.05,126.05,115.43,72.52,35.49,35.48,34.19$, $34.09,32.15,32.13,32.11,22.15,20.19$, the carbon attached to boron was not observed due to quadrupolar coupling; ${ }^{11} \mathrm{~B}$ NMR $\left(\mathrm{CDCl}_{3}, 128 \mathrm{MHz}\right) \delta 26.4$; HRMS (ESI) calcd for $\mathrm{C}_{28} \mathrm{H}_{37} \mathrm{BO}_{2}$ $[\mathrm{M}+\mathrm{H}]^{+} \mathrm{m} / \mathrm{z}$ 417.2965, found 417.2965.

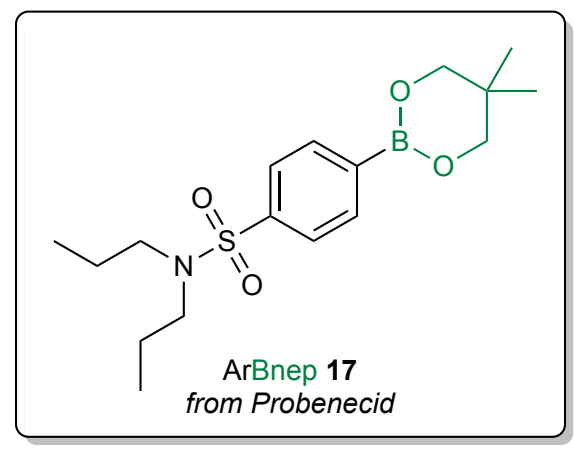

4-(5,5-Dimethyl-1,3,2-dioxaborinan-2-yl)-N,N-dipropylbenzenesulfonamide (17). Followed Method $A$ using probenecid acid fluoride 16 and $B_{2}(\text { nep })_{2}$. Purification by flash chromatography on silica gel (hexanes/EtOAc, 90:10) afforded 17 as a white solid (96 mg, 90\% yield): $\mathrm{mp}$ 185$187^{\circ} \mathrm{C} ;{ }^{1} \mathrm{H}$ NMR $\left(700 \mathrm{MHz}, \mathrm{CDCl}_{3}\right) \delta 7.88(\mathrm{~d}, J=8.4 \mathrm{~Hz}, 2 \mathrm{H}), 7.74(\mathrm{~d}, \mathrm{~J}=8.4 \mathrm{~Hz}, 2 \mathrm{H}), 3.75(\mathrm{~s}$, 4H), $3.04(\mathrm{~m}, 4 \mathrm{H}), 1.49(\mathrm{~m}, 4 \mathrm{H}), 1.00(\mathrm{~s}, 6 \mathrm{H}), 0.83(\mathrm{t}, \mathrm{J}=7.4 \mathrm{~Hz}, 6 \mathrm{H}) ;{ }^{13} \mathrm{C} \mathrm{NMR}\left(\mathrm{CDCl}_{3}, 176 \mathrm{MHz}\right)$ $\delta 141.85,134.46,126.02,72.54,50.11,32.02,22.09,21.98,11.35$, the carbon attached to boron was not observed due to quadrupolar coupling; ${ }^{11} \mathrm{~B}$ NMR $\left(\mathrm{CDCl}_{3}, 128 \mathrm{MHz}\right) \delta 26.5$; HRMS (ESI) calcd for $\mathrm{C}_{17} \mathrm{H}_{29} \mathrm{BNO}_{4} \mathrm{~S}[\mathrm{M}+\mathrm{H}]^{+} \mathrm{m} / \mathrm{z} 354.1910$, found 354.1910.

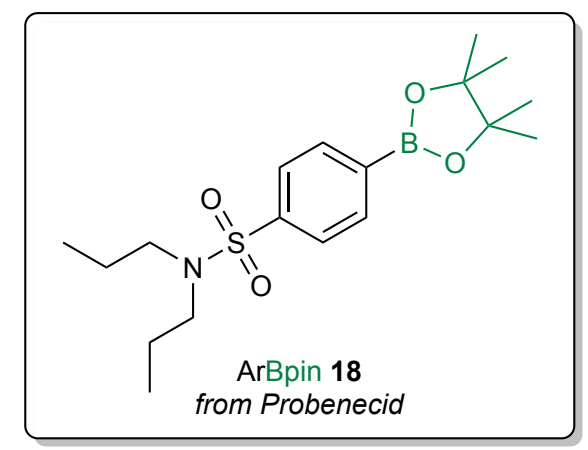

$N, N$-Dipropyl-4-(4,4,5,5-tetramethyl-1,3,2-dioxaborolan-2-yl)benzenesulfonamide

(18). Followed Method A using probenecid acid fluoride 16 and $B_{2}(\operatorname{pin})_{2}$. Purification by flash chromatography on silica gel (hexanes/EtOAc, 90:10) afforded 18 as a white solid (93 mg, 84\% yield): $\mathrm{mp} 149-151^{\circ} \mathrm{C} ;{ }^{1} \mathrm{H} \mathrm{NMR}\left(\mathrm{CDCl}_{3}, 700 \mathrm{MHz}\right) \delta 7.91$ (d, $\left.J=8.3 \mathrm{~Hz}, 2 \mathrm{H}\right), 7.78(\mathrm{~d}, J=8.3 \mathrm{~Hz}$, 2H), $3.05(\mathrm{~m}, 4 \mathrm{H}), 1.52(\mathrm{~m}, 4 \mathrm{H}), 1.35(\mathrm{~s}, 12 \mathrm{H}), 0.85(\mathrm{t}, J=7.4 \mathrm{~Hz}, 6 \mathrm{H}) ;{ }^{13} \mathrm{C} \mathrm{NMR}\left(\mathrm{CDCl}_{3}, 176\right.$ $\mathrm{MHz}) \delta 142.54,135.38,126.19,84.53,50.13,25.20,22.12,11.36$, the carbon attached to boron was not observed due to quadrupolar coupling; ${ }^{11} \mathrm{~B}$ NMR $\left(\mathrm{CDCl}_{3}, 128 \mathrm{MHz}\right) \delta 30.7$; HRMS (ESI) calcd for $\mathrm{C}_{18} \mathrm{H}_{31} \mathrm{BNO}_{4} \mathrm{~S}[\mathrm{M}+\mathrm{H}]^{+} \mathrm{m} / \mathrm{z} 368.2067$, found 368.2070. 


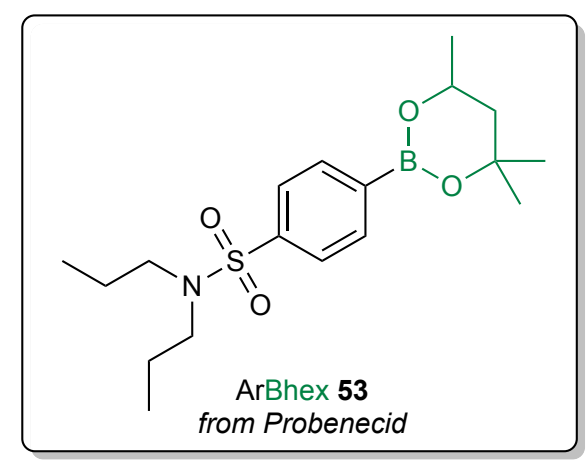

N,N-dipropyl-4-(4,4,6-trimethyl-1,3,2-dioxaborinan-2-yl)benzenesulfonamide (53). Followed Method A using probenecid acid fluoride 16 and $\mathrm{B}_{2}(\mathrm{pin})_{2}$. Purification by flash chromatography on silica gel (hexanes/EtOAc, 90:10) afforded 53 as a white solid (93 mg, 84\% yield): mp 190-192 ${ }^{\circ} \mathrm{C} ;{ }^{1} \mathrm{H}$ NMR $\left(\mathrm{CDCl}_{3}, 500 \mathrm{MHz}\right) \delta 7.90(\mathrm{~d}, J=8.3 \mathrm{~Hz}, 2 \mathrm{H}), 7.79(\mathrm{~d}, J=8.3 \mathrm{~Hz}, 2 \mathrm{H}), 4.39$ (ddq, $J=$ 12.3, 6.2, 3.1 Hz, 1H), $3.05(\mathrm{~m}, 4 \mathrm{H}), 1.90(\mathrm{dd}, J=13.8,3.0 \mathrm{~Hz}, 1 \mathrm{H}), 1.64(\mathrm{dd}, J=13.8,11.5 \mathrm{~Hz}$, $1 \mathrm{H}), 1.50(\mathrm{~m}, 4 \mathrm{H}), 1.46(\mathrm{~s}, 3 \mathrm{H}), 1.44(\mathrm{~s}, 3 \mathrm{H}) 1.42(\mathrm{~d}, J=6.2 \mathrm{~Hz}, 3 \mathrm{H}), 0.86(\mathrm{t}, J=7.4 \mathrm{~Hz}, 6 \mathrm{H}) ;{ }^{13} \mathrm{C}$ NMR $\left(\mathrm{CDCl}_{3}, 126 \mathrm{MHz}\right) \delta 143.81,135.41,126.12,71.54,65.04,50.11,46.12,31.38,28.35$, $23.30,22.15,11.38$, the carbon attached to boron was not observed due to quadrupolar coupling; ${ }^{11} \mathrm{~B}$ NMR $\left(\mathrm{CDCl}_{3}, 128 \mathrm{MHz}\right) \delta 27.2$; HRMS (ESI) calcd for $\mathrm{C}_{18} \mathrm{H}_{31} \mathrm{BNO}_{4} \mathrm{~S}[\mathrm{M}+\mathrm{H}]^{+} \mathrm{m} / \mathrm{z} 368.2067$, found 368.2067.

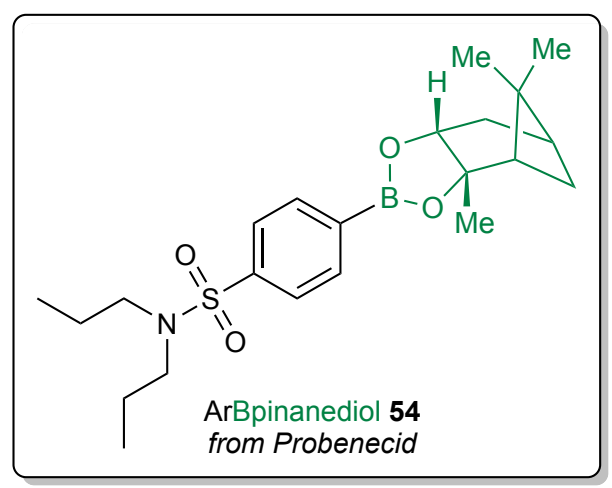

N,N-dipropyl-4-((3aS,7aR)-3a,5,5-trimethylhexahydro-4,6-methanobenzo[d][1,3,2]-dioxaborol-2-yl)benzenesulfonamide (54). Followed Method A using probenecid acid fluoride 16 and $\mathrm{B}_{2}$ (pin)2. Purification by flash chromatography on silica gel (hexanes/EtOAc, 95:5) afforded 54 as a white solid (93 mg, 84\% yield): $\mathrm{mp} 182-184{ }^{\circ} \mathrm{C} ;{ }^{1} \mathrm{H} \mathrm{NMR}\left(\mathrm{CDCl}_{3}, 700 \mathrm{MHz}\right) \delta 7.92$ (d, J = 8.0 $\mathrm{Hz}, 2 \mathrm{H}), 7.78(\mathrm{~d}, J=8.0 \mathrm{~Hz}, 2 \mathrm{H}), 4.47(\mathrm{dd}, J=8.8,1.8 \mathrm{~Hz}, 1 \mathrm{H}), 3.06(\mathrm{~m}, 4 \mathrm{H}), 2.42(\mathrm{~m}, 1 \mathrm{H}), 2.24$ $(\mathrm{m}, 1 \mathrm{H}), 2.15(\mathrm{t}, J=5.5 \mathrm{~Hz}, 0 \mathrm{H}), 1.98-1.90$ (multiple peaks, $2 \mathrm{H}), 1.53(\mathrm{~m}, 4 \mathrm{H}), 1.48(\mathrm{~s}, 3 \mathrm{H}), 1.31$ $(\mathrm{s}, 3 \mathrm{H}), 1.17$ (d, J = $11.0 \mathrm{~Hz}, 1 \mathrm{H}), 0.88(\mathrm{~s}, 3 \mathrm{H}), 0.85$ (t, J = 7.4 Hz, 6H); ${ }^{3} \mathrm{C}$ NMR $\left(\mathrm{CDCl}_{3}, 176\right.$ $\mathrm{MHz}) \delta 142.47,135.45,126.24,87.00,78.76,51.49,50.17,39.65,38.41,35.60,28.84,27.26$, $26.67,24.22,22.16,11.38$, the carbon attached to boron was not observed due to quadrupolar coupling; ${ }^{11} \mathrm{~B}$ NMR $\left(\mathrm{CDCl}_{3}, 128 \mathrm{MHz}\right) \delta 27.1$; HRMS (ESI) calcd for $\mathrm{C}_{18} \mathrm{H}_{31} \mathrm{BNO}_{4} \mathrm{~S}[\mathrm{M}+\mathrm{H}]^{+} \mathrm{m} / \mathrm{z}$ 420.2380, found 420.2381 . 


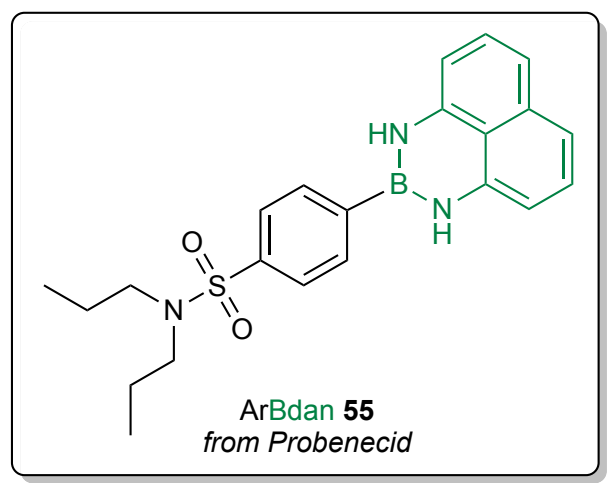

4-(1H-Naphtho[1,8-de][1,3,2]diazaborinin-2(3H)-yl)-N,N-dipropylbenzenesulfonamide (55). Followed Method A using probenecid acid fluoride 16 and $B_{2}(\text { pin })_{2}$. Purification by flash chromatography on silica gel (hexanes/EtOAc, 90:10) afforded 55 as a white solid (93 $\mathrm{mg}, 84 \%$ yield): $\mathrm{mp} 265-267^{\circ} \mathrm{C} ;{ }^{1} \mathrm{H}$ NMR $\left(\mathrm{CDCl}_{3}, 500 \mathrm{MHz}\right) \delta 7.83(\mathrm{~d}, \mathrm{~J}=7.7 \mathrm{~Hz}, 2 \mathrm{H}), 7.74$ (d, $\mathrm{J}=7.8 \mathrm{~Hz}$, 2H), $7.15(\mathrm{t}, J=7.8 \mathrm{~Hz}, 2 \mathrm{H}), 7.08(\mathrm{~d}, J=8.3 \mathrm{~Hz}, 2 \mathrm{H}), 6.44(\mathrm{~d}, J=7.2 \mathrm{~Hz}, 2 \mathrm{H}), 6.07(\mathrm{~s}, 2 \mathrm{H}), 3.11$ $(\mathrm{t}, J=7.7 \mathrm{~Hz}, 4 \mathrm{H}), 1.57(\mathrm{~m}, 4 \mathrm{H}), 0.88(\mathrm{t}, J=7.4 \mathrm{~Hz}, 6 \mathrm{H}) ;{ }^{13} \mathrm{C} \mathrm{NMR}\left(\mathrm{CDCl}_{3}, 126 \mathrm{MHz}\right) \delta 141.72$, $140.84,136.51,132.28,127.84,126.78,120.19,118.44,106.52,50.22,22.22,11.41$, the carbon attached to boron was not observed due to quadrupolar coupling; ${ }^{11} \mathrm{~B} \mathrm{NMR}\left(\mathrm{CDCl}_{3}, 128 \mathrm{MHz}\right)$ $\delta$ 28.9; HRMS (ESI) calcd for $\mathrm{C}_{22} \mathrm{H}_{27} \mathrm{BN}_{3} \mathrm{O}_{2} \mathrm{~S}[\mathrm{M}+\mathrm{H}]^{+} \mathrm{m} / \mathrm{z} 408.1917$, found 408.1920. 


\section{References}

(1) Tolman, C. A. Phosphorus ligand exchange equilibrium on zerovalent nickel. Dominant role for steric effects. J. Am. Chem. Soc. 1970, 92, 2956.

(2) Malapit, C. A.; Bour, J. R.; Brigham, C. E.; Sanford, M. S. Base-free nickel-catalysed decarbonylative Suzuki-Miyaura coupling of acid fluorides. Nature 2018, 563, 100.

(3) $\quad\left[\left(\mathrm{PCy}_{3}\right)_{2} \mathrm{Ni}(\mathrm{Cl})(\mathrm{o}\right.$-tol $\left.)\right]$ was prepared by following the procedure reported by Jamison. See: Standley, E. A.; Smith, S. J.; Müller, P.; Jamison, T. F. A broadly applicable strategy for entry into homogeneous nickel(0) catalysts from air-stable nickel(II) complexes. Organometallics 2014, 33, 2012. We note that the $\mathrm{Ni}-\mathrm{Cl}$ complex is also commercially available (CAS: 123839-28-1).

(4) Ishiyama, T.; Murata, M.; Miyaura, N. Palladium(0)-catalyzed cross-coupling reaction of alkoxydiboron with haloarenes: a direct procedure for arylboronic esters. J. Org. Chem. 1995, 60, 7508.

(5) Carpino, L. A. \& El-Faham, A. Tetramethylfluoroformamidinium hexafluorophosphate: a rapid-acting peptide coupling reagent for solution and solid phase peptide synthesis. A. J. Am. Chem. Soc. 1995, 117, 5401.

(6) Carpino, L. A., Beyermann, M., Wenschuh, H. \& Bienert, M. Peptide synthesis via amino acid halides. Acc. Chem. Res. 1996, 29, 268.

(7) Cismesia, M. A., Ryan, S. J., Bland, D. C. \& Sanford, M. S. J. Org. Chem. 2017, 82, 5020.

(8) Keaveney, S. T. \& Schoenebeck, F. Palladium-catalyzed decarbonylative trifluoromethylation of acid fluorides. Angew. Chem. Int. Ed. 2018, 57, 4073.

(9) Wang, X.; Wang, Z.; Asanuma, Y.; Nishihara, Y. Synthesis of 2-substituted propenes by bidentate phosphine-assisted methylenation of acyl fluorides and acyl chlorides with $\mathrm{AlMe}_{3}$. Org. Lett. 2019, 21, 3640. 
XVI. Copies of ${ }^{1} \mathrm{H},{ }^{13} \mathrm{C},{ }^{19} \mathrm{~F}$ and ${ }^{31} \mathrm{P}$ NMR Spectra

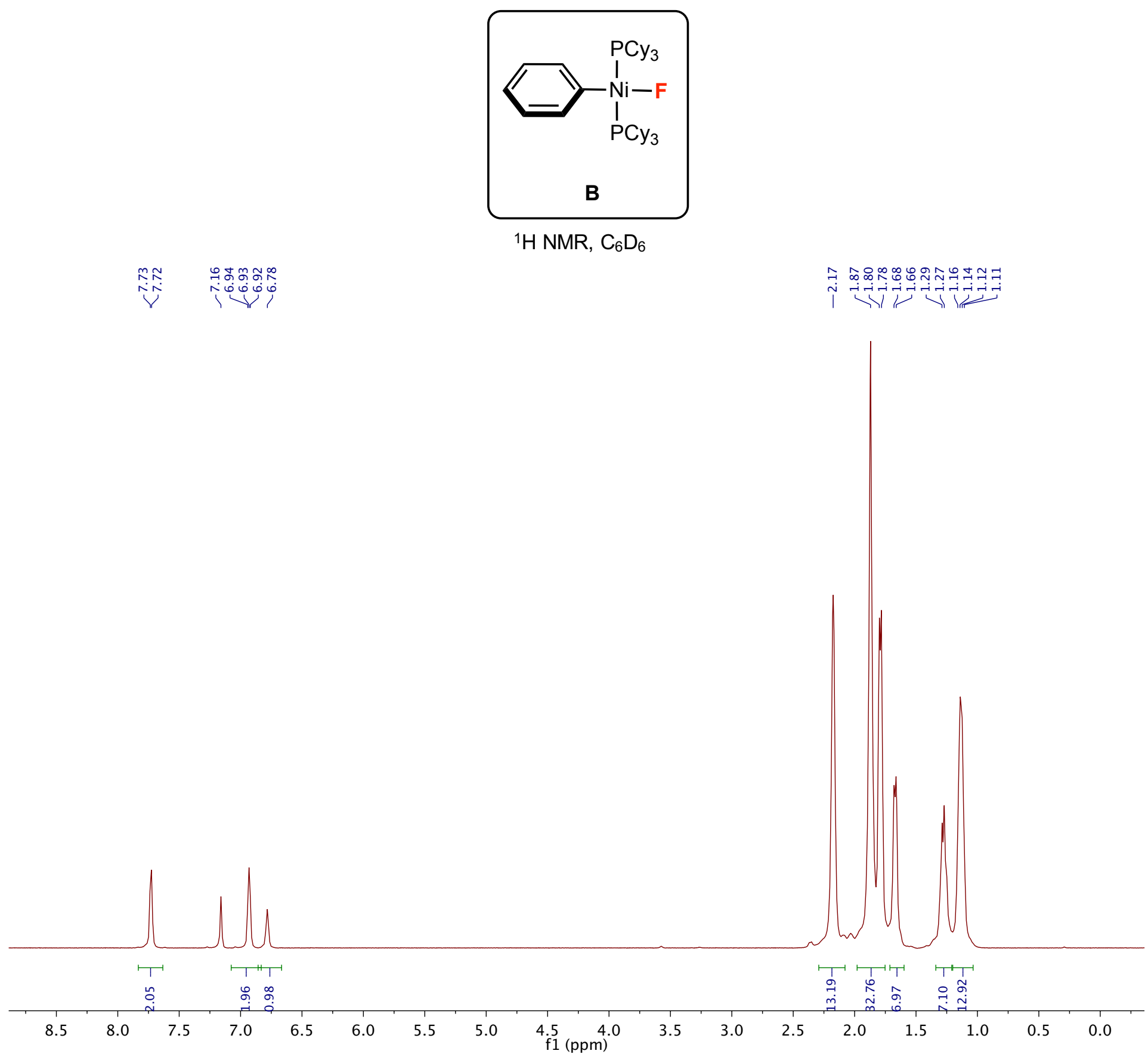



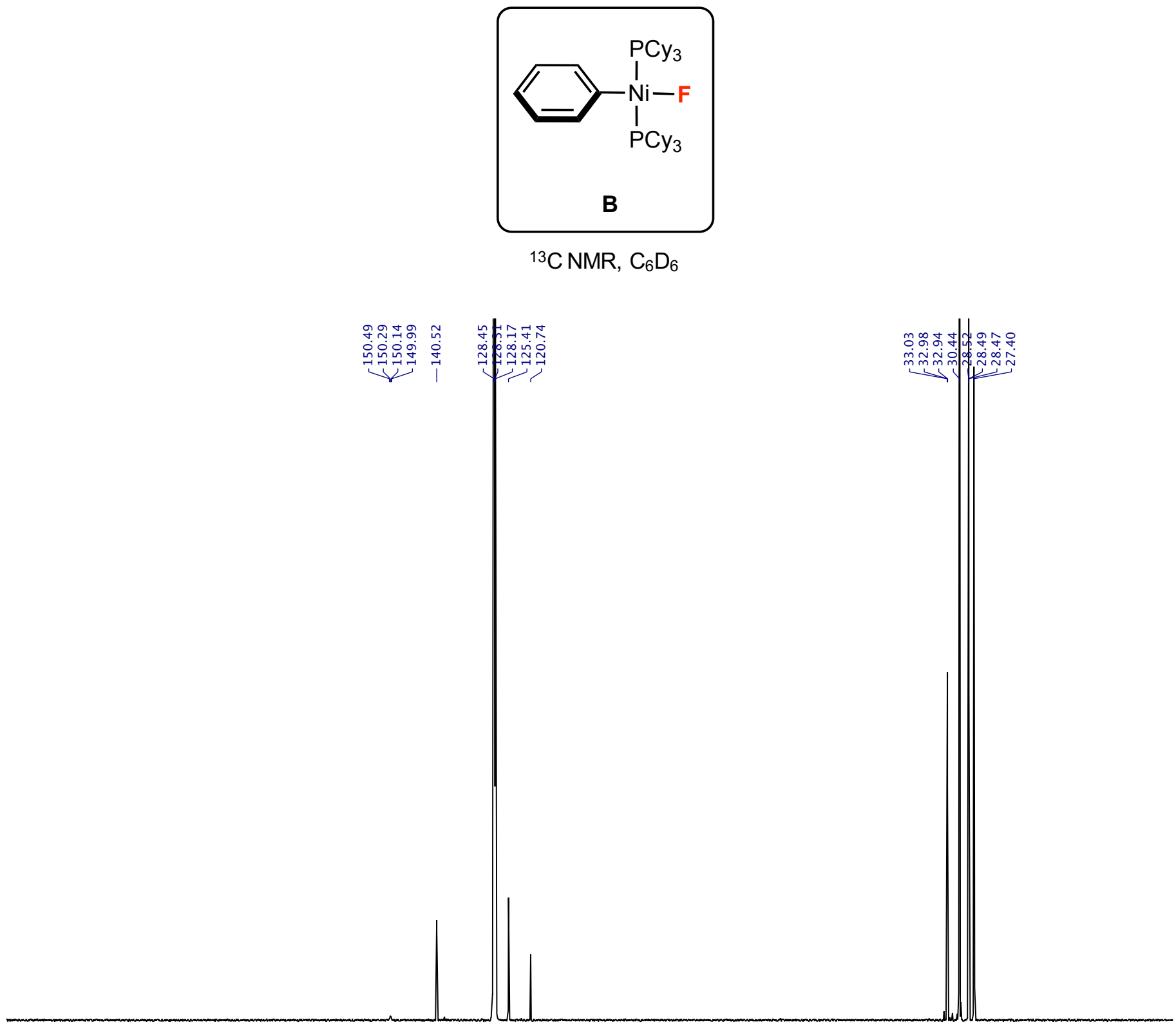

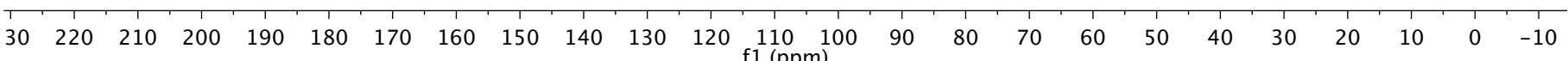




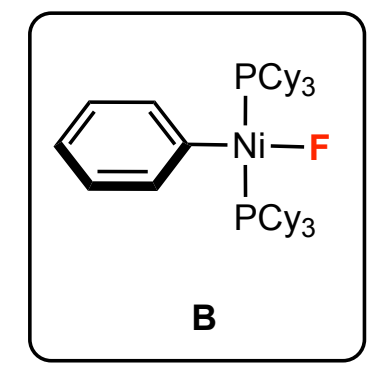

${ }^{31} \mathrm{P} N M R, \mathrm{C}_{6} \mathrm{D}_{6}$

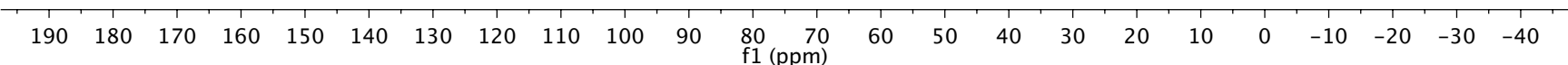




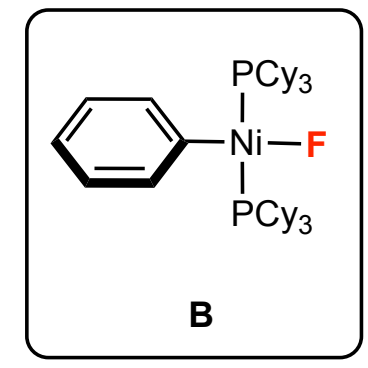

${ }^{19} \mathrm{~F}$ NMR (stored undernitrogen)

${ }^{19} \mathrm{~F} \mathrm{NMR}, \mathrm{C}_{6} \mathrm{D}_{6}$

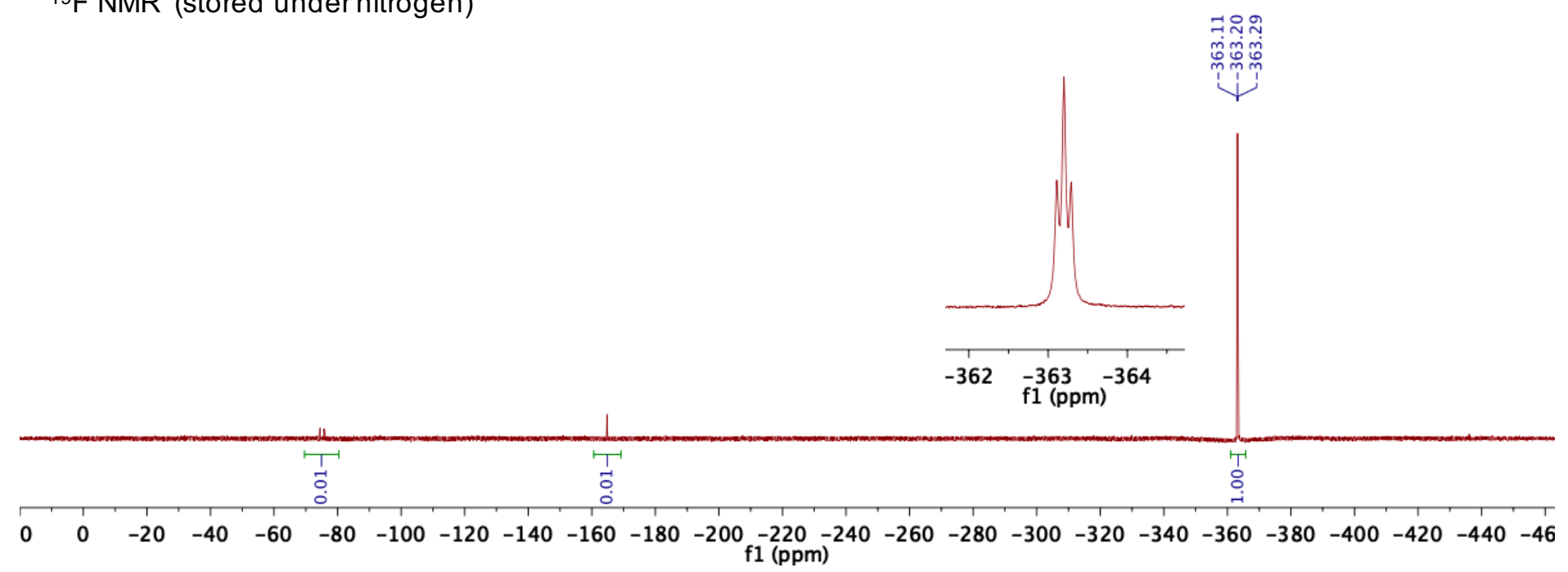

${ }^{19} \mathrm{~F}$ NMR (stored under air for 7 days)

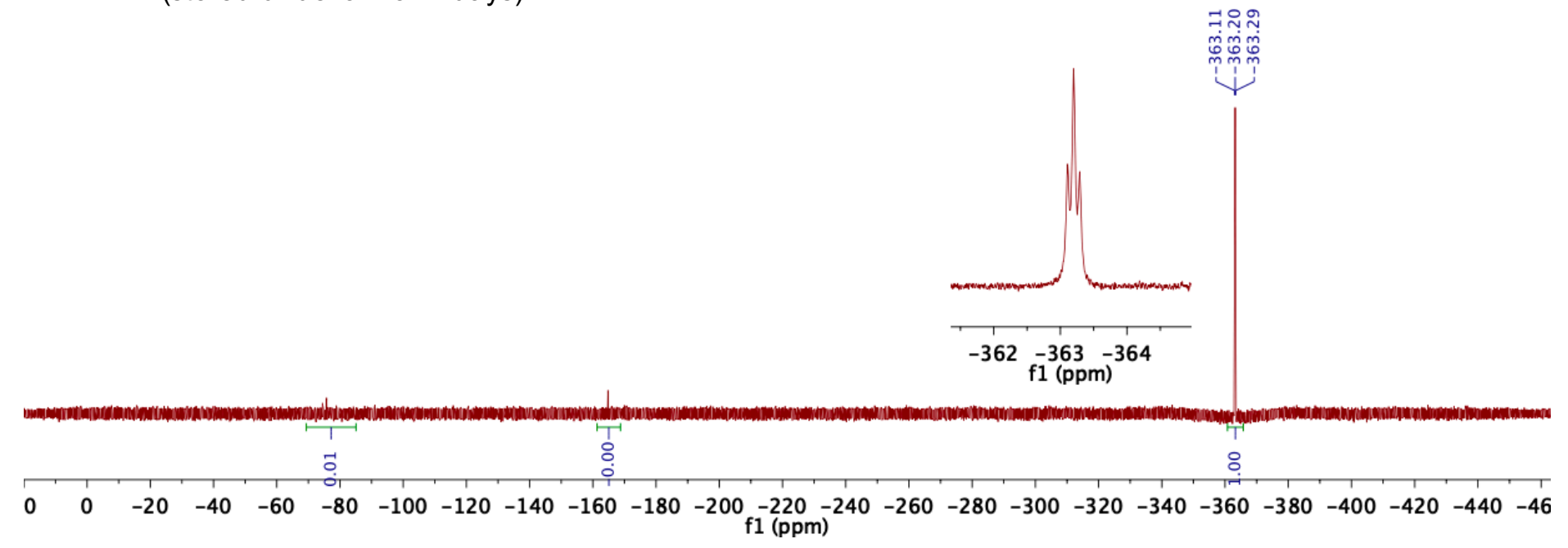




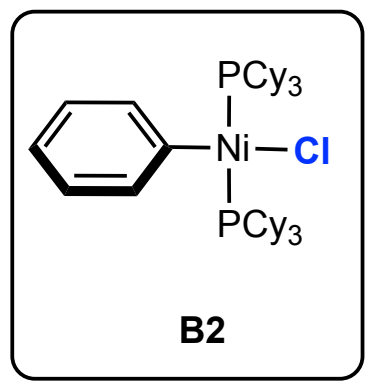

${ }^{1} \mathrm{H} N M R, \mathrm{C}_{6} \mathrm{D}_{6}$

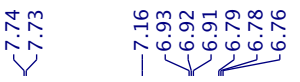

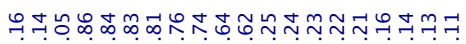

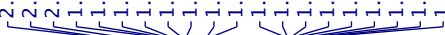

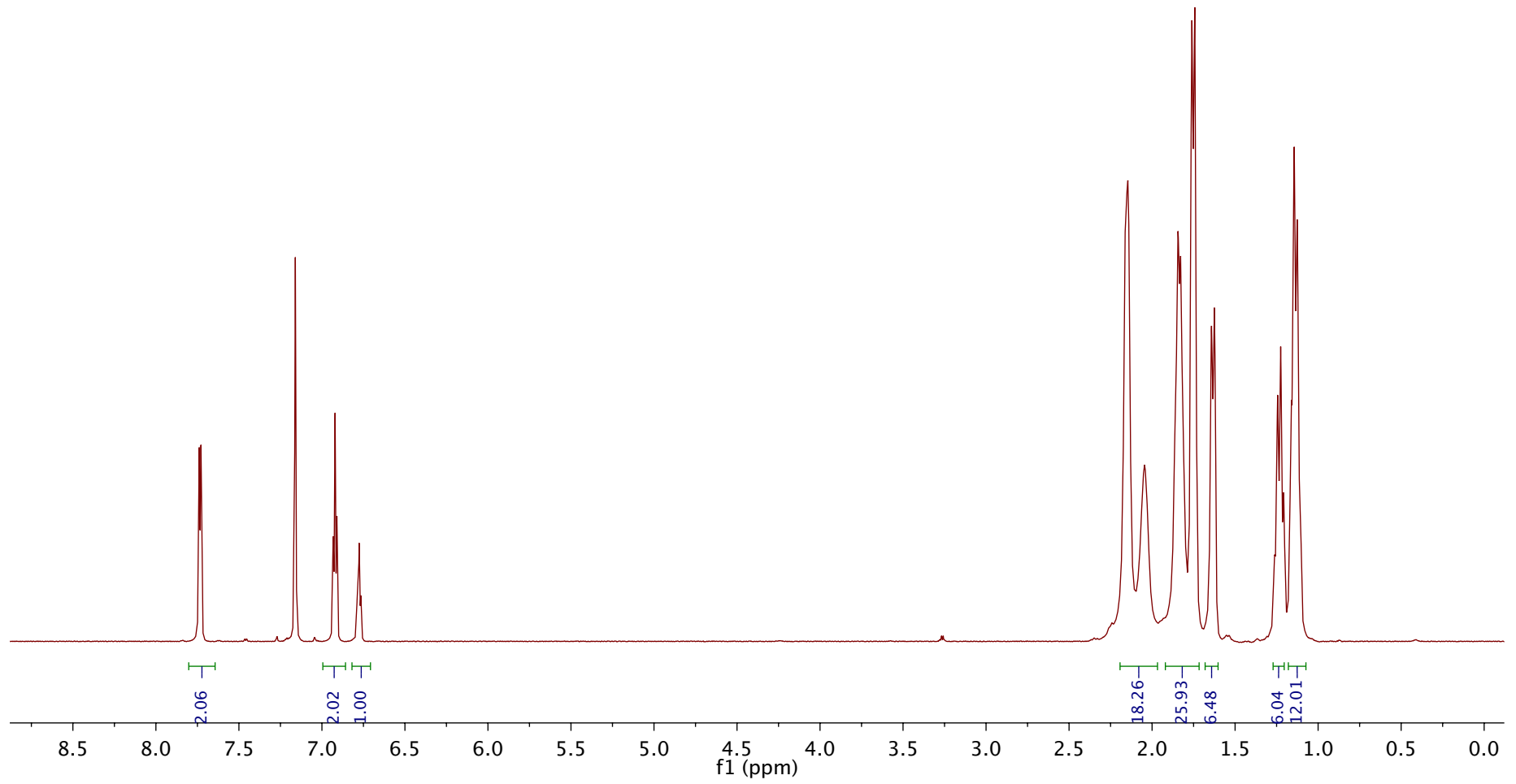



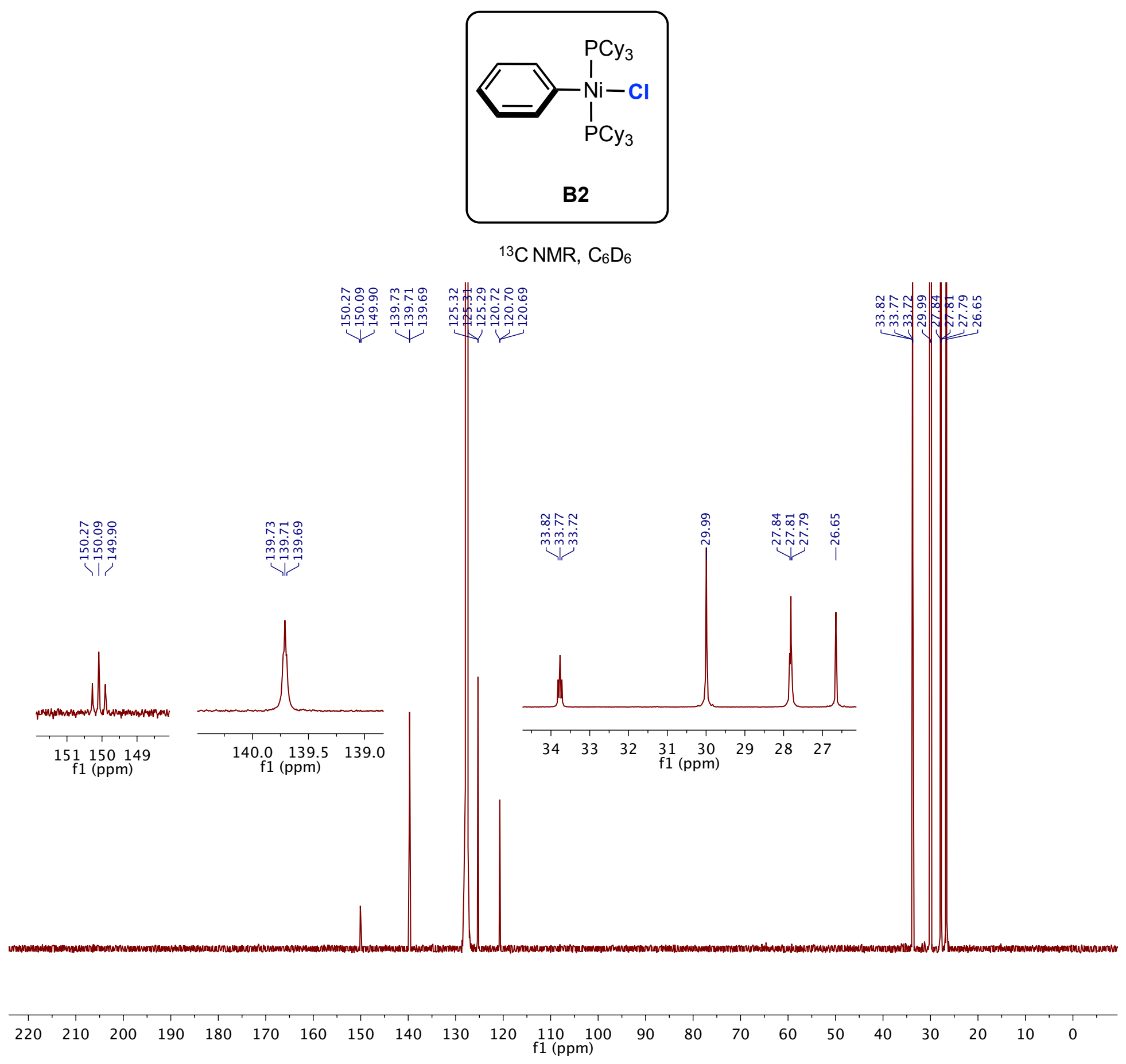


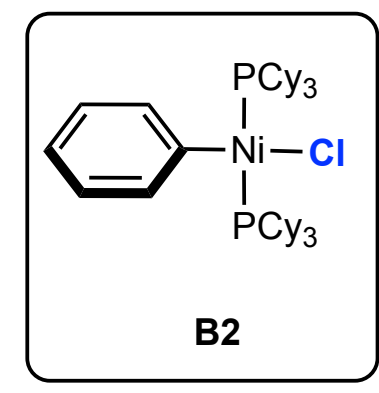

${ }^{31} \mathrm{P} N M R, \mathrm{C}_{6} \mathrm{D}_{6}$

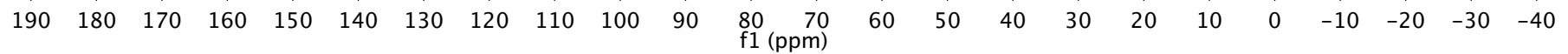




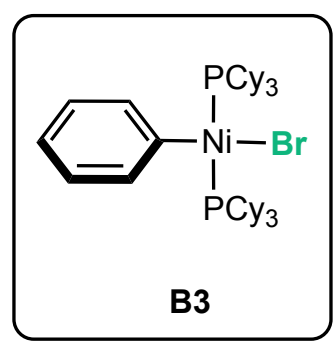

${ }^{1} \mathrm{H} N M R, \mathrm{C}_{6} \mathrm{D}_{6}$

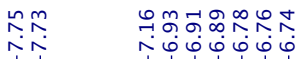

î́

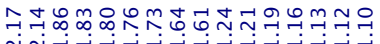

N

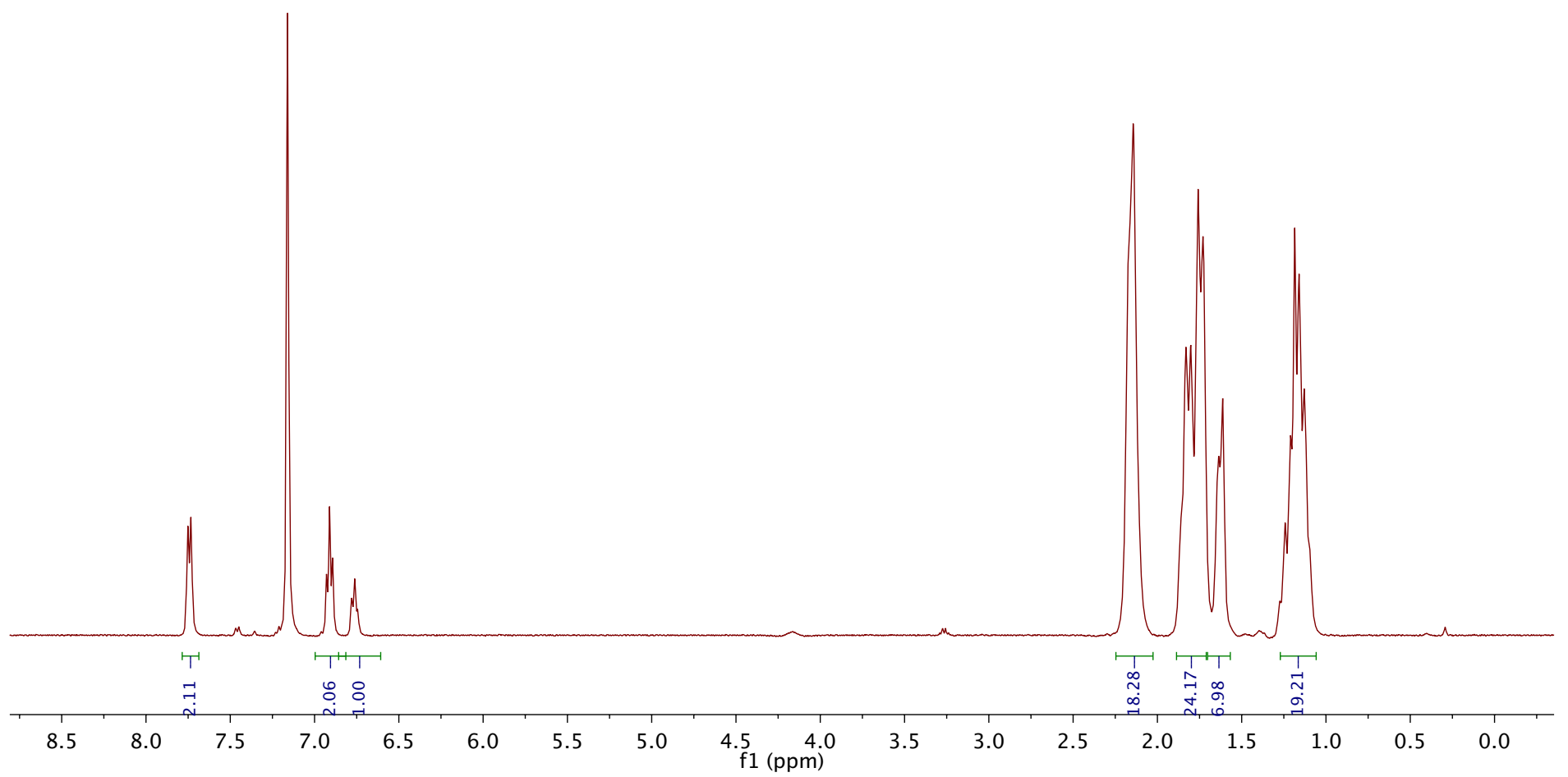




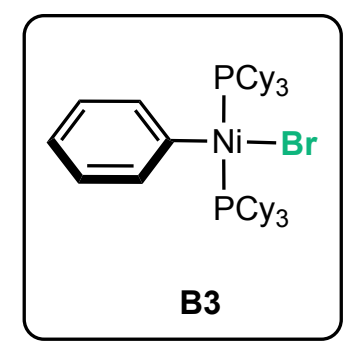

${ }^{13} \mathrm{C}$ NMR, $\mathrm{C}_{6} \mathrm{D}_{6}$

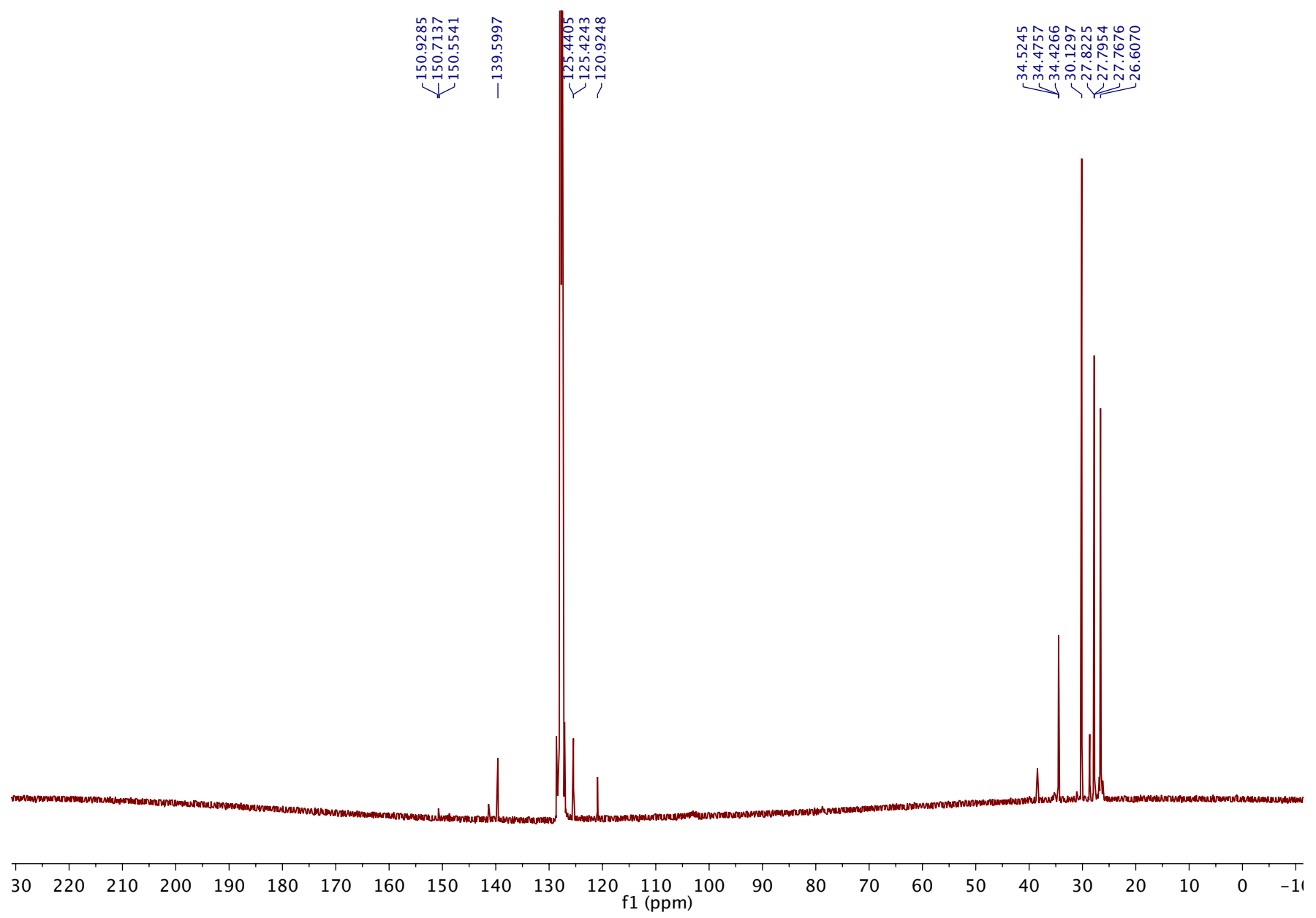




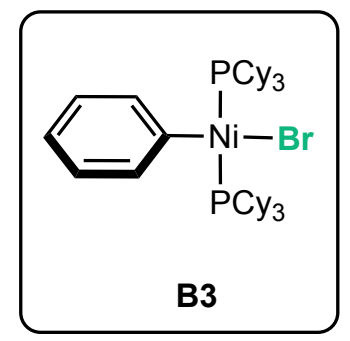

${ }^{31} \mathrm{P}$ NMR, $\mathrm{C}_{6} \mathrm{D}_{6}$

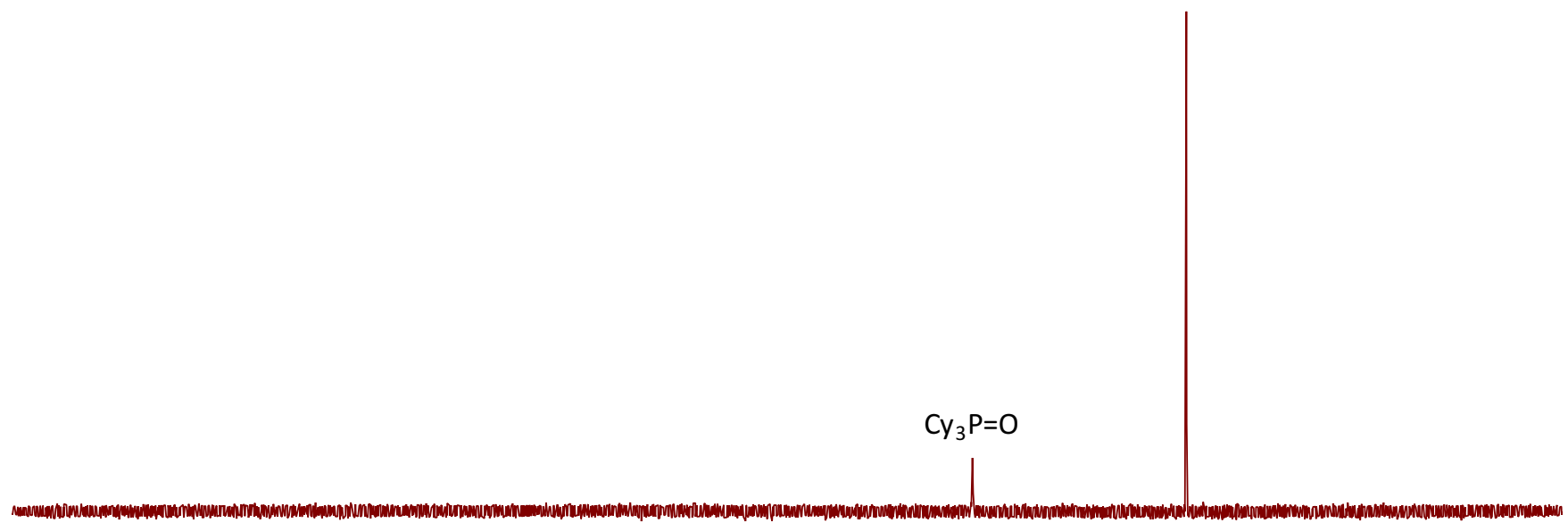

$\begin{array}{llllllllllllllllllllllll}190 & 180 & 170 & 160 & 150 & 140 & 130 & 120 & 110 & 100 & 90 & 80 & 70 & 60 & 50 & 40 & 30 & 20 & 10 & 0 & -10 & -20 & -30 & -40\end{array}$




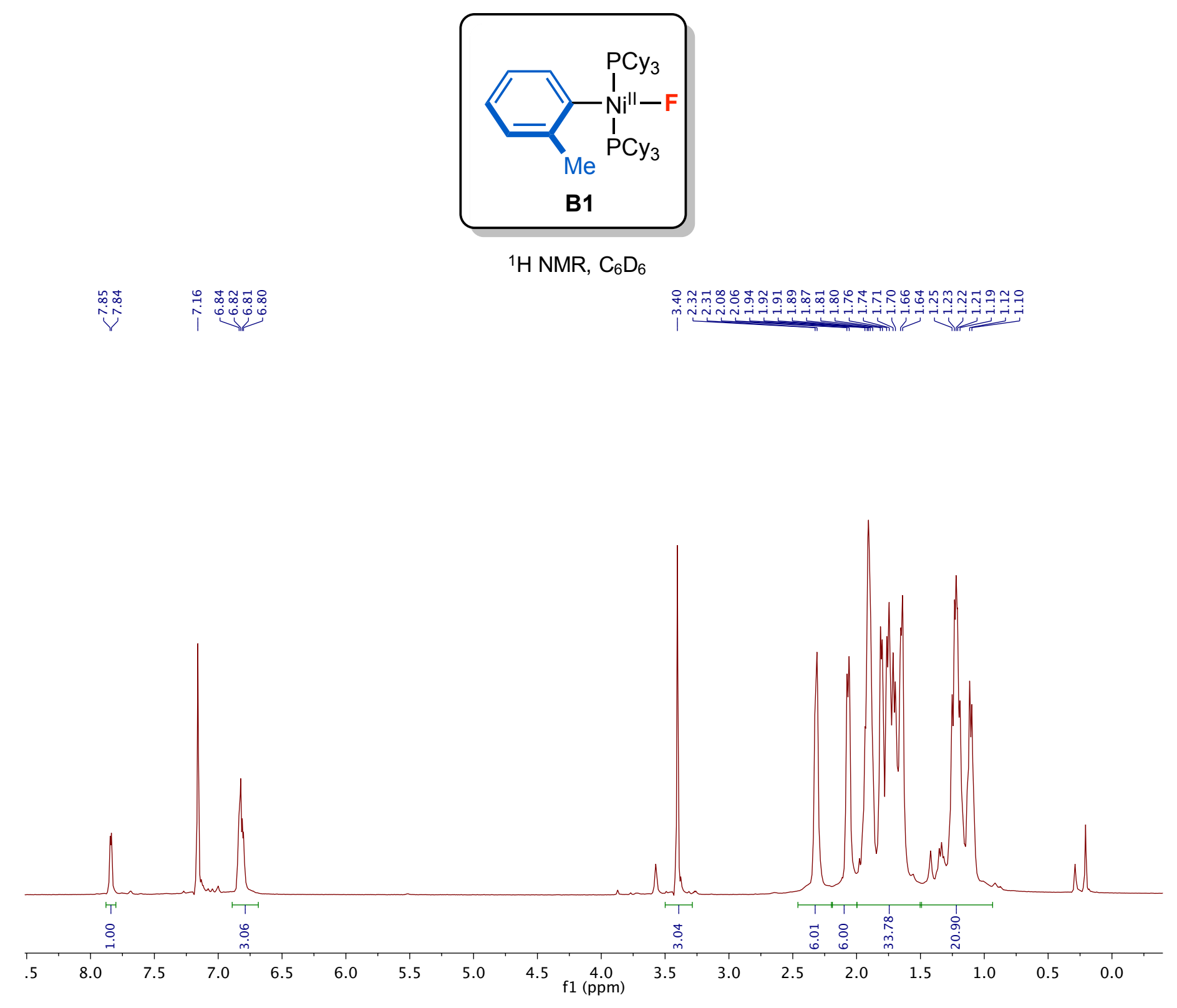




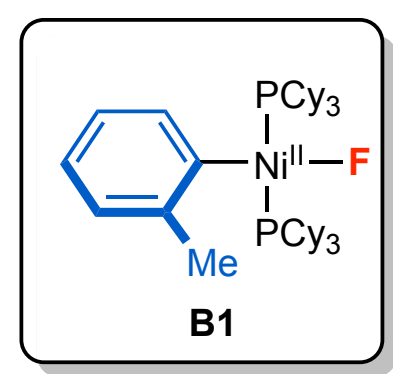

${ }^{13} \mathrm{C}$ NMR, $\mathrm{C}_{6} \mathrm{D}_{6}$

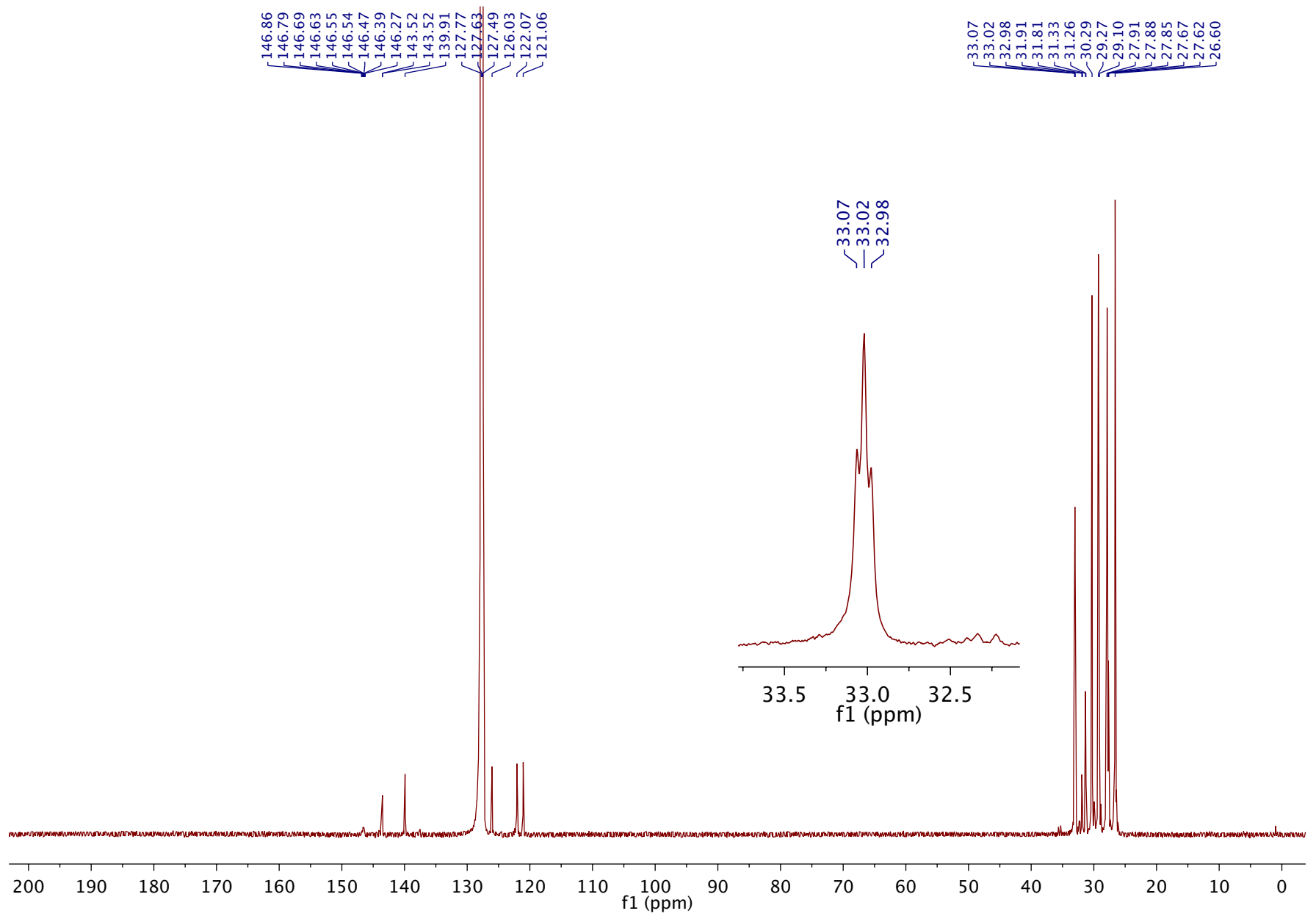




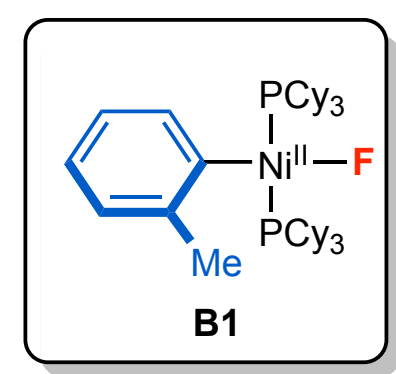

${ }^{31} \mathrm{P} N M R, \mathrm{C}_{6} \mathrm{D}_{6}$
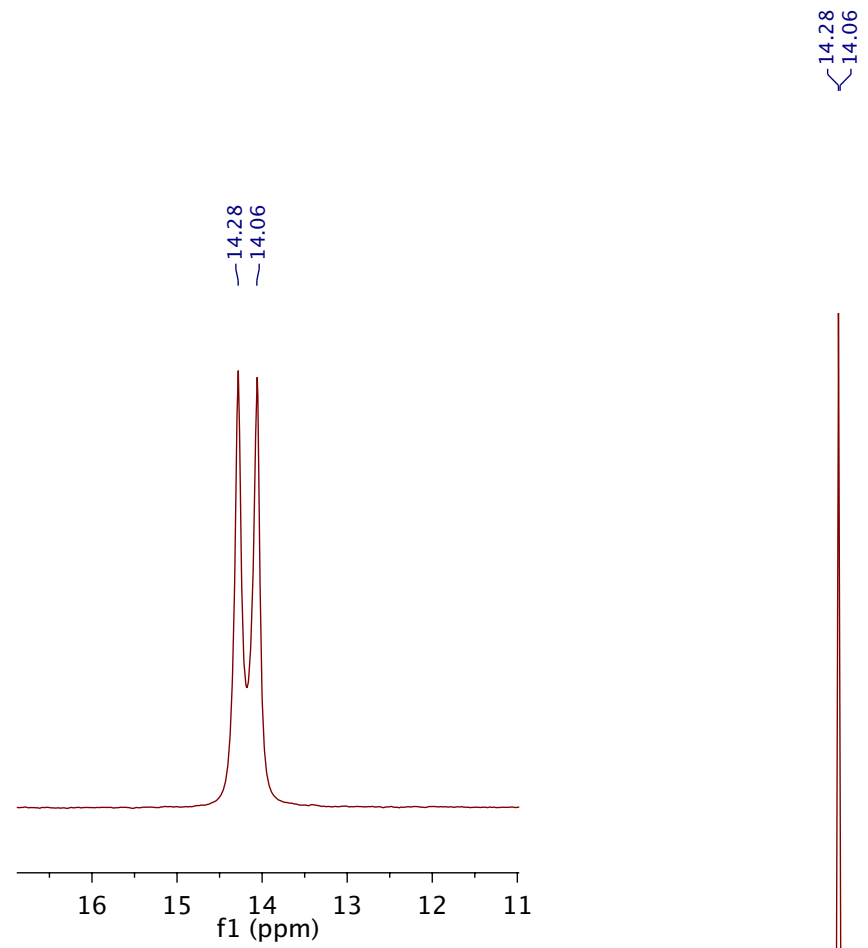

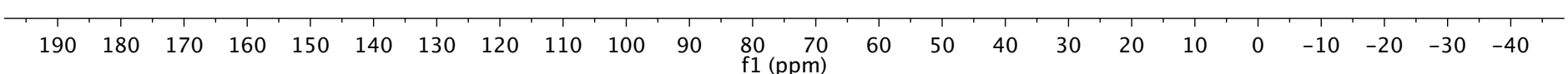




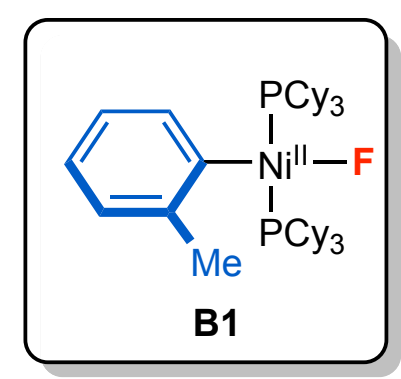

${ }^{19} \mathrm{~F} N M R, \mathrm{C}_{6} \mathrm{D}_{6}$

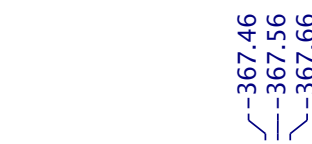

운ํㅠㅇ

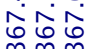

운유요

에용
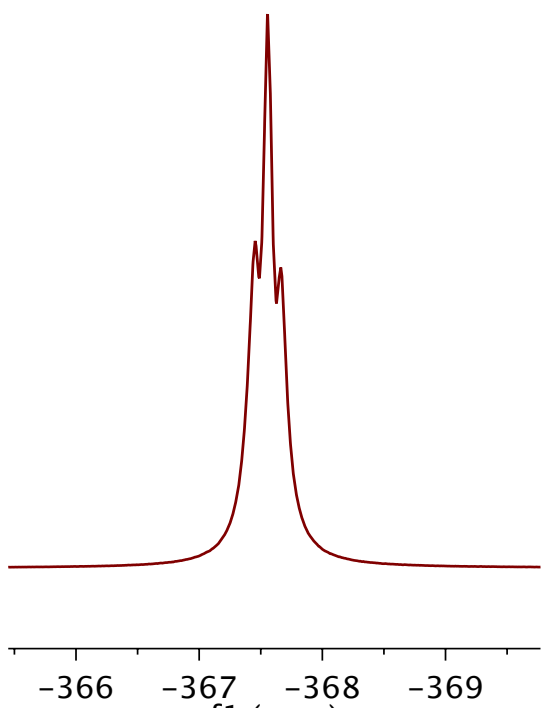

f1 (ppm)

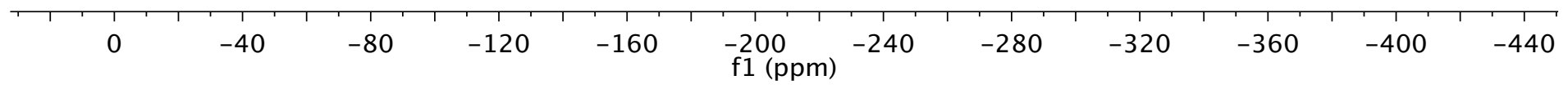




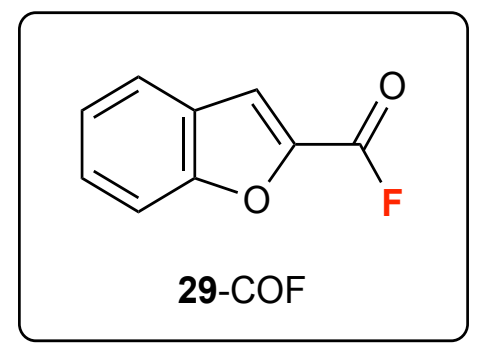

${ }^{1} \mathrm{H}$ NMR

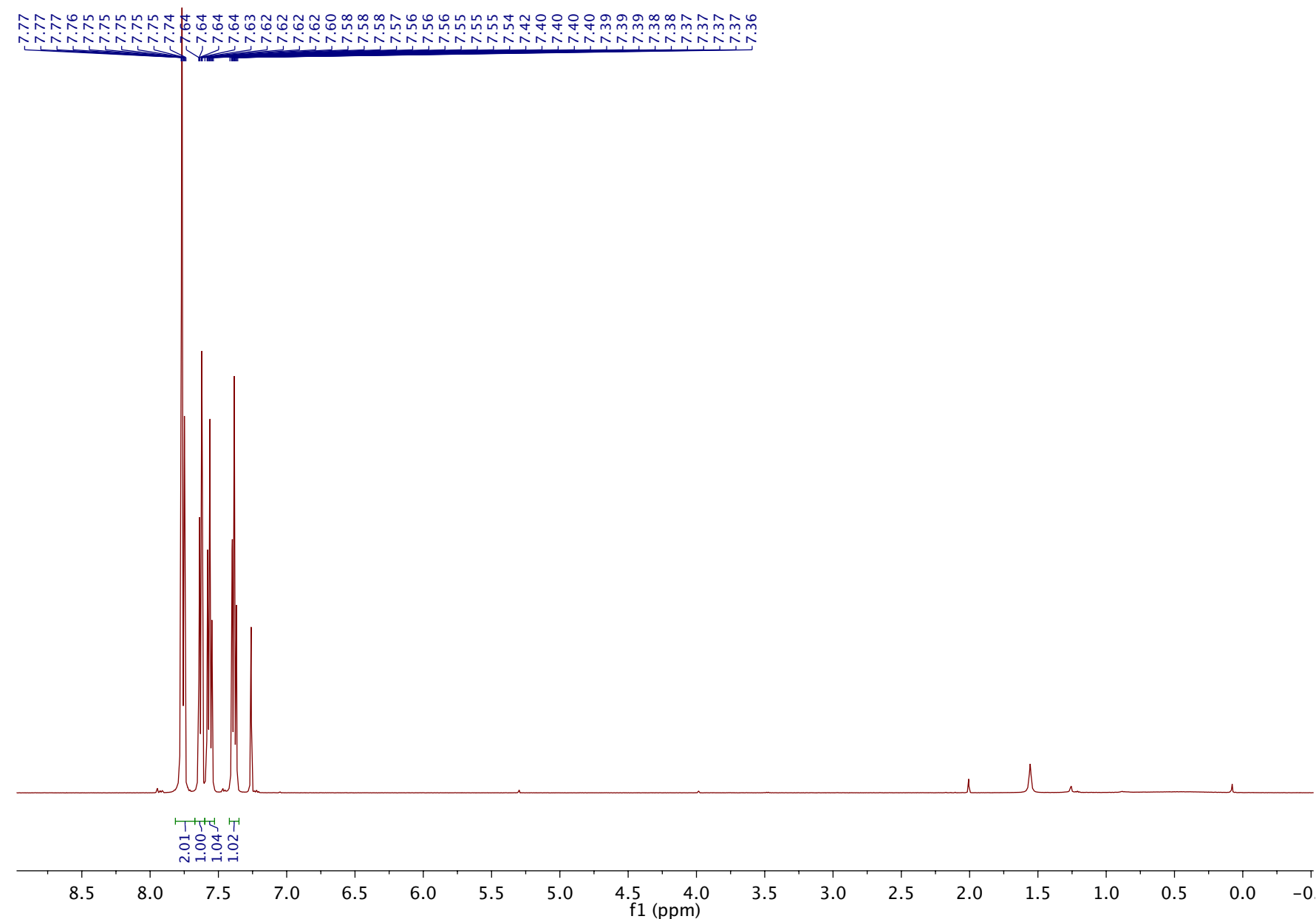




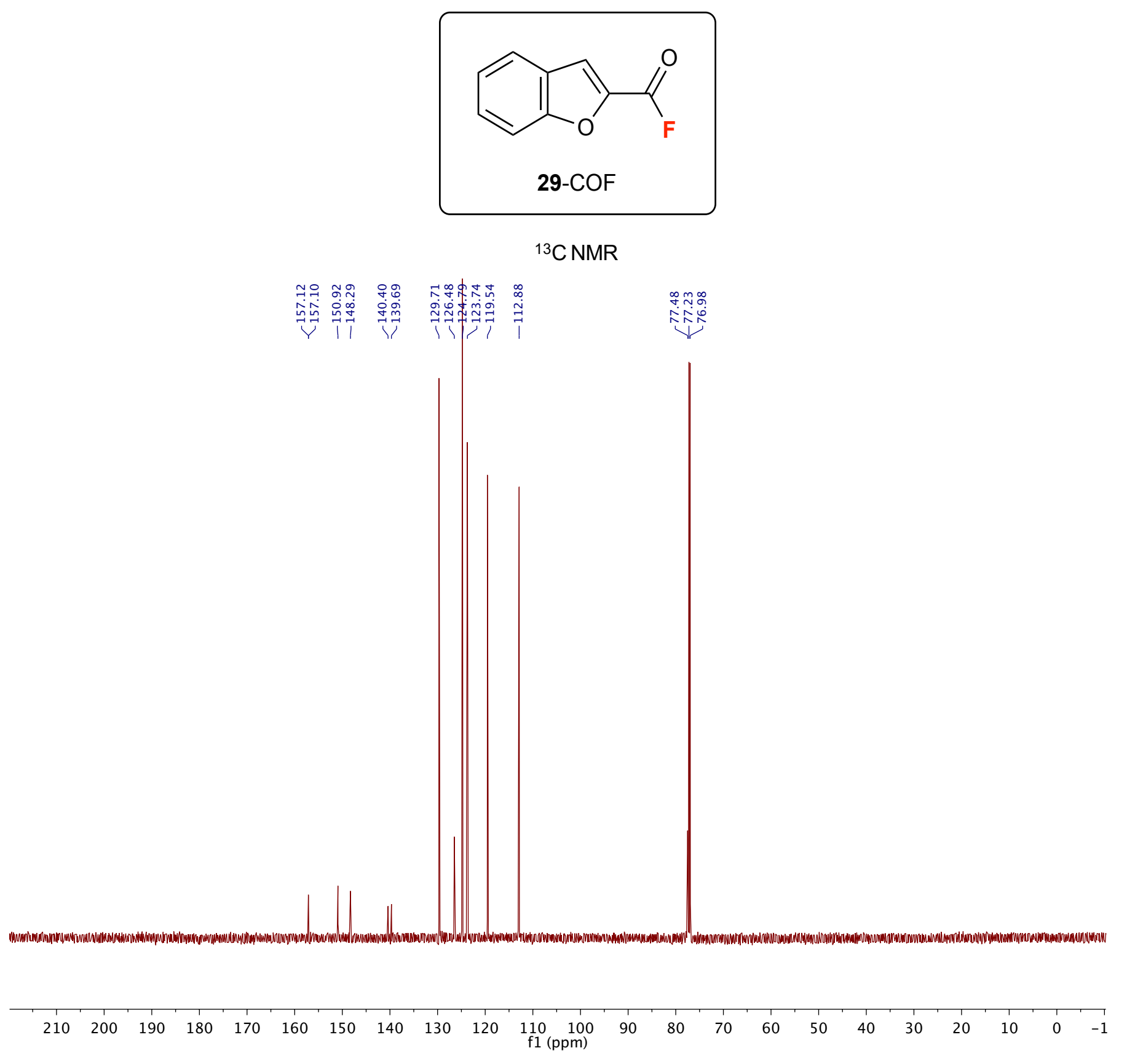




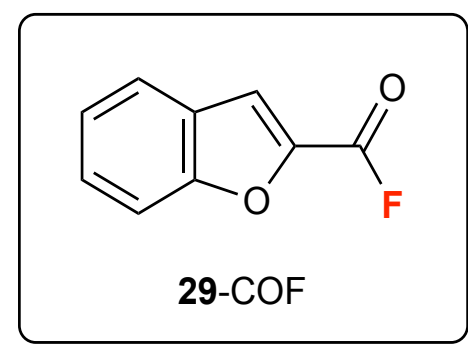

${ }^{19}$ F NMR

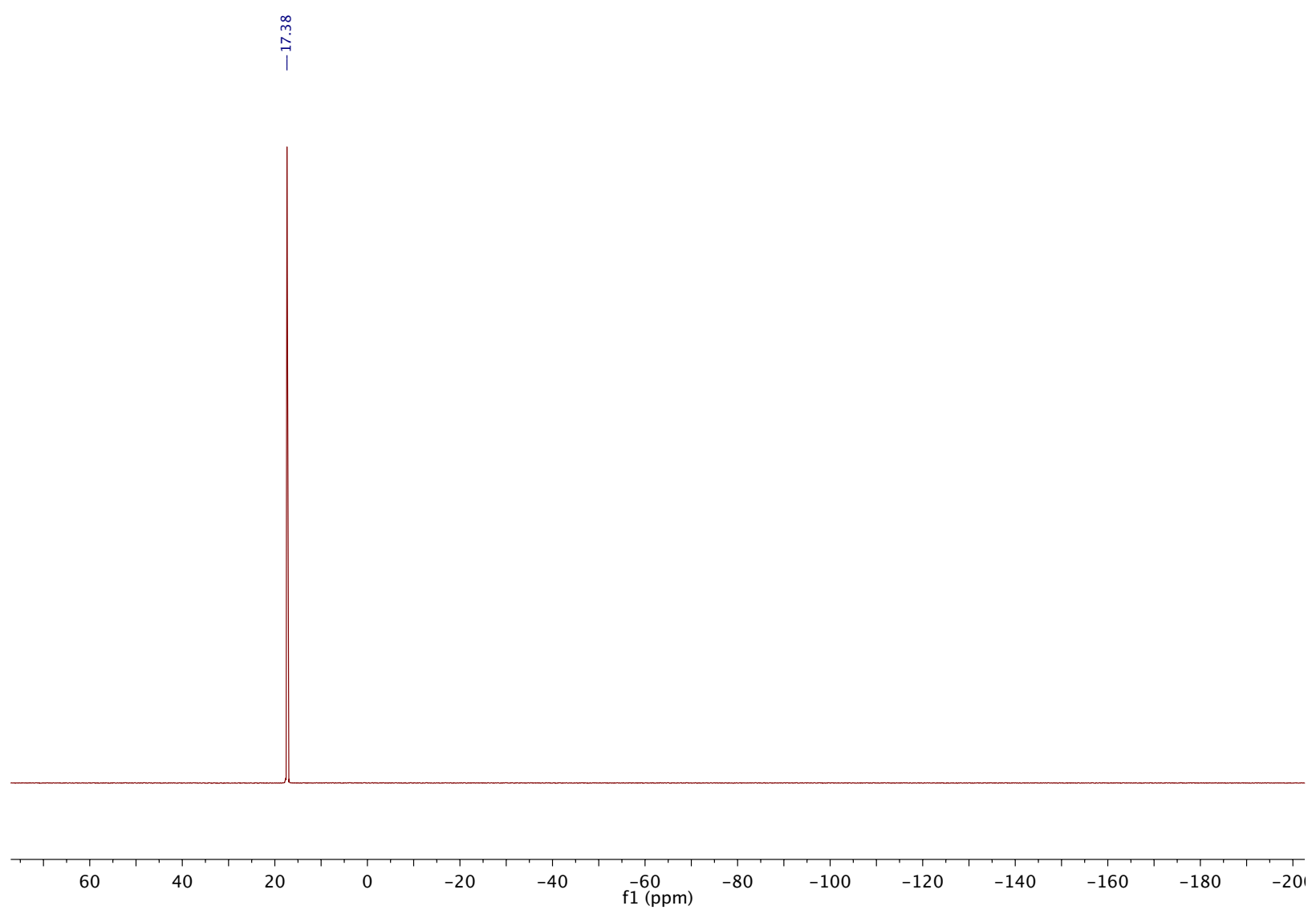




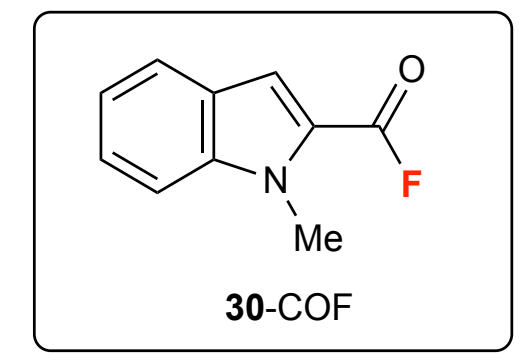

${ }^{1} \mathrm{H}$ NMR

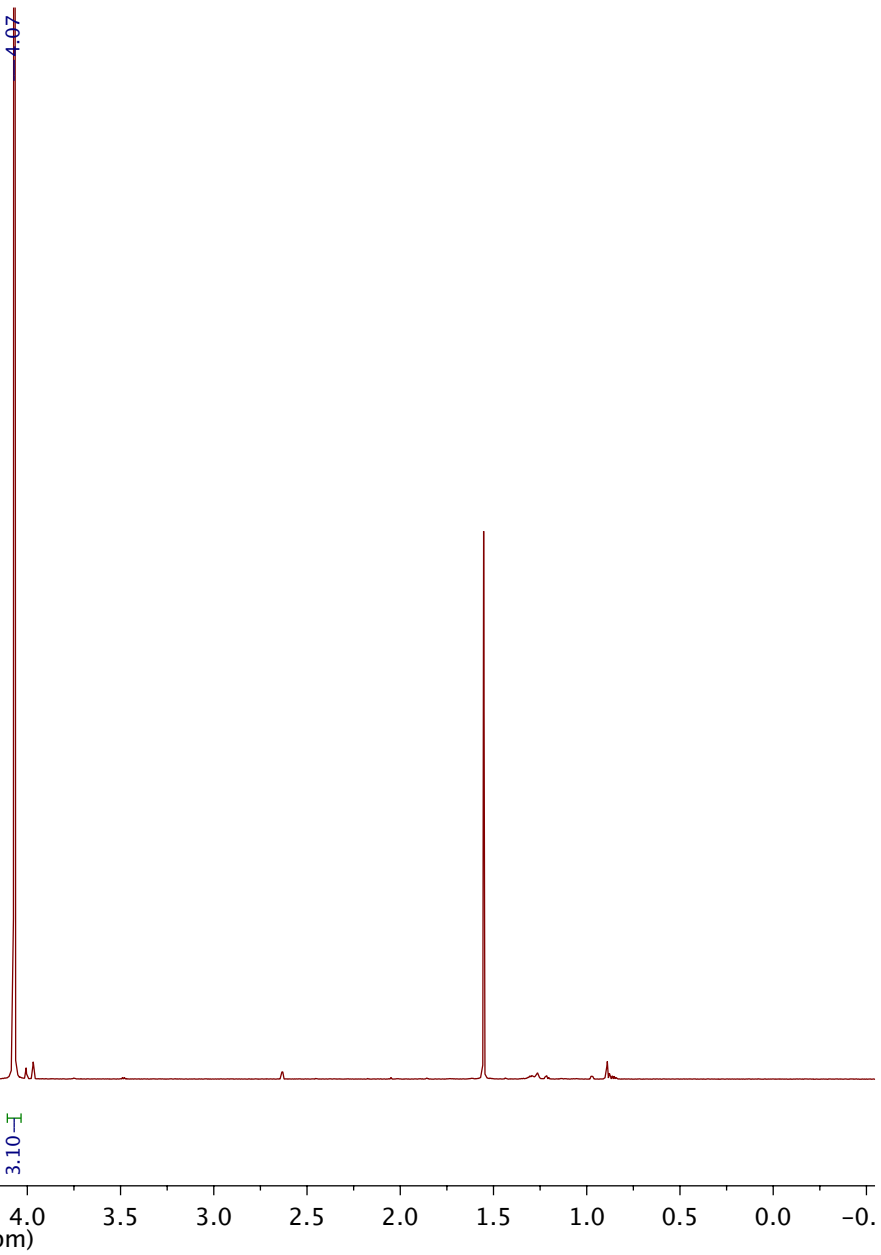




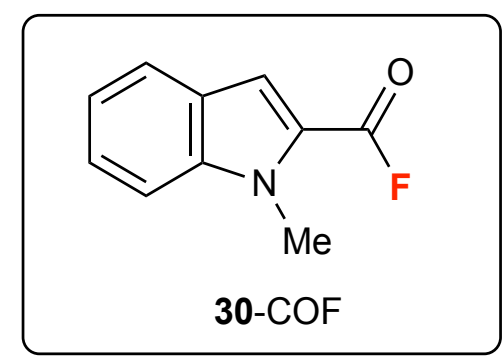

${ }^{13} \mathrm{C}$ NMR
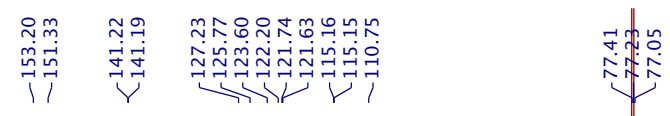

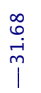

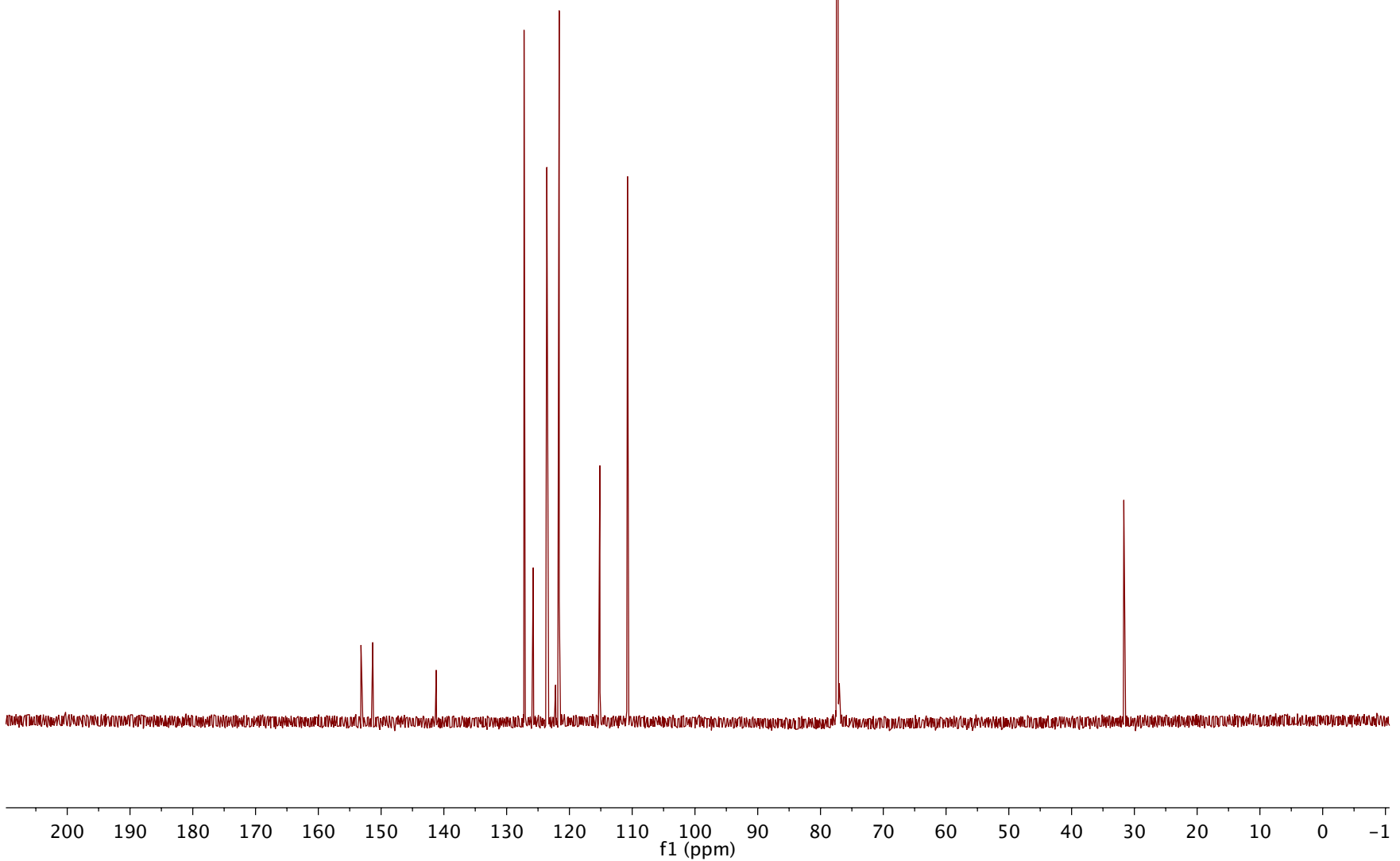




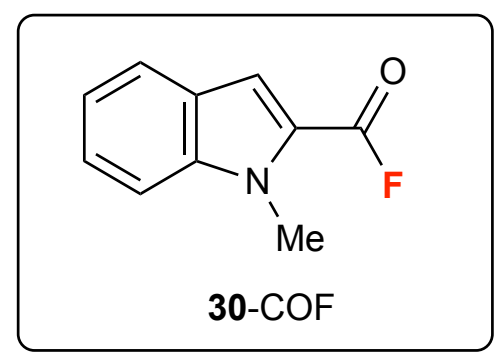

${ }^{19} \mathrm{~F} \mathrm{NMR}$

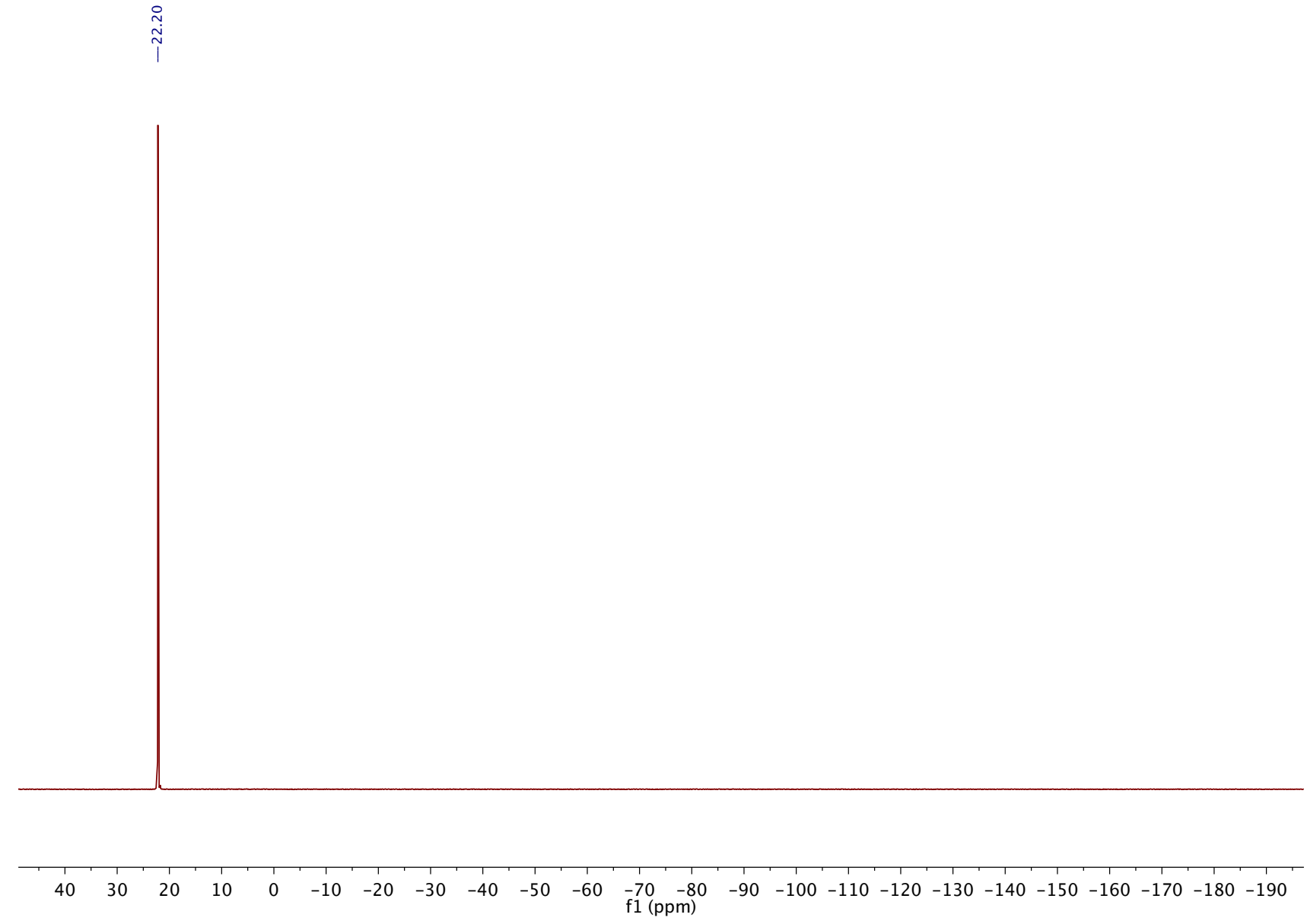




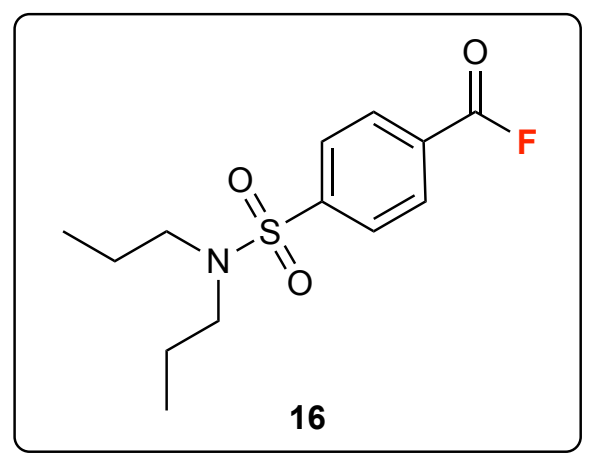

${ }^{1} \mathrm{H}$ NMR

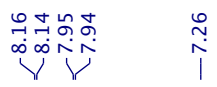

구우요용

ming

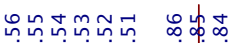

iinitiri ioj

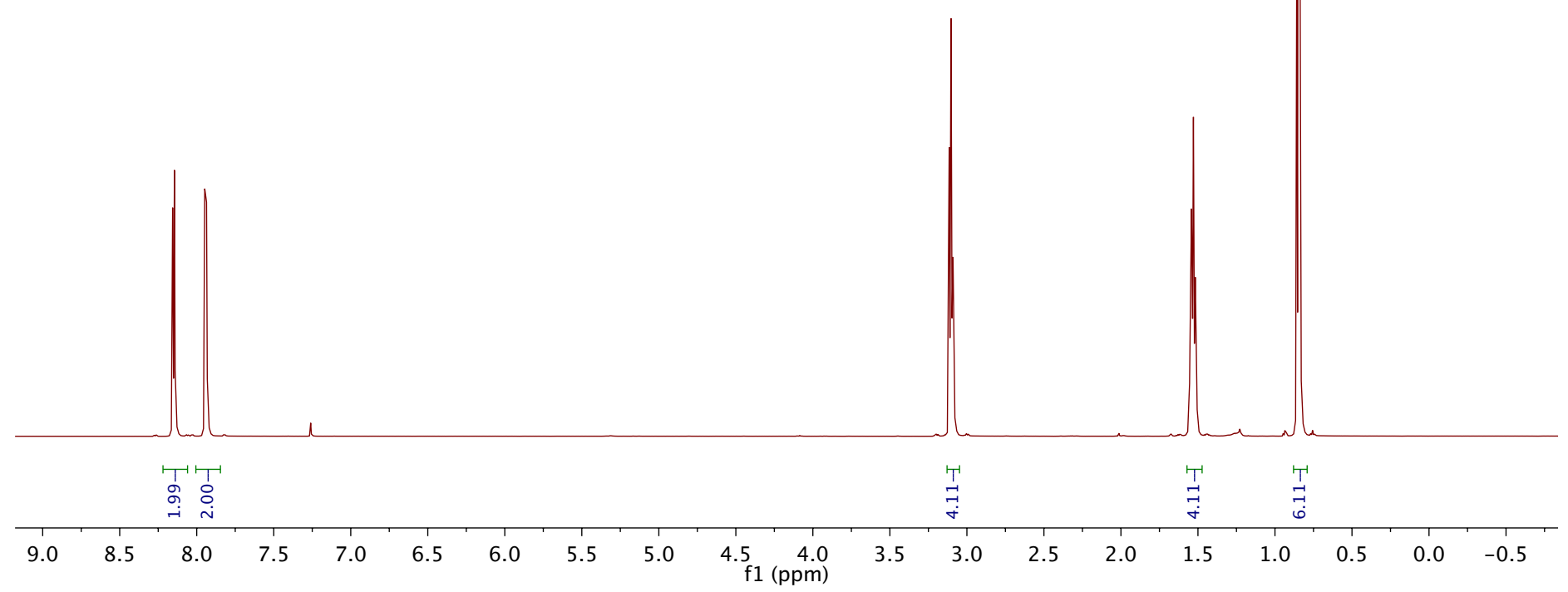



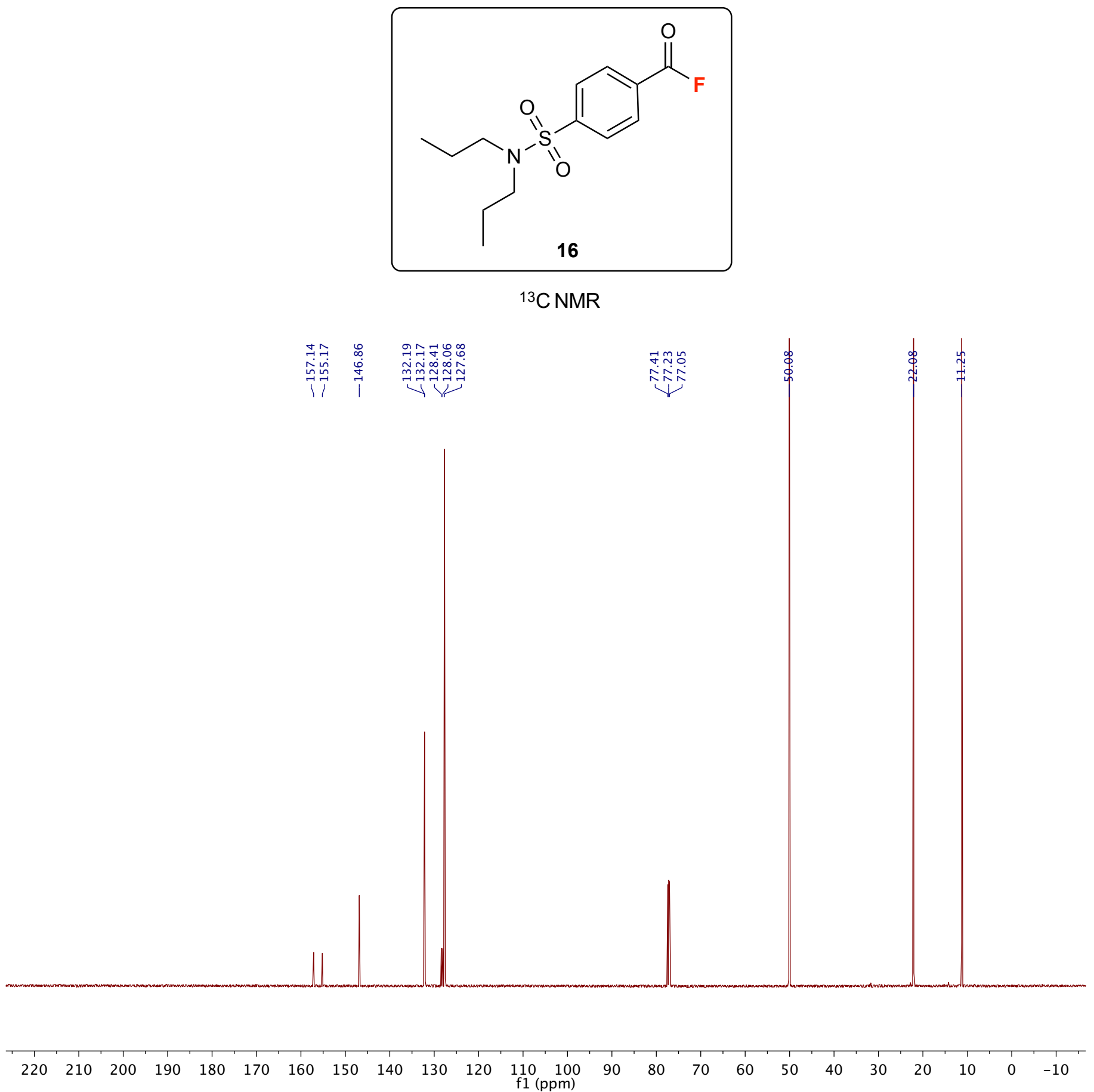


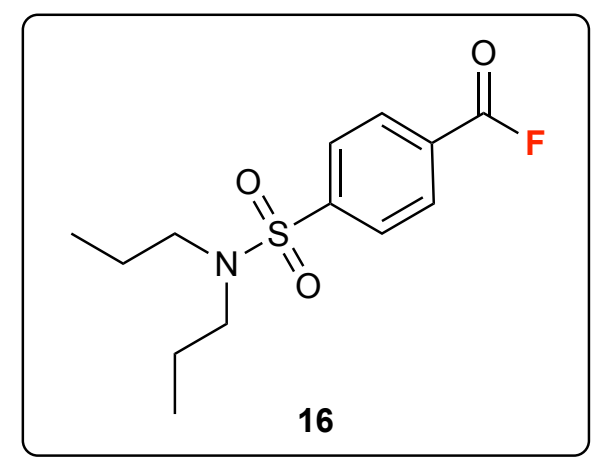

${ }^{19} \mathrm{~F} \mathrm{NMR}$

$\stackrel{\circ}{\stackrel{2}{*}}$

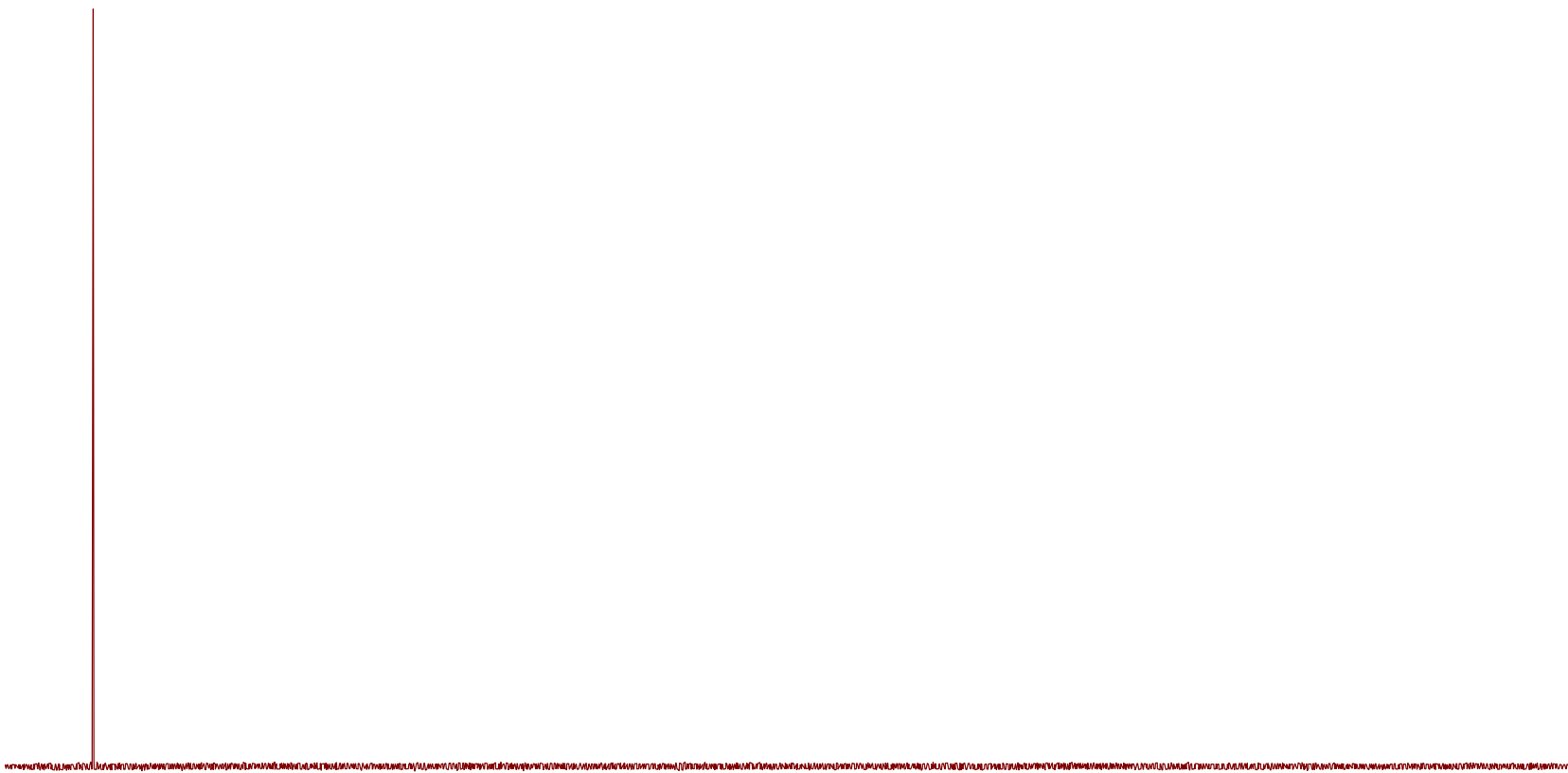

$\begin{array}{lllllllllllllllllllllllll}30 & 20 & 10 & 0 & -10 & -20 & -30 & -40 & -50 & -60 & -70 & -80 & -90 & -100 & -110 & -120 & -130 & -140 & -150 & -160 & -170 & -180 & -190 & -200\end{array}$ 


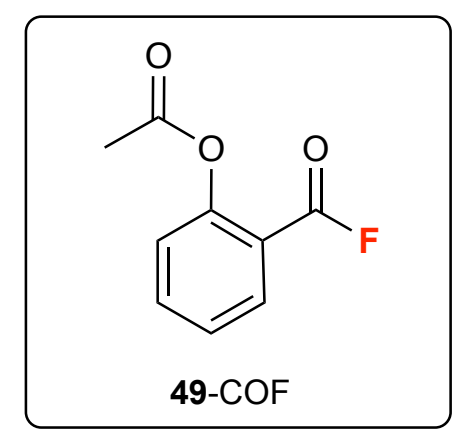

${ }^{1} \mathrm{H}$ NMR

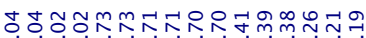

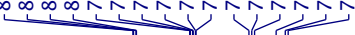

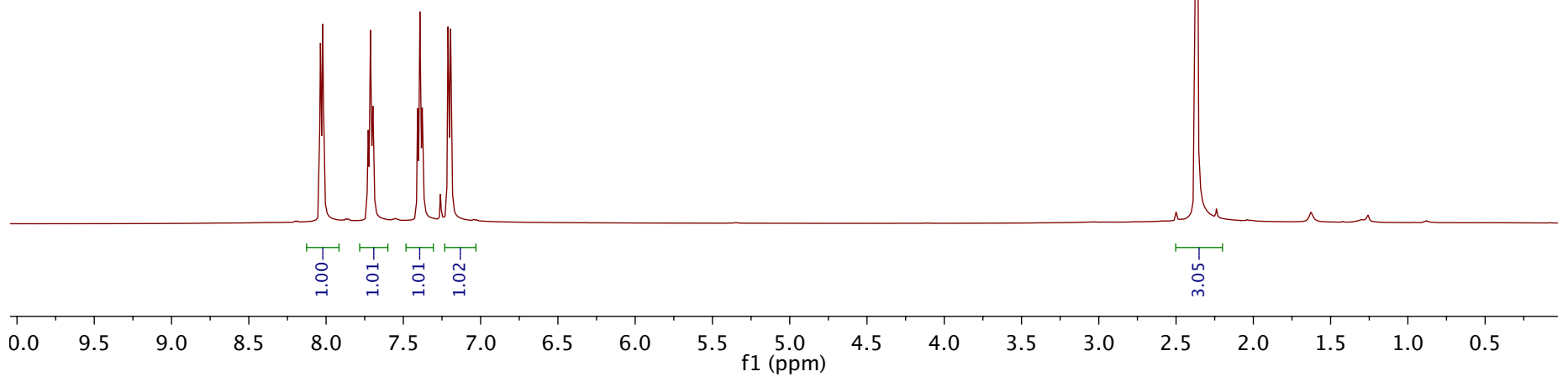




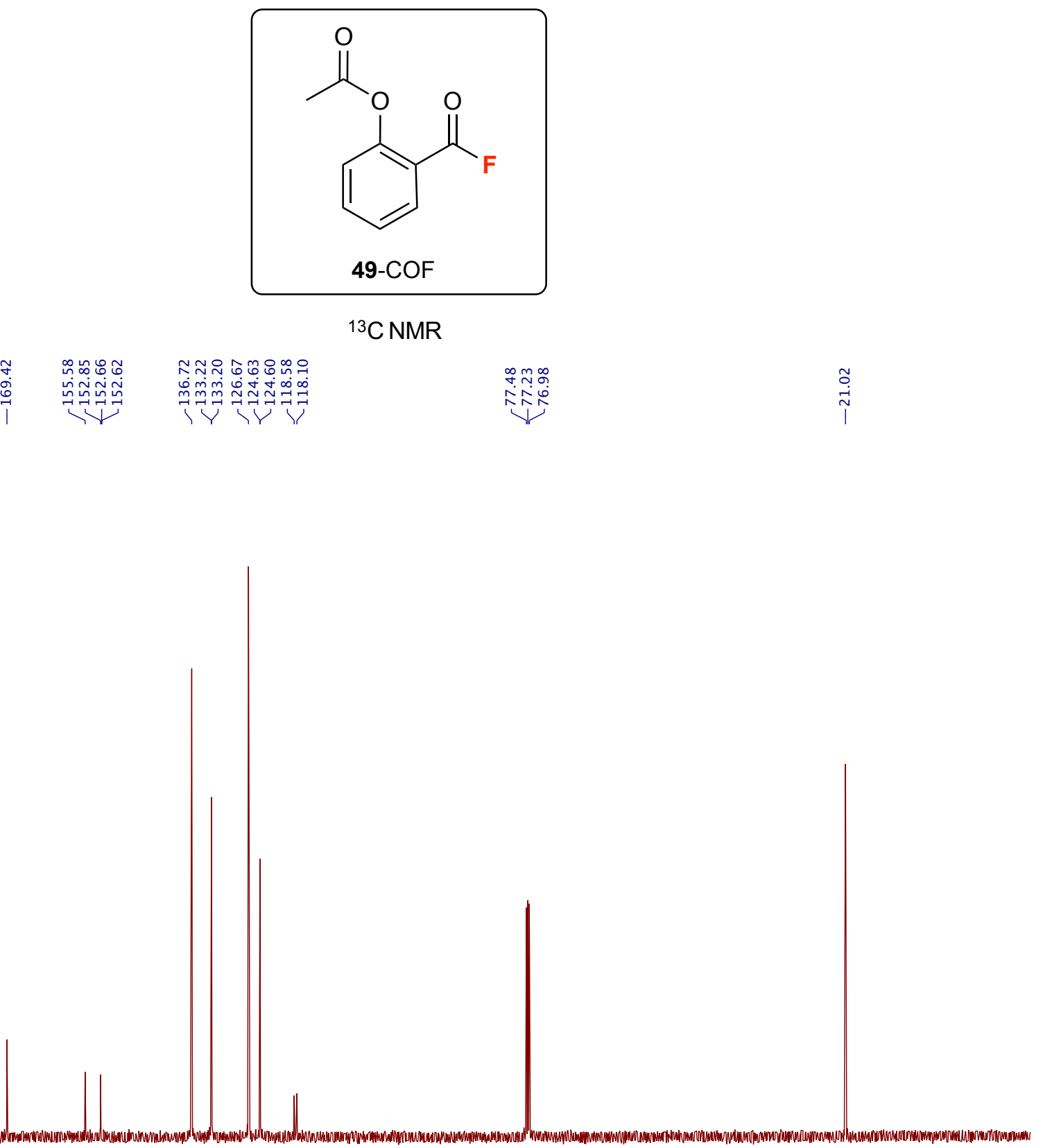

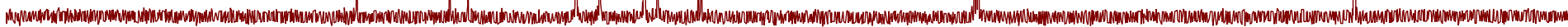

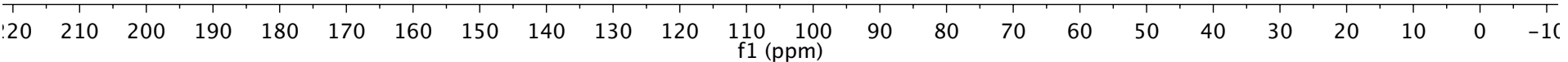




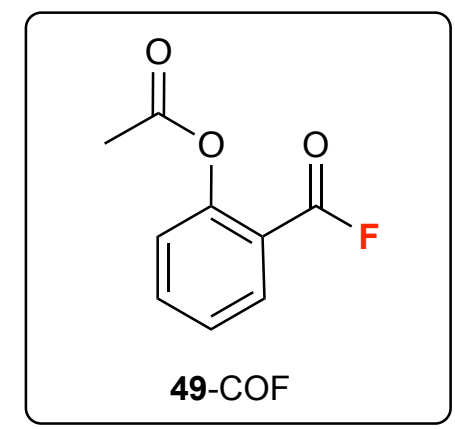

${ }^{19} \mathrm{~F} \mathrm{NMR}$

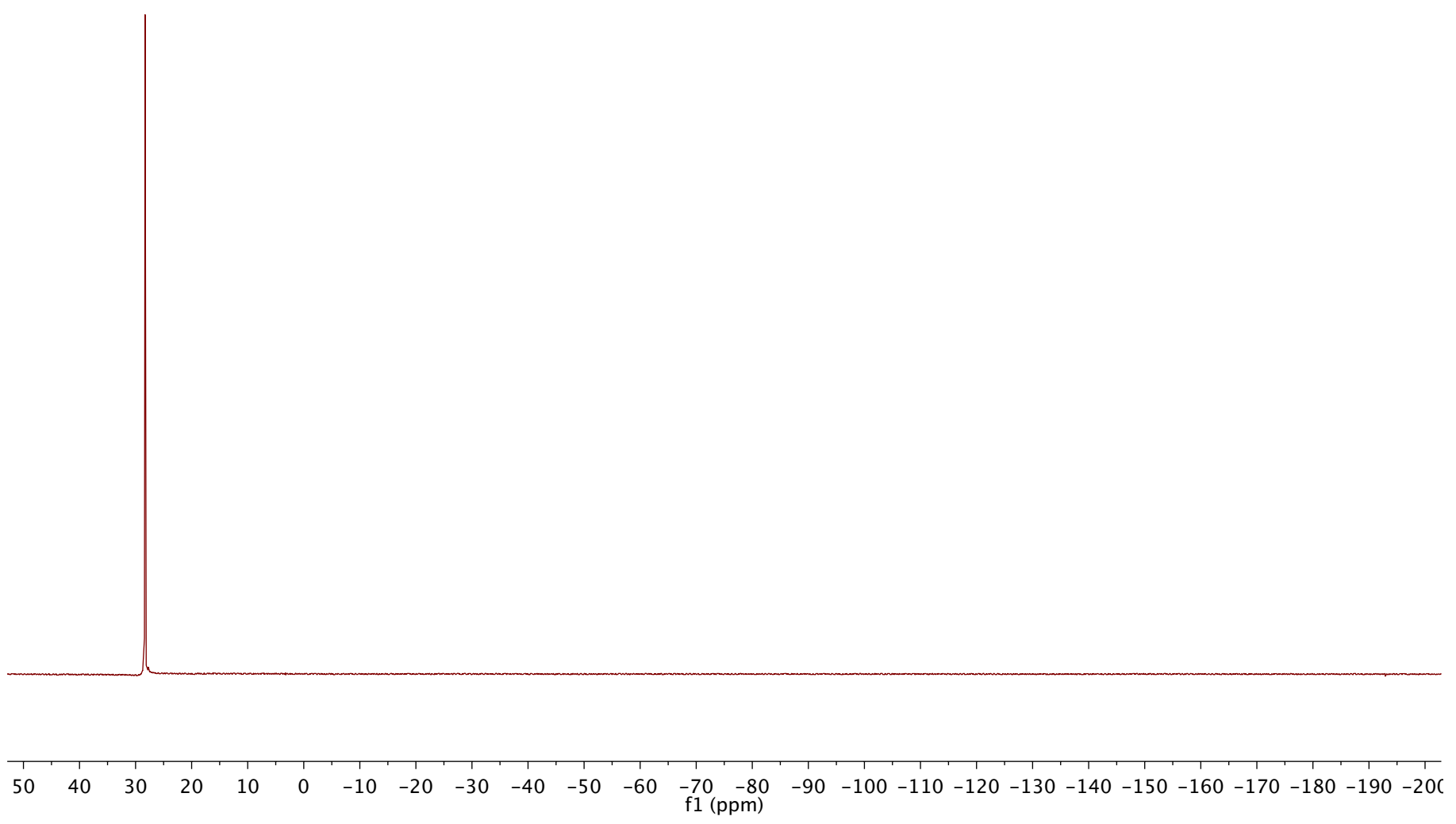




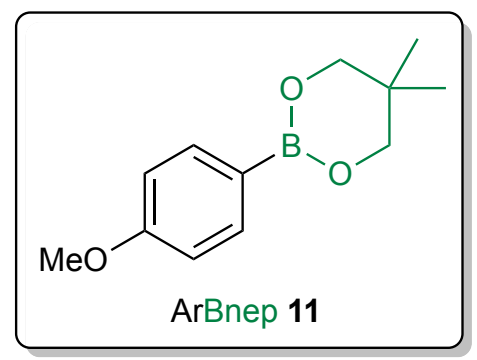

${ }^{1} \mathrm{H}$ NMR
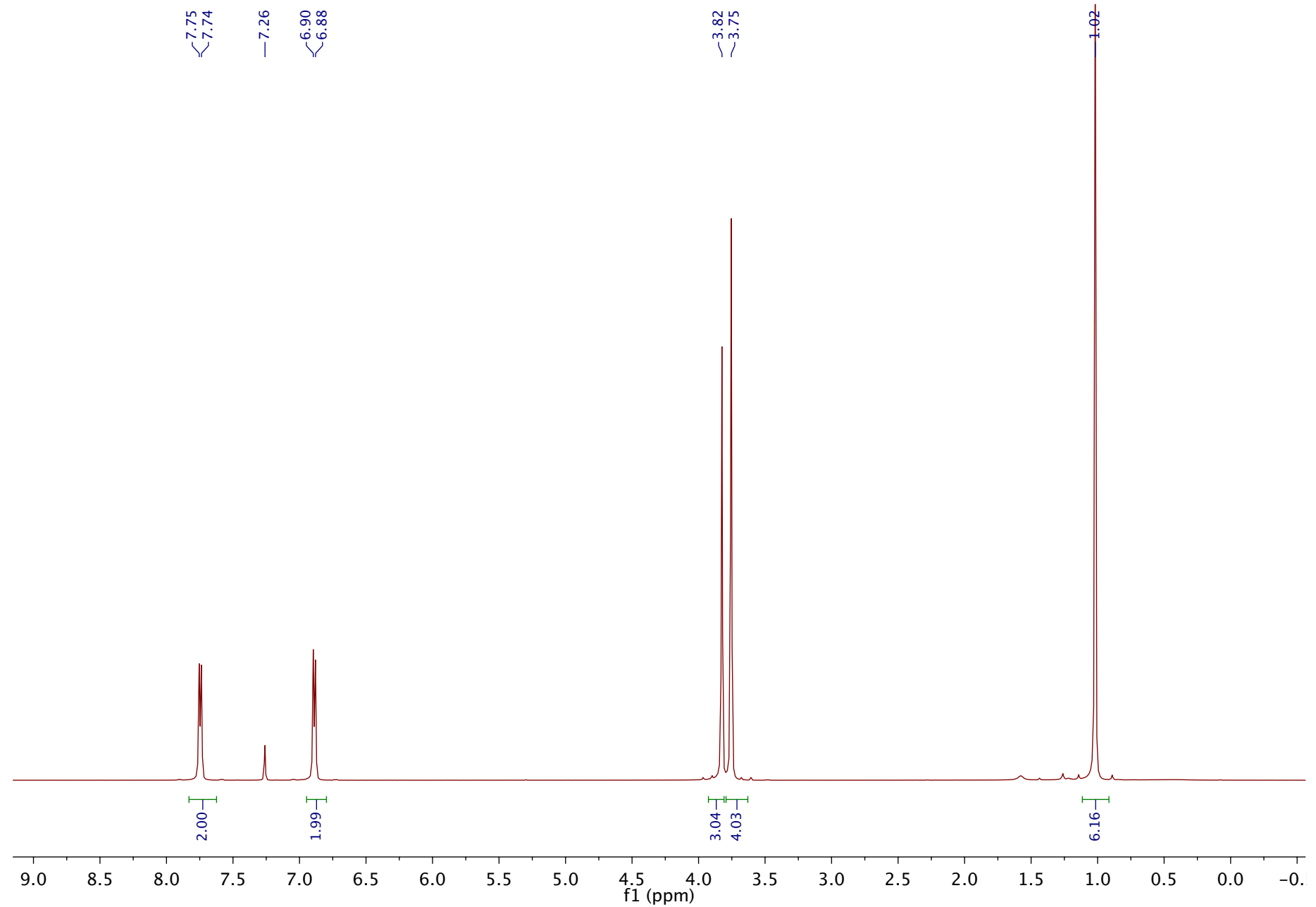


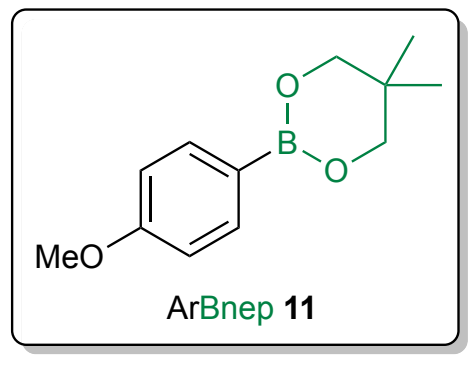

${ }^{13} \mathrm{C}$ NMR

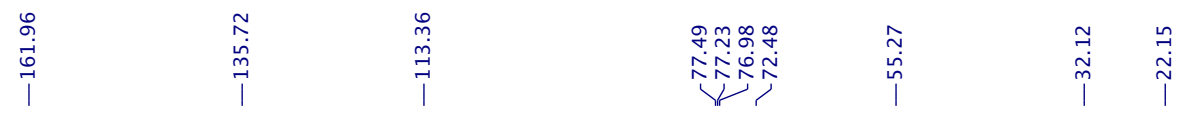

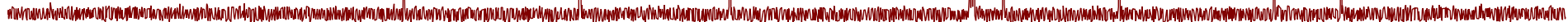

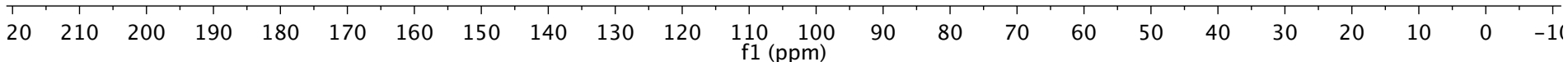




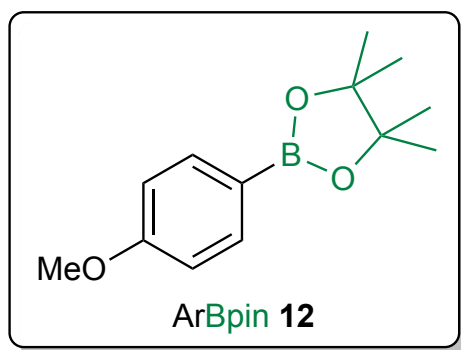

${ }^{1} \mathrm{H}$ NMR

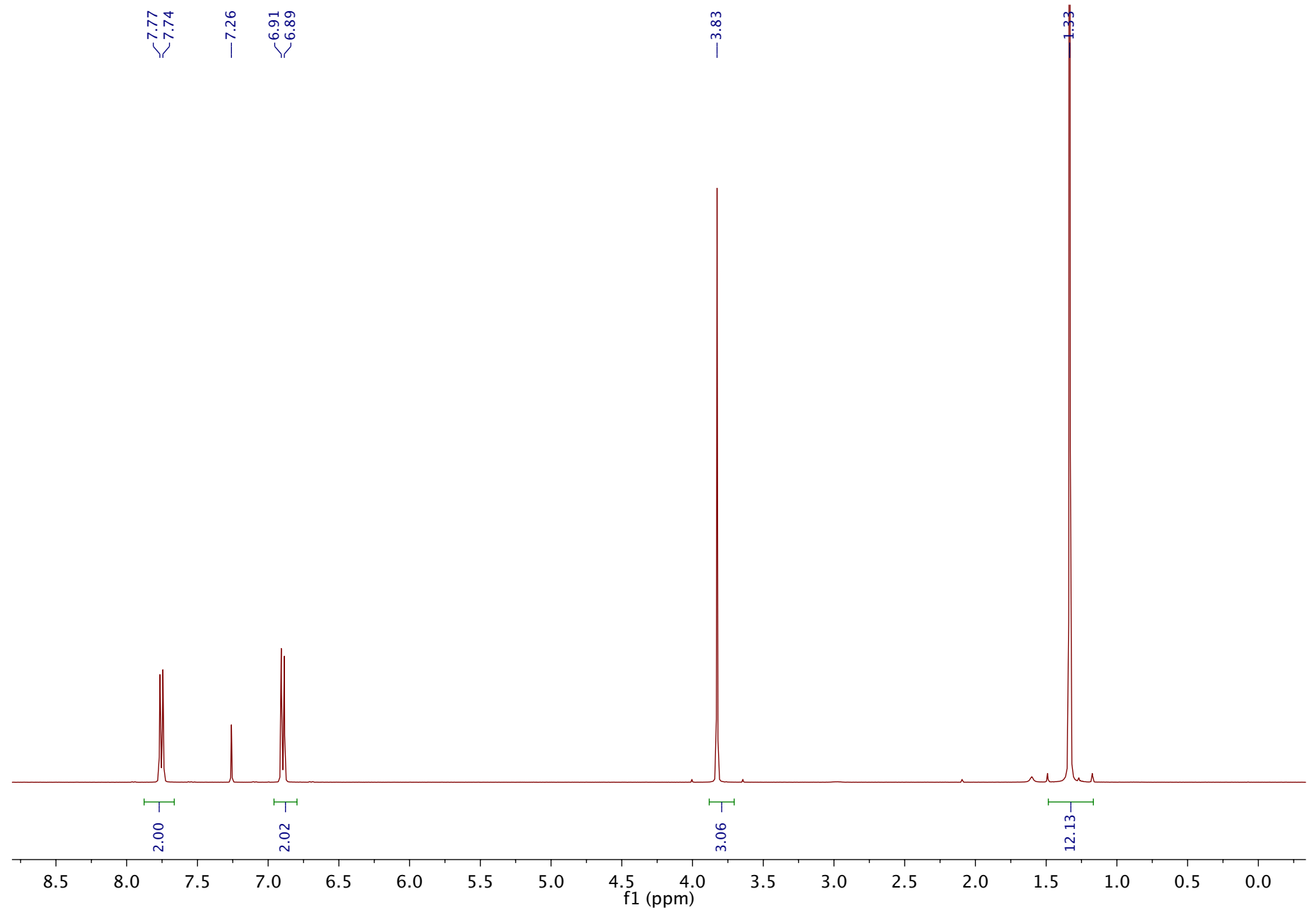




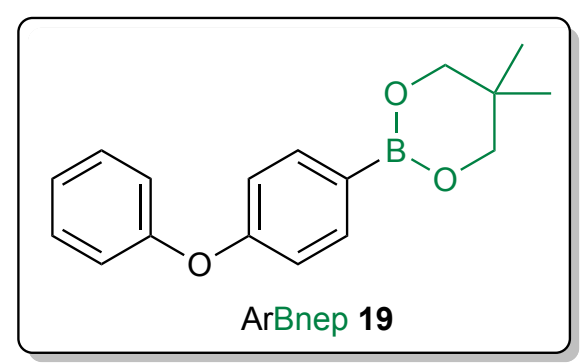

${ }^{1} \mathrm{H}$ NMR

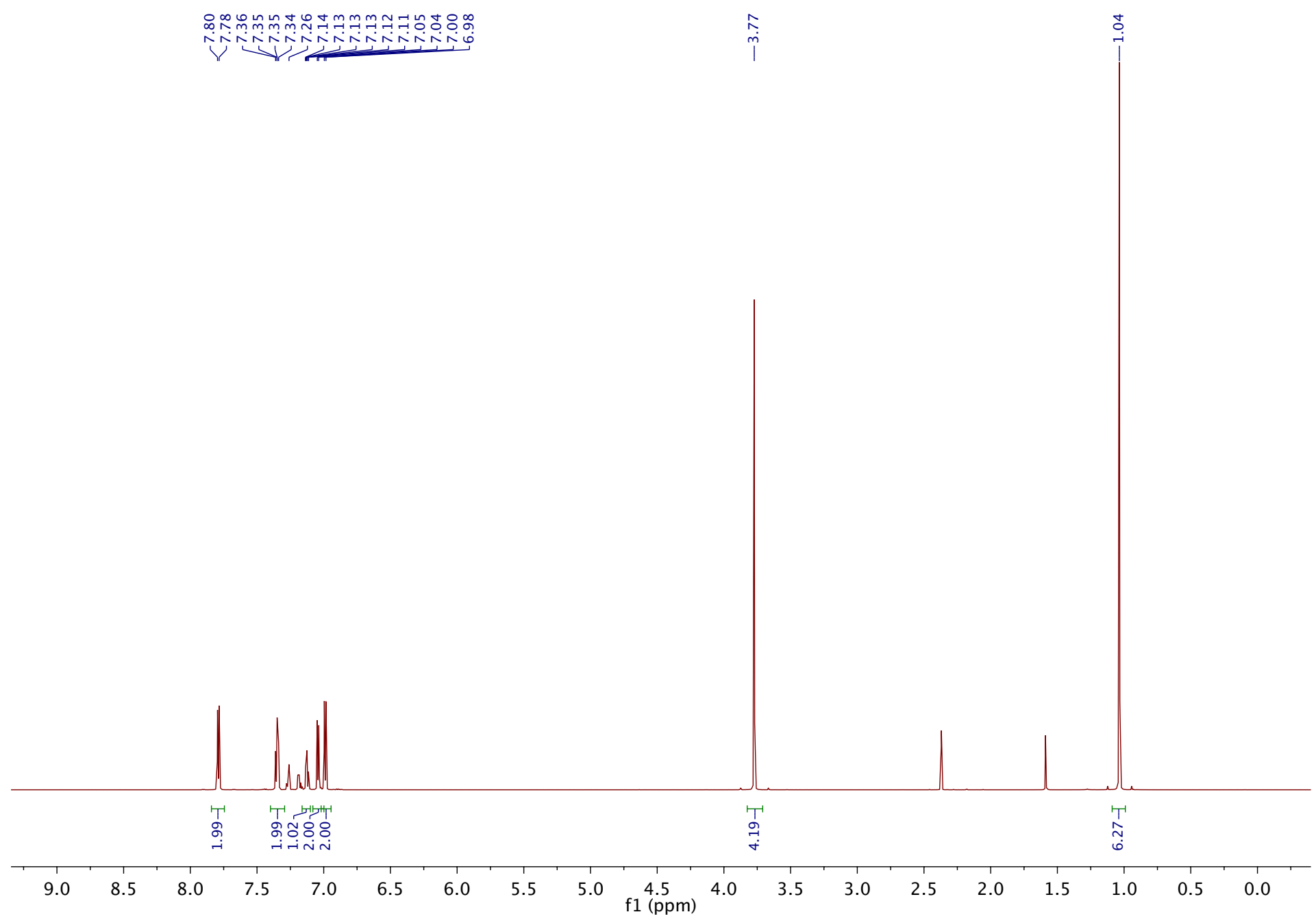




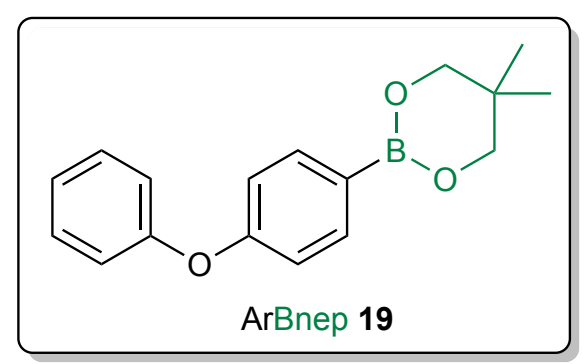

${ }^{13} \mathrm{C} \mathrm{NMR}$

\begin{tabular}{|c|c|c|c|c|}
\hline 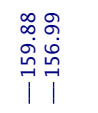 & 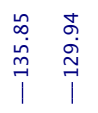 & 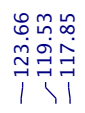 & 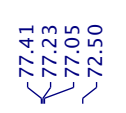 & 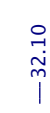 \\
\hline
\end{tabular}

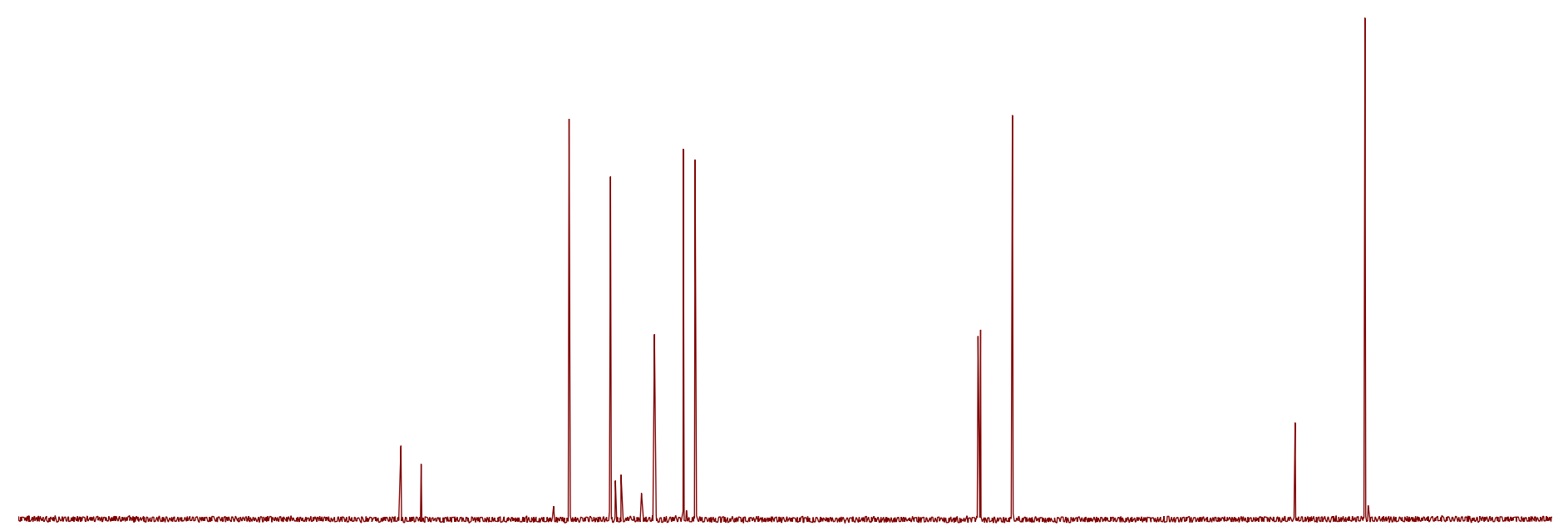




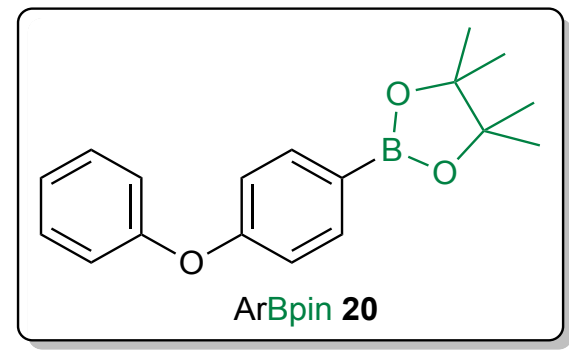

${ }^{1} \mathrm{H}$ NMR

뭉ํำm

inininininimingo

THNR

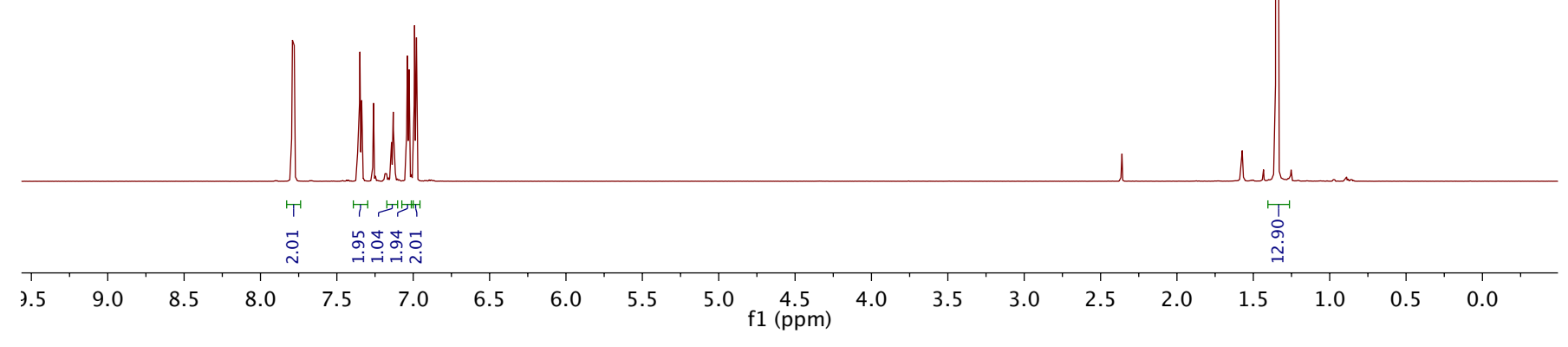




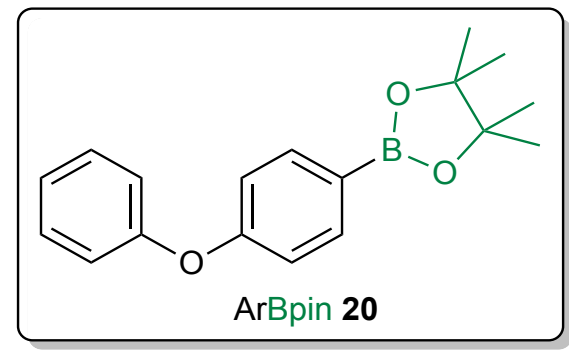

${ }^{13} \mathrm{C}$ NMR

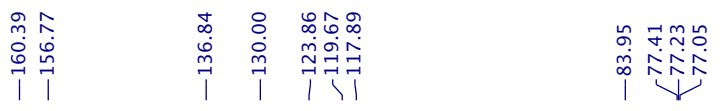

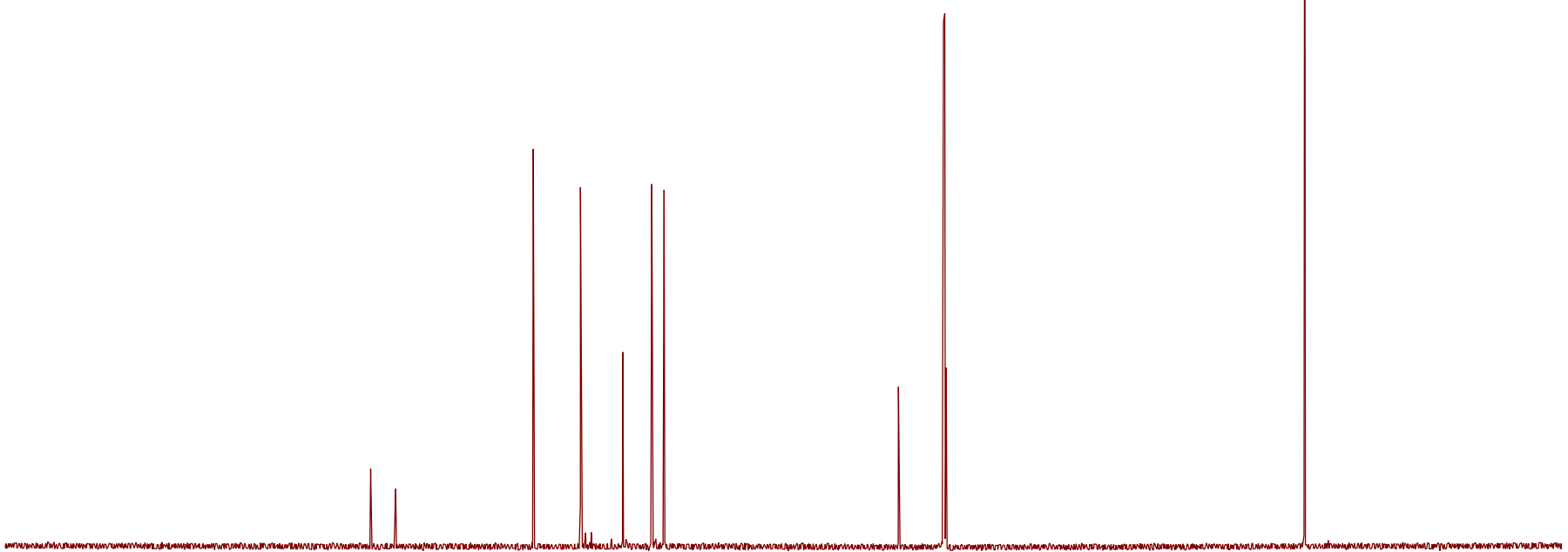

$\begin{array}{lllllllllllllllllllllllll}210 & 200 & 190 & 180 & 170 & 160 & 150 & 140 & 130 & 120 & 110 & 100 & 90 & 80 & 70 & 60 & 50 & 40 & 30 & 20 & 10 & 0 & -10\end{array}$ 


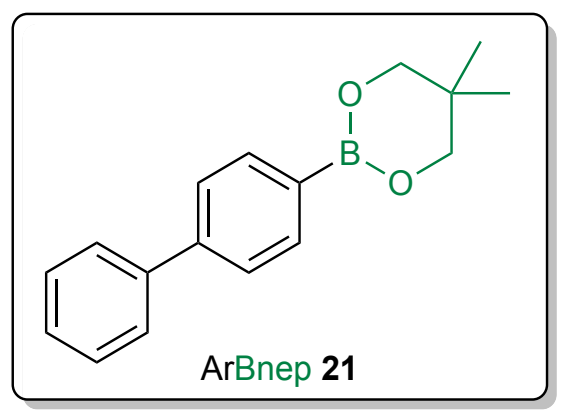

${ }^{1} \mathrm{H}$ NMR

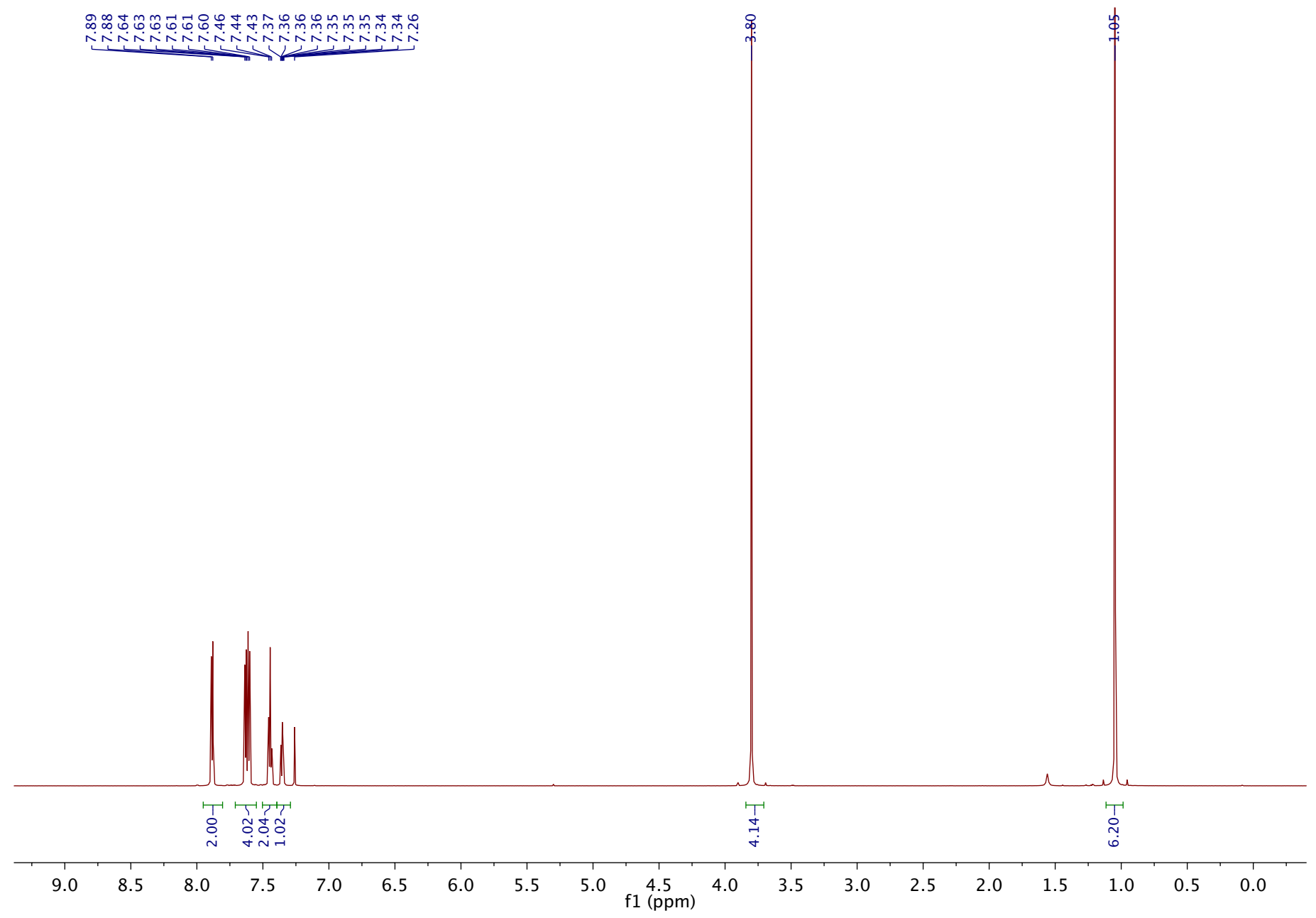




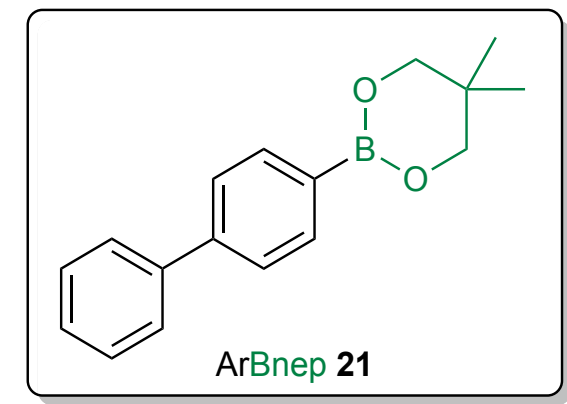

${ }^{13} \mathrm{C}$ NMR

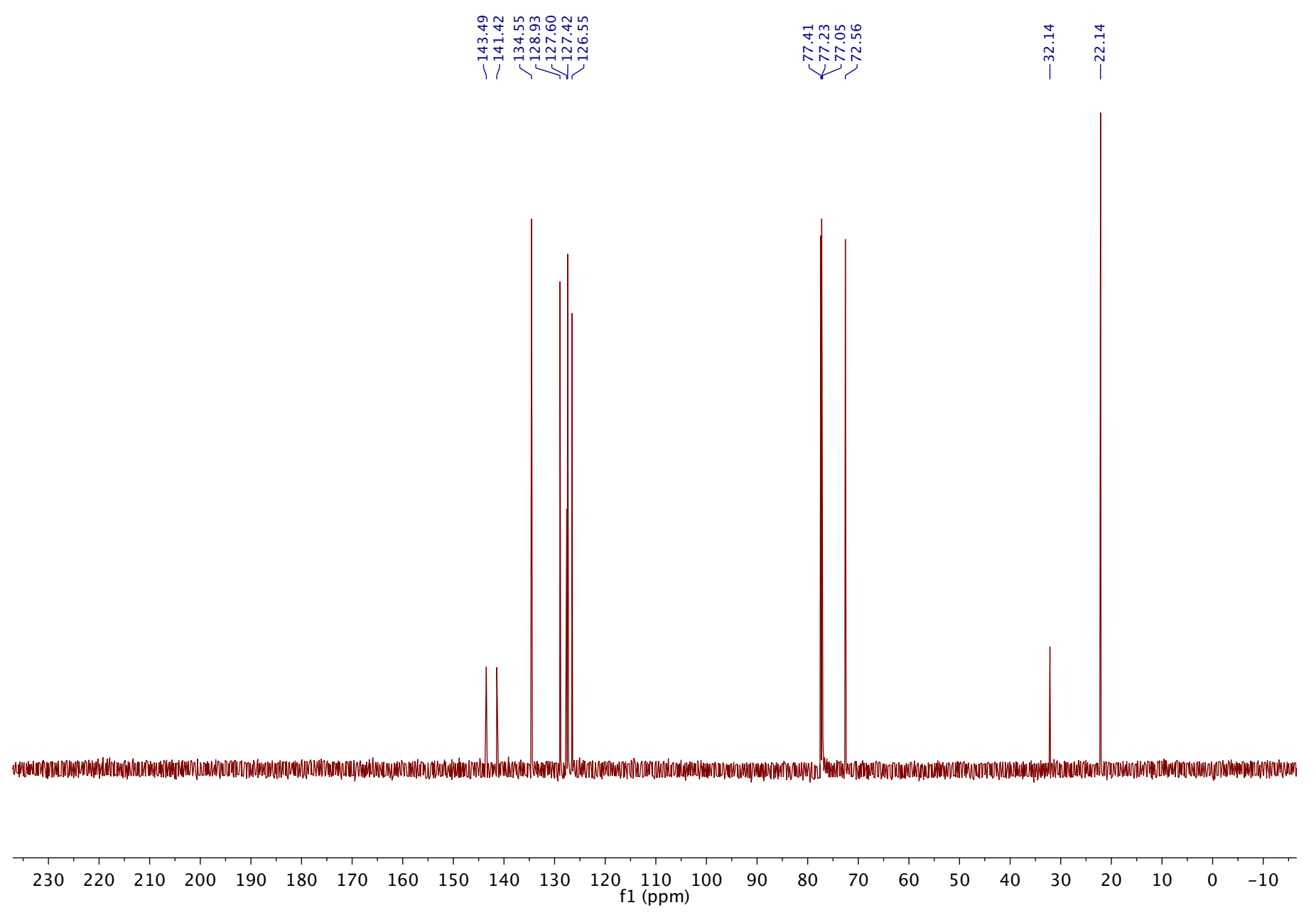




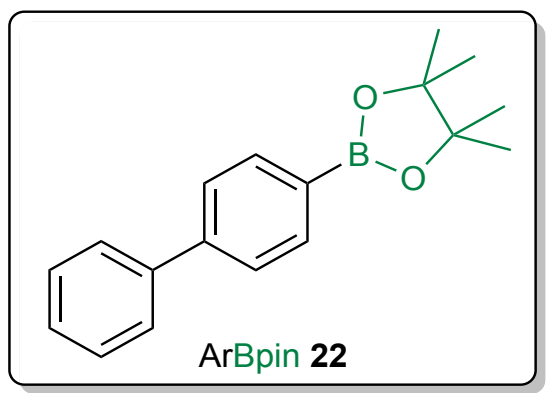

${ }^{1} \mathrm{H}$ NMR

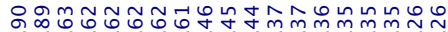

Nininininiminitin

(H)

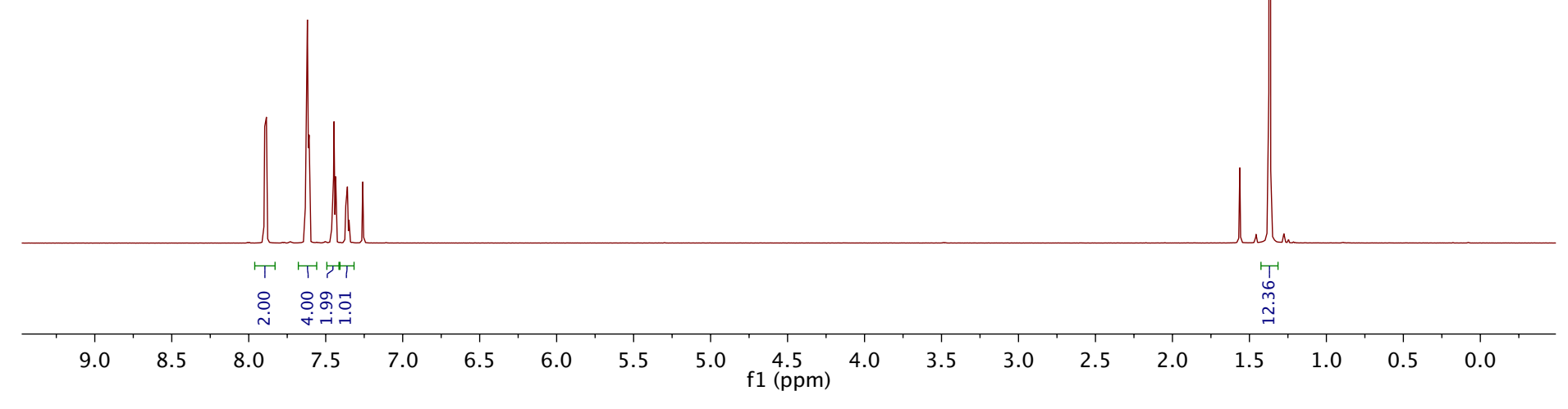




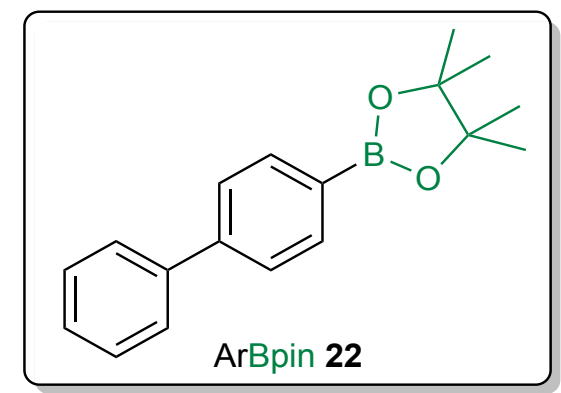

${ }^{13} \mathrm{C}$ NMR
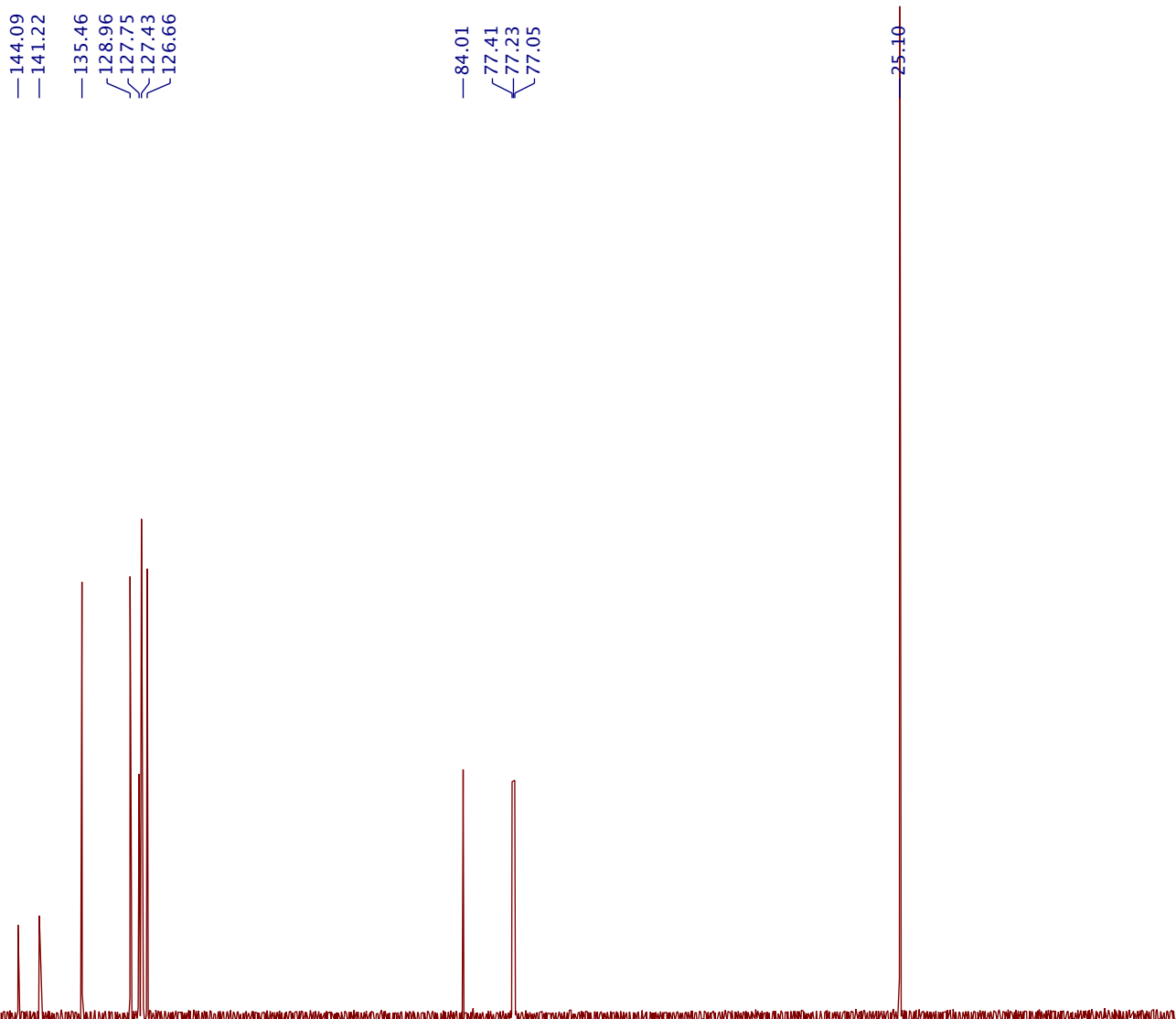

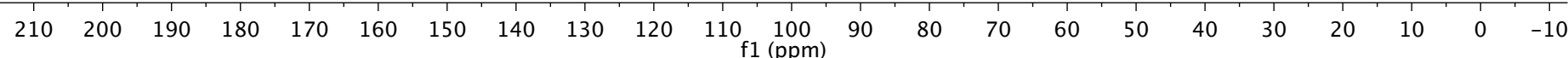




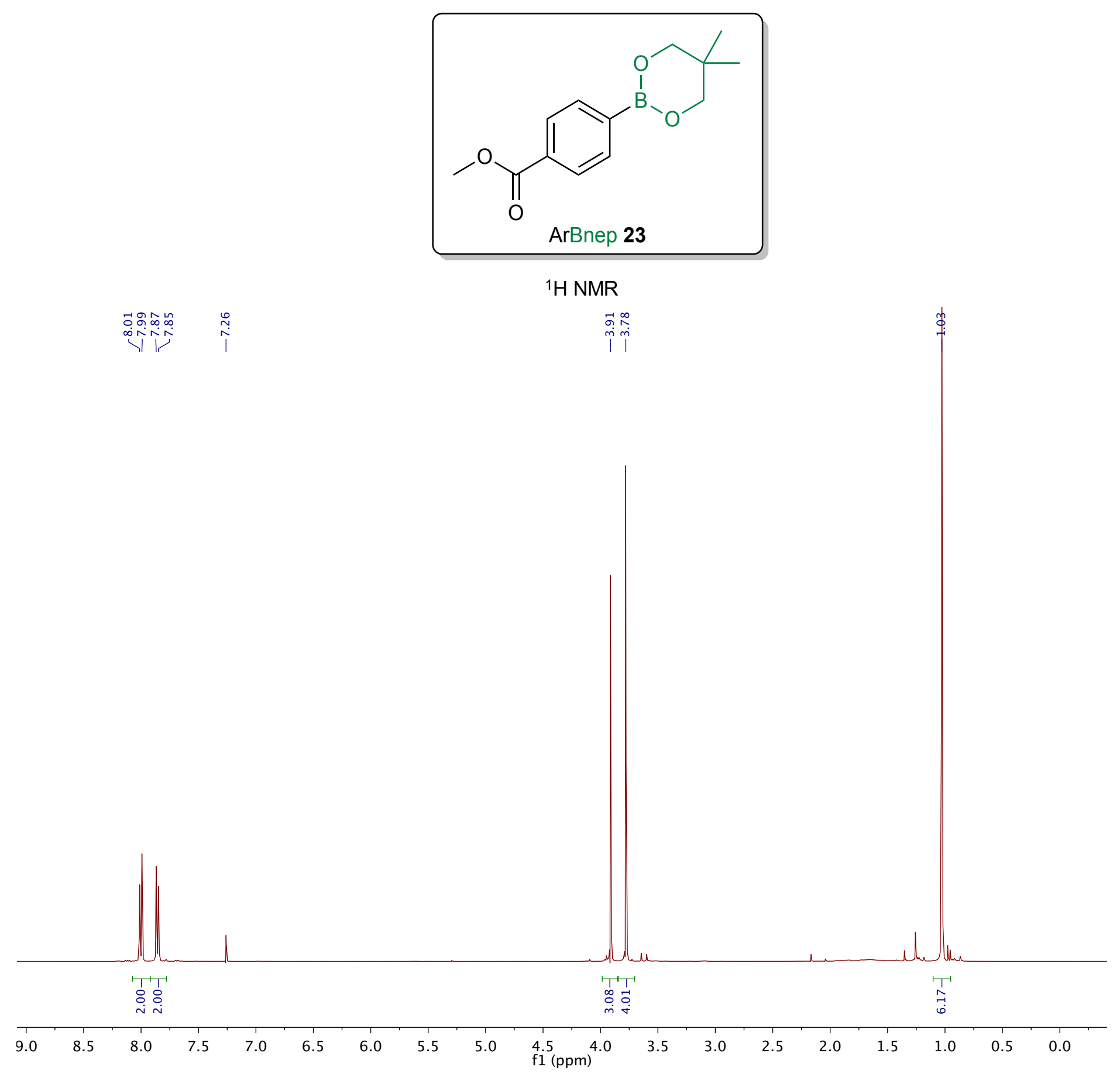




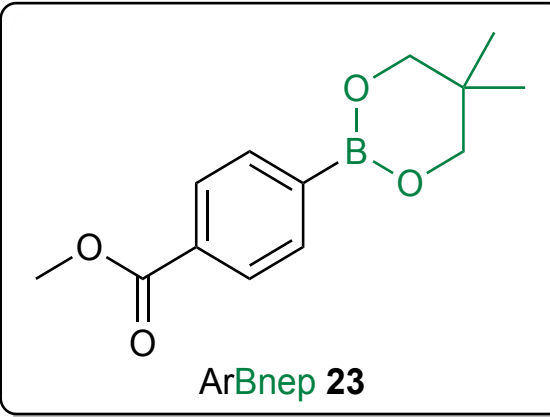

${ }^{13} \mathrm{C}$ NMR

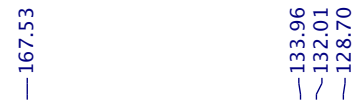

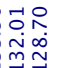

$$
\begin{aligned}
& 117
\end{aligned}
$$

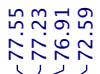

$\stackrel{\infty}{\sim}$

$\stackrel{ }{\stackrel{9}{i}} \stackrel{\stackrel{ }{i}}{i}$

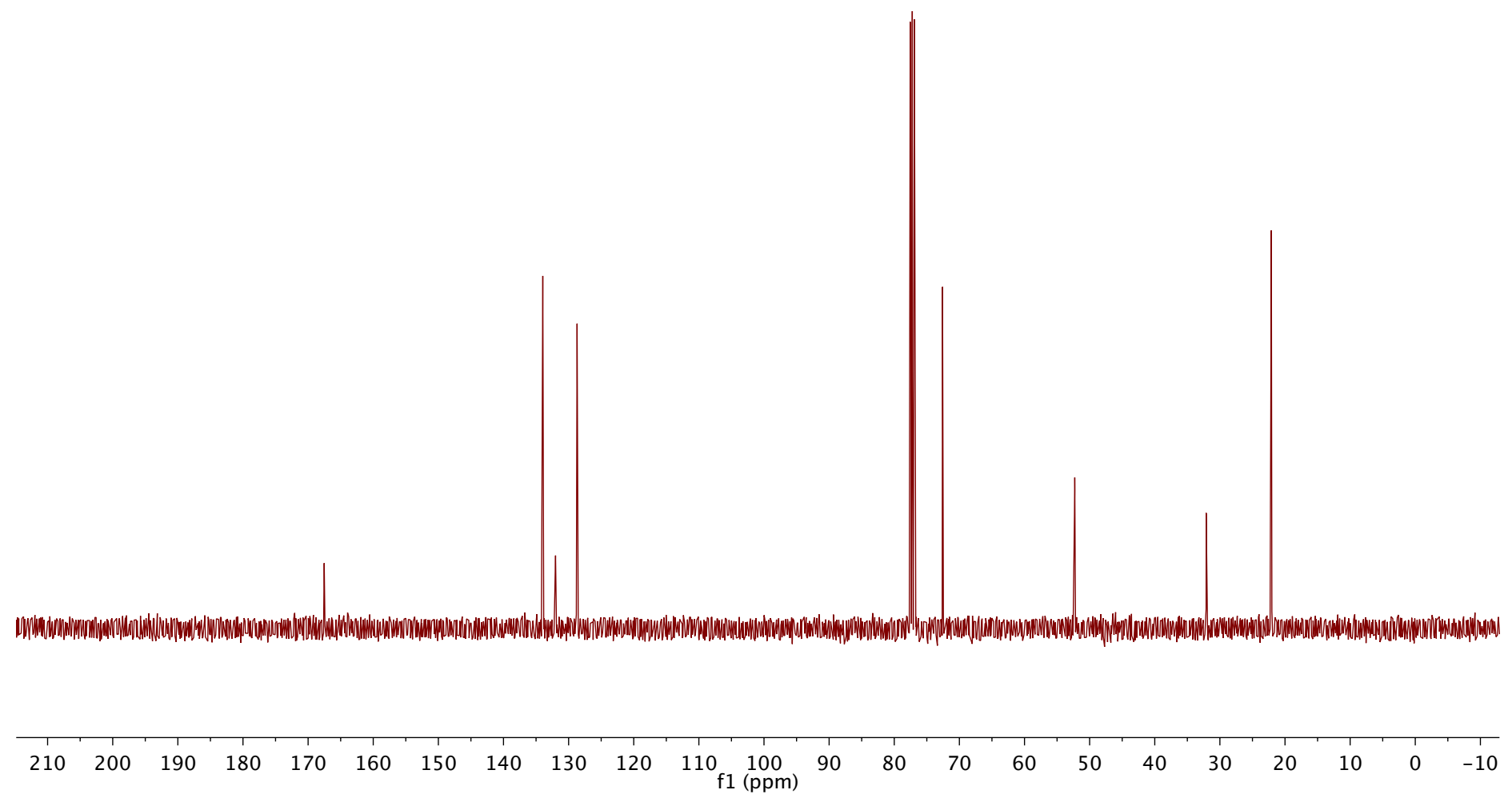




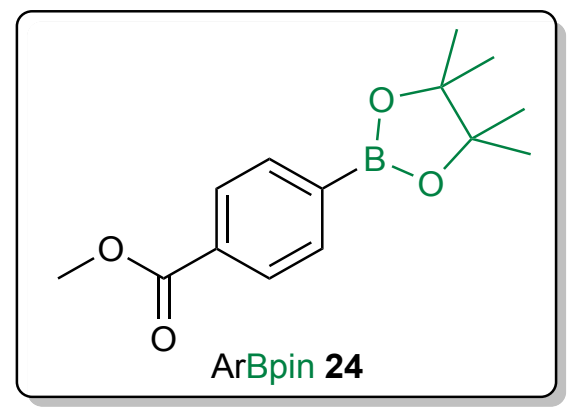

${ }^{1} \mathrm{H}$ NMR

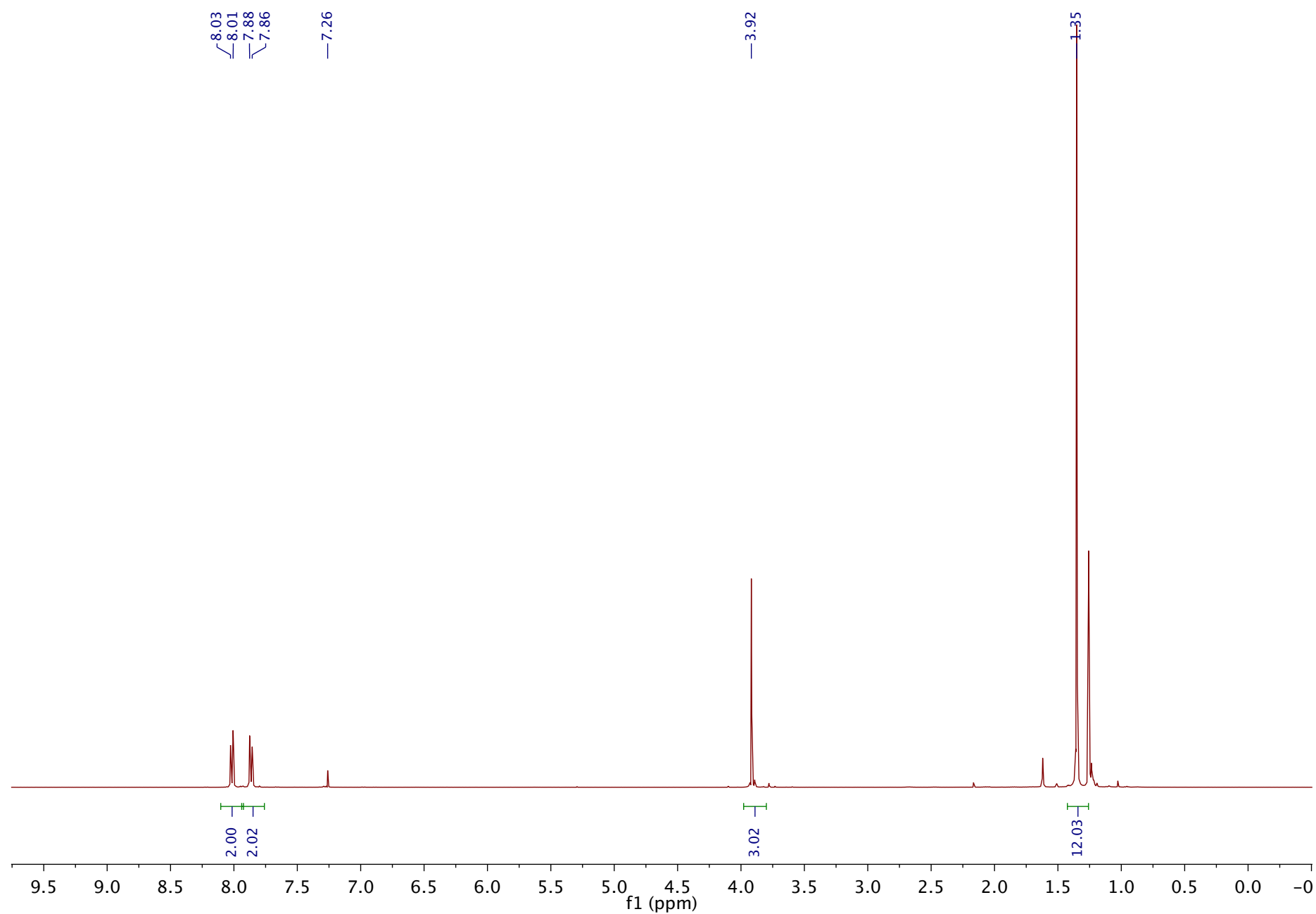




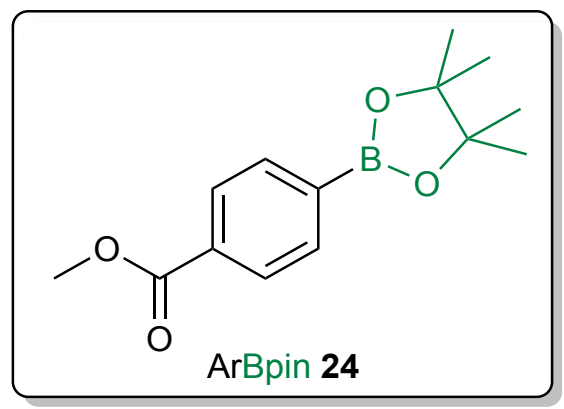

${ }^{13} \mathrm{C}$ NMR
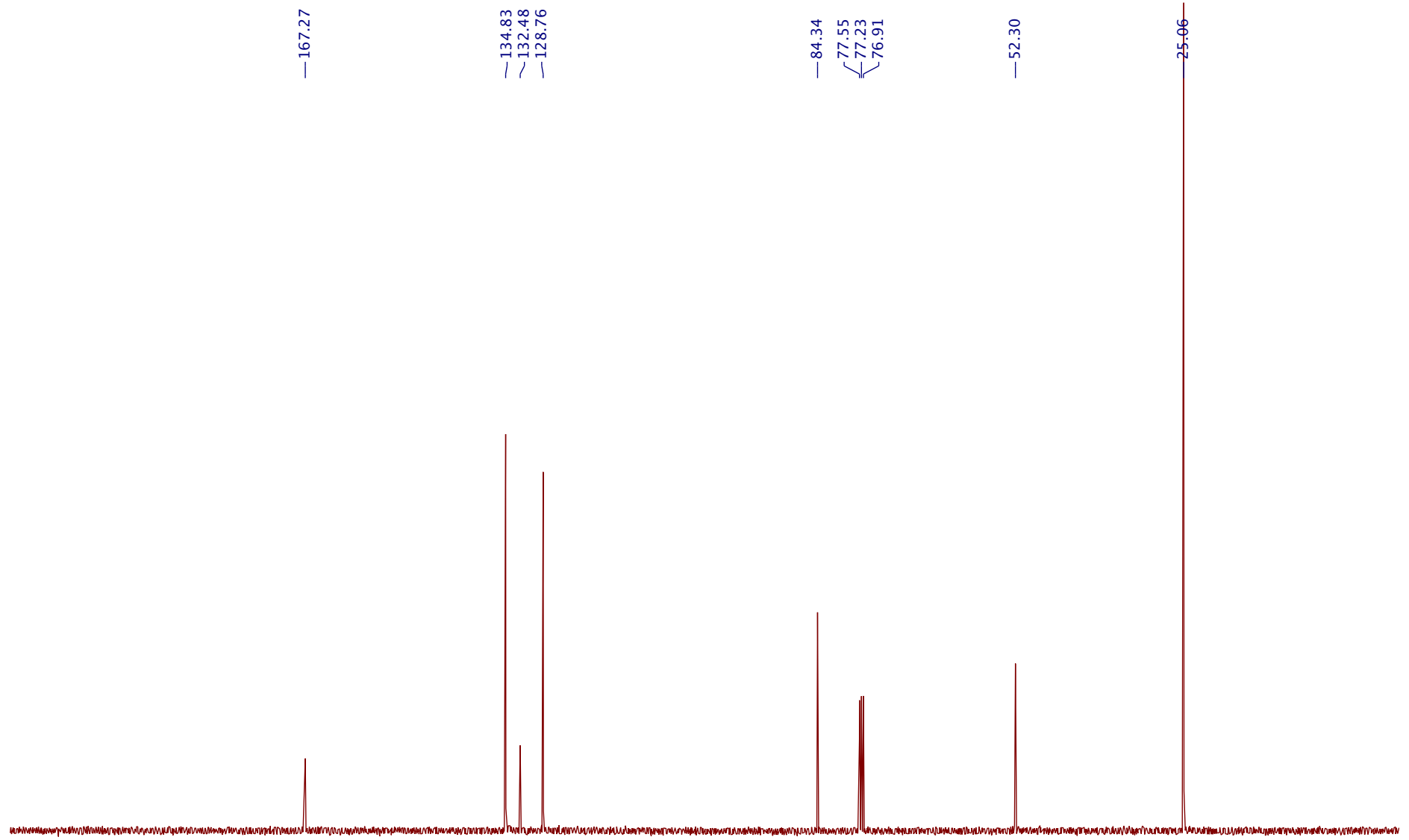


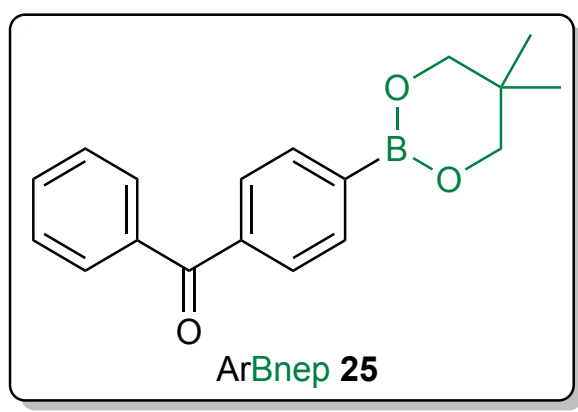

${ }^{1} \mathrm{H}$ NMR

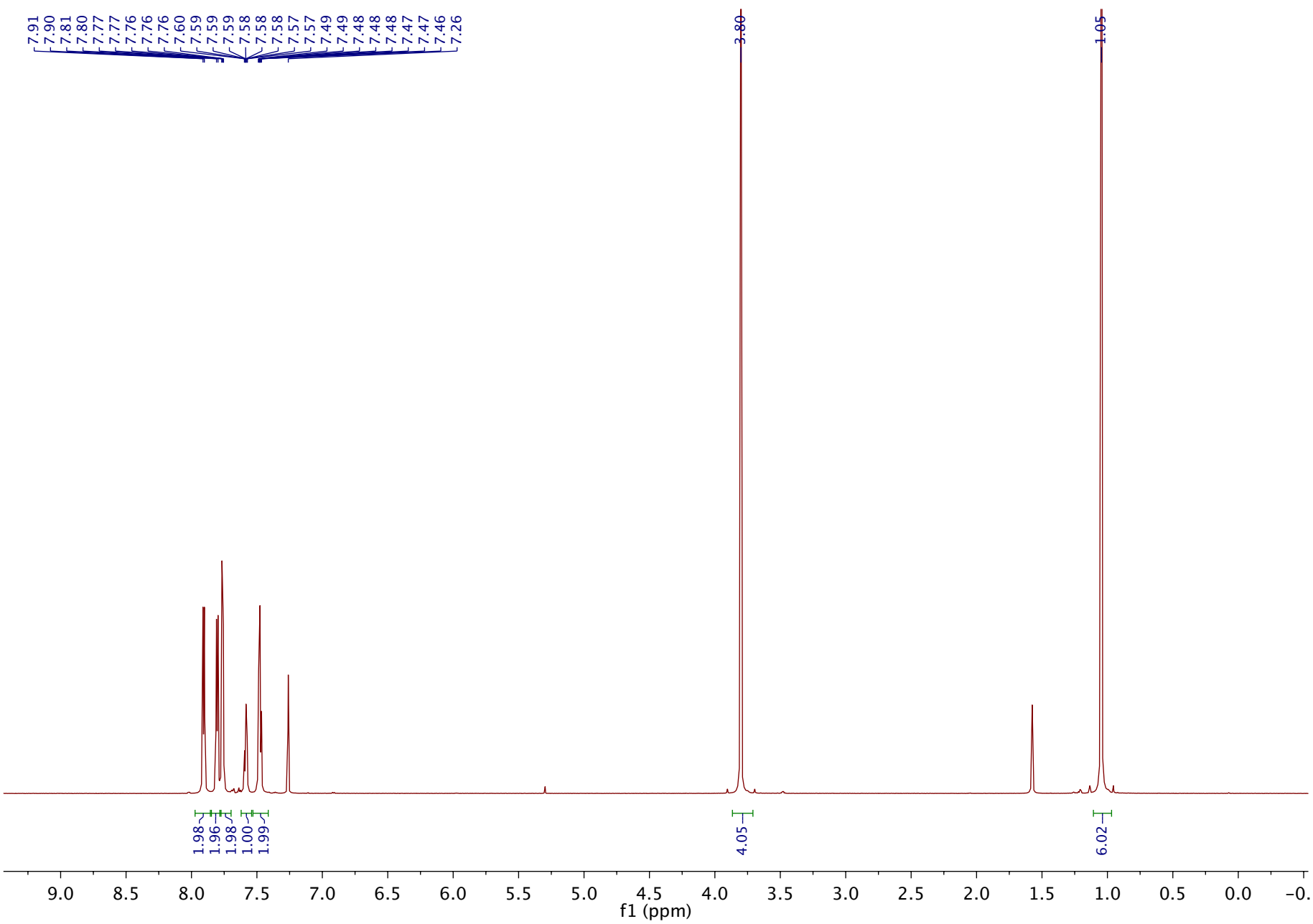




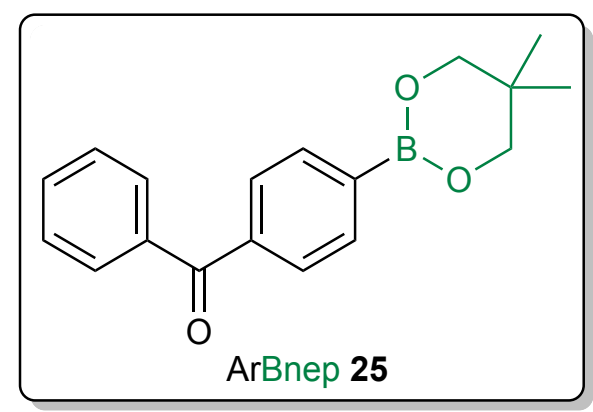

${ }^{13} \mathrm{C}$ NMR

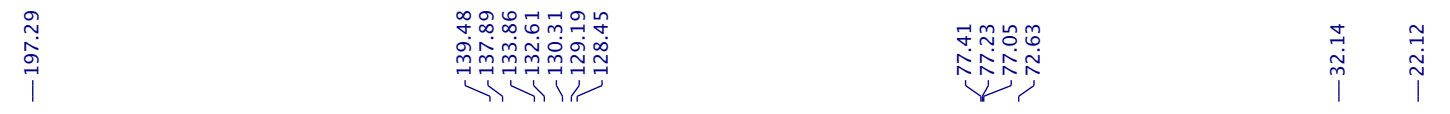

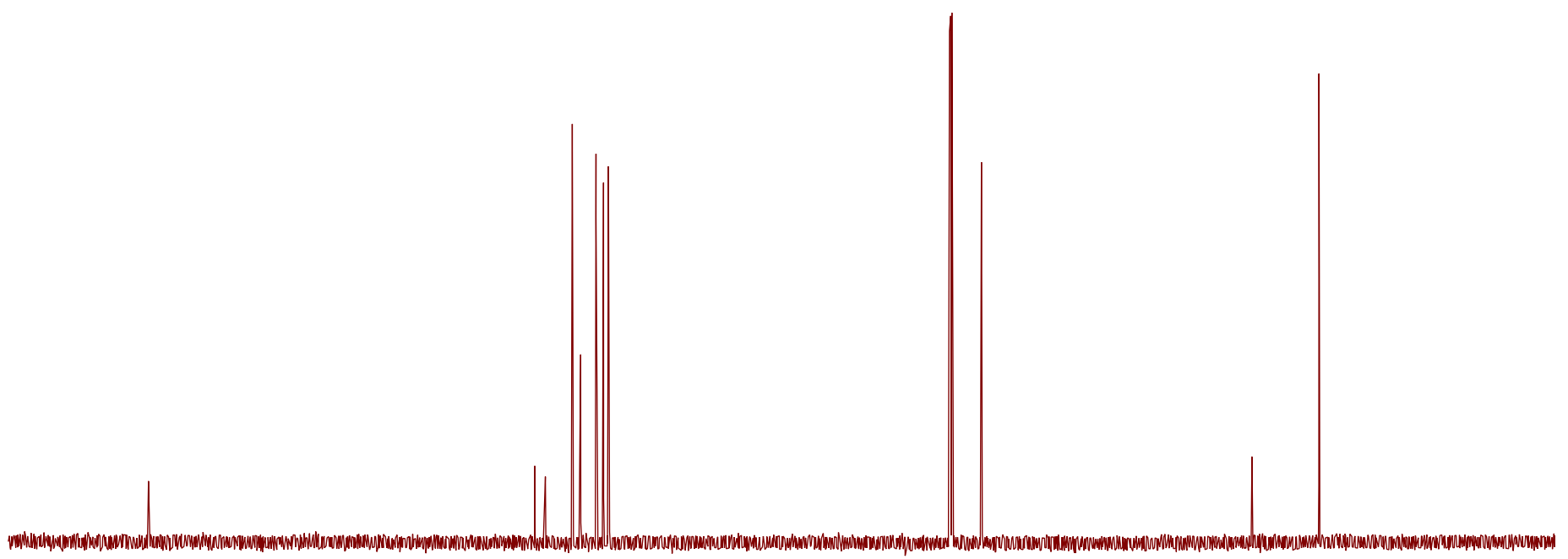

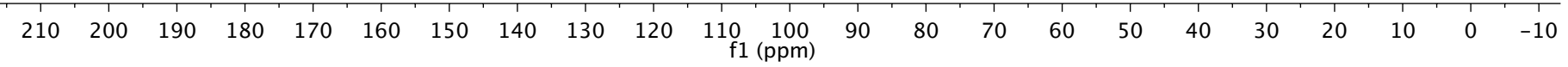




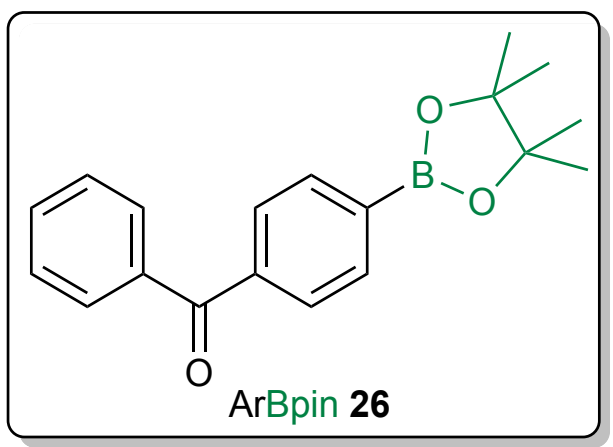

${ }^{1} \mathrm{H}$ NMR

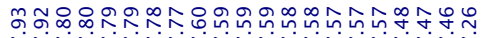

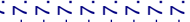

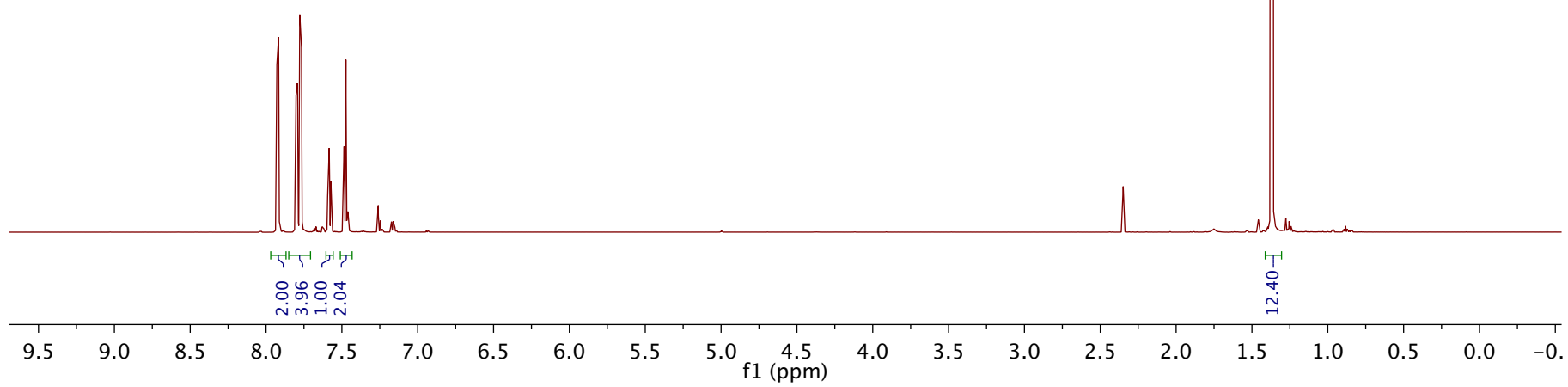




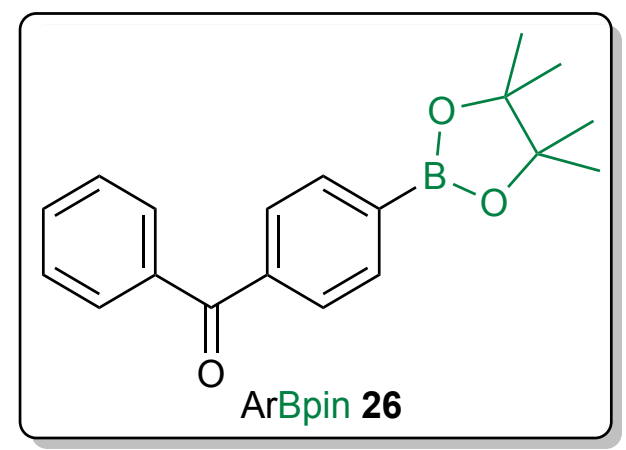

${ }^{13}$ C NMR

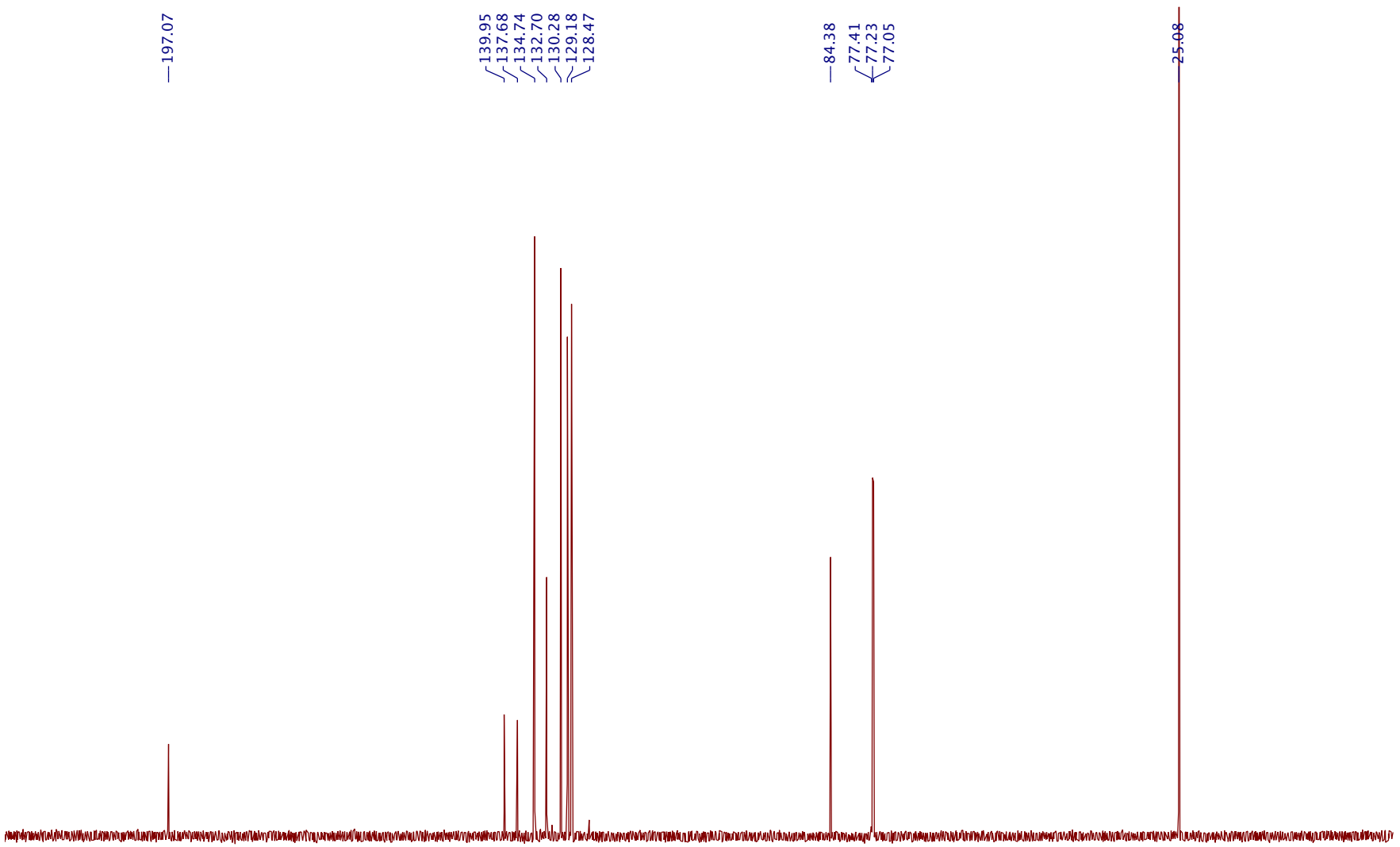

$\begin{array}{llllllllllllllllllllllll}220 & 210 & 200 & 190 & 180 & 170 & 160 & 150 & 140 & 130 & 120 & 110 & 100 & 90 & 80 & 70 & 60 & 50 & 40 & 30 & 20 & 10 & 0 & -11\end{array}$ 


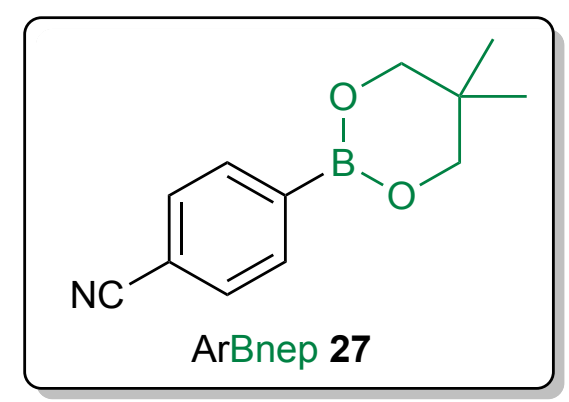

${ }^{1} \mathrm{H}$ NMR

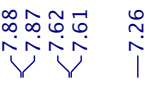

$\stackrel{\infty}{\stackrel{n}{n}}$

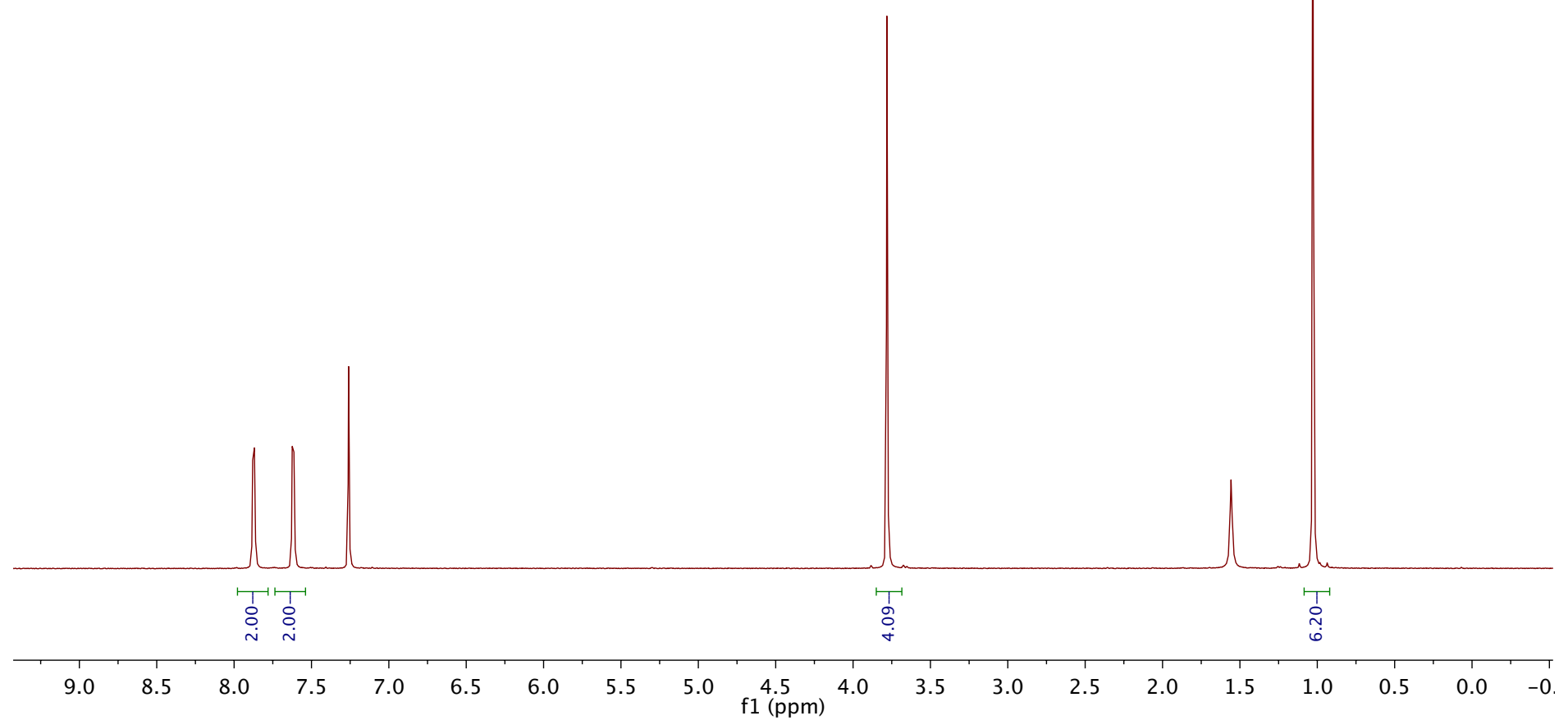



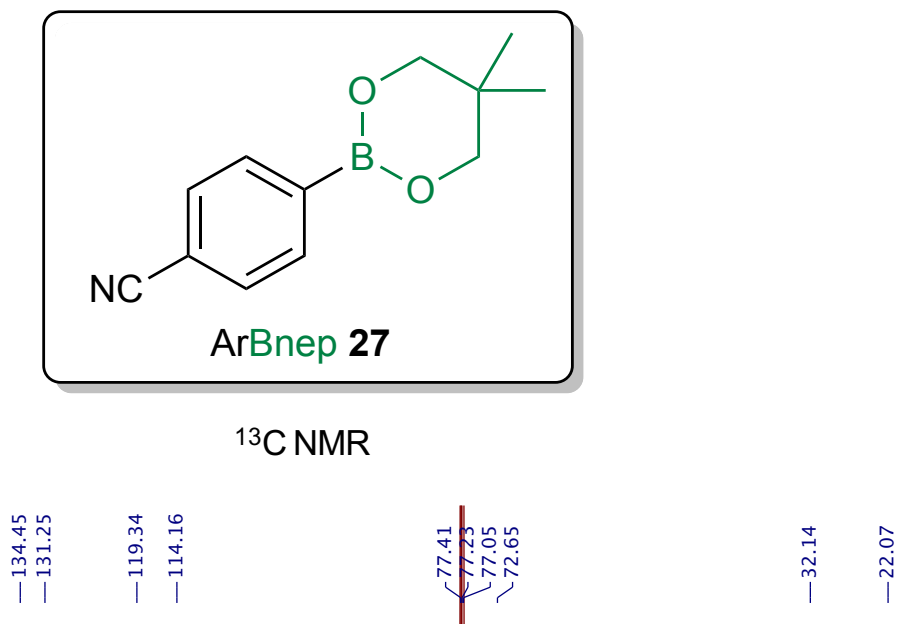

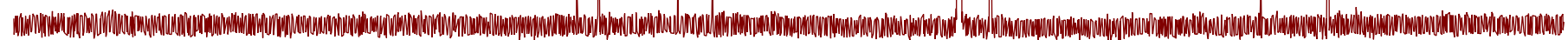

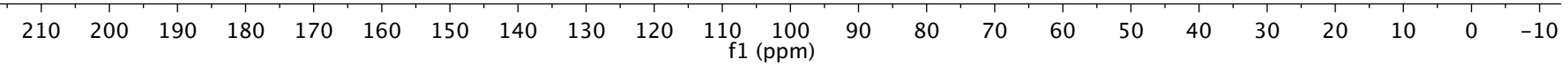




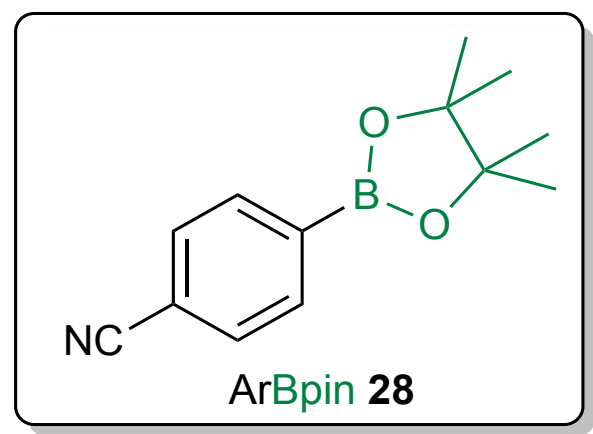

${ }^{1} \mathrm{H}$ NMR

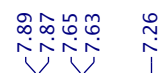

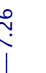
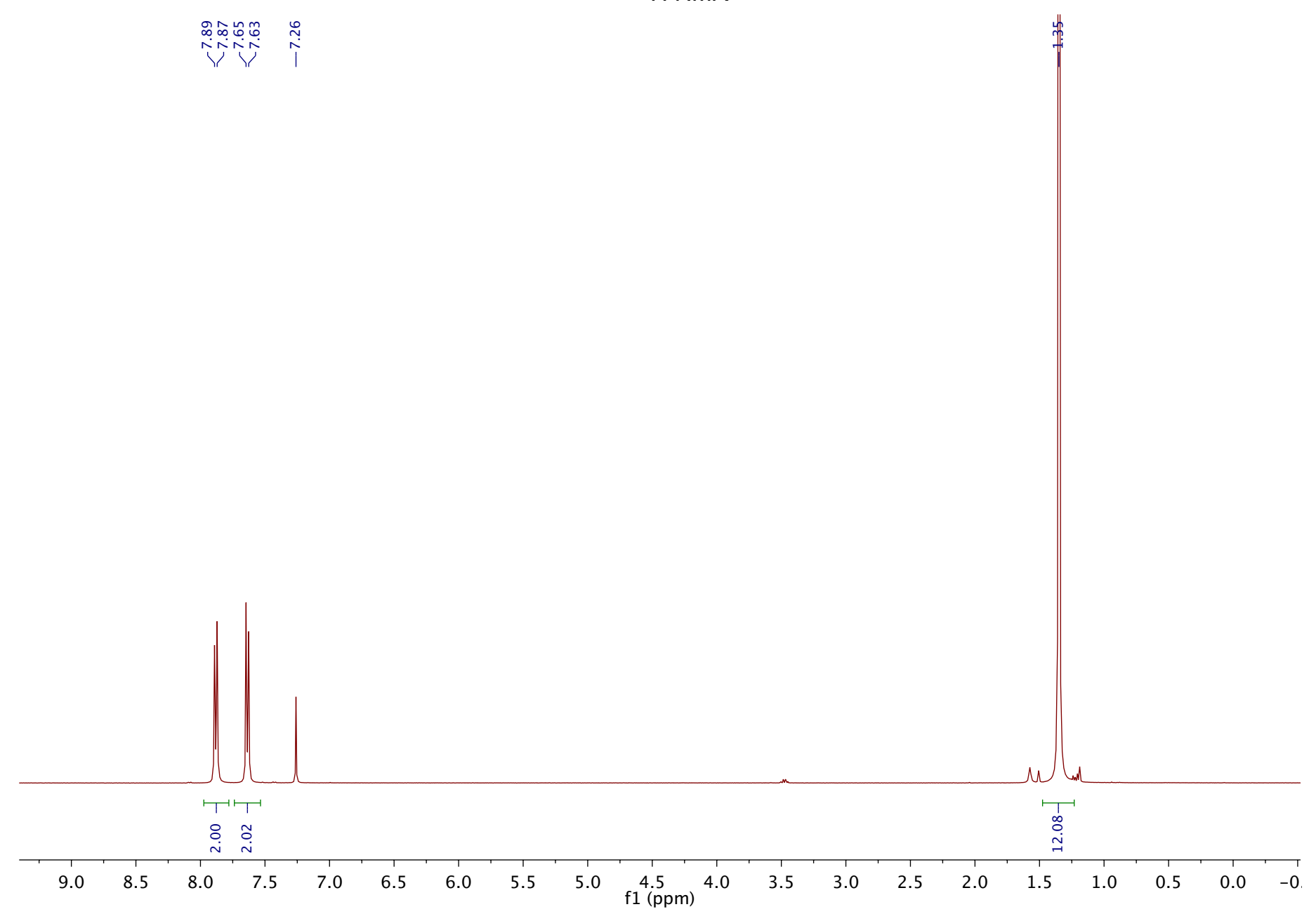


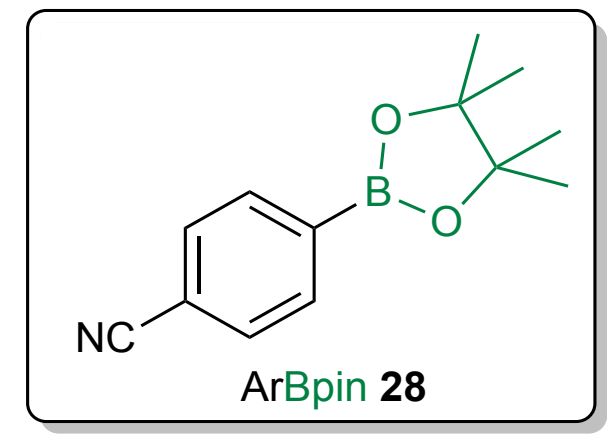

${ }^{13} \mathrm{C}$ NMR
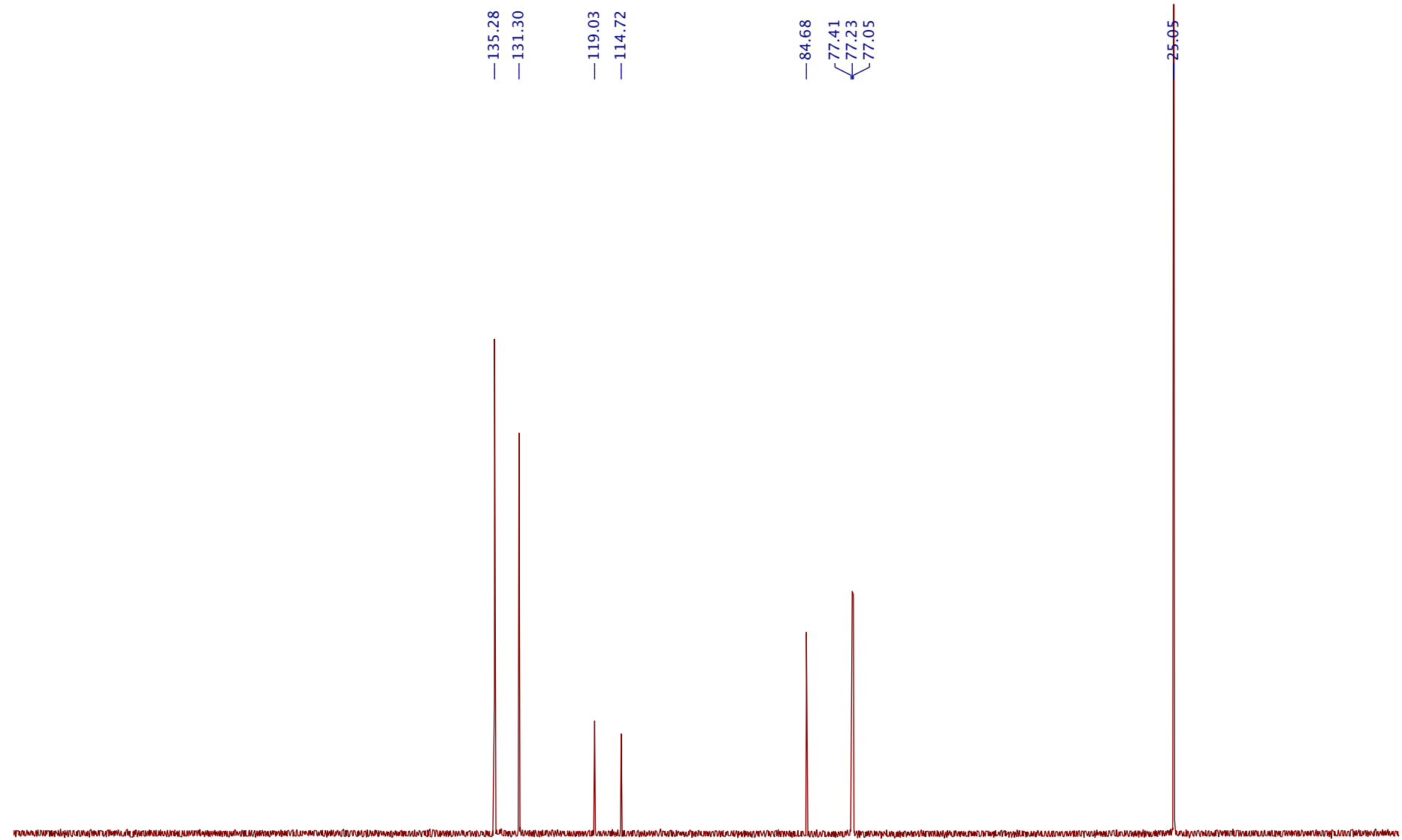


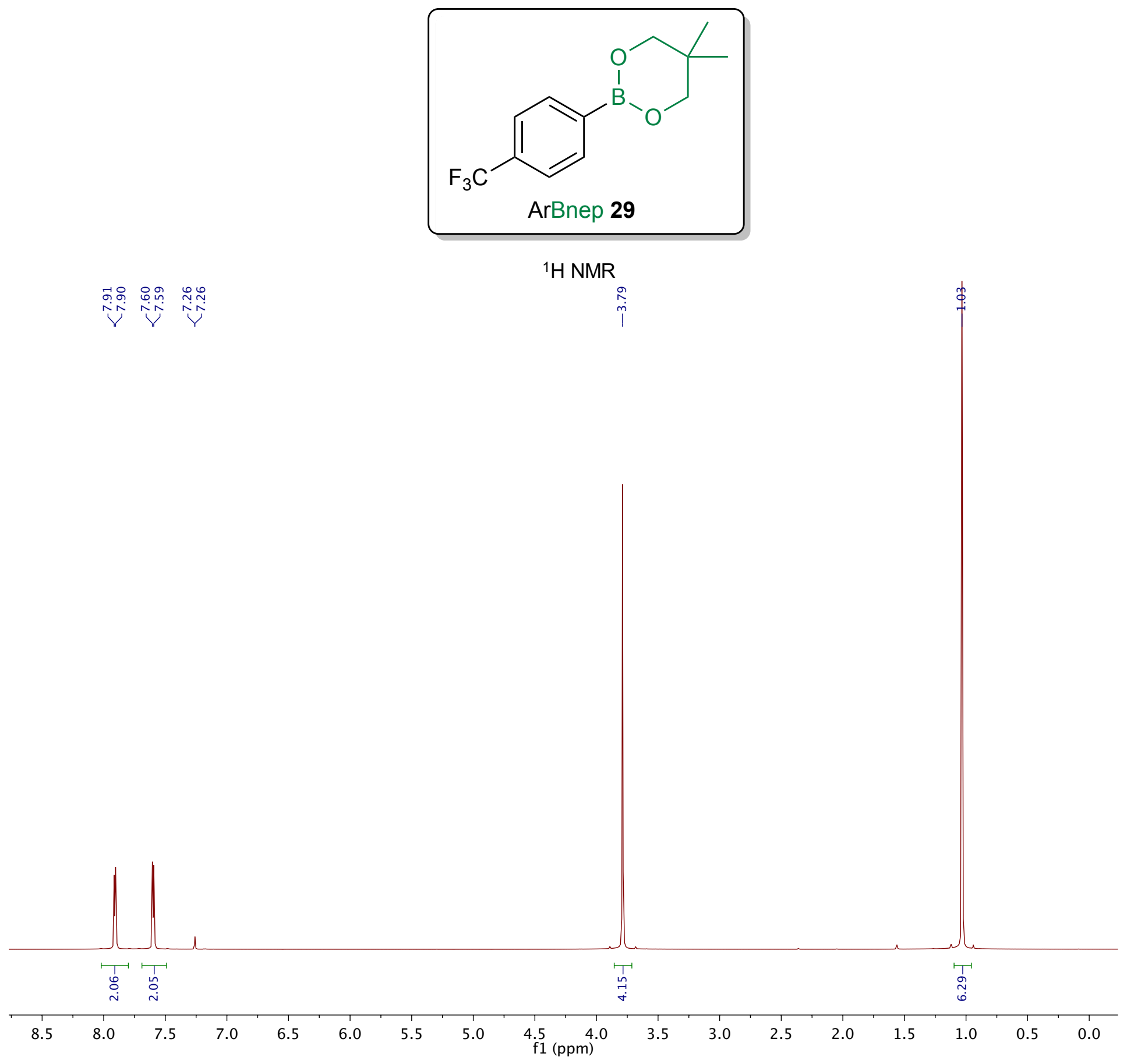




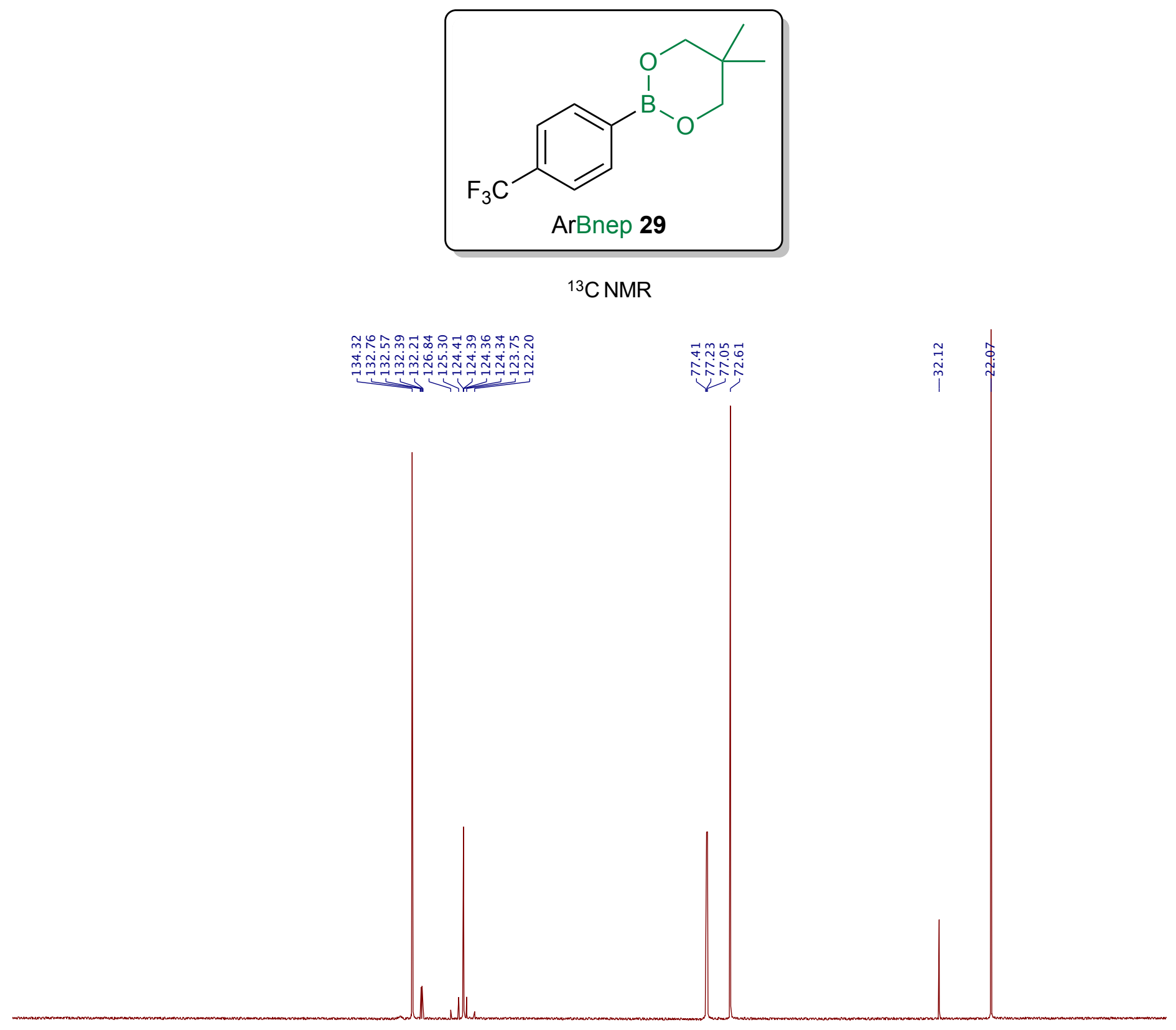

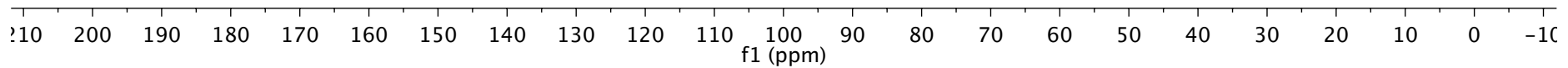




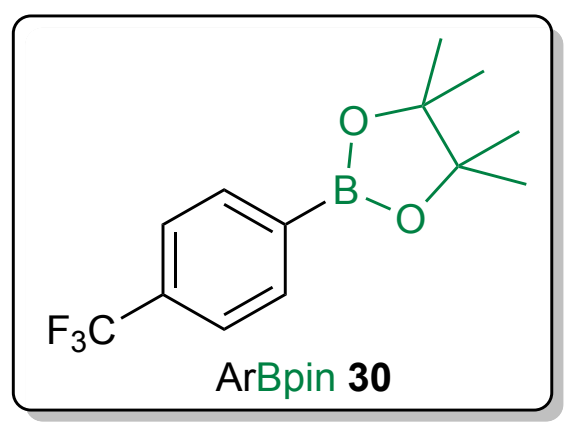

${ }^{1} \mathrm{H}$ NMR
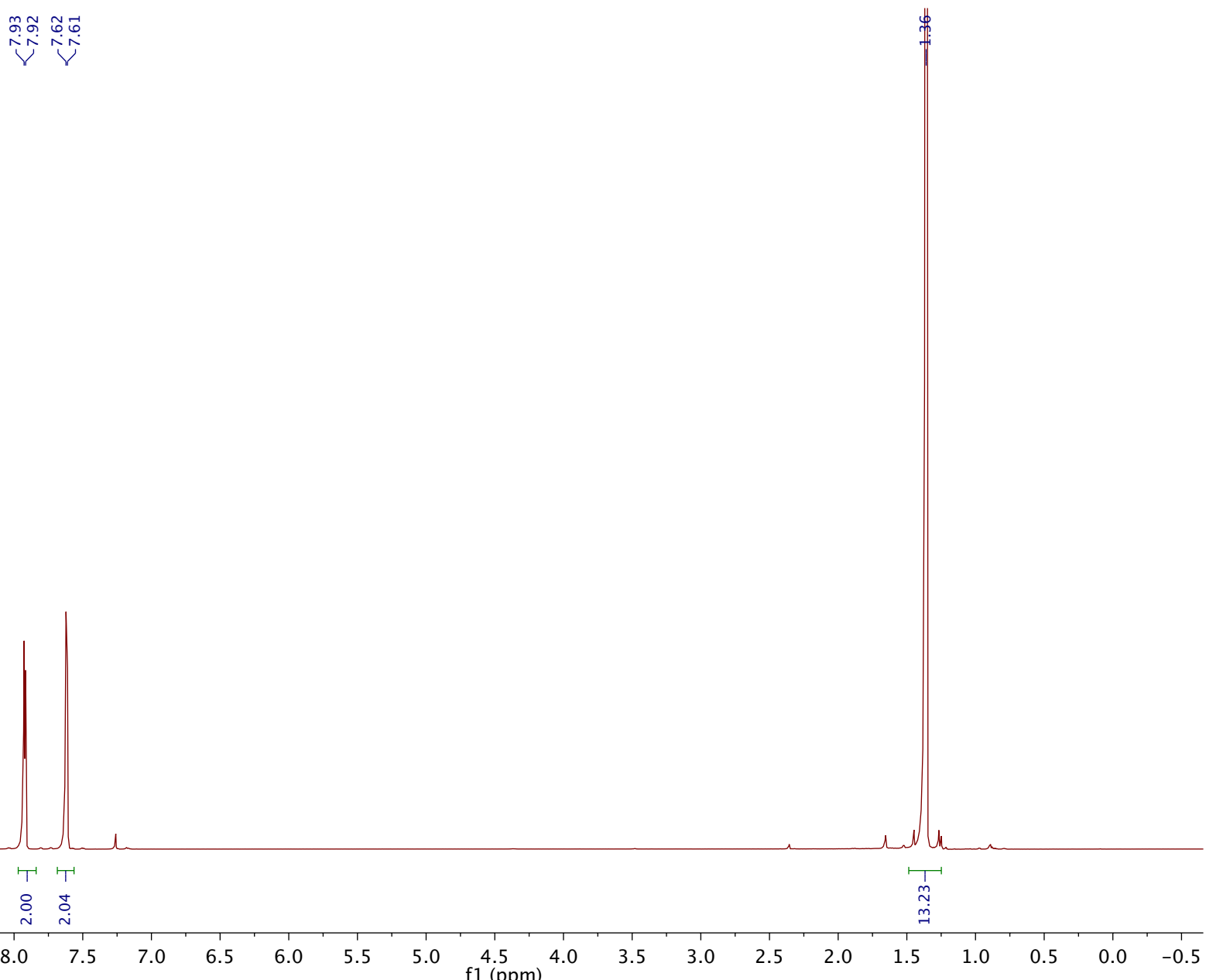


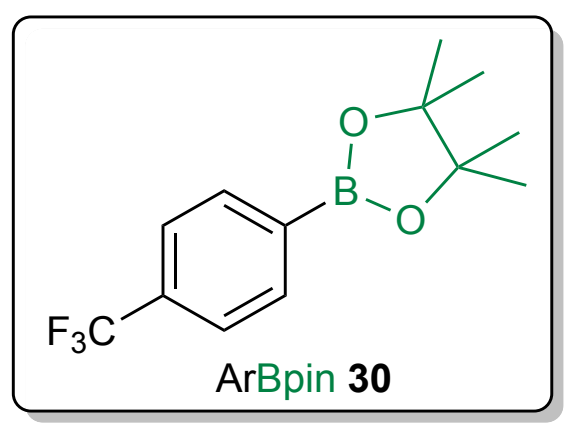

${ }^{13} \mathrm{C}$ NMR

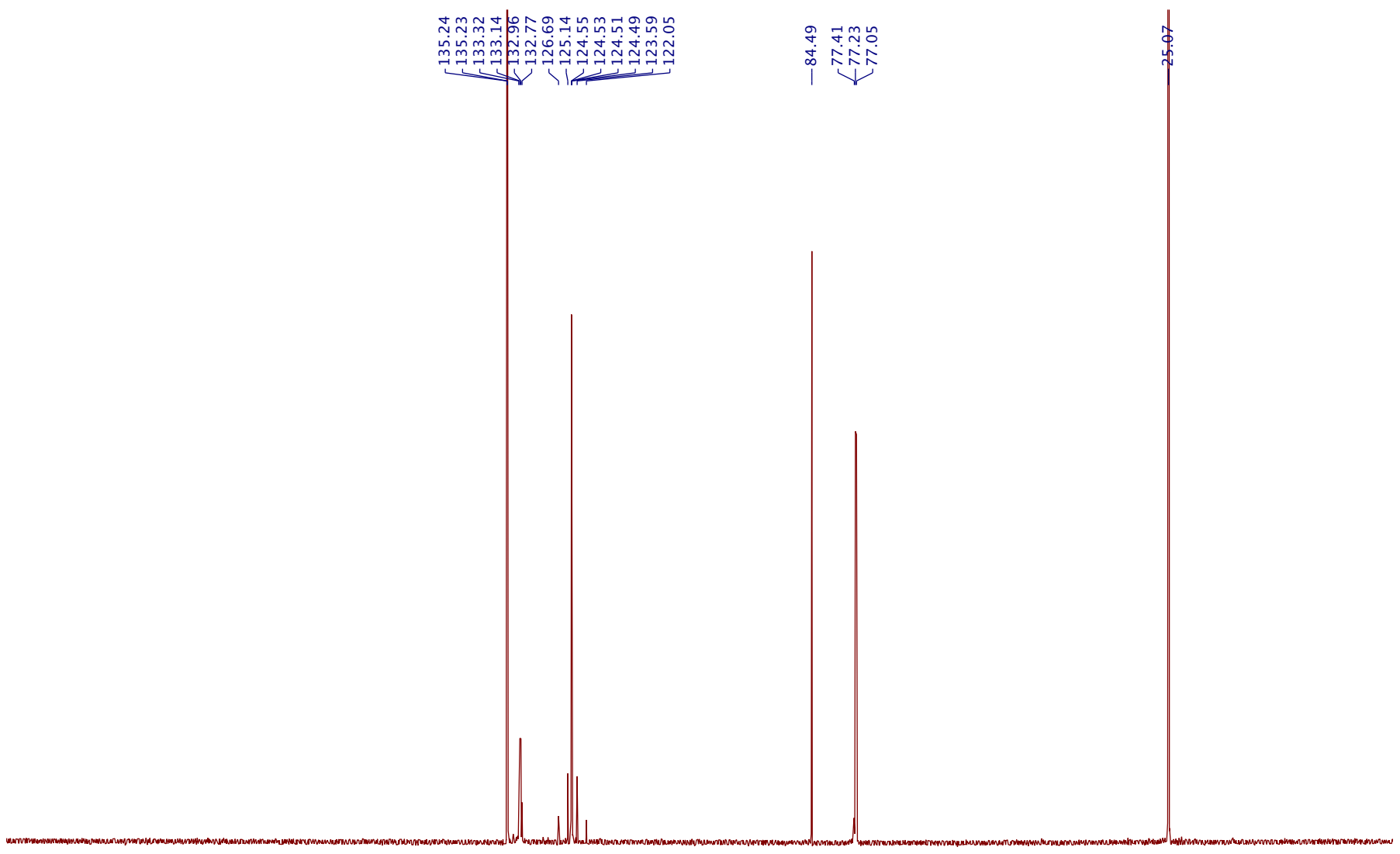

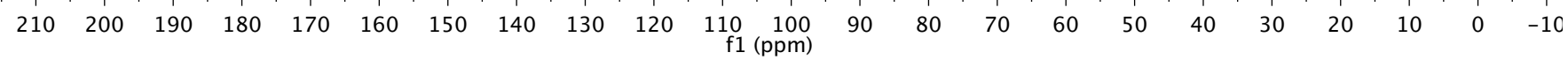




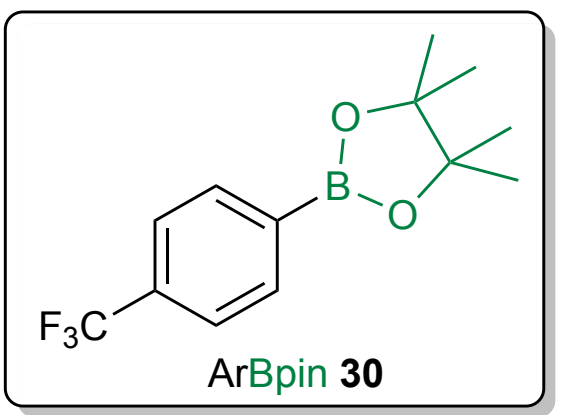

${ }^{19} \mathrm{~F} \mathrm{NMR}$

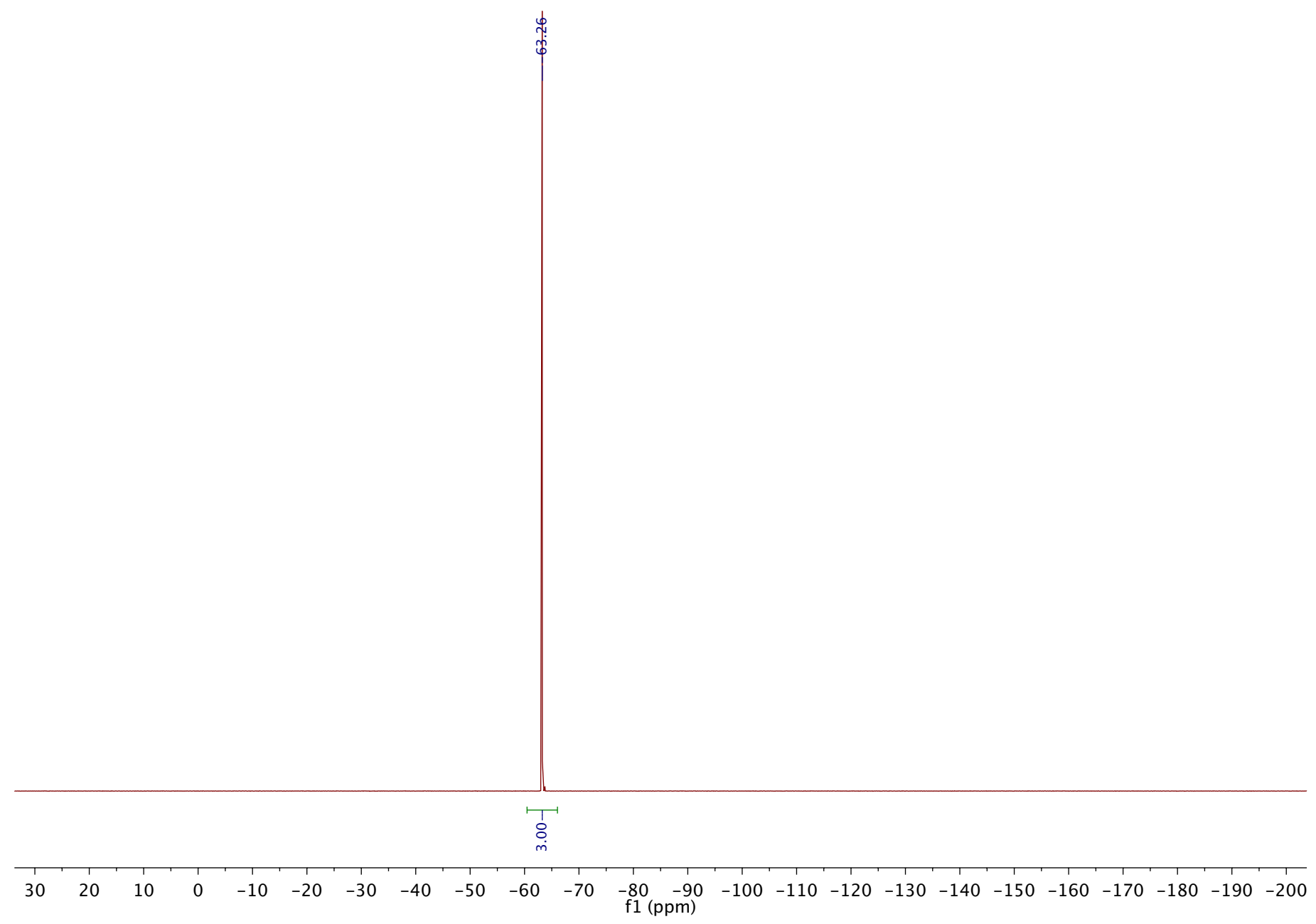




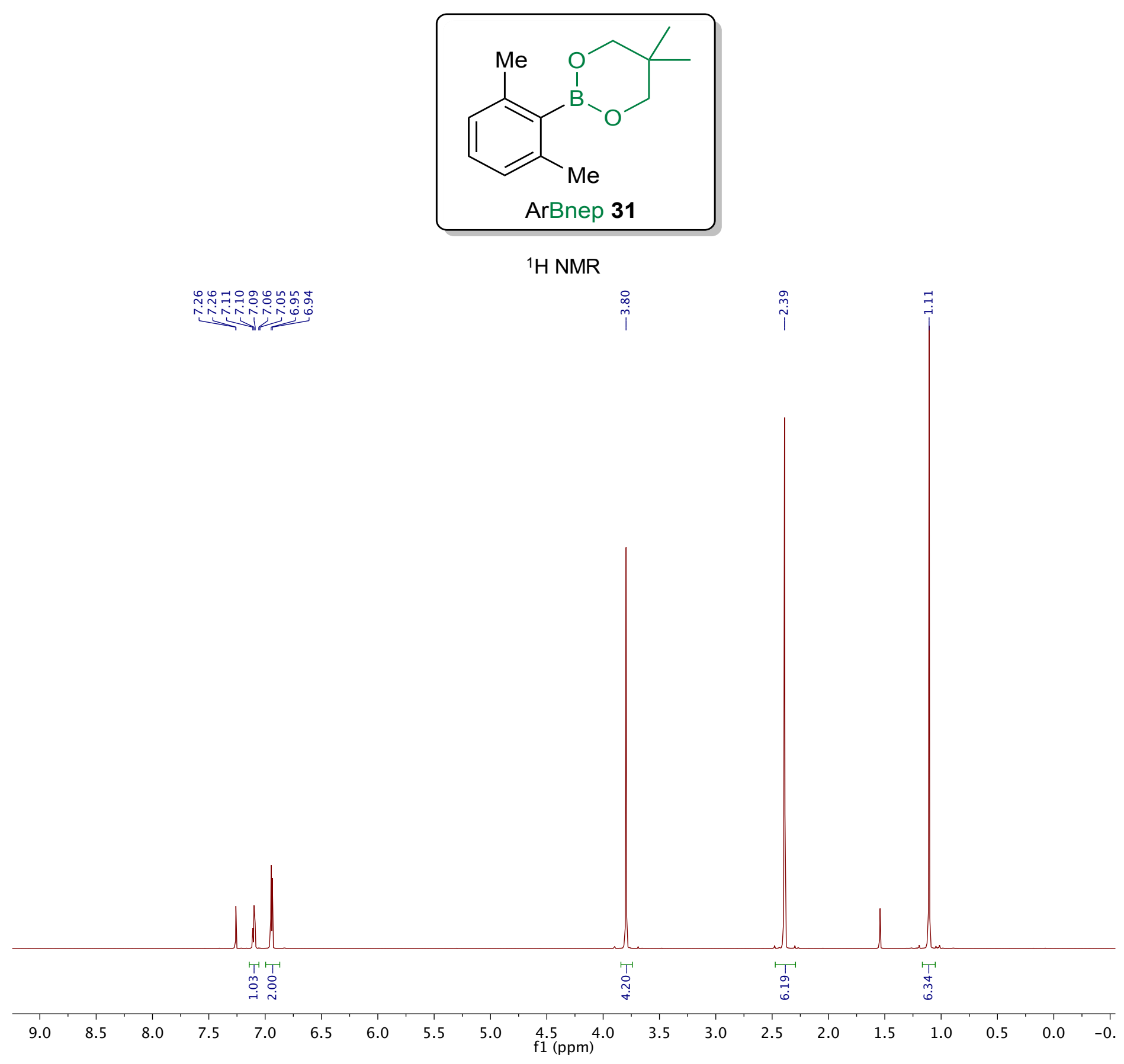




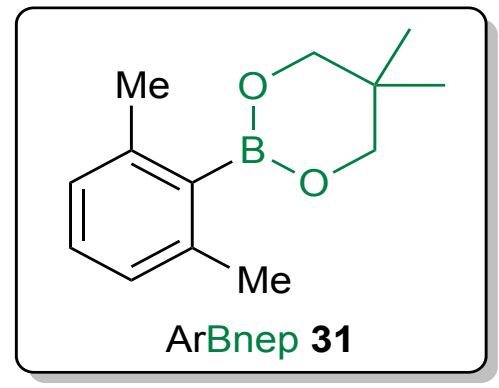

${ }^{13} \mathrm{C}$ NMR

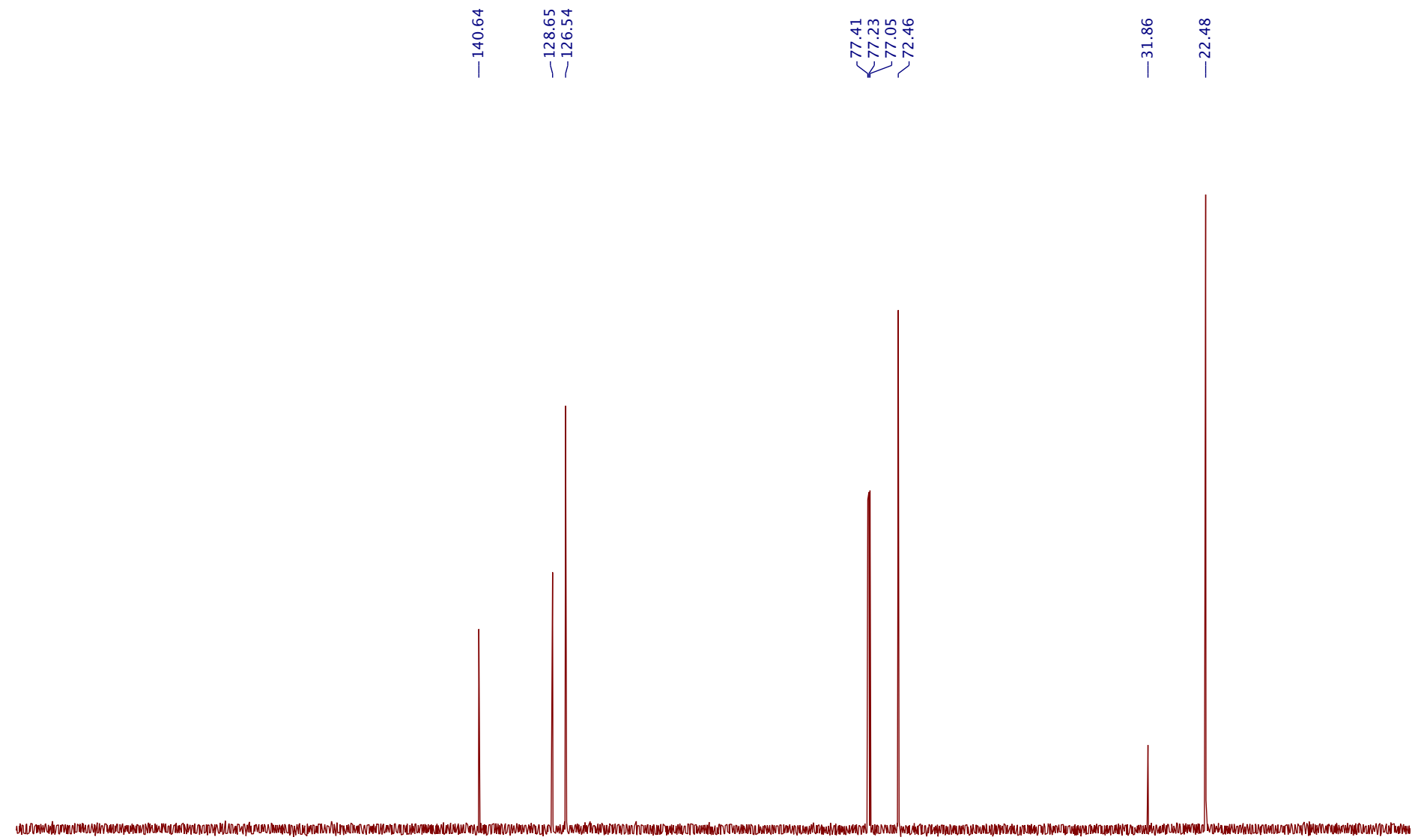

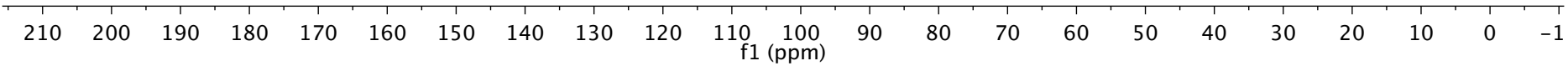




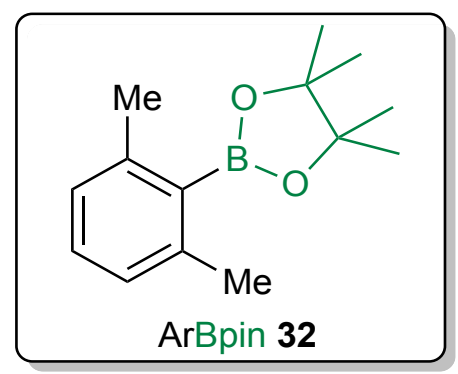

${ }^{1} \mathrm{H}$ NMR

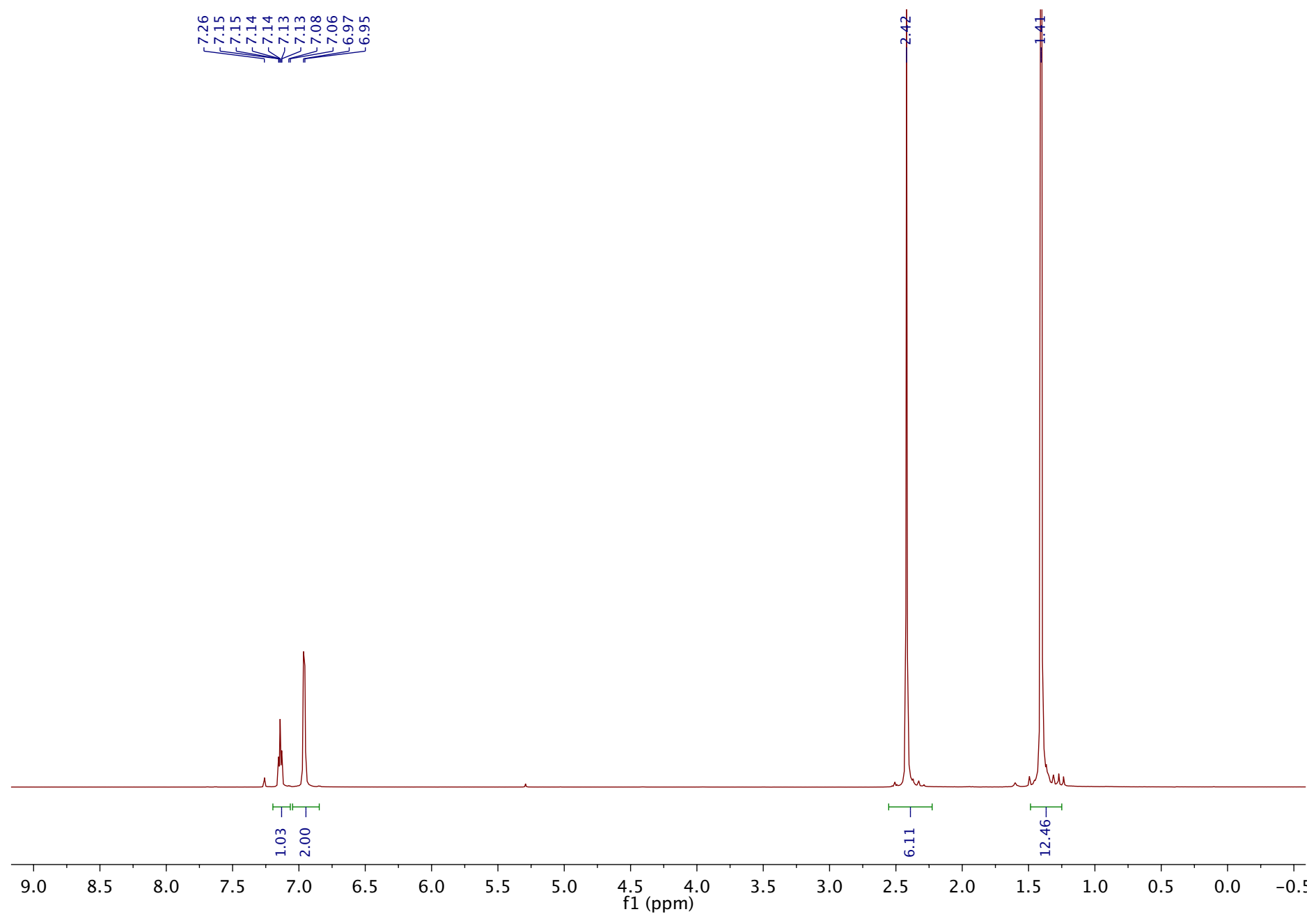




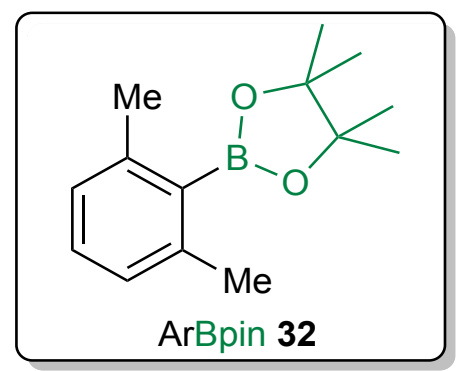

${ }^{13} \mathrm{C}$ NMR

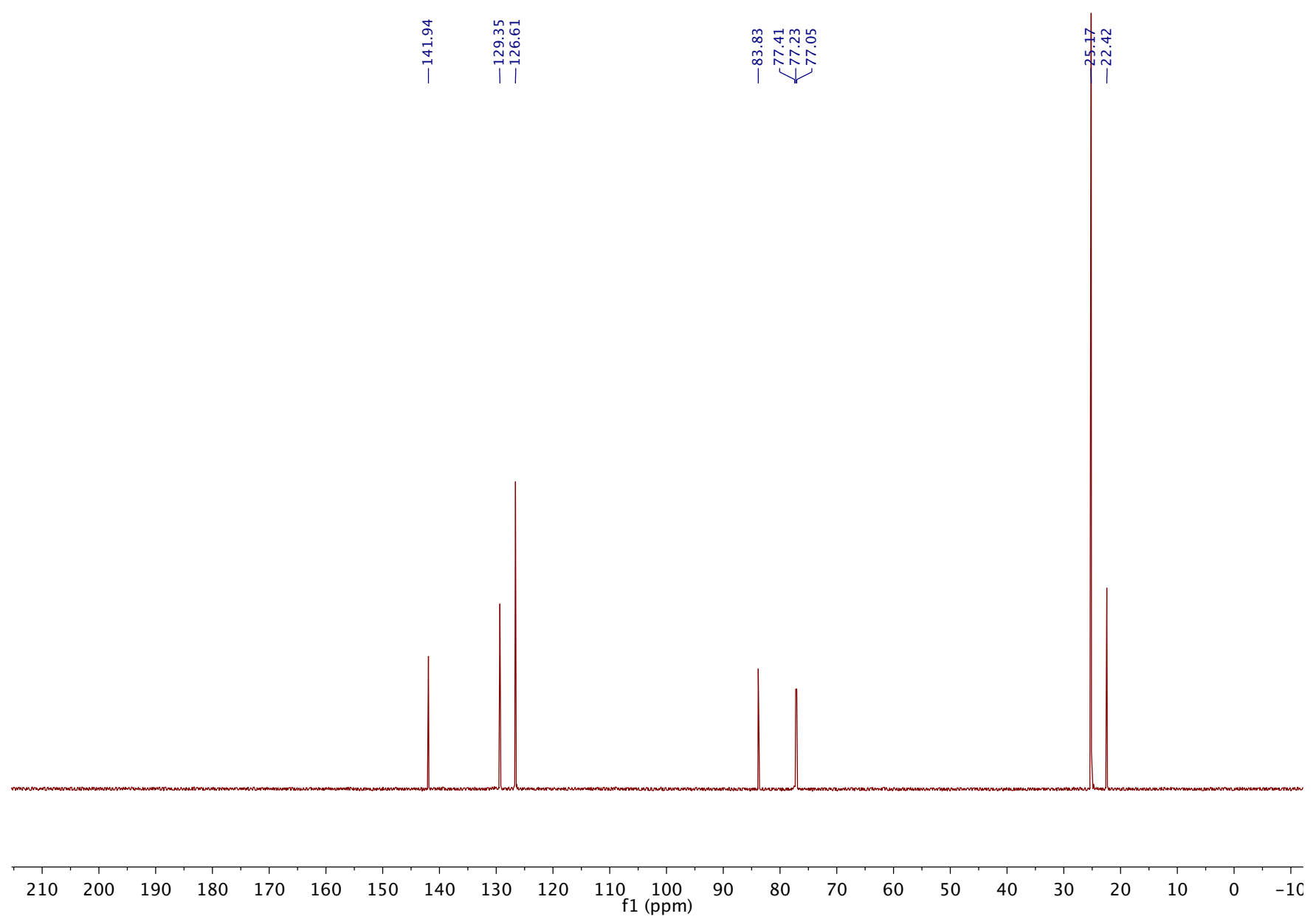




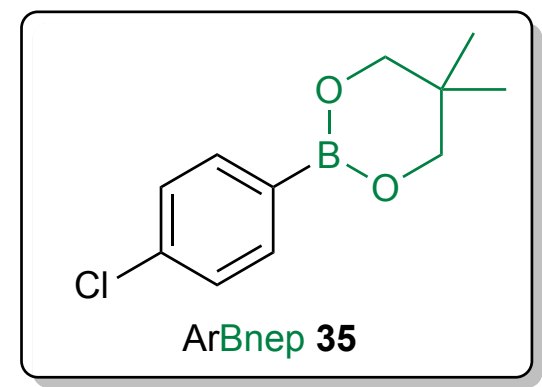

${ }^{1} \mathrm{H}$ NMR

제

तं त्र

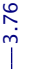

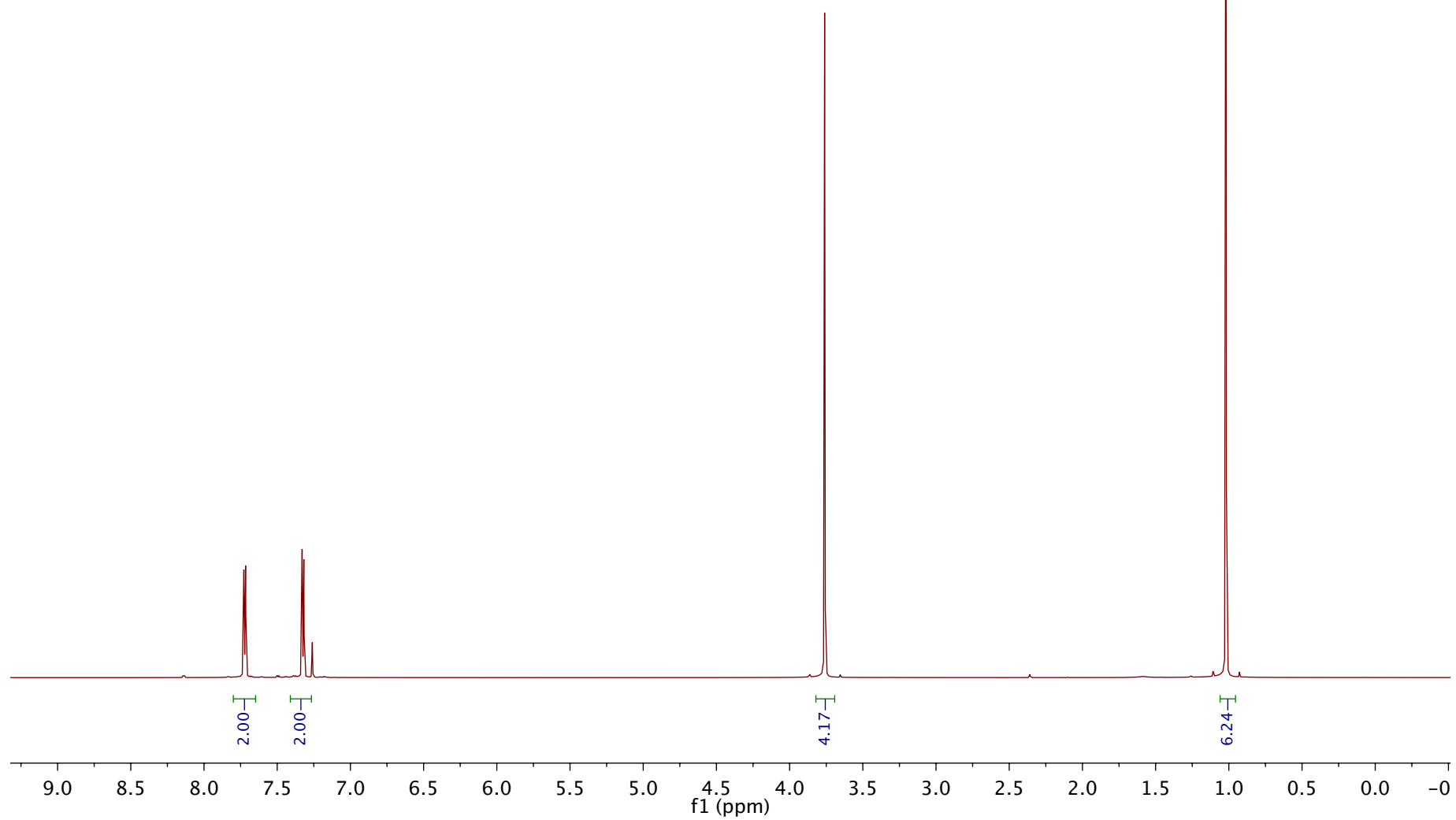




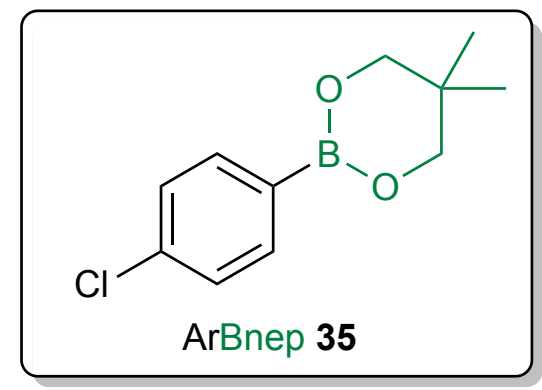

${ }^{13} \mathrm{C}$ NMR
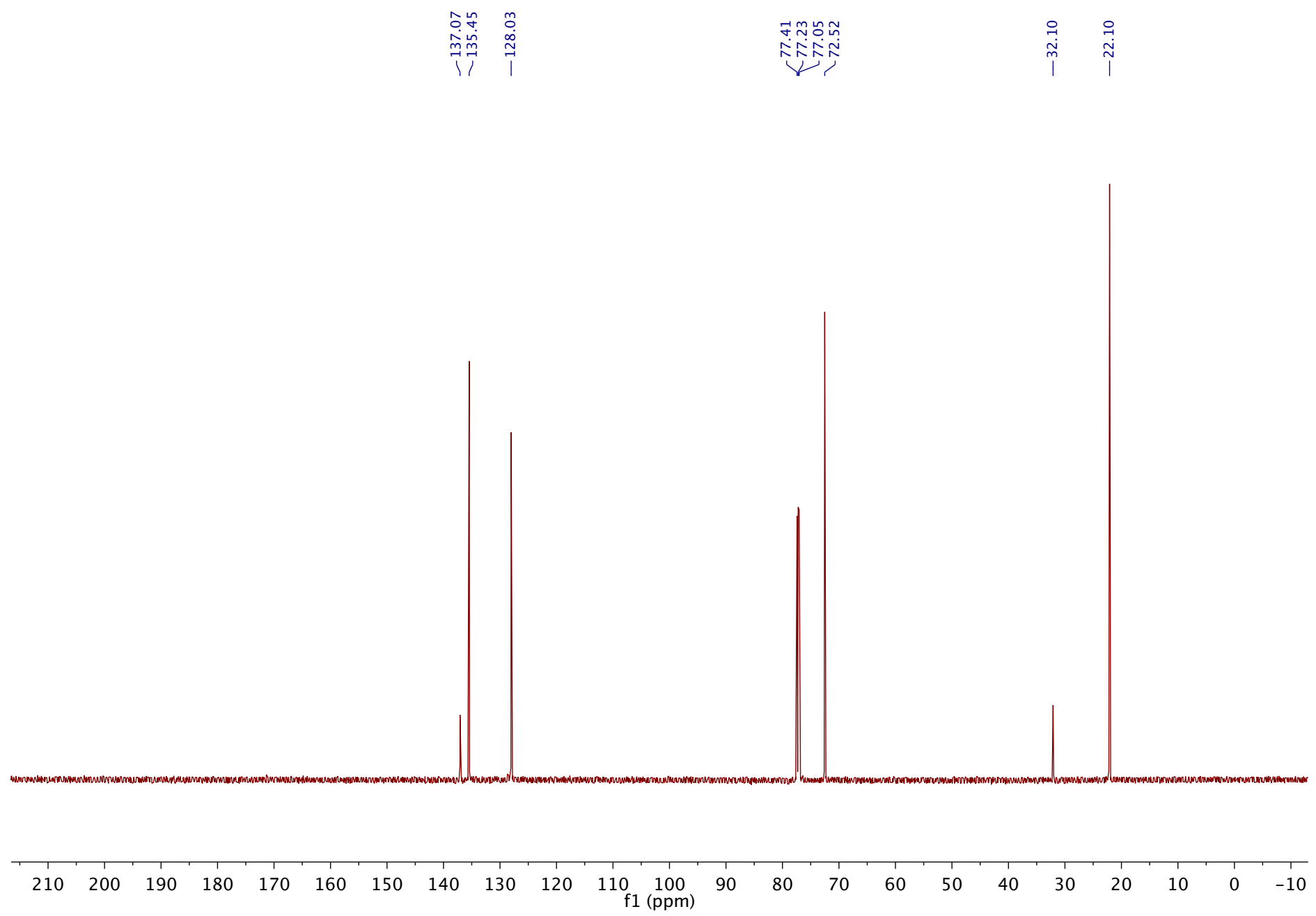


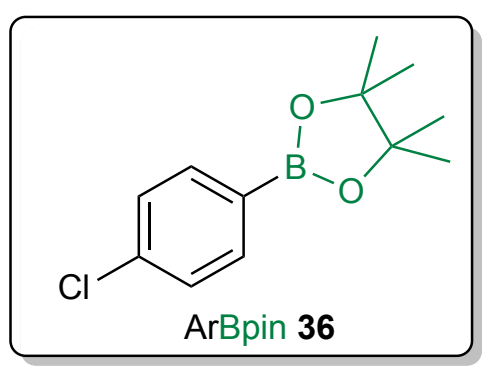

${ }^{1} \mathrm{H}$ NMR

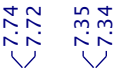

需

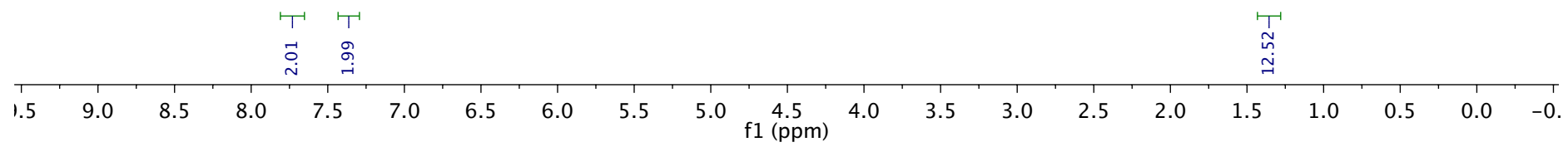




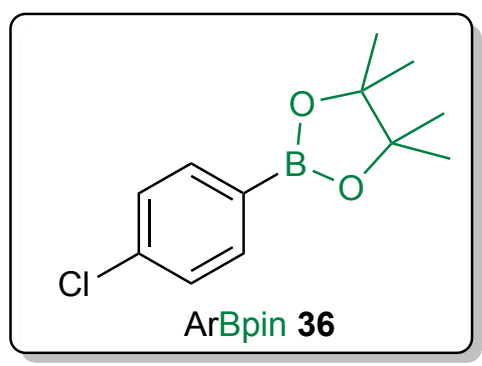

${ }^{13} \mathrm{C}$ NMR
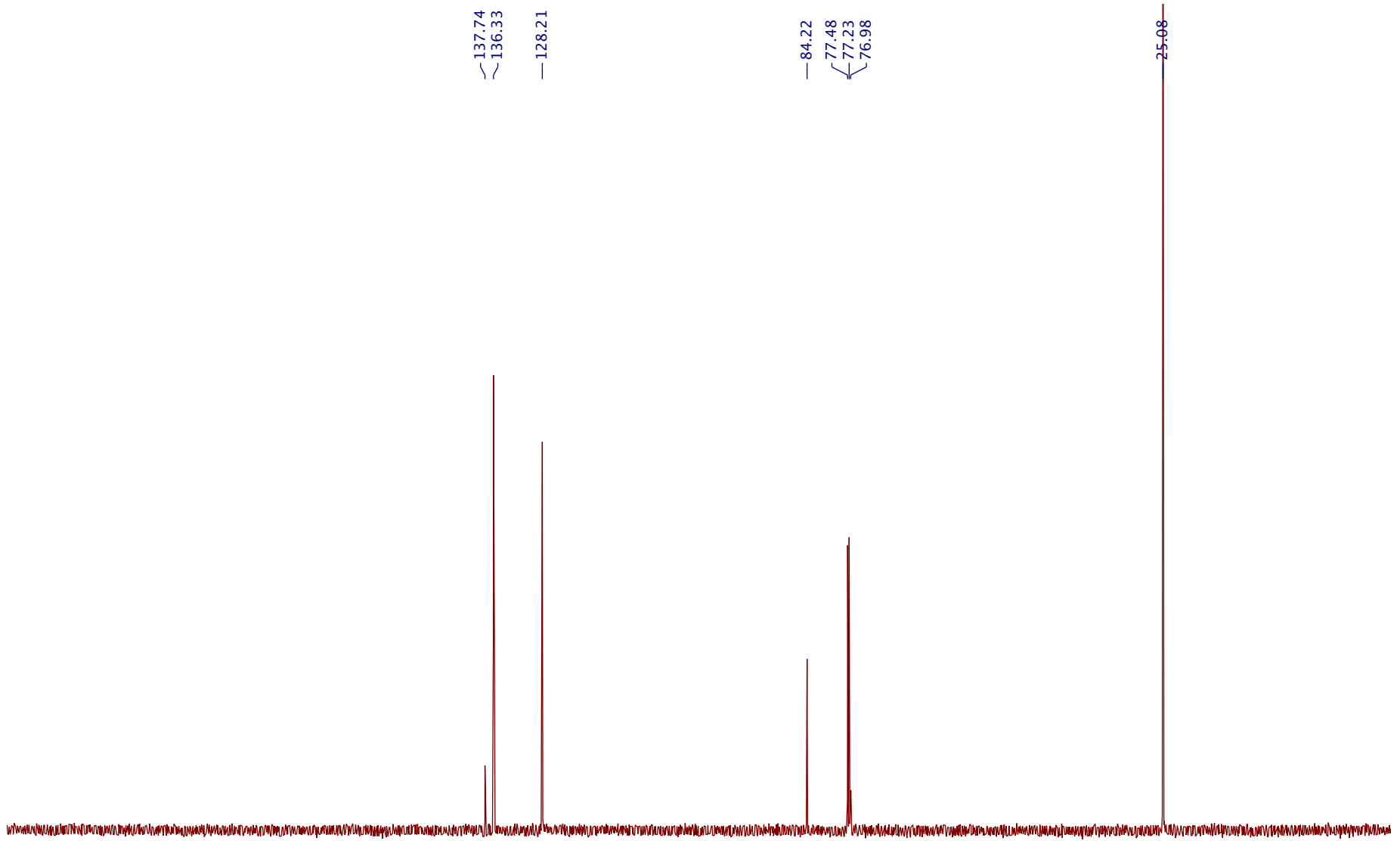

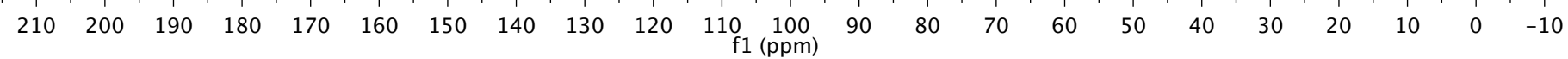




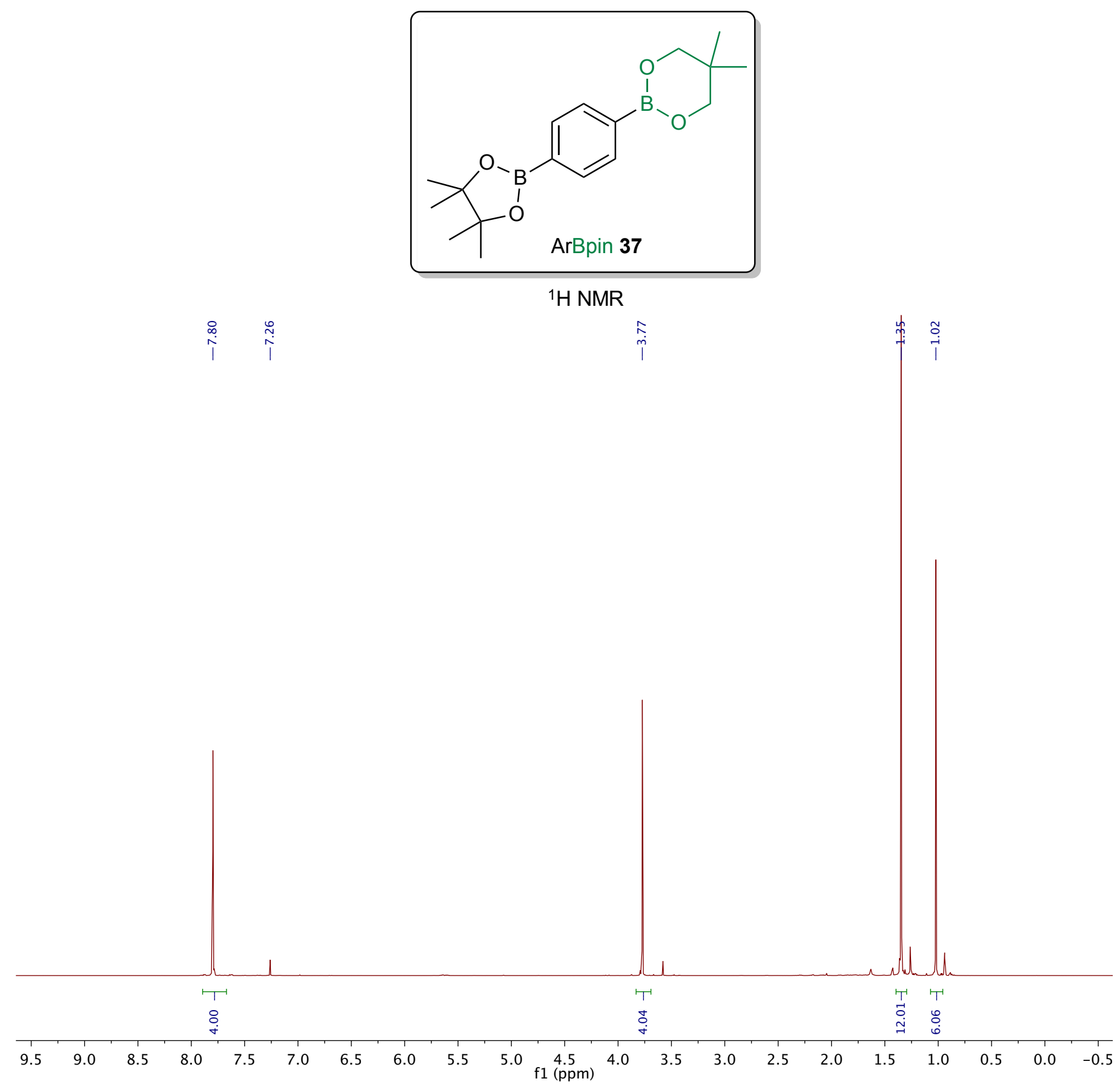



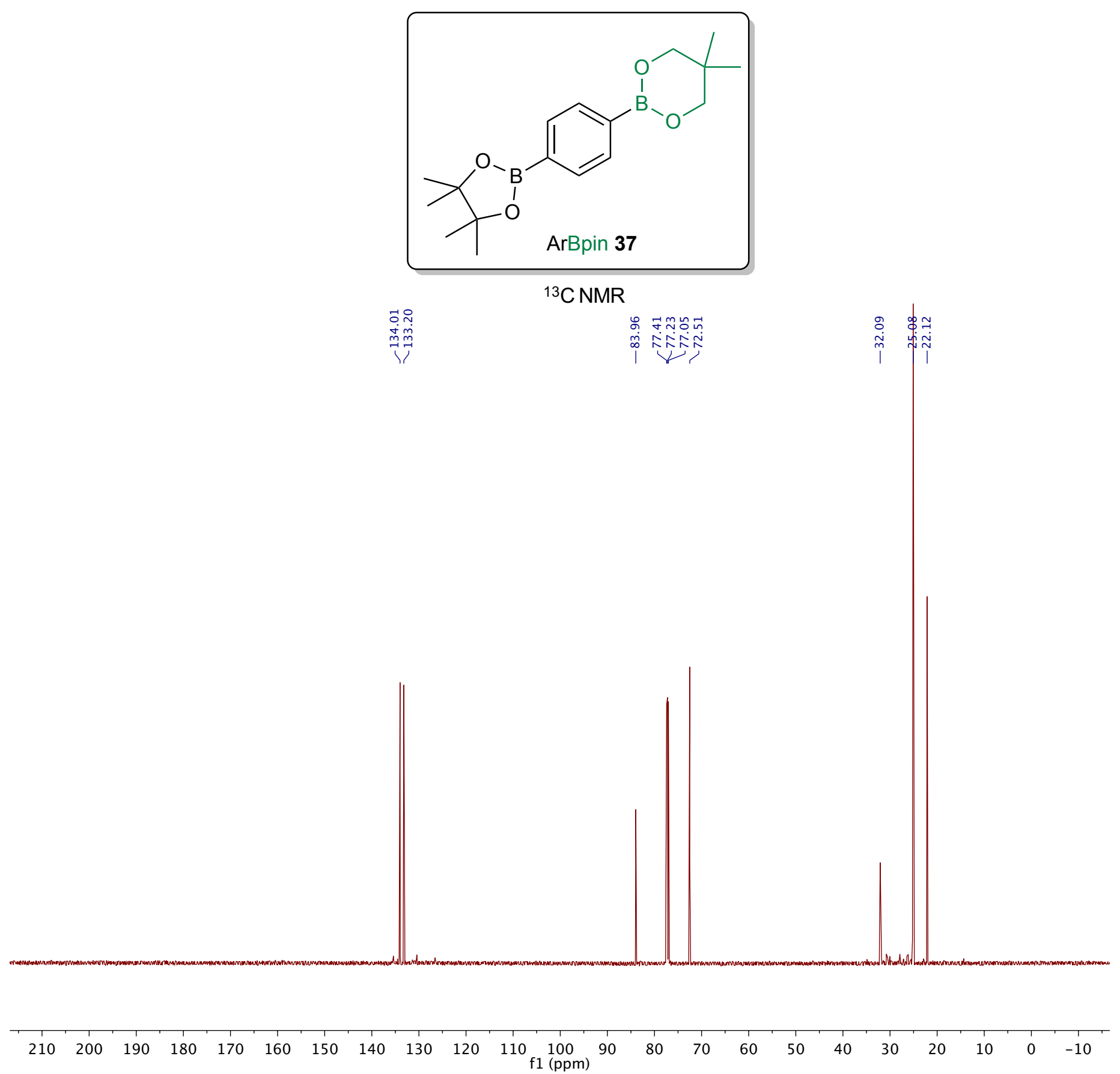


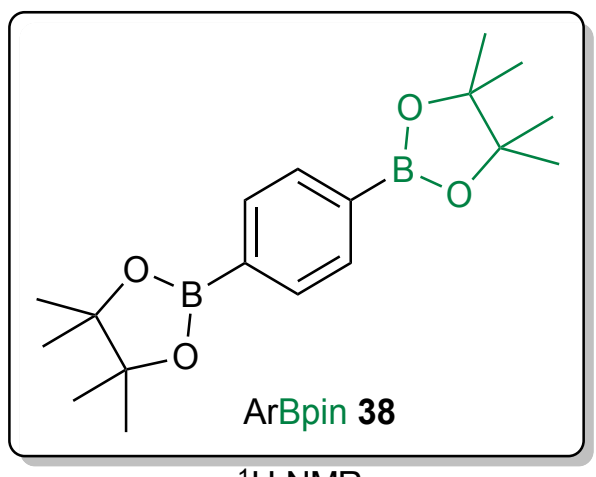

${ }^{1} \mathrm{H}$ NMR

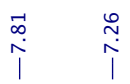

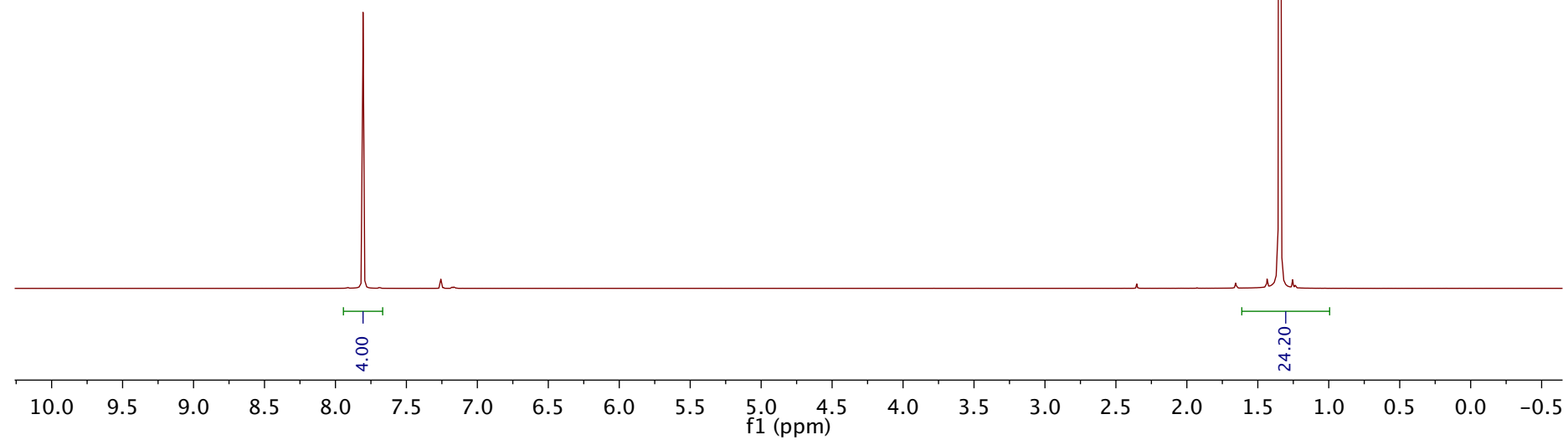




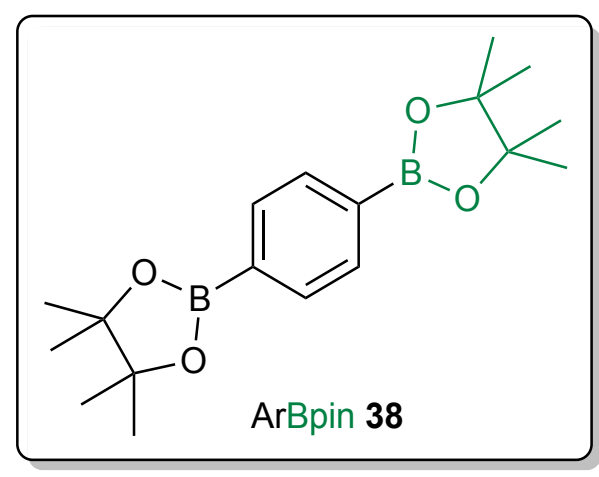

${ }^{13} \mathrm{C}$ NMR
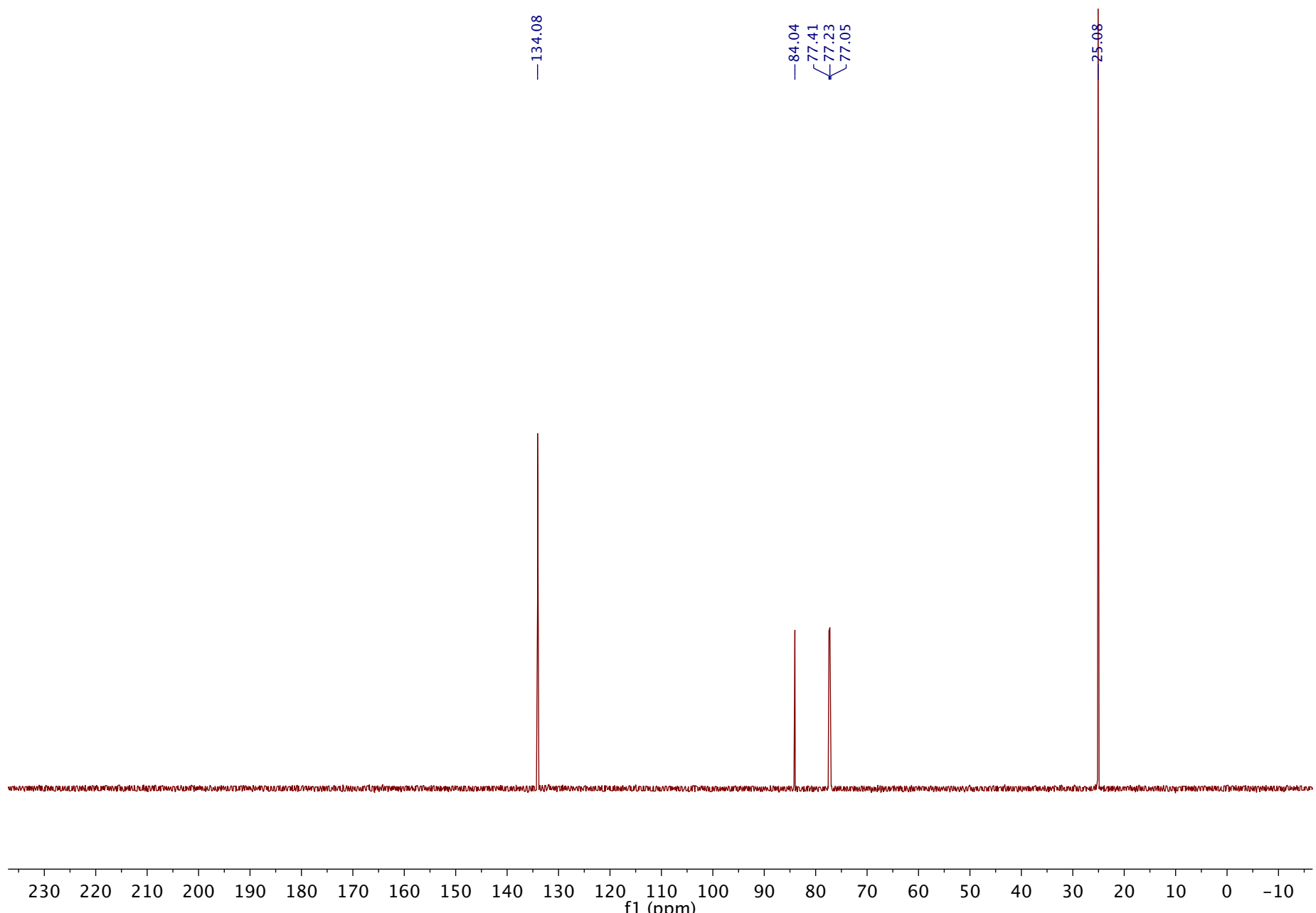


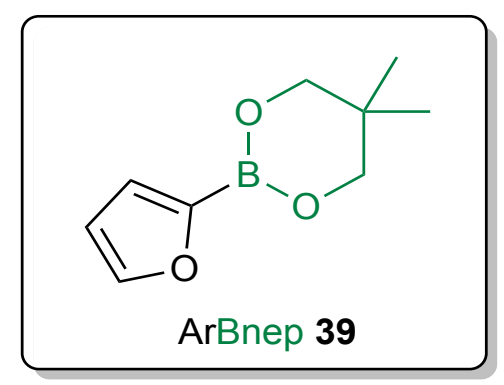

${ }^{1} \mathrm{H}$ NMR

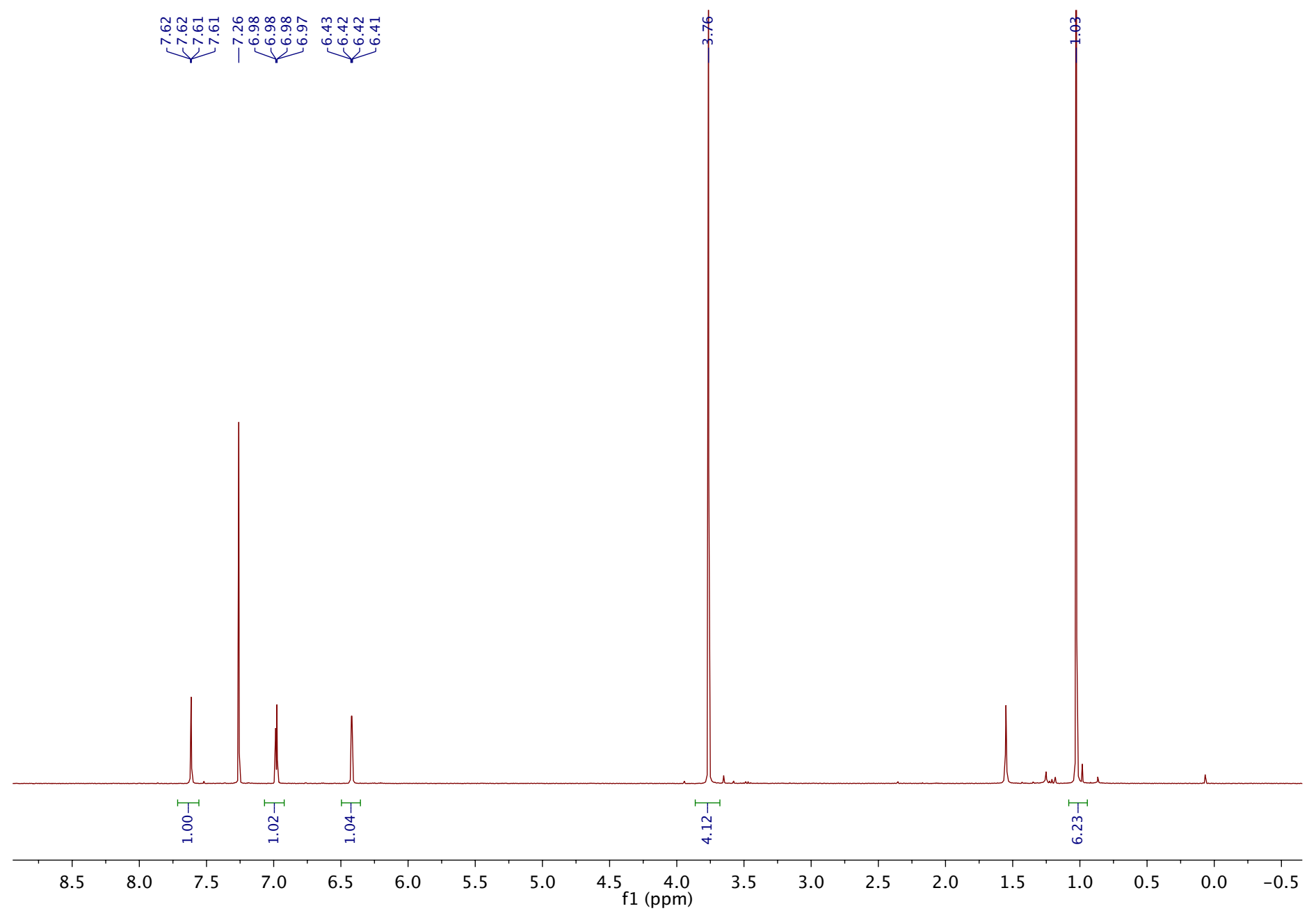




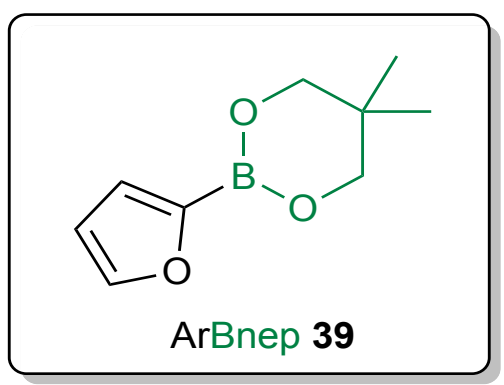

${ }^{13} \mathrm{C}$ NMR

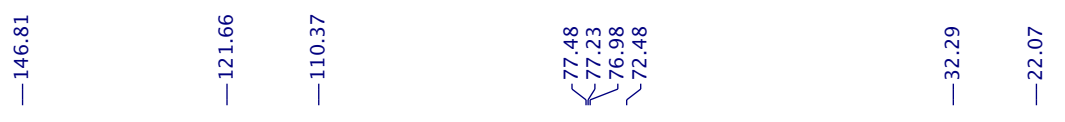

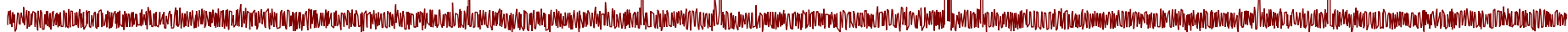

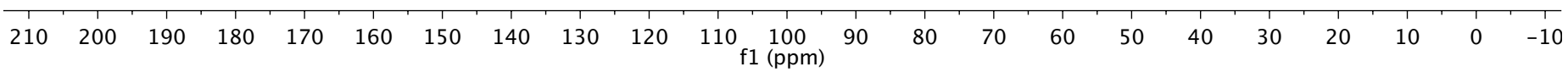




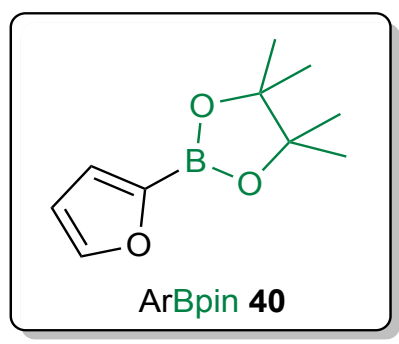

${ }^{1} \mathrm{H}$ NMR
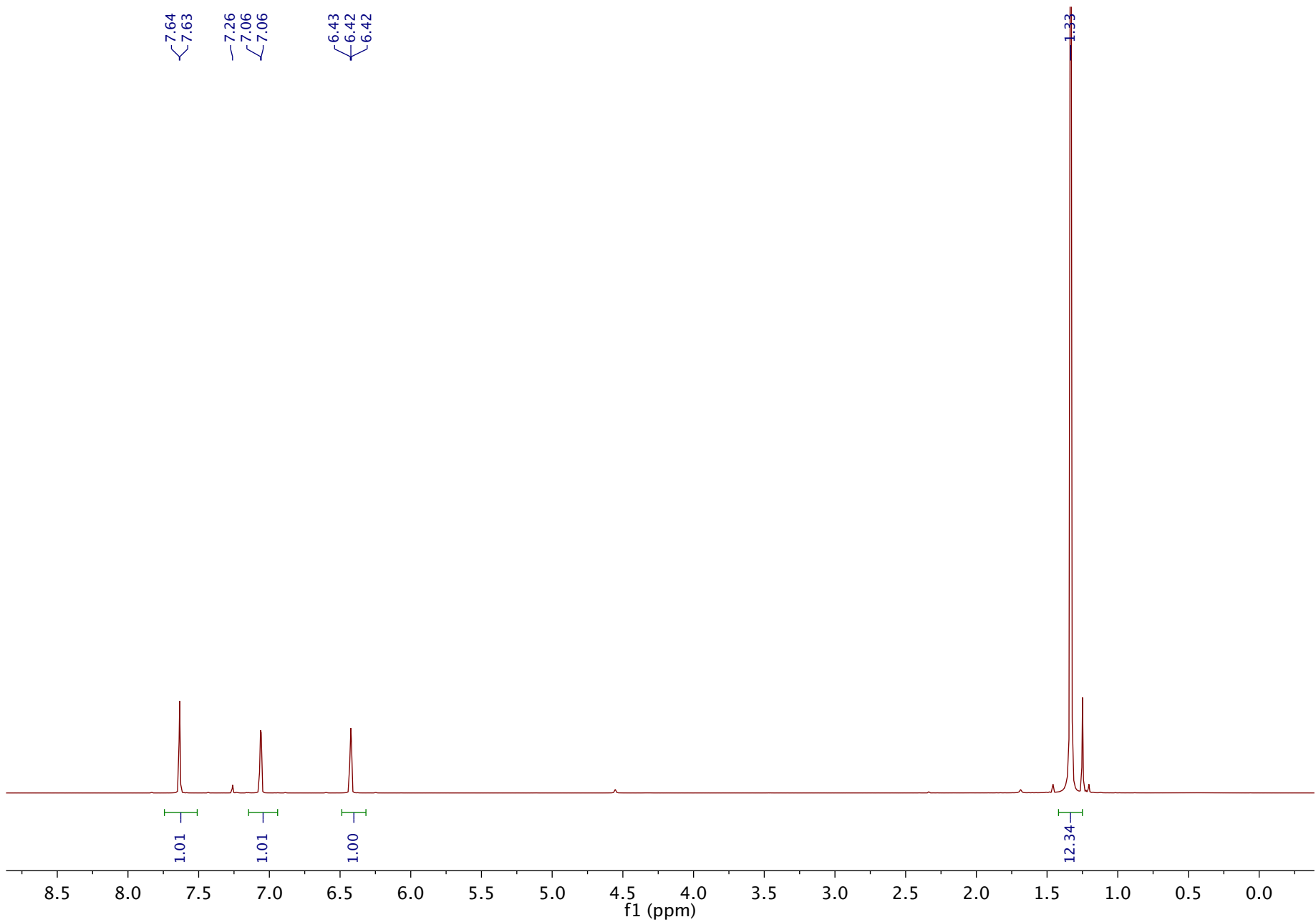

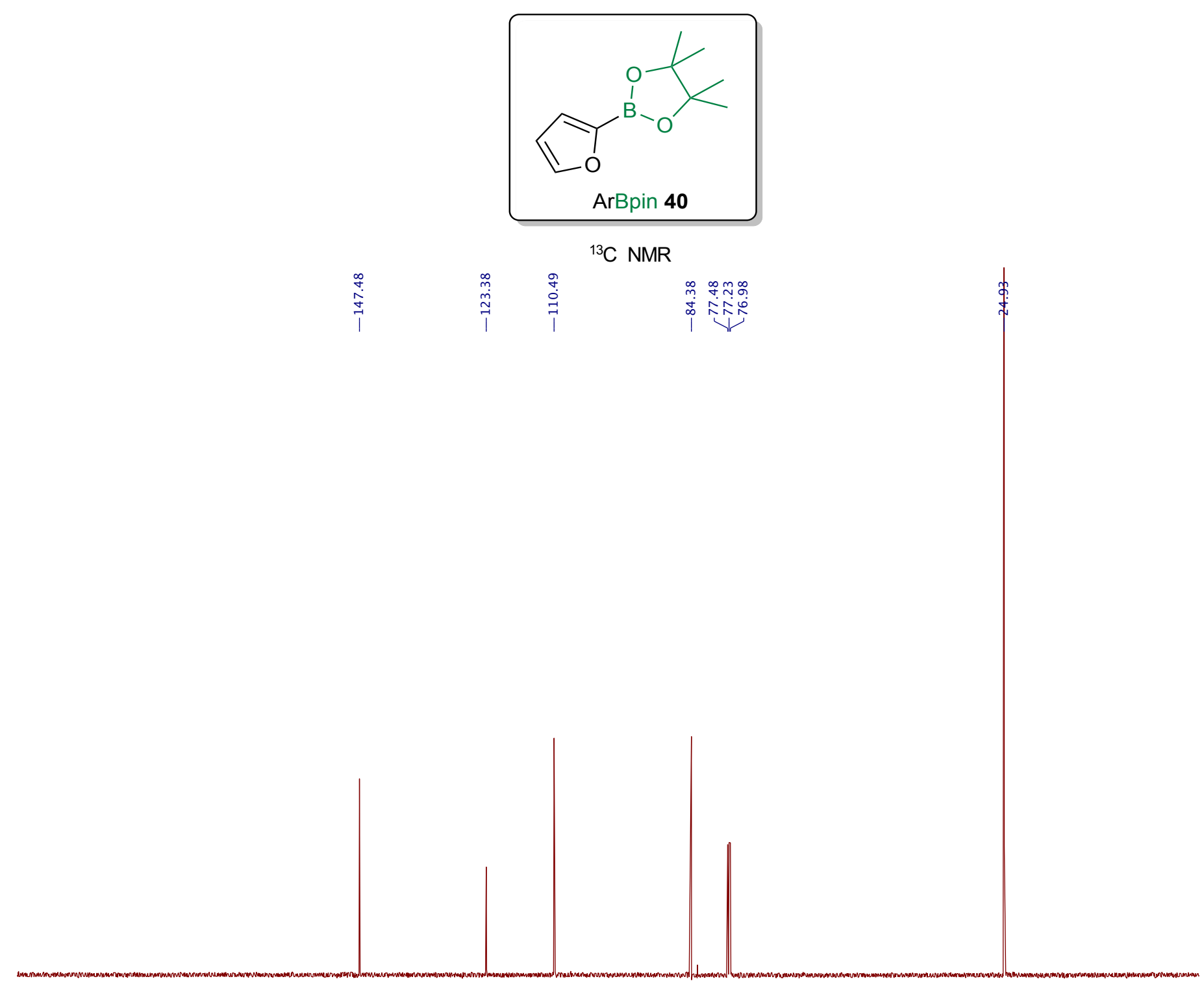

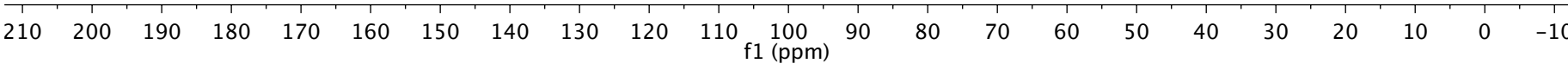




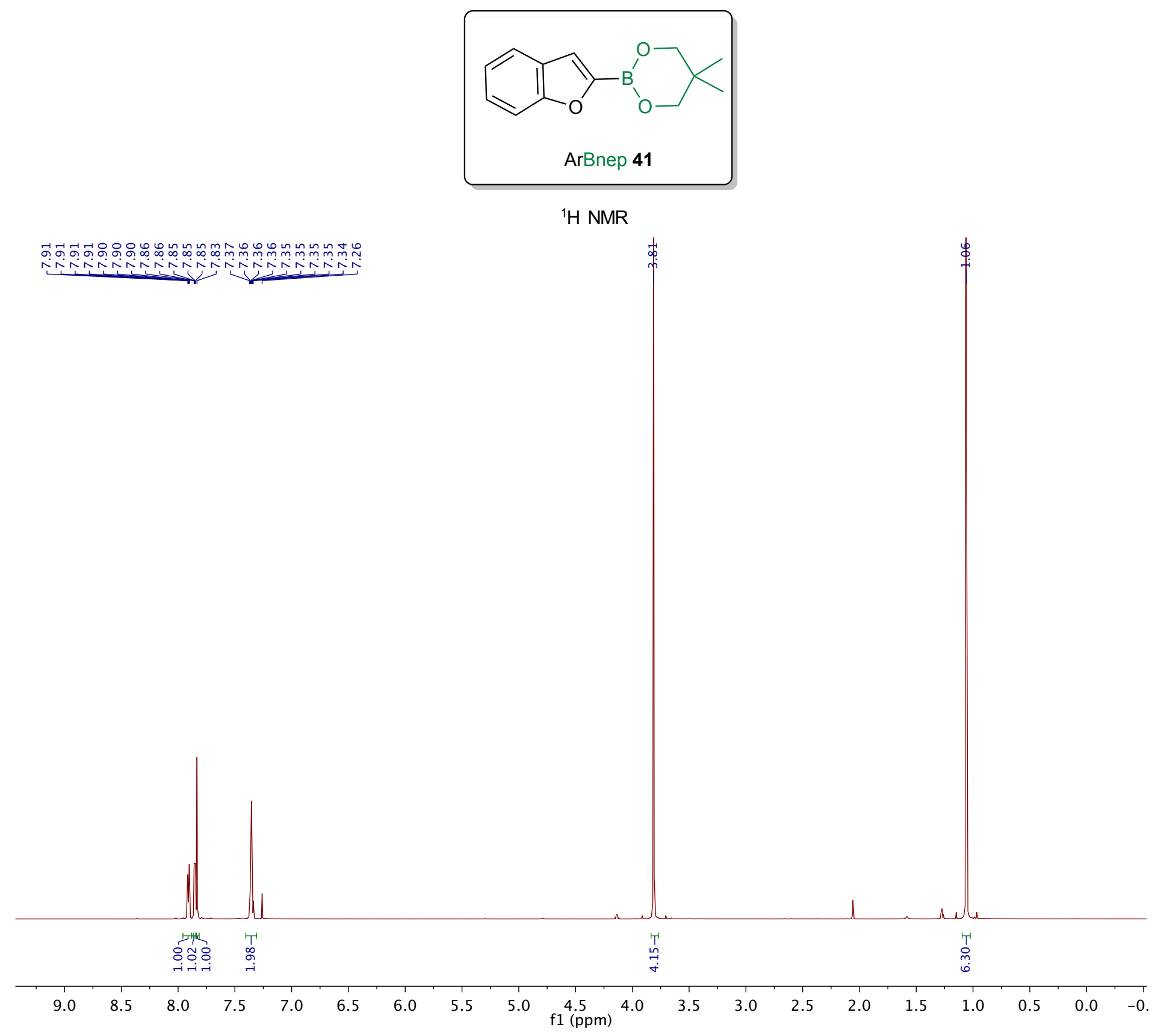




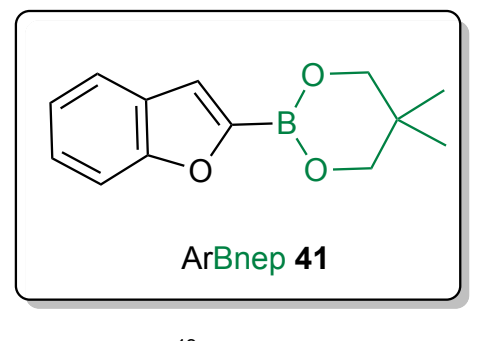

${ }^{13} \mathrm{C}$ NMR

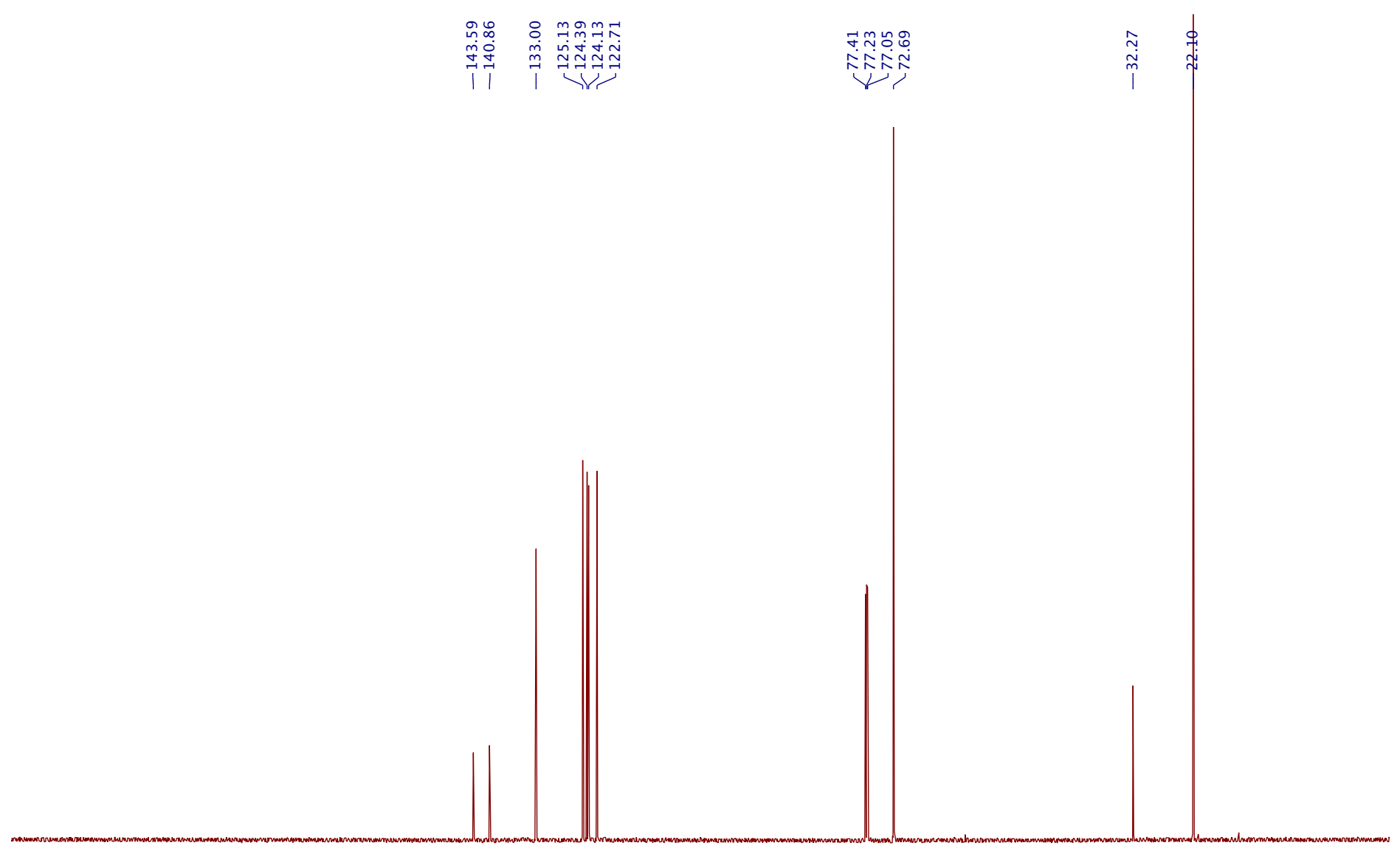

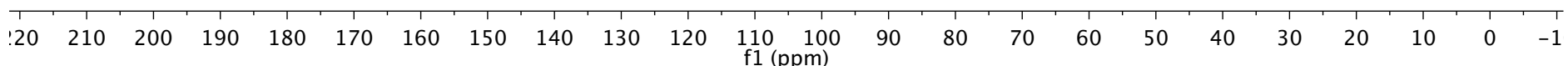




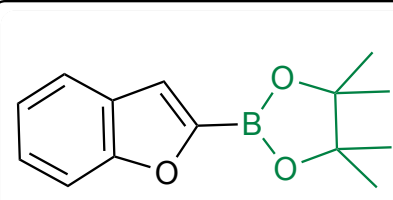

ArBpin 42

${ }^{1} \mathrm{H}$ NMR

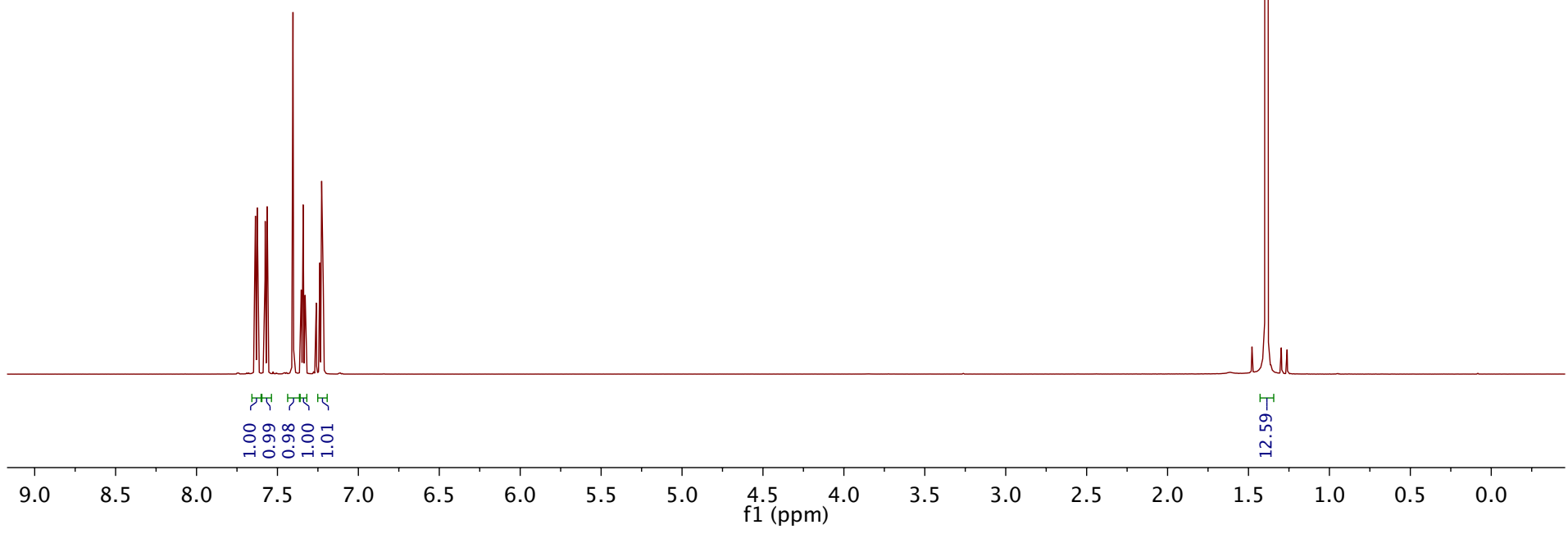



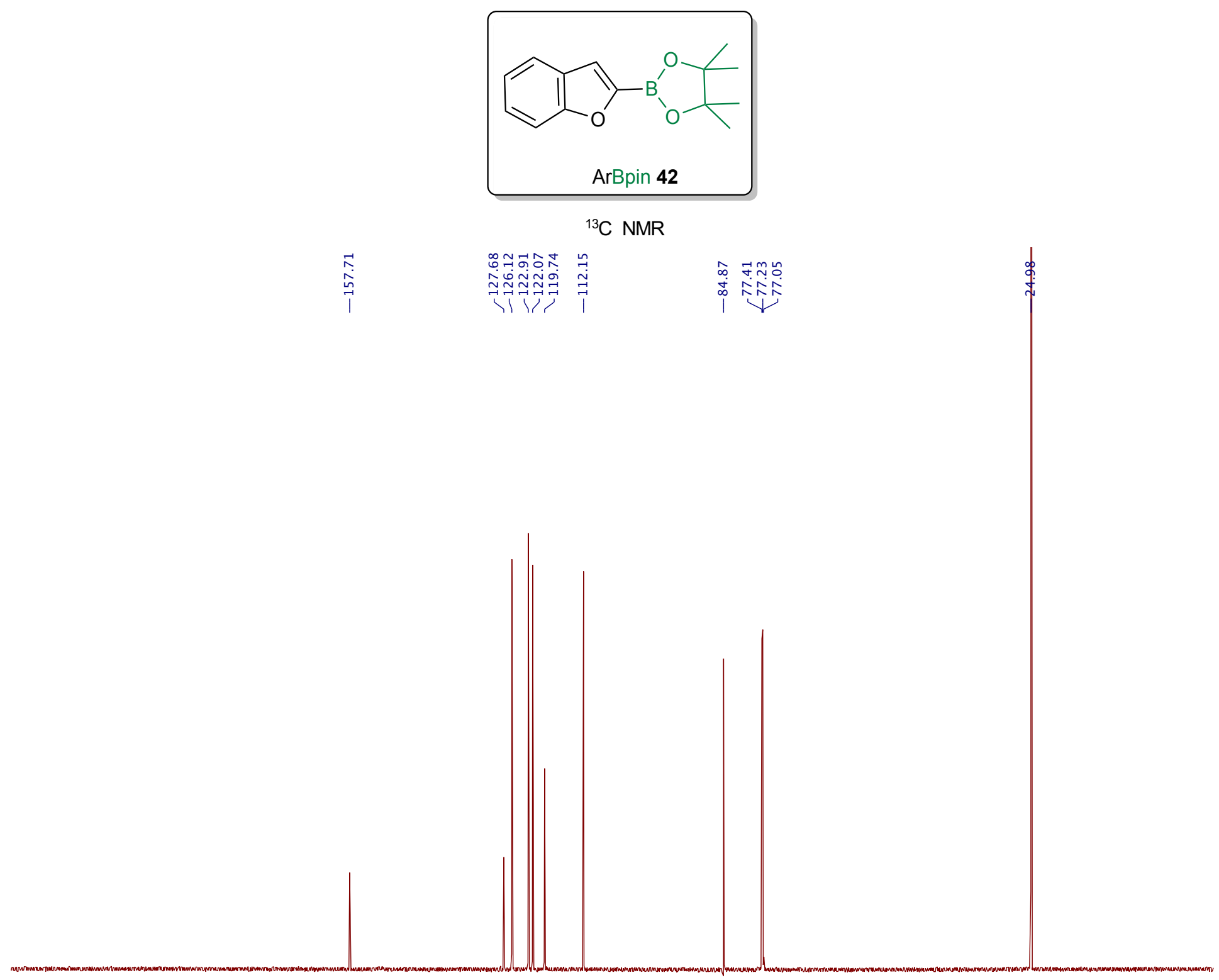


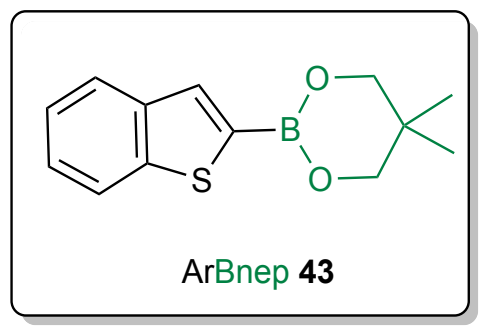

${ }^{1} \mathrm{H}$ NMR

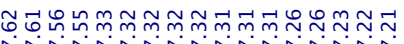

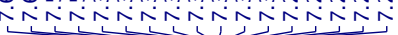

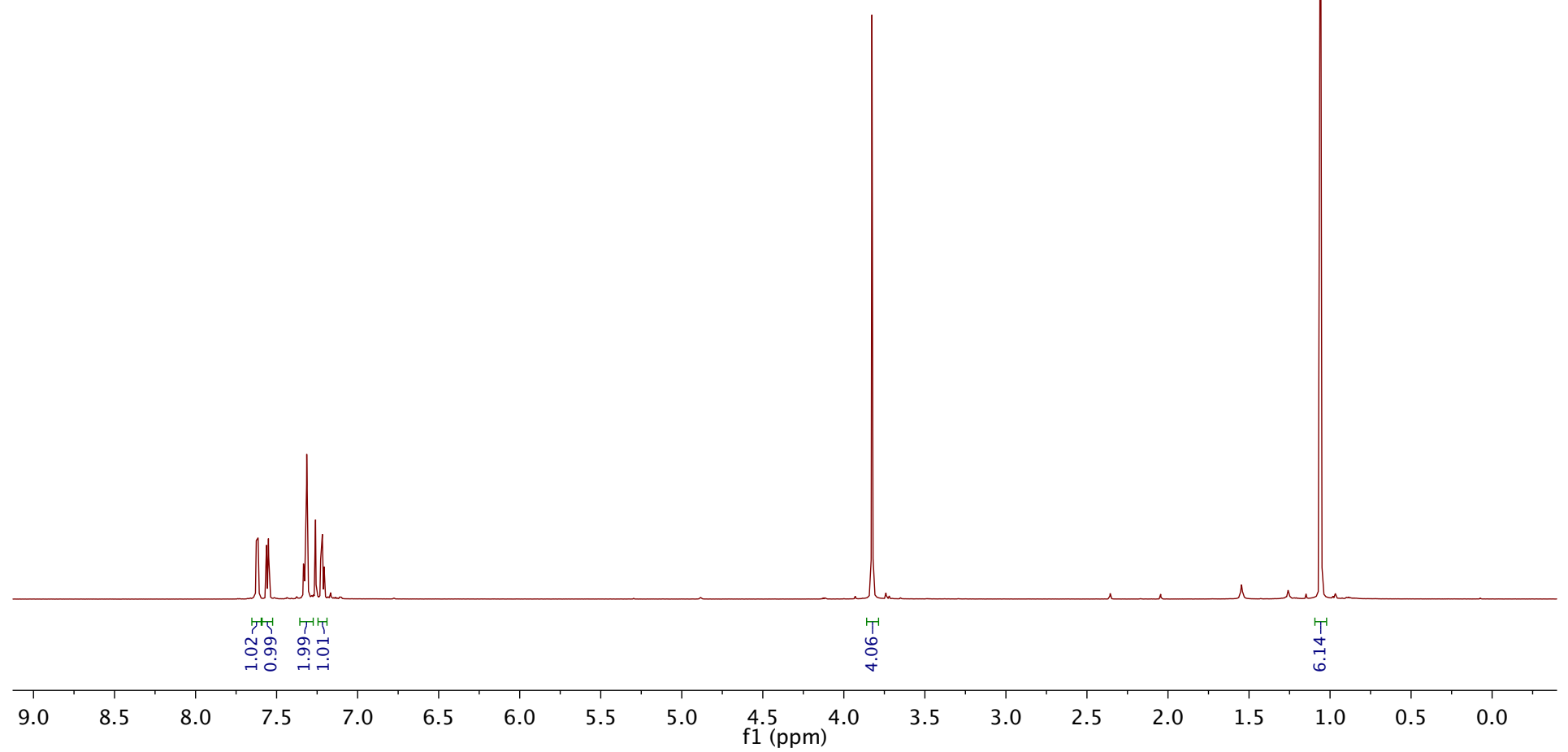




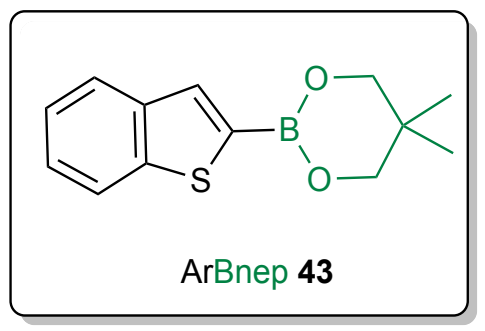

${ }^{13} \mathrm{C}$ NMR

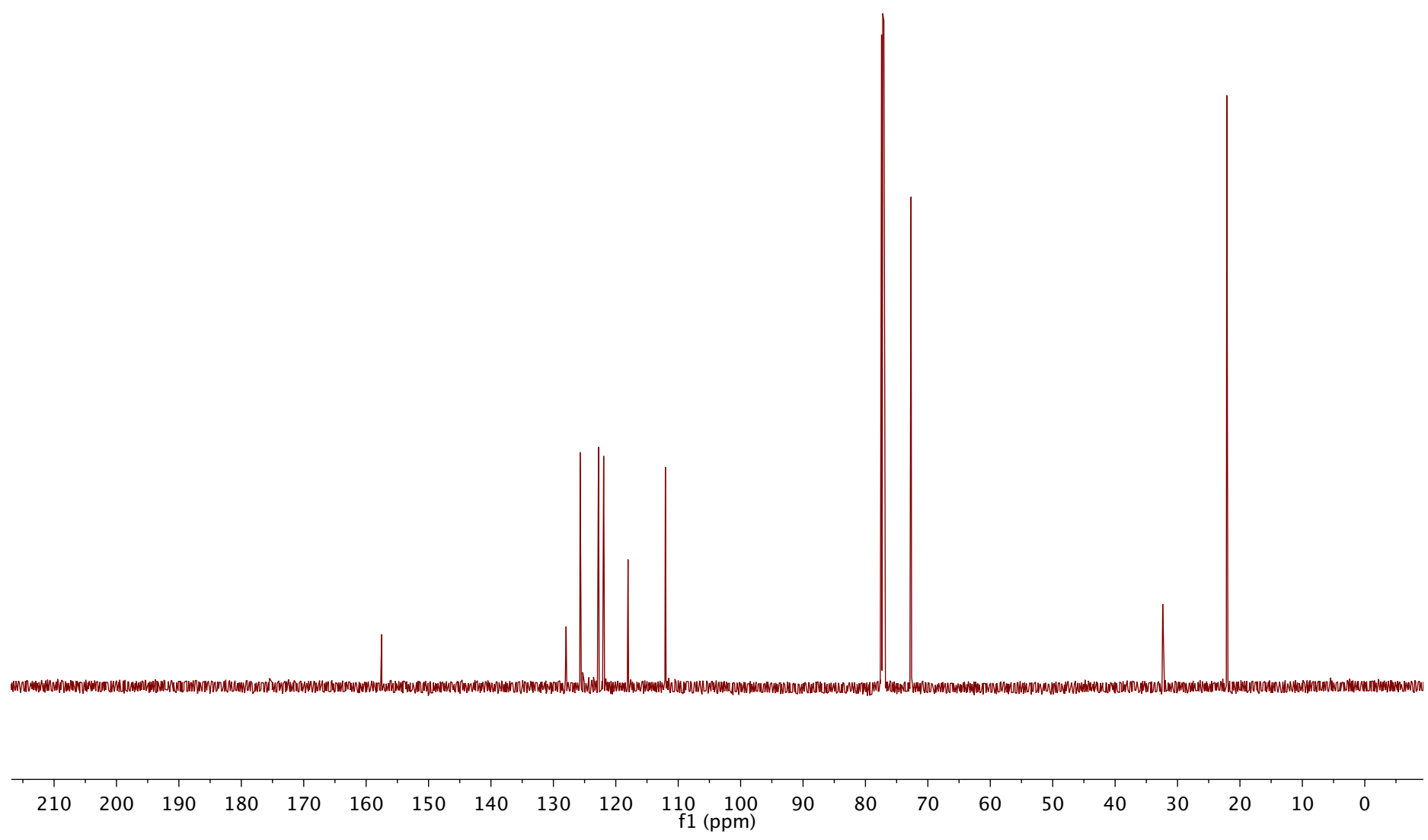




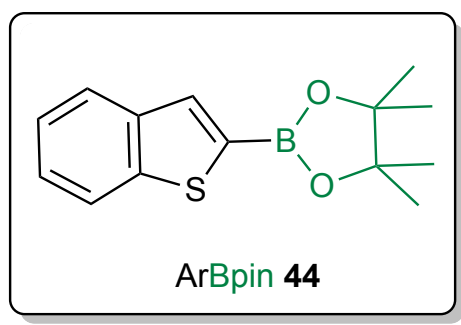

${ }^{1} \mathrm{H}$ NMR

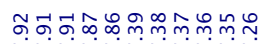

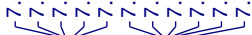

(

$\oiint_{+}$

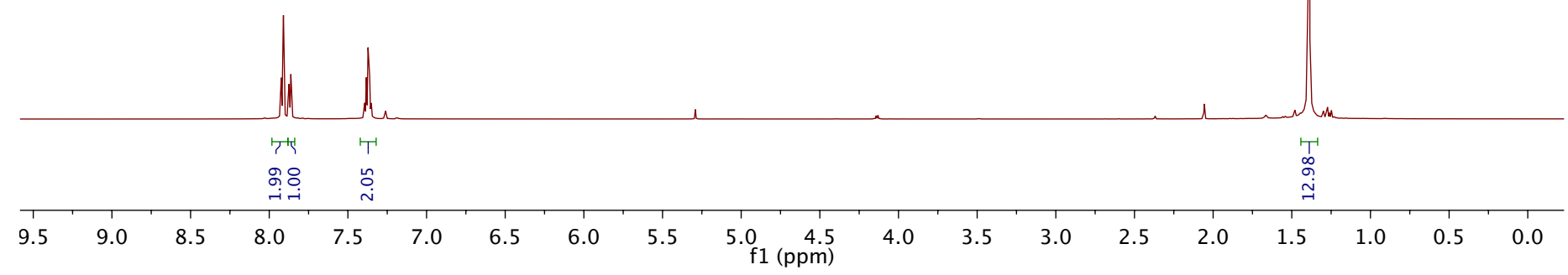




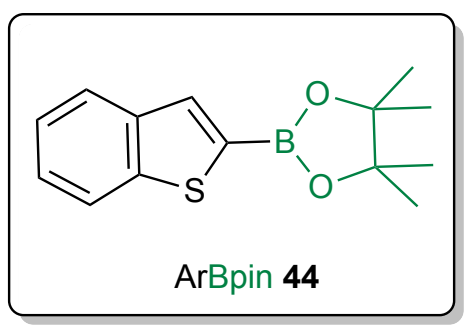

${ }^{13} \mathrm{C}$ NMR
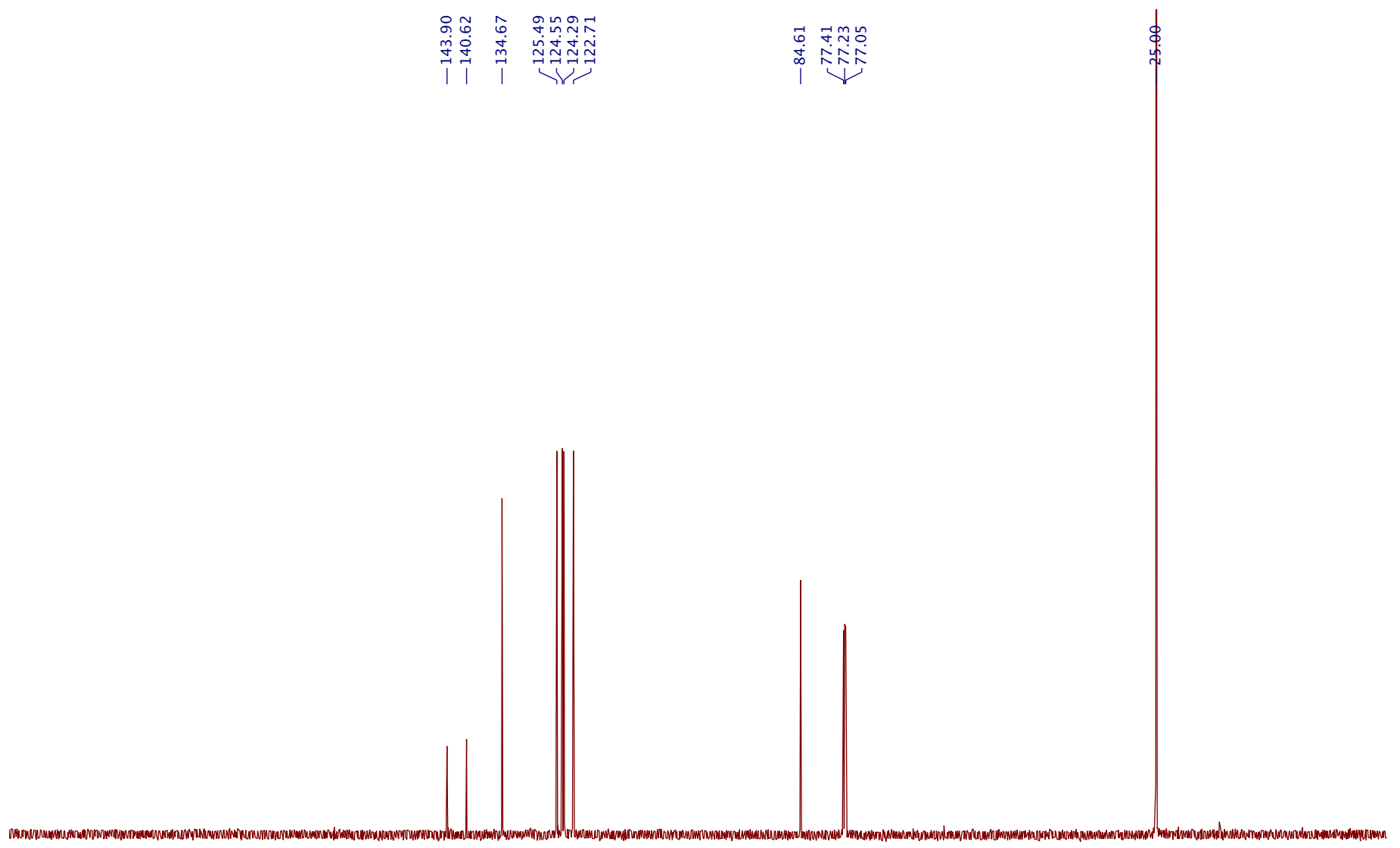

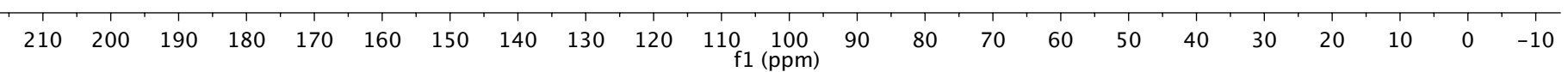




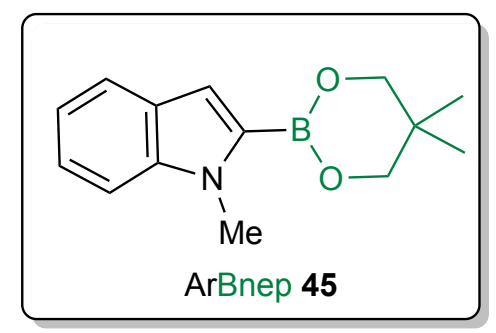

${ }^{1} \mathrm{H}$ NMR

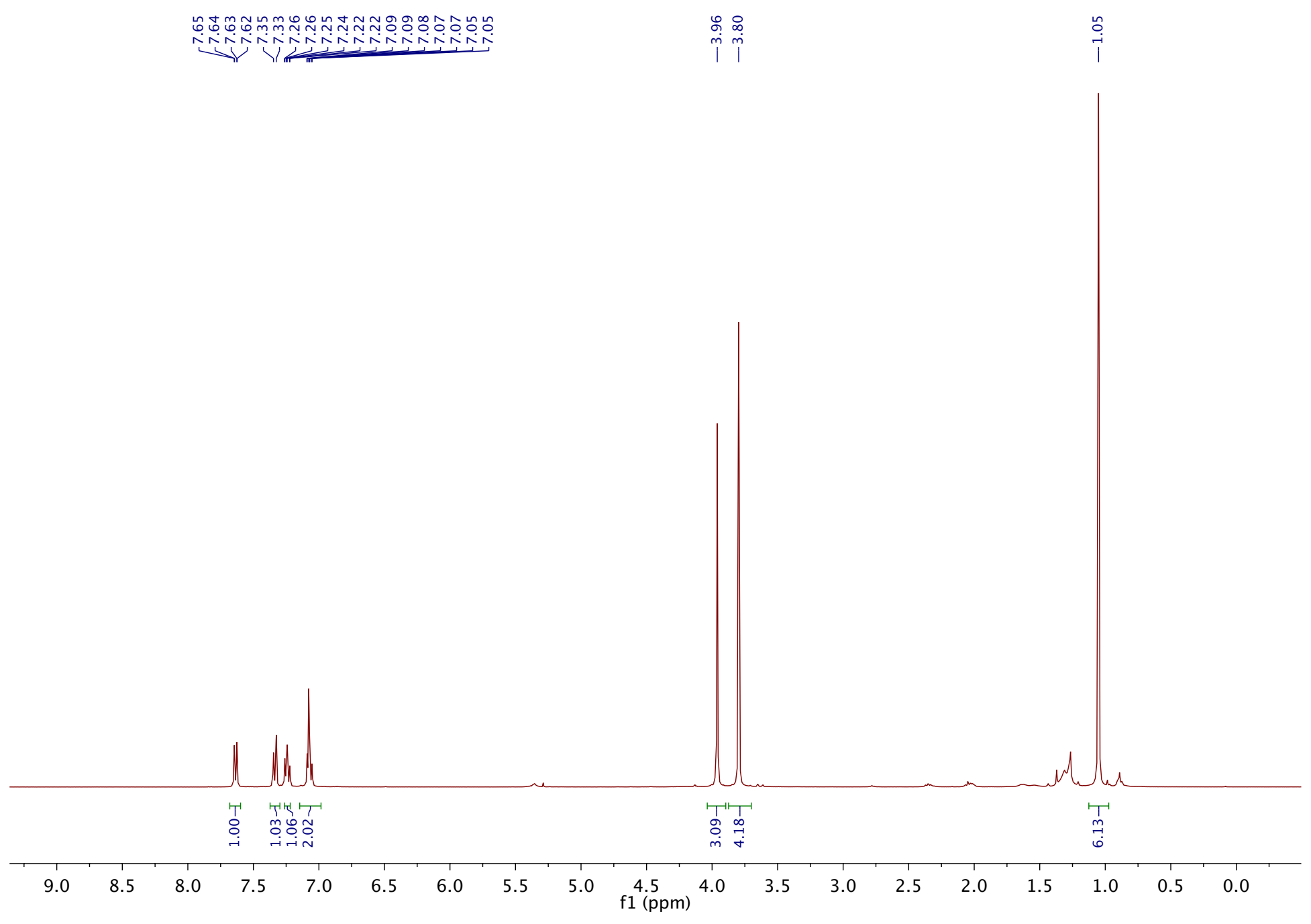



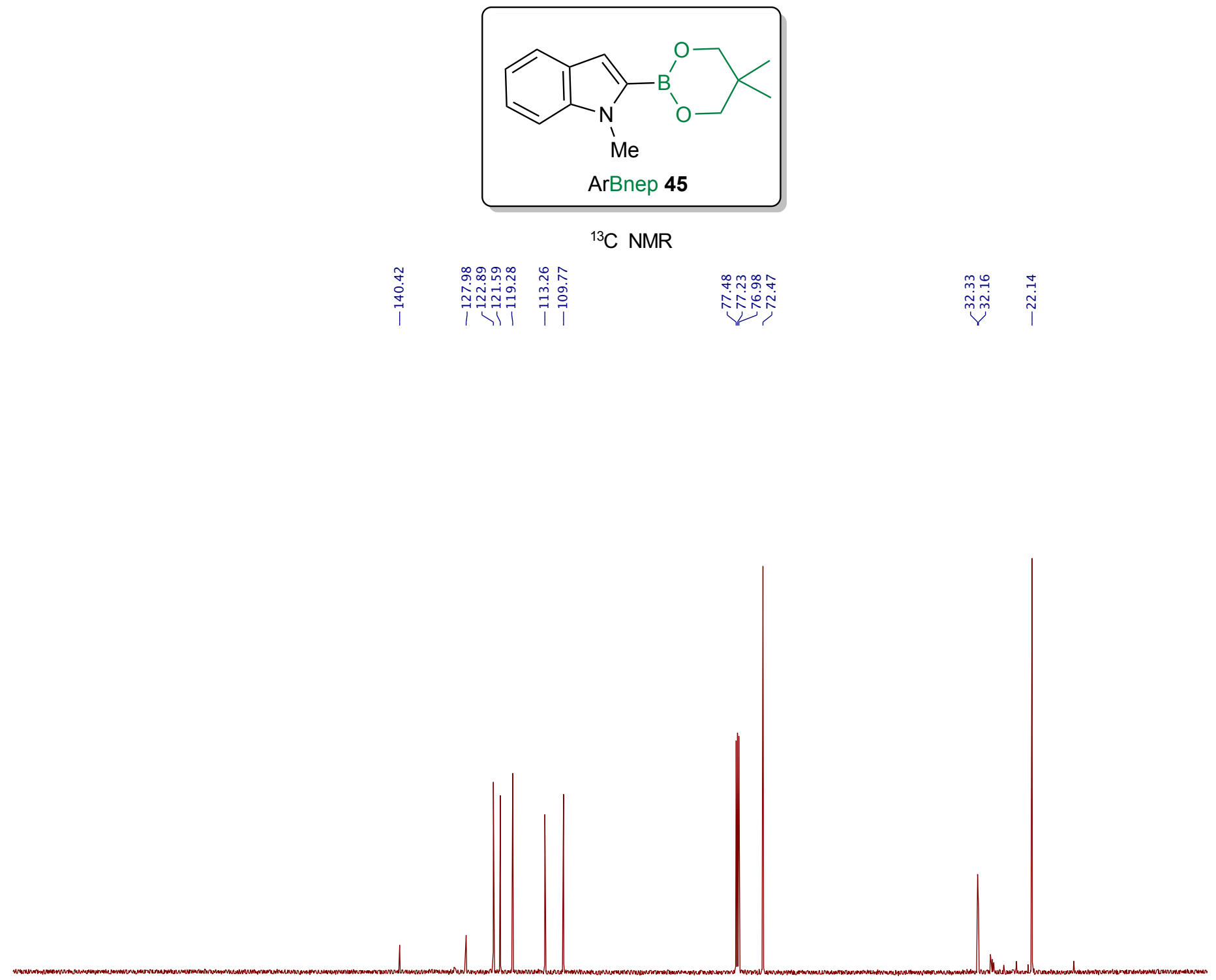

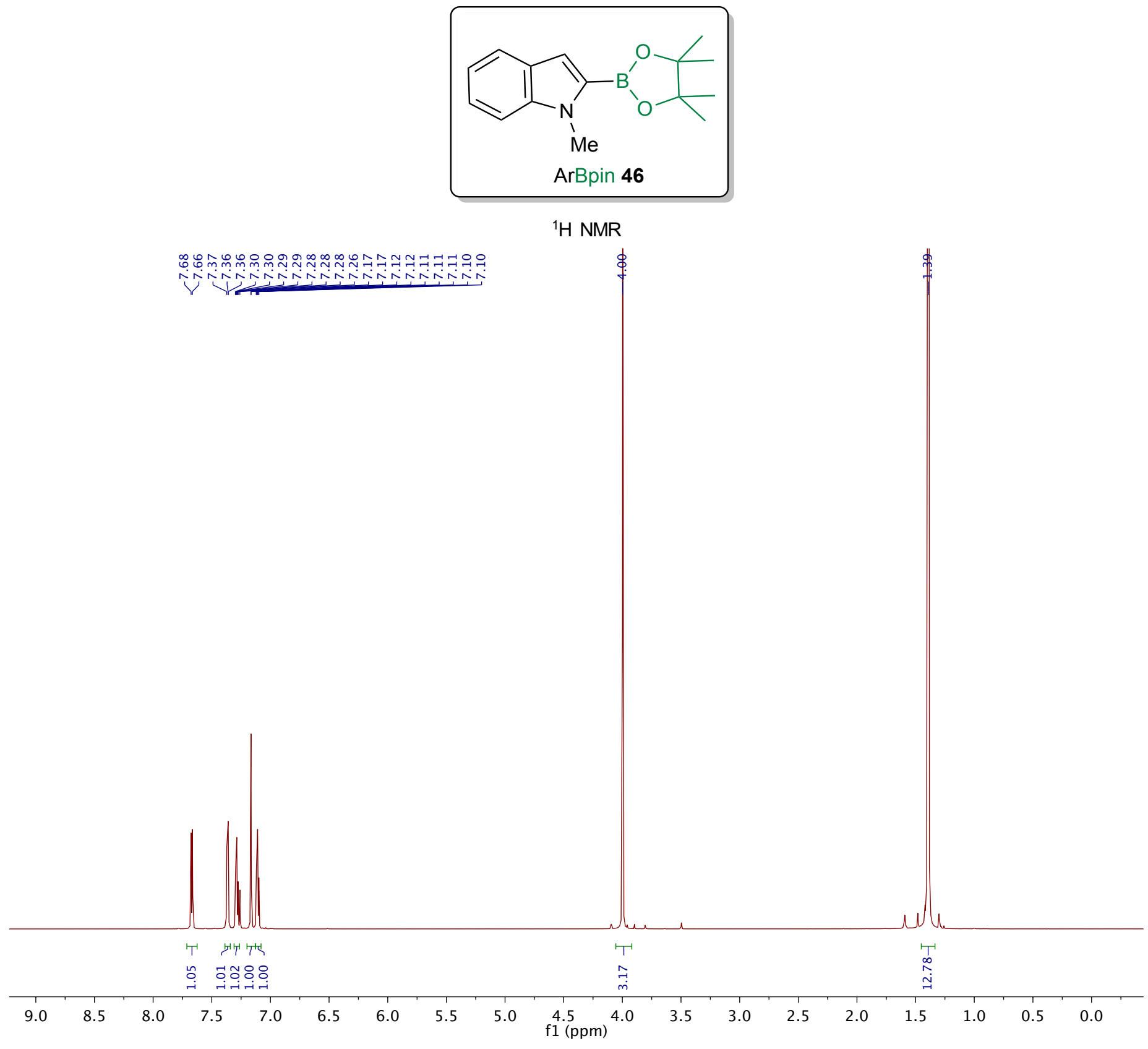

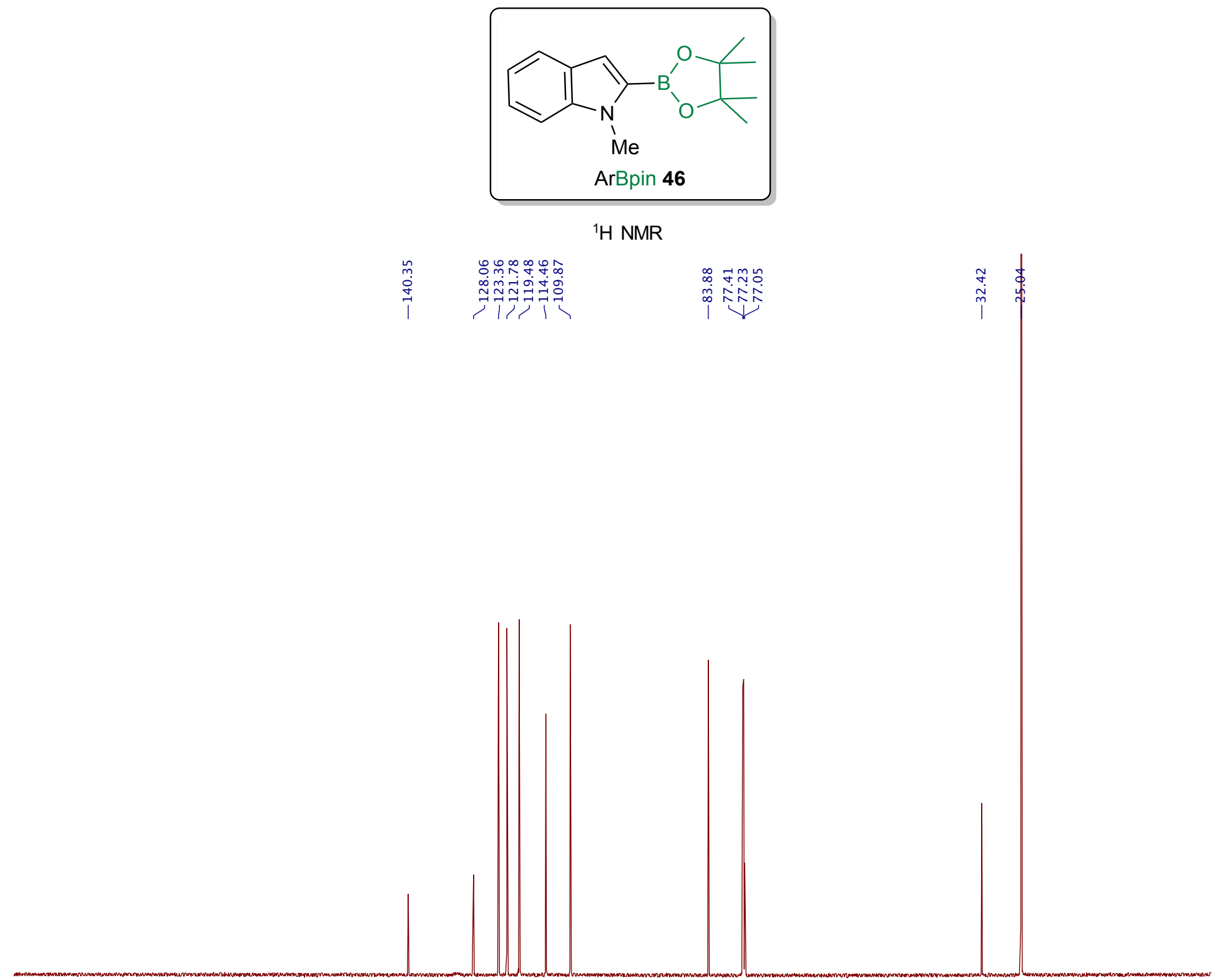


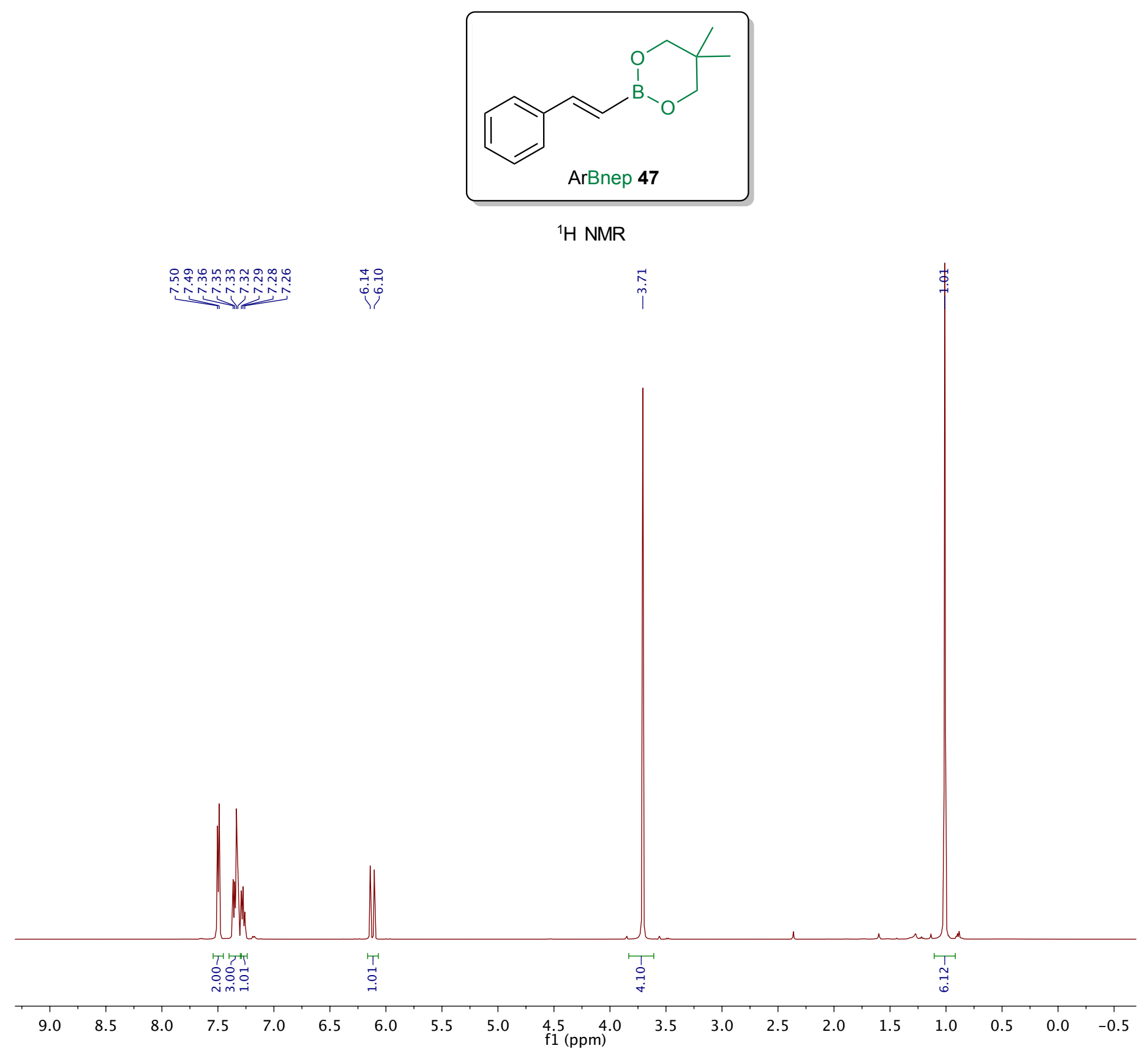




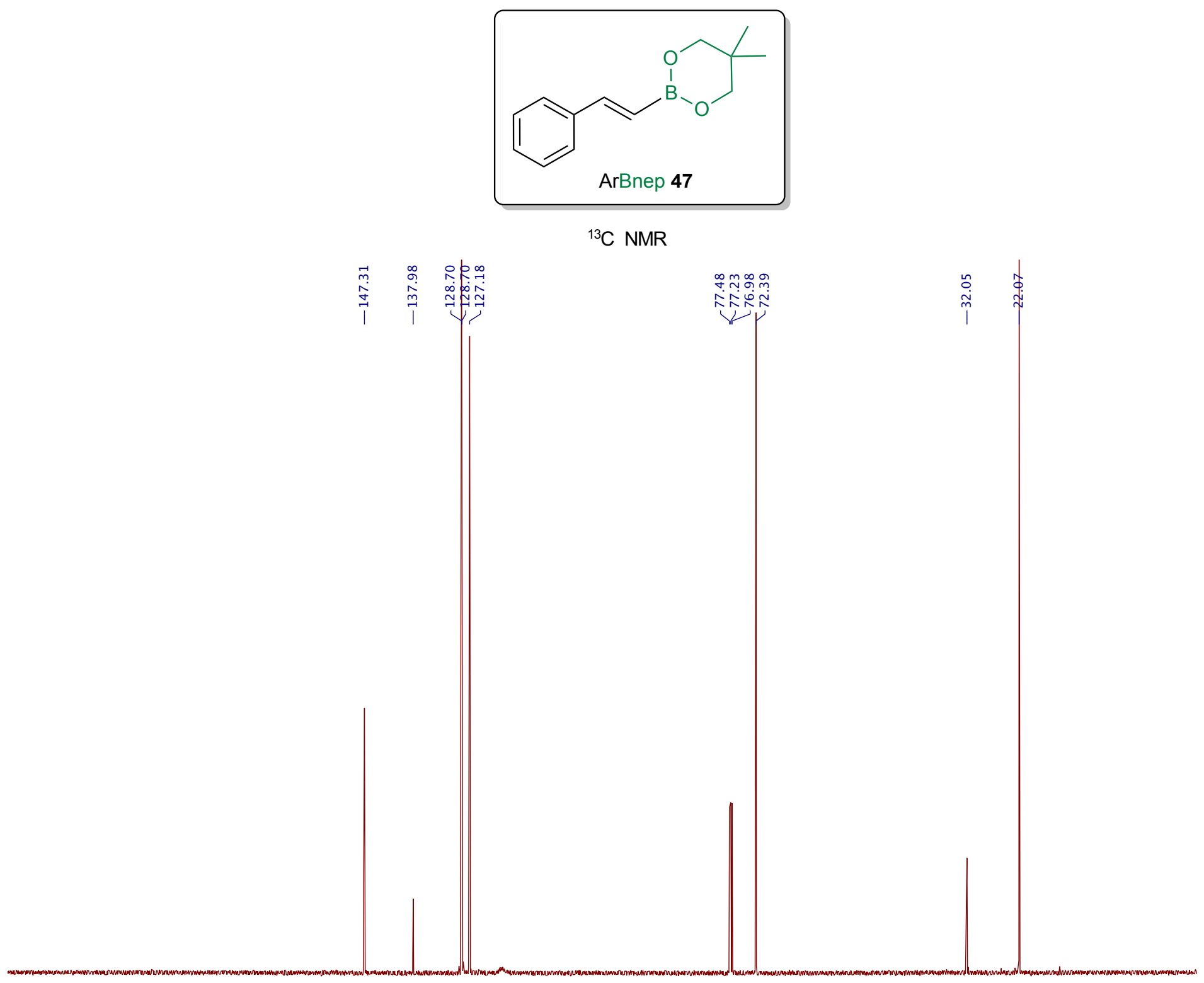

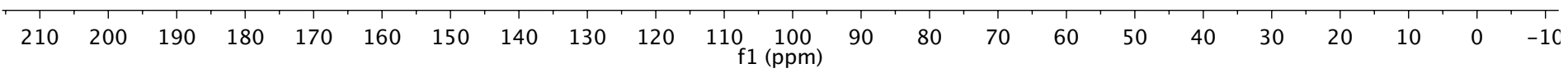




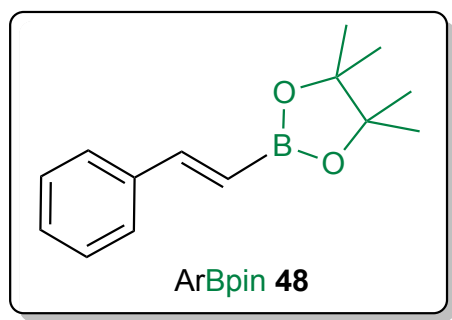

${ }^{1} \mathrm{H}$ NMR

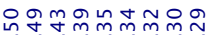

꾸ำำ

ำ

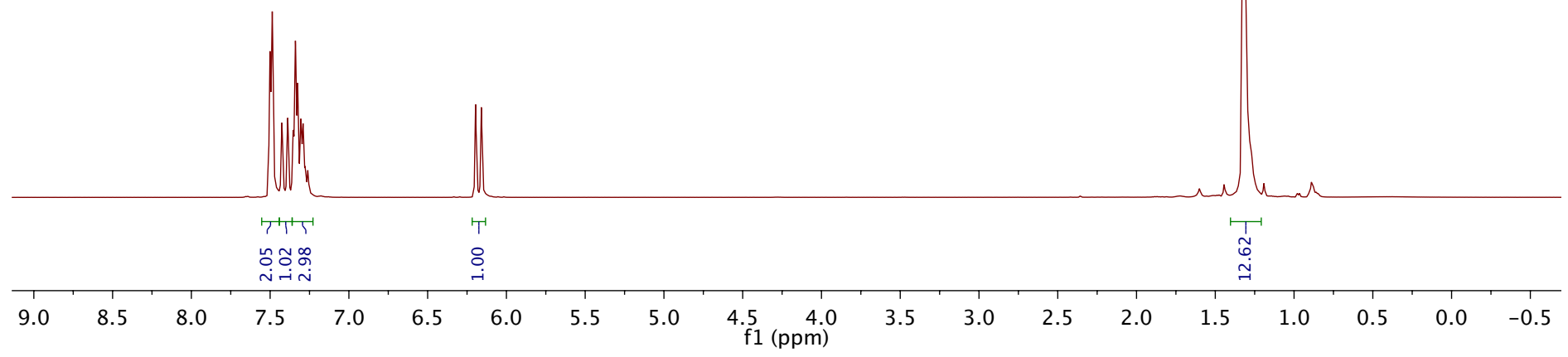




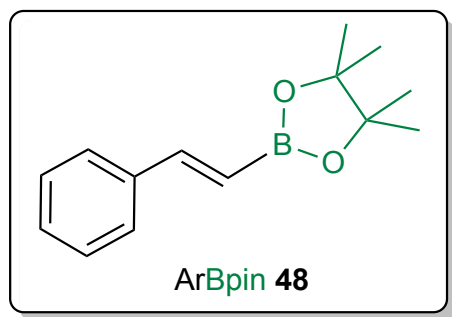

${ }^{13} \mathrm{C}$ NMR

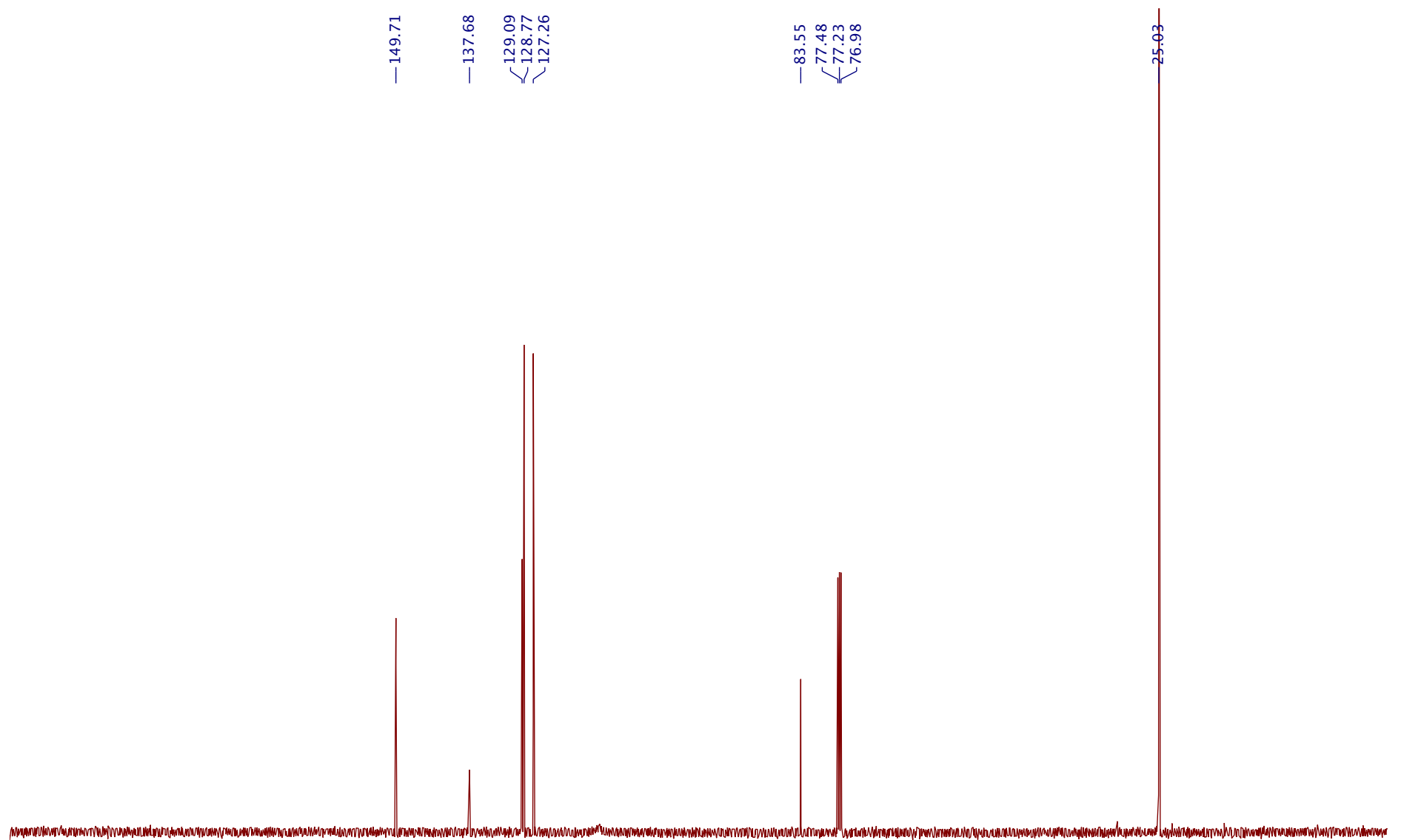

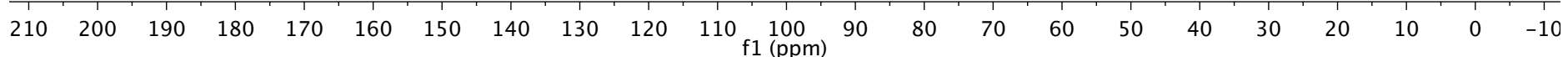




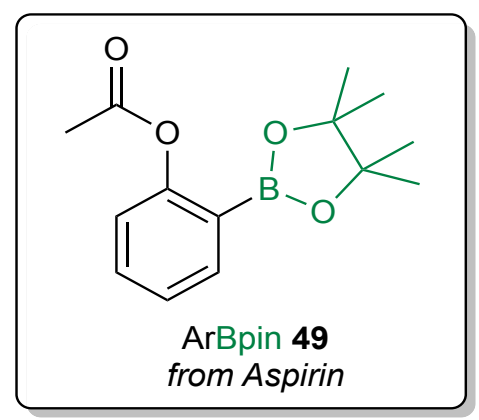

${ }^{1} \mathrm{H}$ NMR

$\infty_{\infty}$

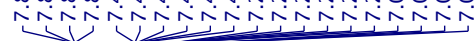
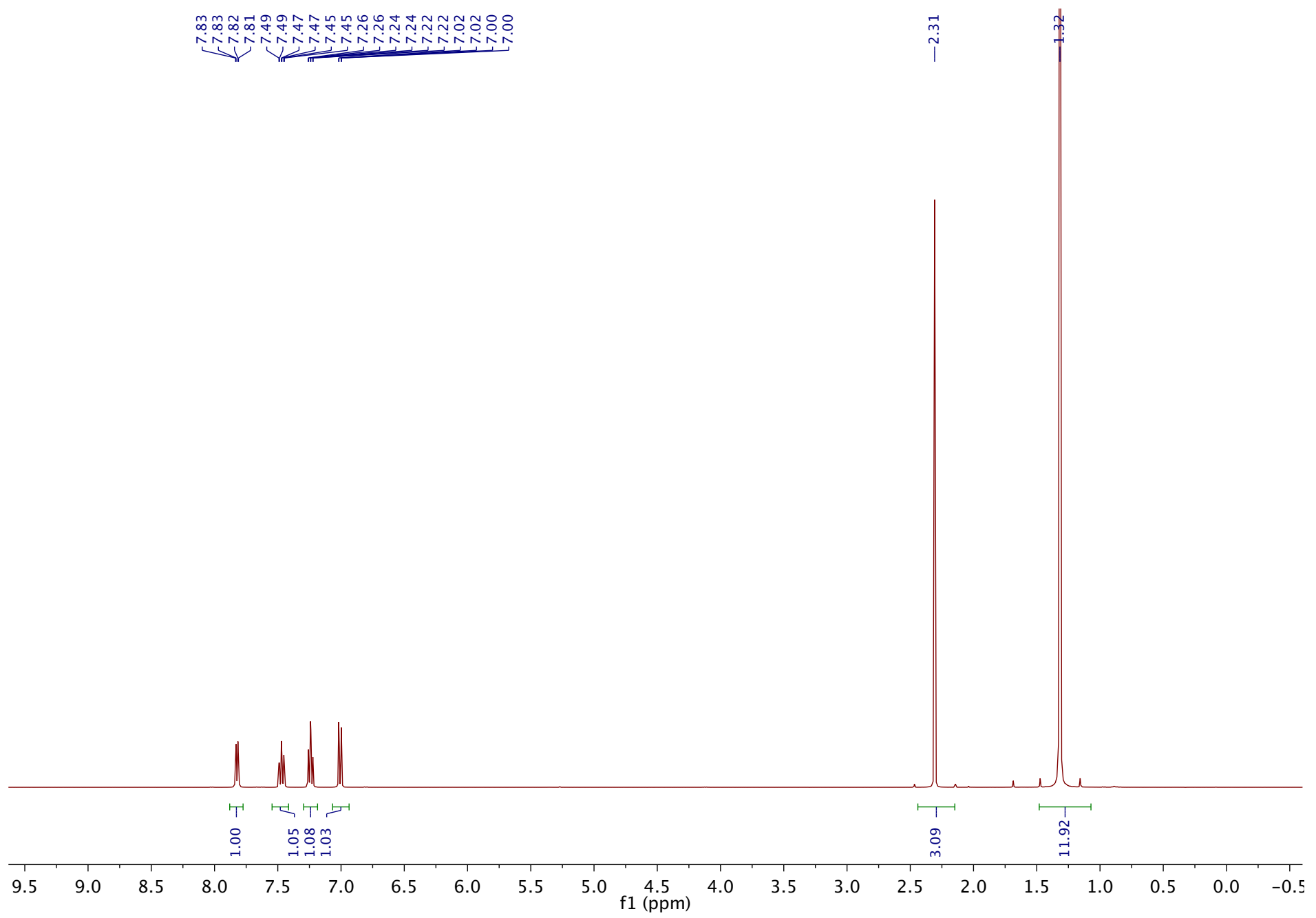


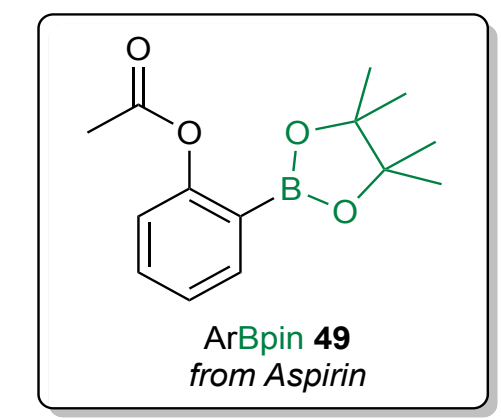

${ }^{13} \mathrm{C}$ NMR

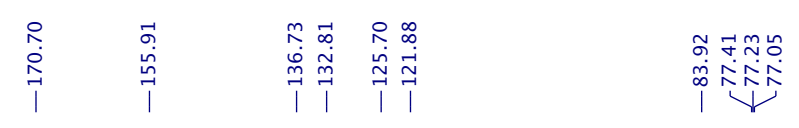

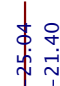

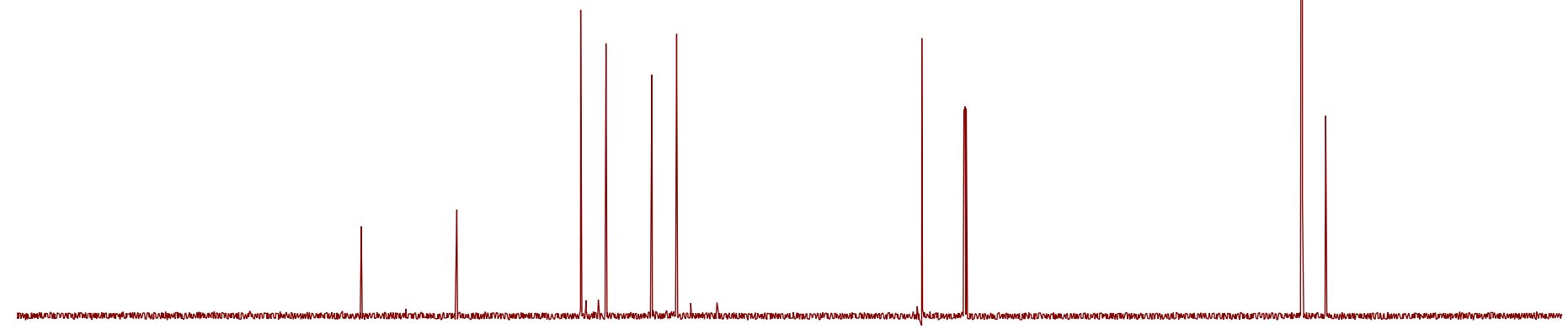




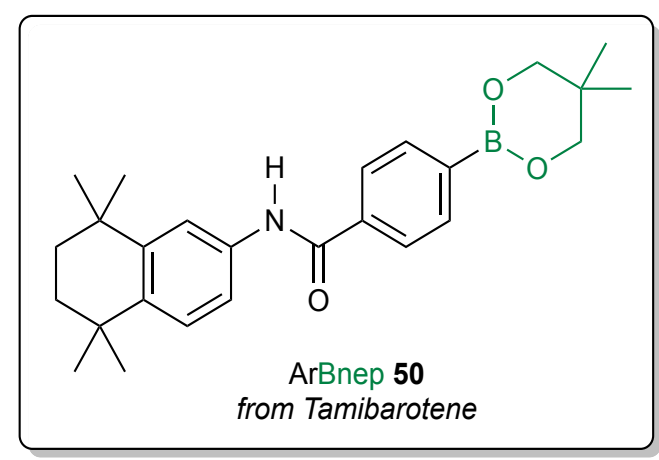

${ }^{13} \mathrm{C}$ NMR

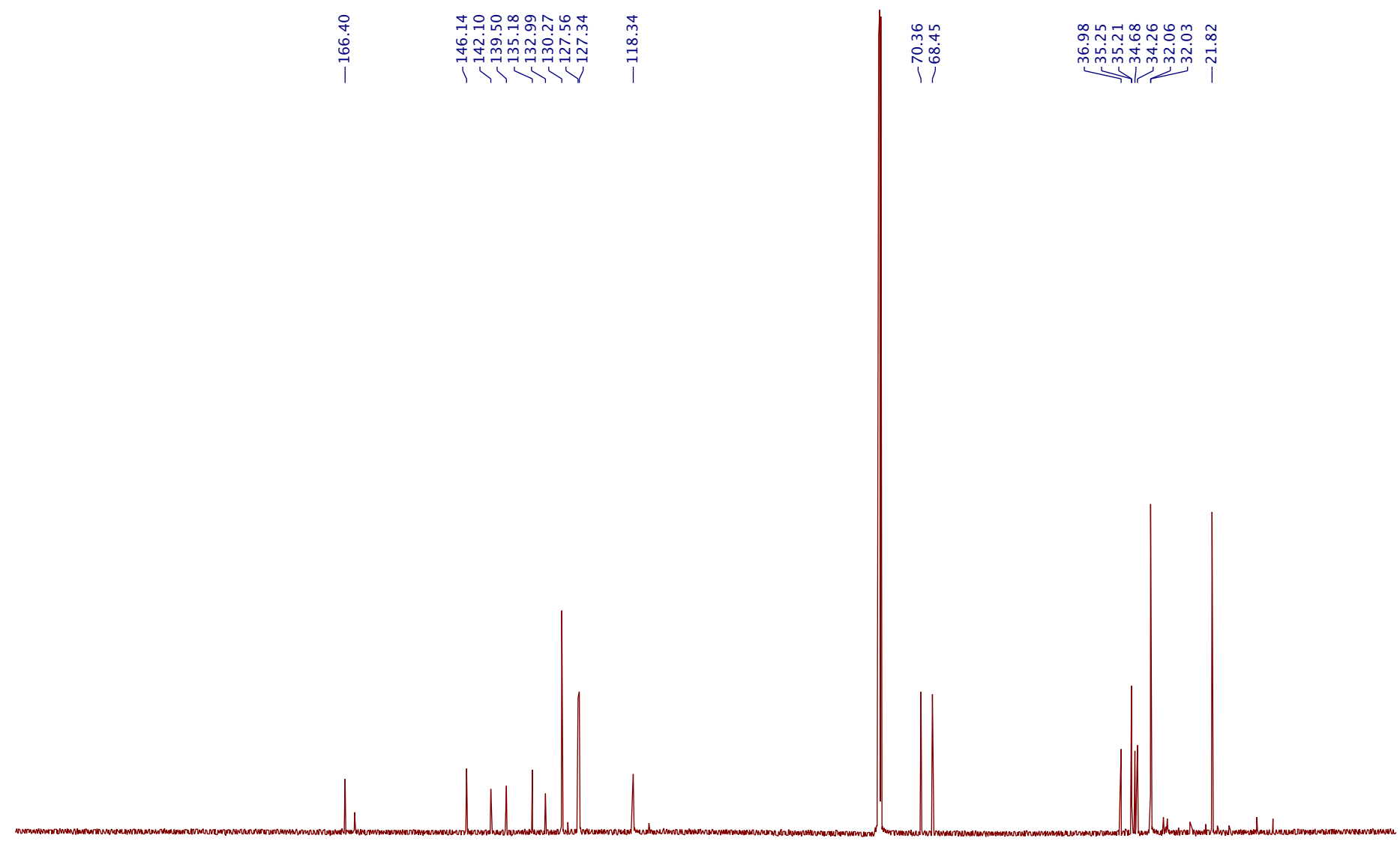

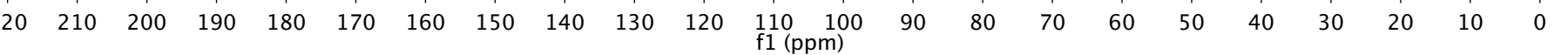




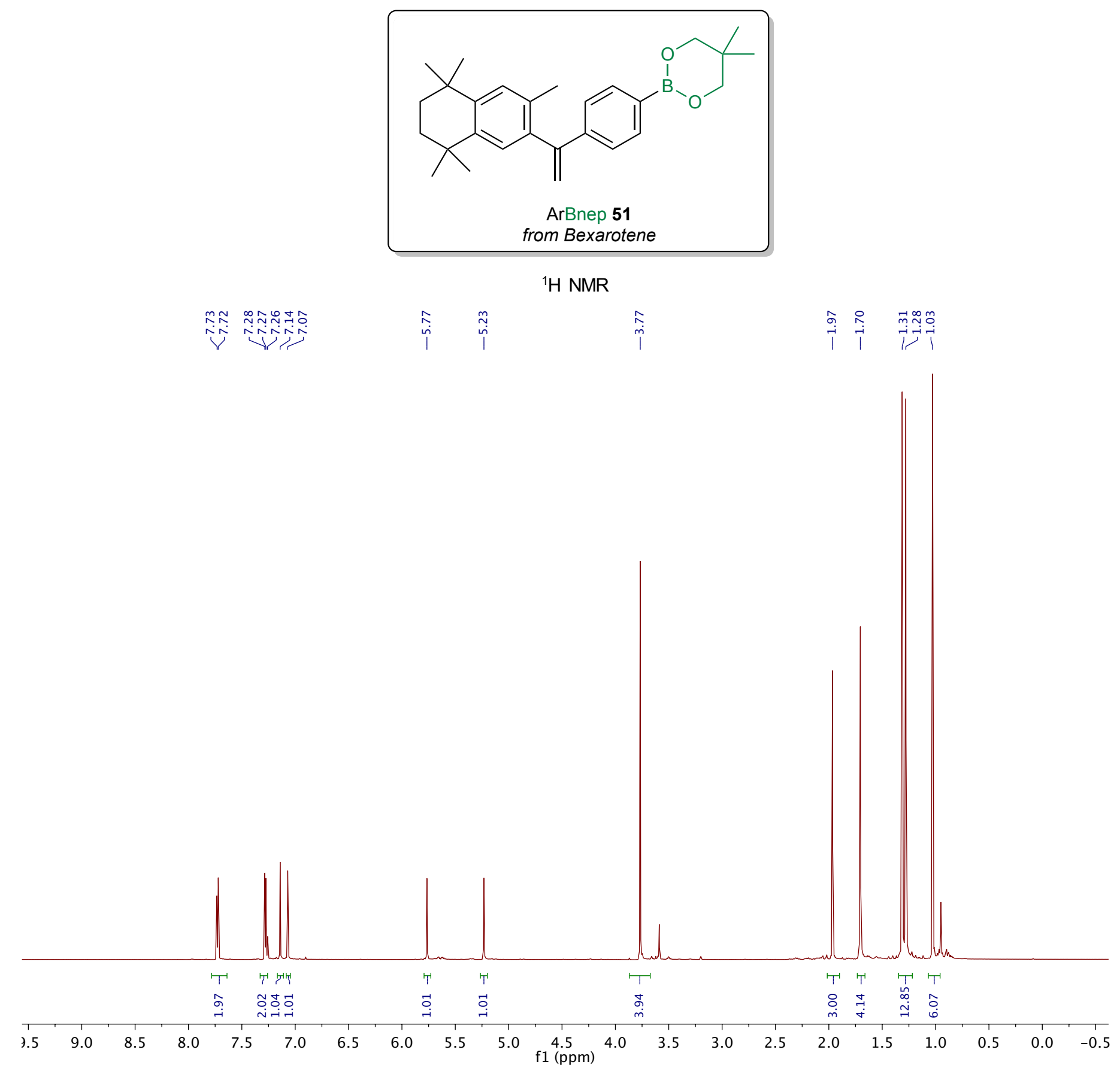



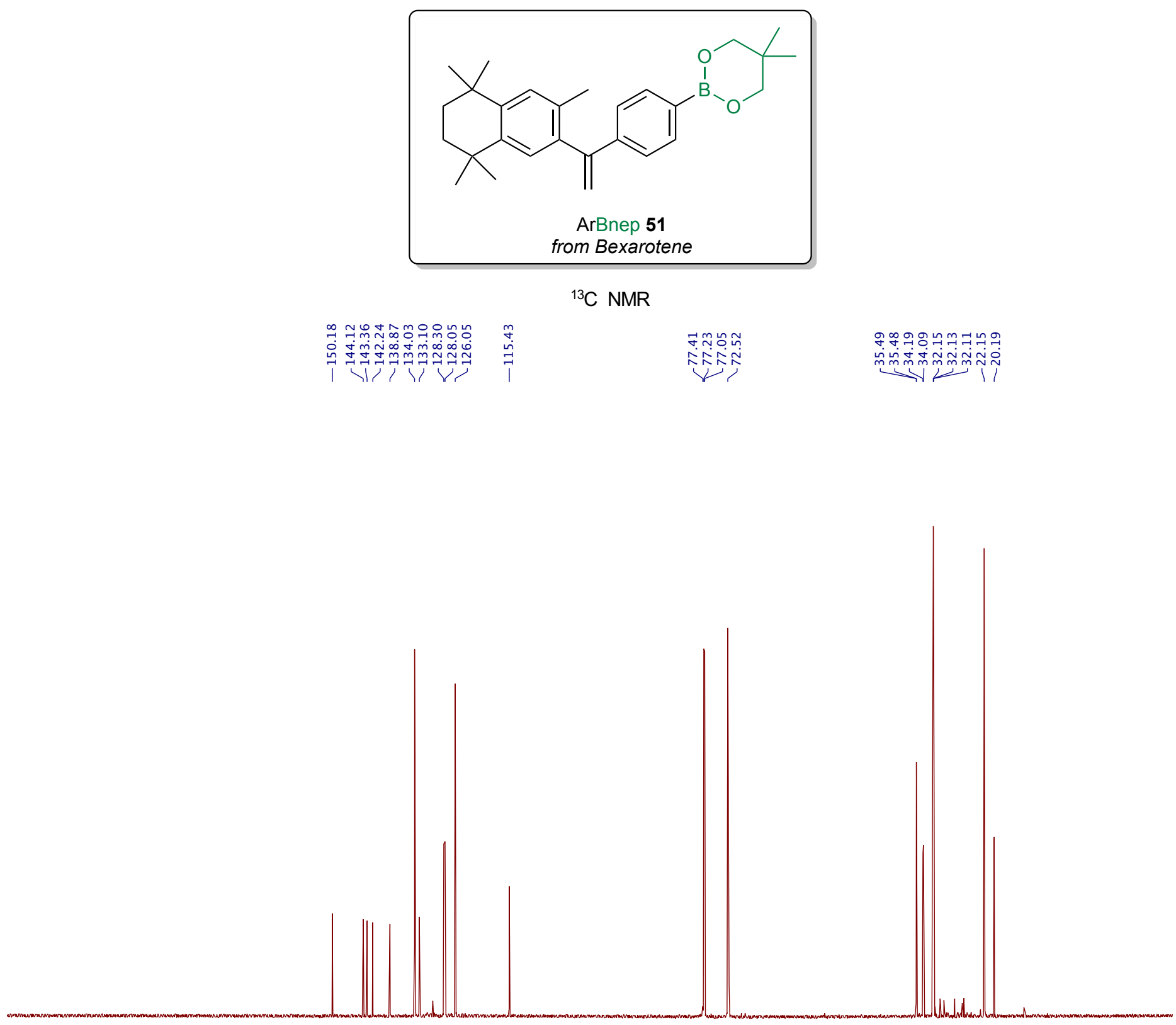


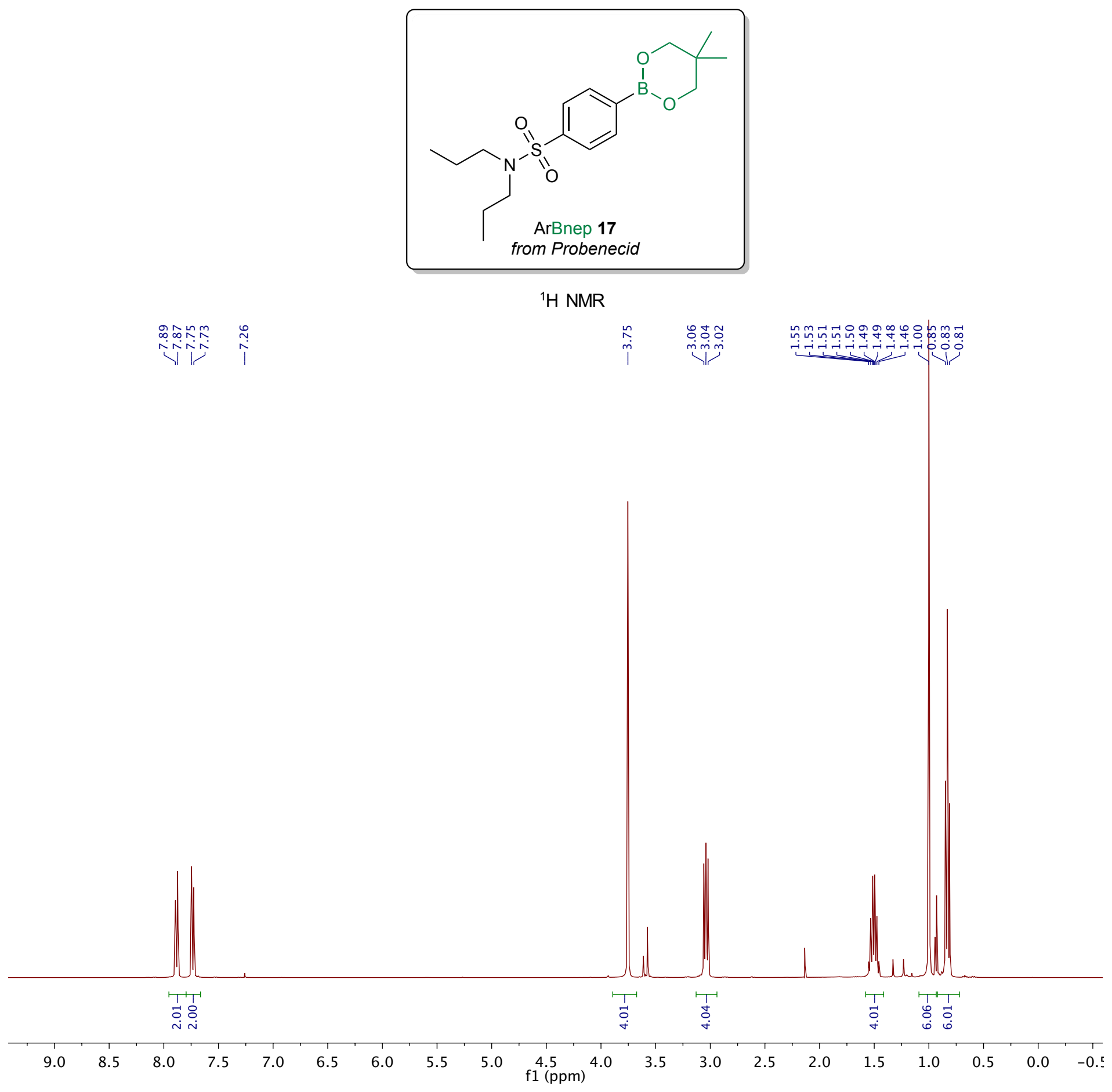




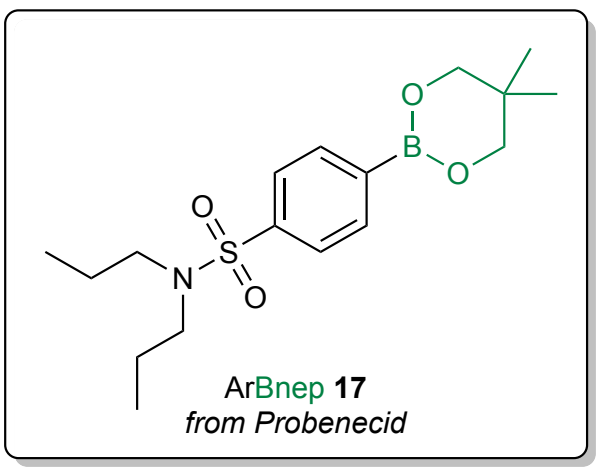

${ }^{13} \mathrm{C}$ NMR

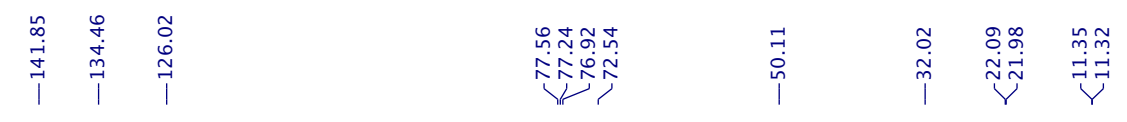

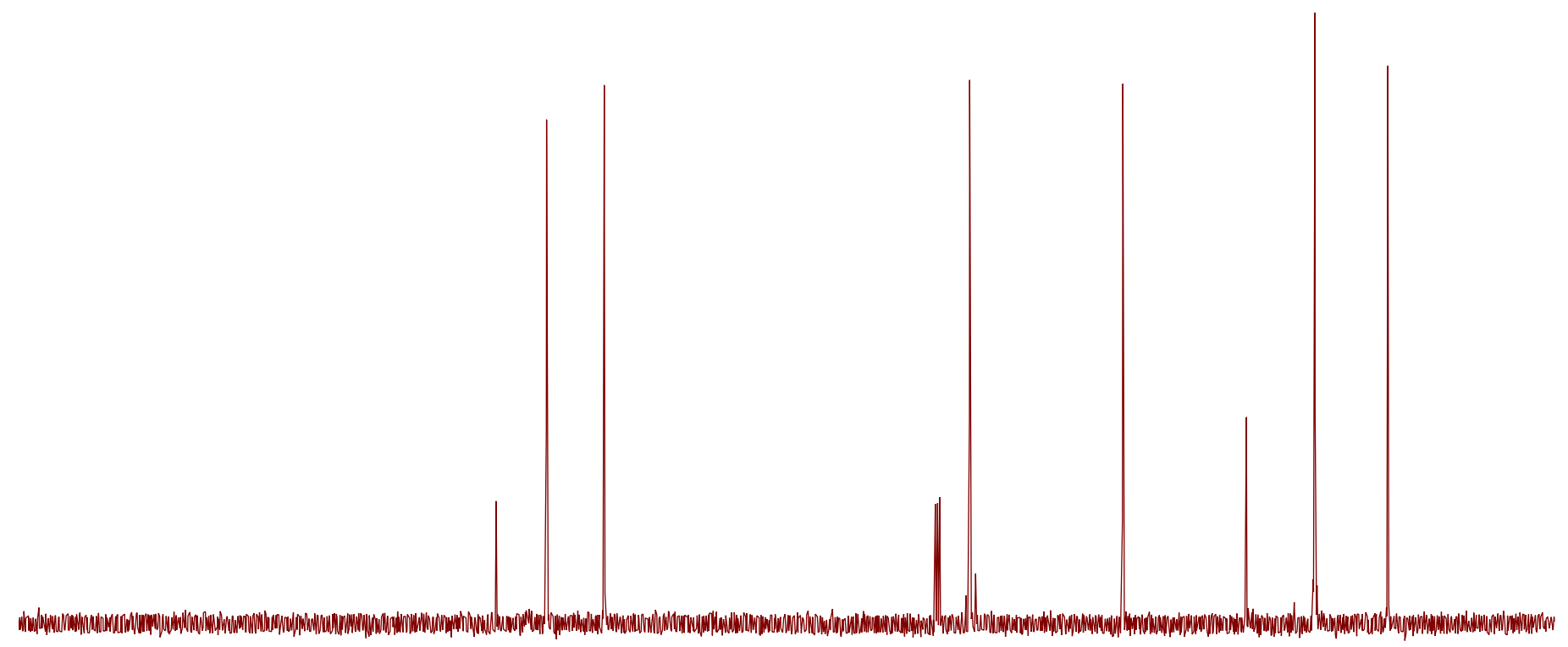

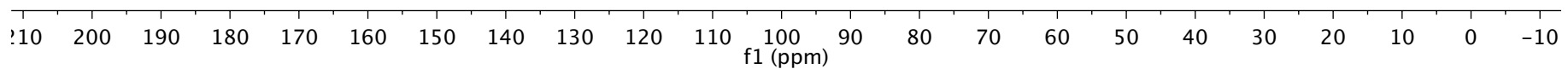




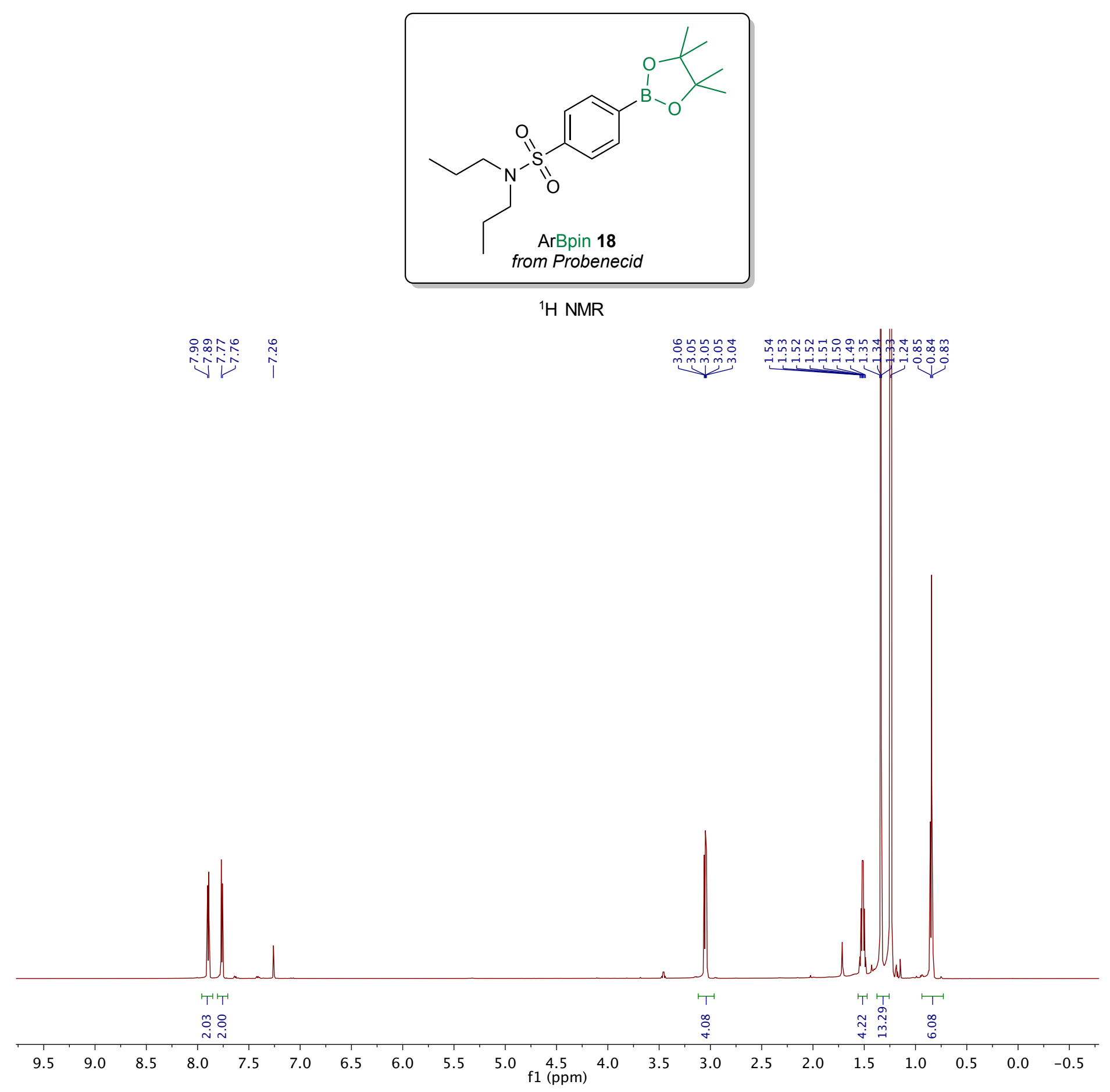




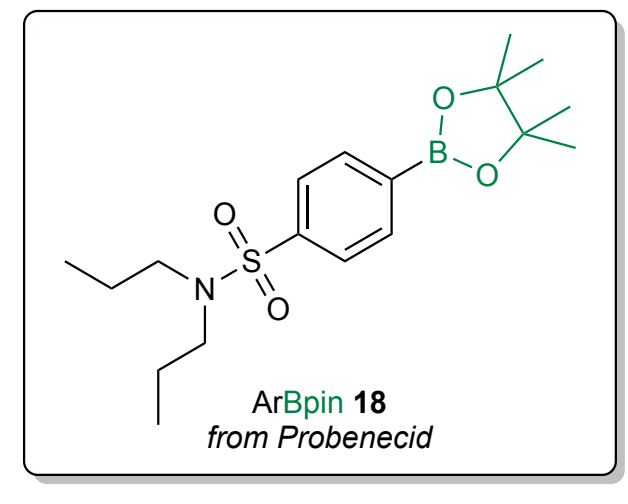

${ }^{13} \mathrm{C}$ NMR

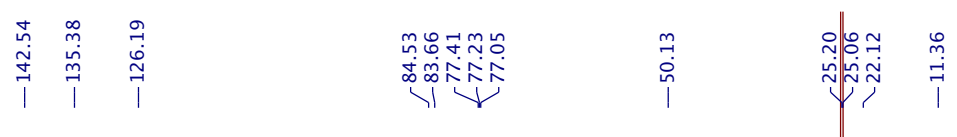

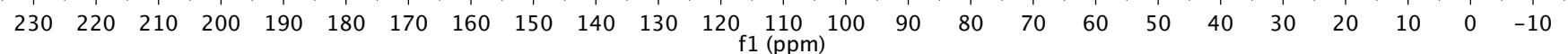




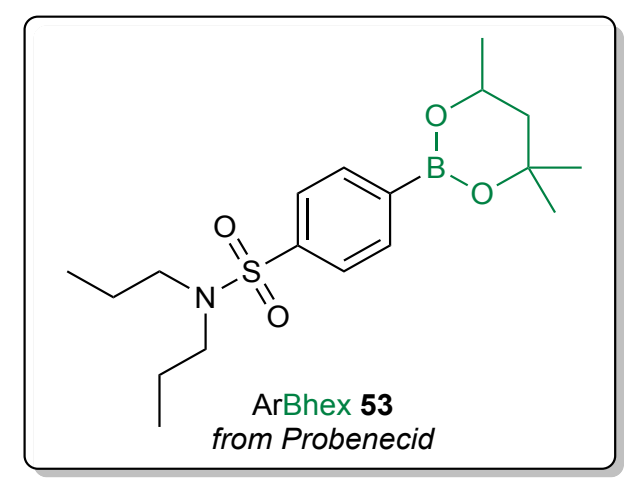

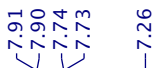

${ }^{1} \mathrm{H}$ NMR

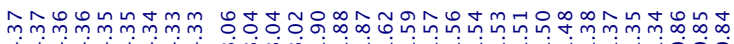

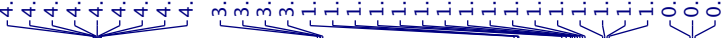

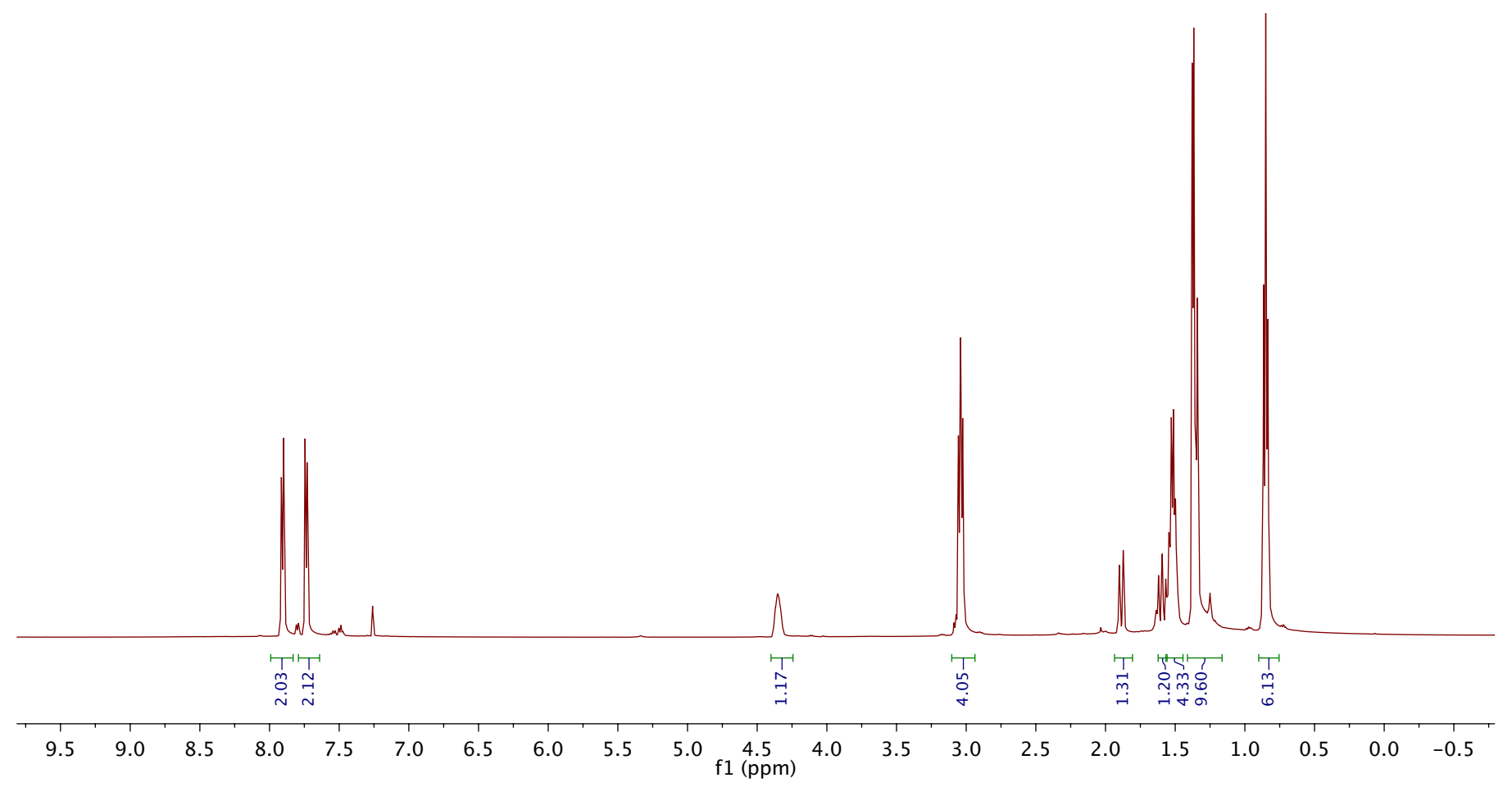



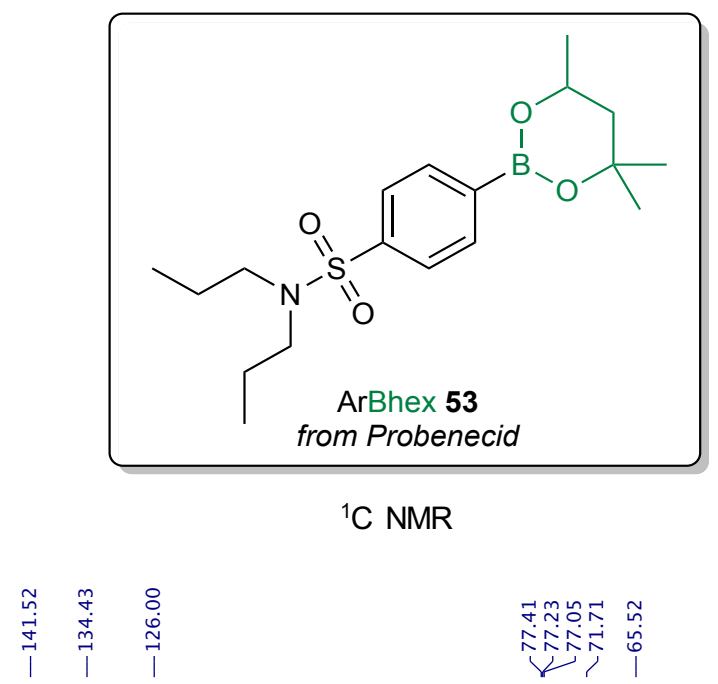

$\vec{\Phi}$

$\min 0$ 에 min 수

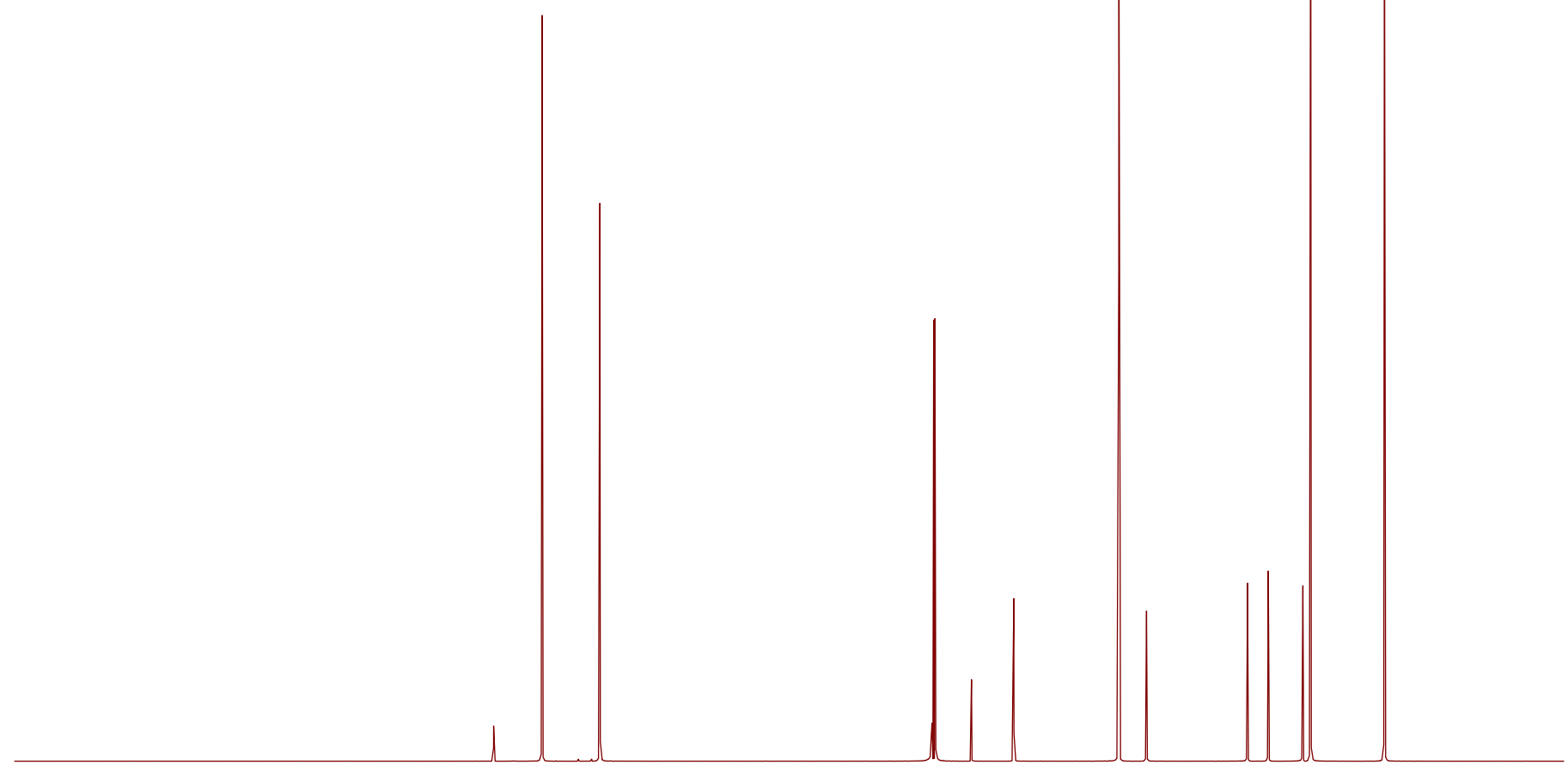

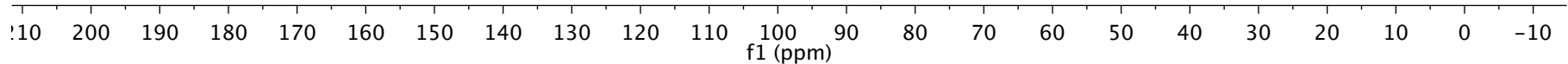




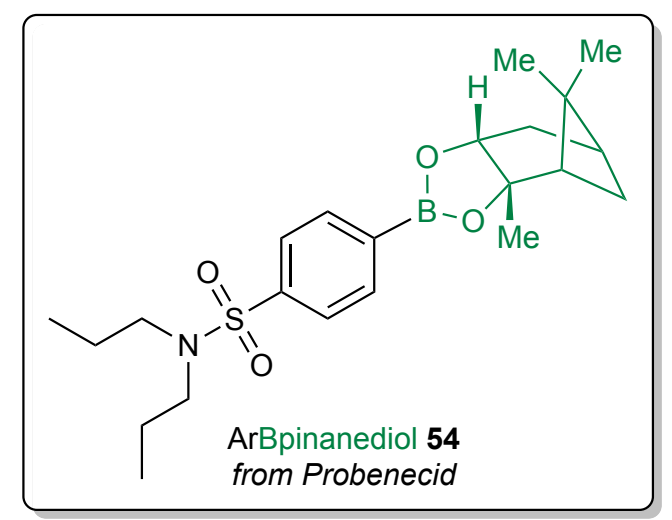

${ }^{1} \mathrm{H}$ NMR

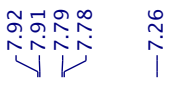

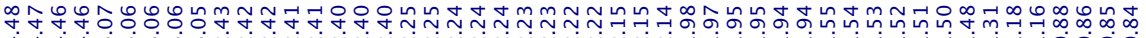

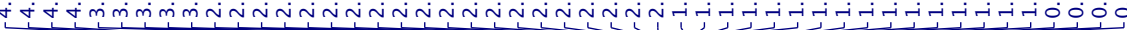

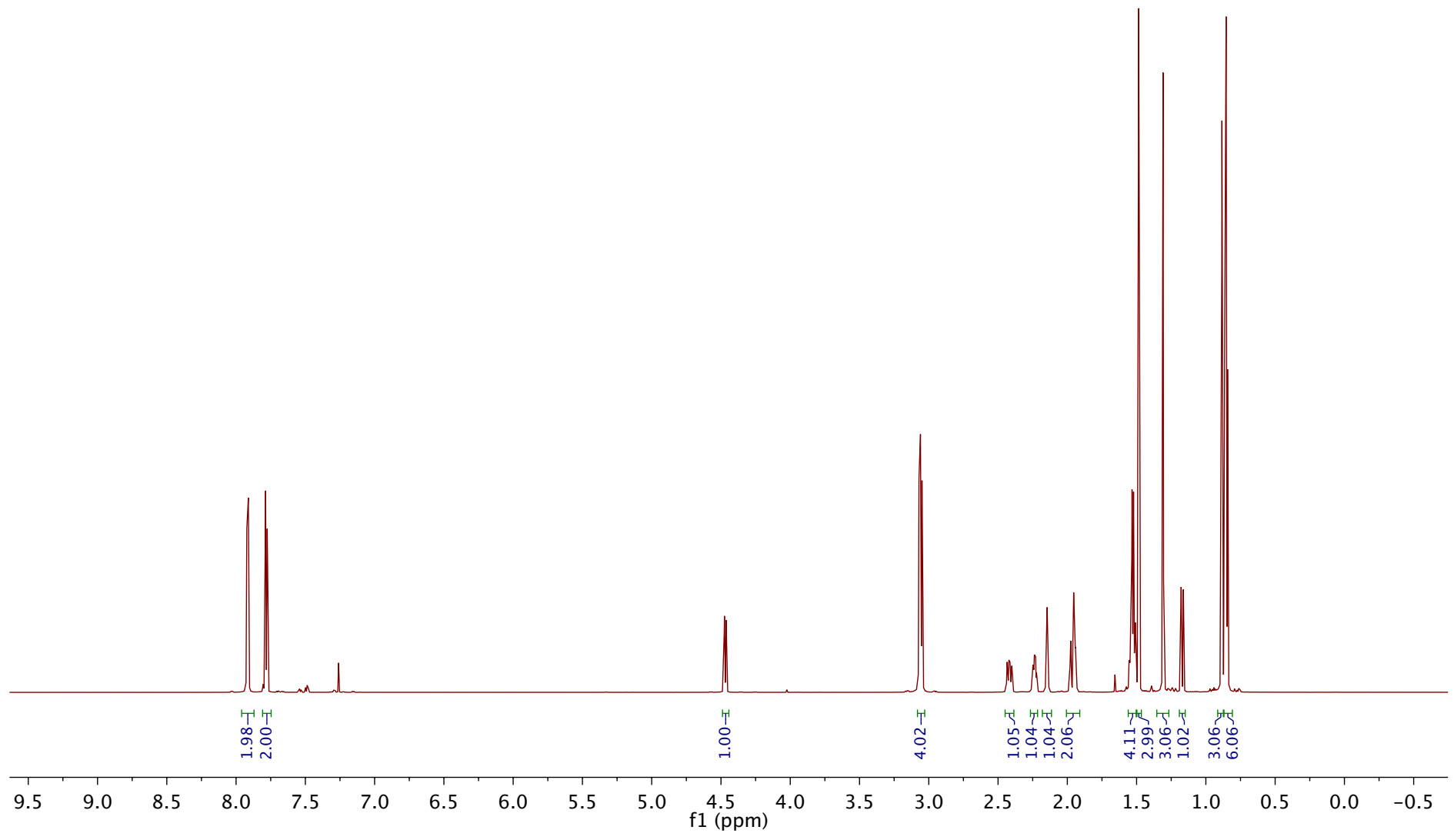




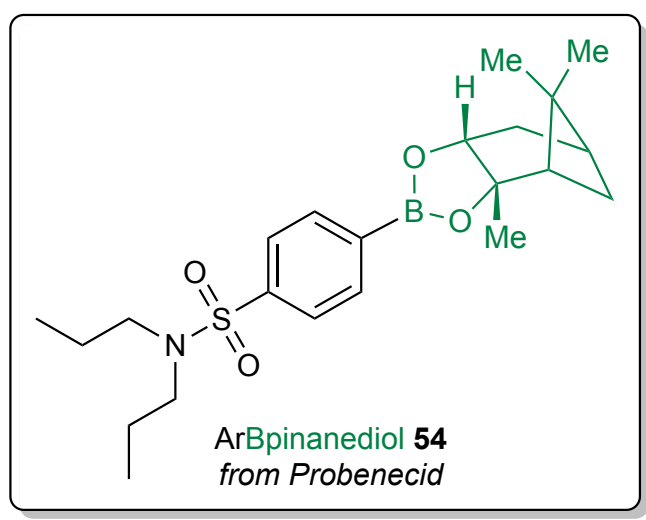

${ }^{13} \mathrm{C}$ NMR

\begin{tabular}{|c|c|c|c|}
\hline 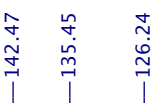 & 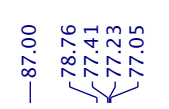 & 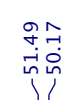 & 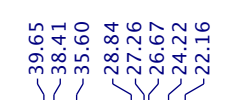 \\
\hline
\end{tabular}

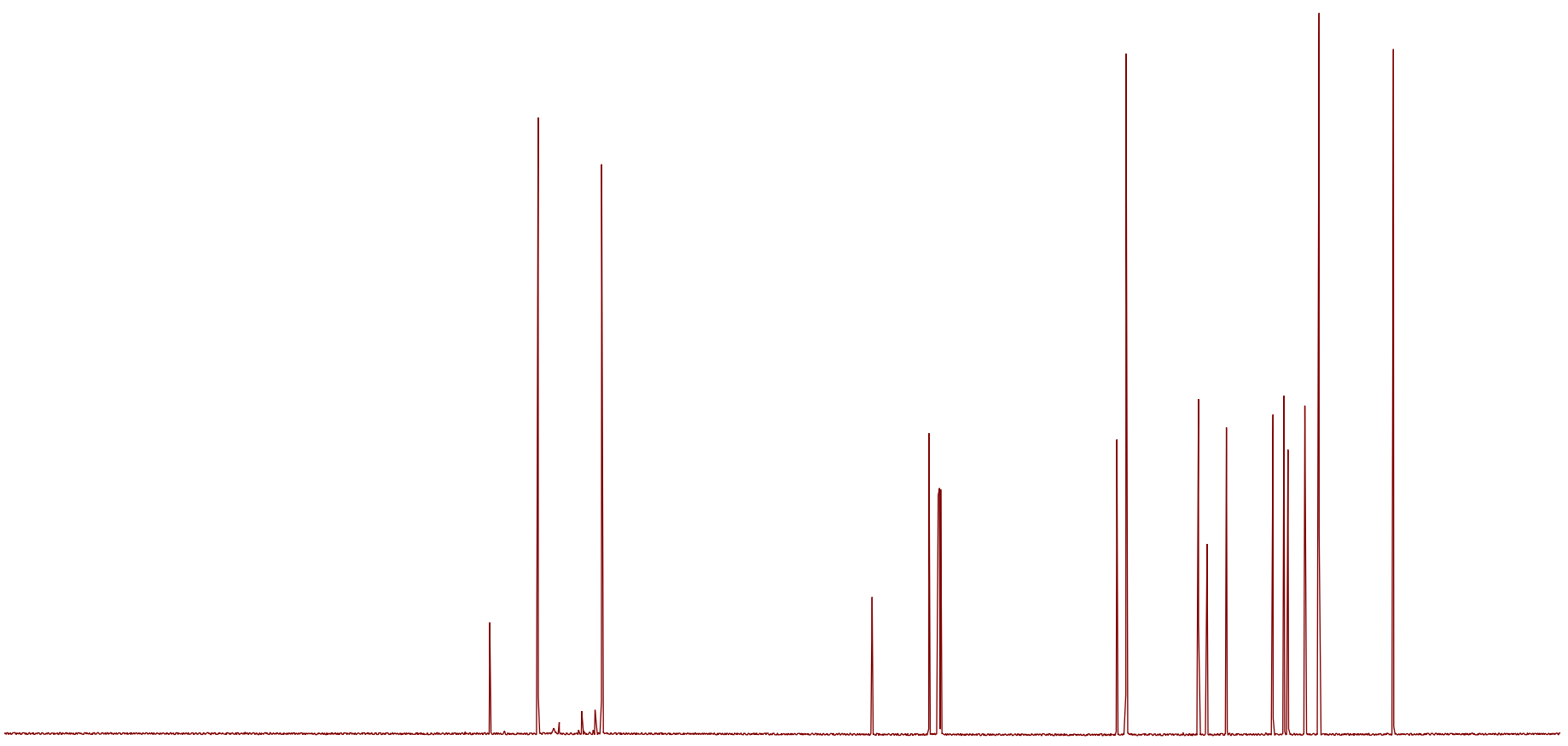




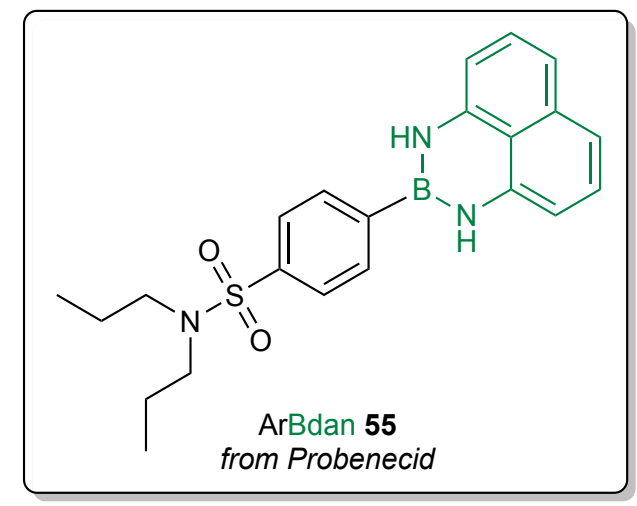

${ }^{1} \mathrm{H}$ NMR

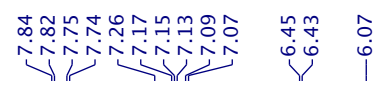

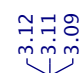

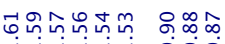

initin oij

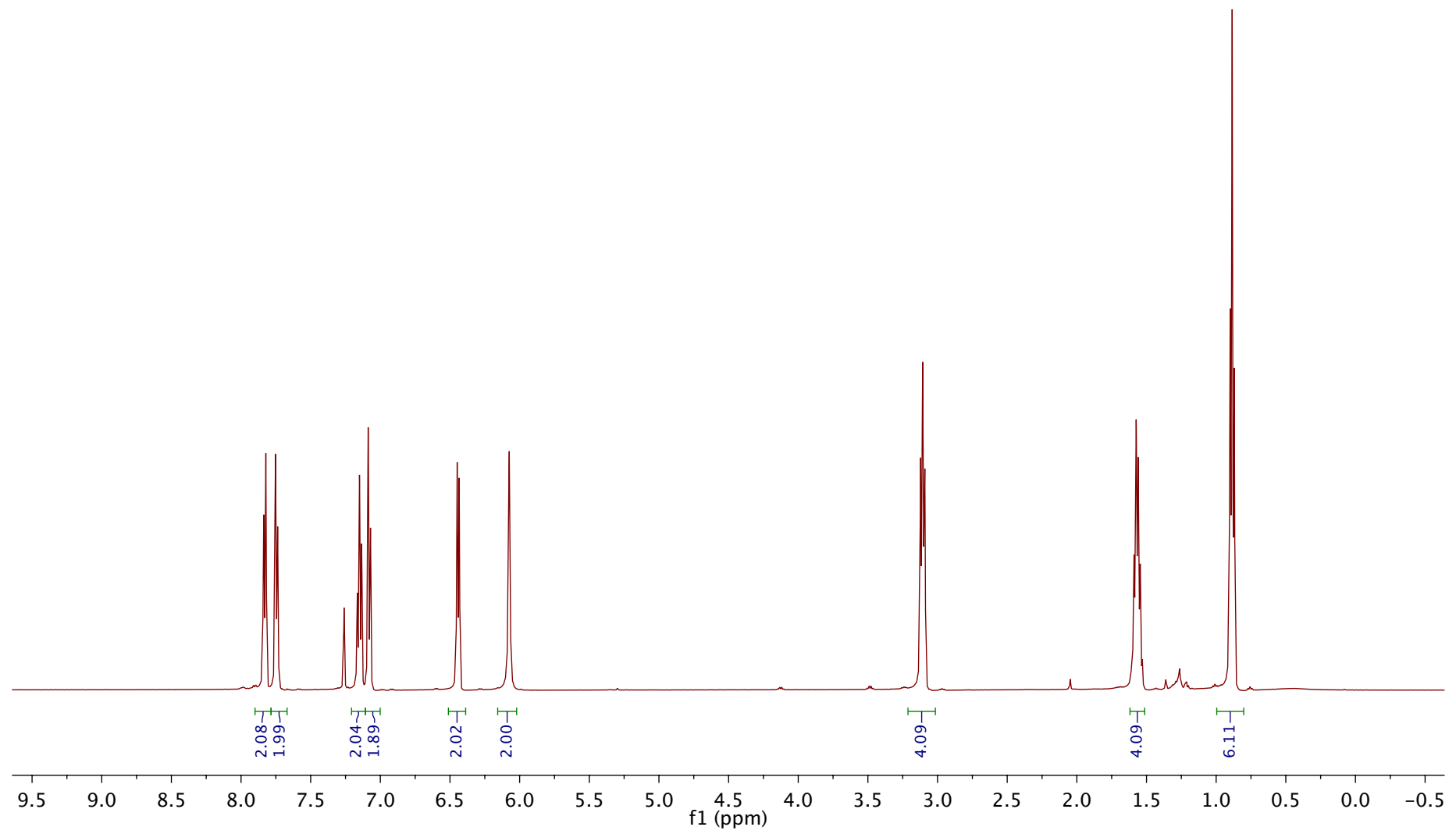




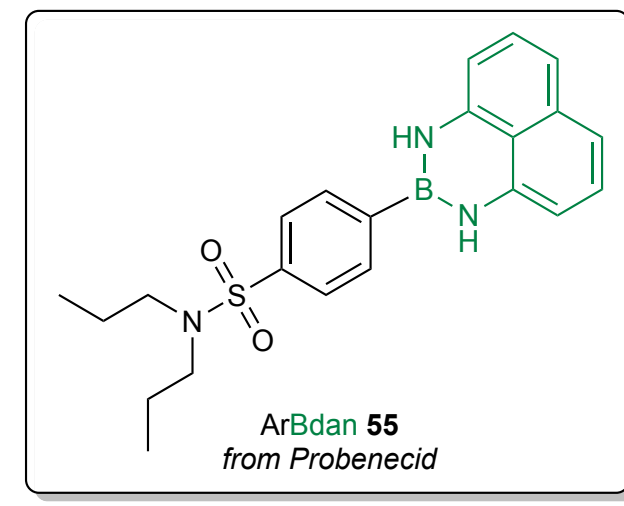

${ }^{13} \mathrm{C}$ NMR

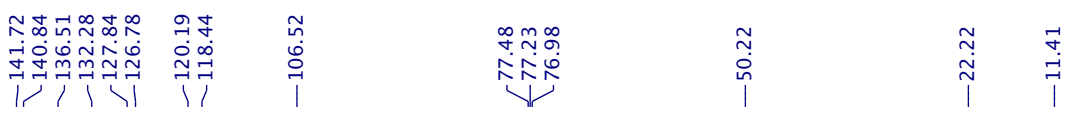

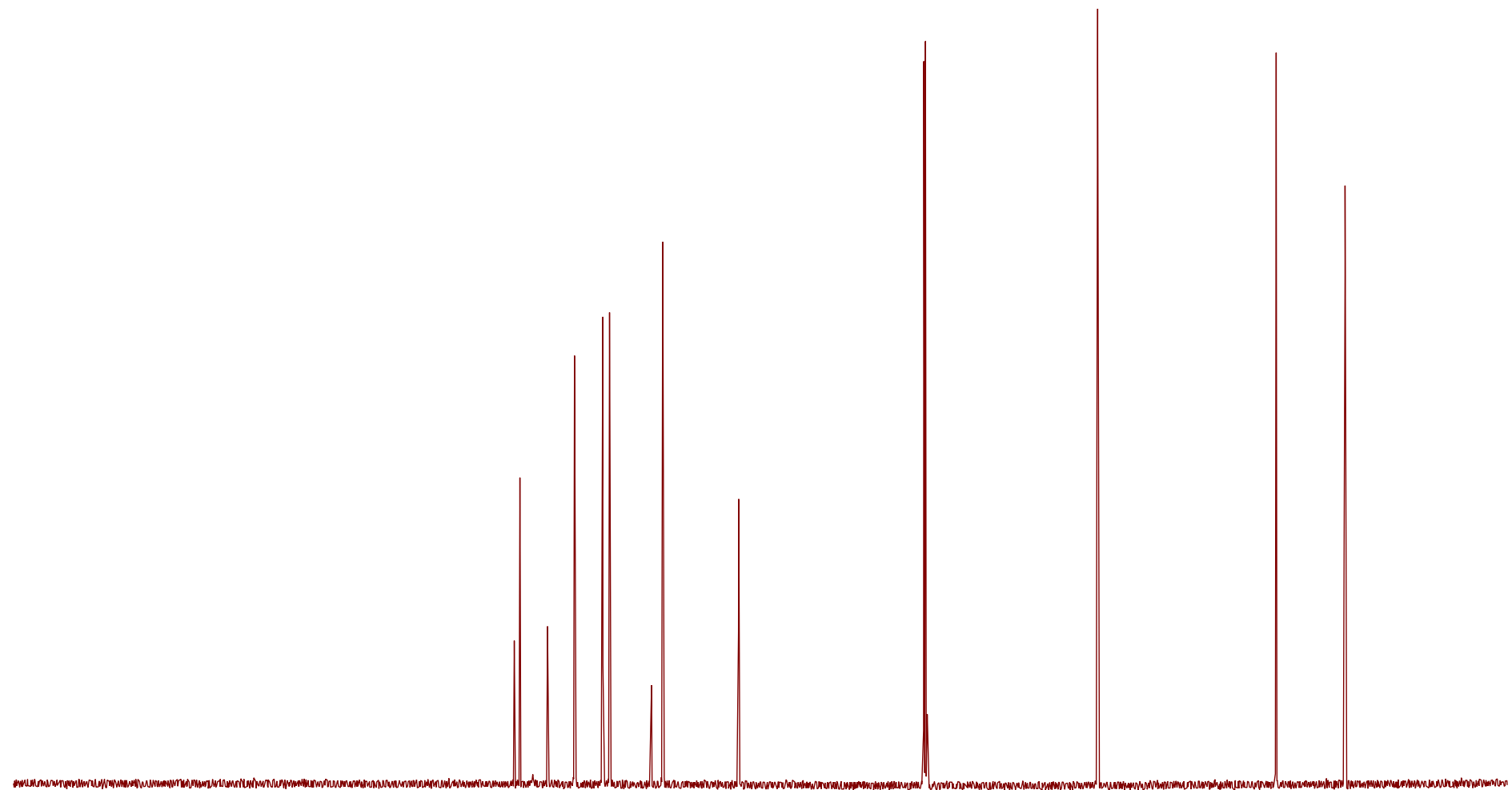

